archives-ouvertes

\title{
Dark Photon Search with the HPS Experiment at JLab Ani Simonyan
}

\section{To cite this version:}

Ani Simonyan. Dark Photon Search with the HPS Experiment at JLab. High Energy Physics Experiment [hep-ex]. Université Paris-Saclay, 2017. English. NNT : 2017SACLS561 . tel-01792977

\section{HAL Id: tel-01792977 https://tel.archives-ouvertes.fr/tel-01792977}

Submitted on 16 May 2018

HAL is a multi-disciplinary open access archive for the deposit and dissemination of scientific research documents, whether they are published or not. The documents may come from teaching and research institutions in France or abroad, or from public or private research centers.
L'archive ouverte pluridisciplinaire HAL, est destinée au dépôt et à la diffusion de documents scientifiques de niveau recherche, publiés ou non, émanant des établissements d'enseignement et de recherche français ou étrangers, des laboratoires publics ou privés. 


\section{Dark Photon Search with the HPS Experiment at JLab}

Thèse de doctorat de l'Université Paris-Saclay préparée à l'Universite Paris-Sud

École doctorale n576 PHENIICS: Particules, Hadrons, Énergie, Noyau, Instrumentation, Imagerie, Cosmos et Simulation Spécialité de doctorat : physique des particules

\section{Aní Símonyan}

Composition du Jury :

Elias Khan

Professeur, Université Paris-Sud,

Président du Jury

Institut de Physique Nucléaire Orsay

Stepan Stepanyan

Chercheur principal, Thomas Jefferson National Accelerator Facility

Rapporteur

Division de Physique

Achim Denig

Professeur, Johannes Gutenberg Université de Mainz,

Rapporteur

Institut de Physique Nucléaire

Eric Armengaud

Examinateur

Chercheur, Commissariat à l'énergie atomique et aux énergies alternatives,

Institut de recherche sur les lois fondamentales de l'Univers

Marco Battaglieri

Chercheur principal, Institut National de Physique Nucléaire

Section de Gênes

Michel Guidal

Directeur de recherche, Université Paris-Sud

Directeur de Thèse

Institut de Physique Nucléaire Orsay

\section{Raphael Dupre}

Invité

Charge de recherche, Université Paris-Sud

Institut de Physique Nucléaire Orsay 
To my family and to my indul-nephew for their unwavering support and encouragement over the years

en voor het zonnetje van mijn planeet wiens liefde mijn leven belicht. 


\section{Contents}

$\begin{array}{ll}\text { Introduction } & 1\end{array}$

1. Physics Motivation 3

1.1. Introduction . . . . . . . . . . . . . . . . 3

1.2. Matter in Cosmology . . . . . . . . . . . . . . . 4

1.2.1. Rotational Curves of Galaxies . . . . . . . . . . . . . 6

1.2.2. Gravitational Lensing and Bullet Cluster . . . . . . . . . . . . 6

1.2.3. Bullet Cluster Observation . . . . . . . . . . . . . . 7

1.2.4. Evolving Universe in the Big Bang Model . . . . . . . . . . . 99 9

1.2.5. WIMP miracle . . . . . . . . . . . . . . . . . 10

1.2.6. The Quantity of Matter in the Universe . . . . . . . . . . . 12

1.3. Matter in Particle Physics . . . . . . . . . . . . . . . . . . 13

1.3.1. Problems of the Standard Model and Proposed Theoretical

Solutions . . . . . . . . . . . . . . . . 14

1.4. Hidden Sector . . . . . . . . . . . . . . . . . . 18

1.4.1. Portals to The Hidden World . . . . . . . . . . . . . . . . 19

1.4.2. Kinetic Mixing For Vector Portal . . . . . . . . . . . . . . . . 20

1.4.3. Anomalous Magnetic Moment . . . . . . . . . . . . . 22

1.4.4. Motivation for Dark Photon Searches with an $e^{-}$Beam . . . . 24

1.4.5. The Experimental Considerations for Dark Photon Search in

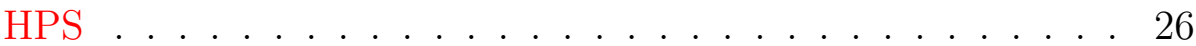

1.5. Conclusion . . . . . . . . . . . . . . . . . . . . 29

2. Dark Photon Hunt in the World 31

2.1. Introduction . . . . . . . . . . . . . . . . . . . 31

2.2. Beam-Dump Experiments . . . . . . . . . . . . . . 31

2.3. Collider Experiments . . . . . . . . . . . . . . . . . 34

2.4. Fixed Target Experiments . . . . . . . . . . . . . . . . 36

2.5. Searches in Dark Photon Invisible Decays . . . . . . . . . . . . . . . . 38

2.6. Higgs portal searches . . . . . . . . . . . . . . . . . . . . . . . . . . . . . . . . . . . . . . .

2.7. Conclusion . . . . . . . . . . . . . . . . . . . 42

3. Detector Setup and Performance $\quad \mathbf{4 5}$

3.1. Introduction . . . . . . . . . . . . . . . . . . . 45

3.2. The CEBAF Accelerator . . . . . . . . . . . . . . 45

3.3. The HPS Detector . . . . . . . . . . . . . . . . 47

3.3.1. The Beamline . . . . . . . . . . . . . . . . 48 
3.3.2. The Beam . . . . . . . . . . . . . . . . . 49

3.3.3. The Target . . . . . . . . . . . . . . . 50

3.4. The Tracker: Silicon Vertex Tracker . . . . . . . . . . . . . . . 52

3.5. The Electromagnetic Calorimeter . . . . . . . . . . . . . . 53

3.5.1. ECal Design . . . . . . . . . . . . . . . 53

3.5.2. Signal Processing in the ECal . . . . . . . . . . . . . 54

3.5.3. JLab FADC250 in ECal . . . . . . . . . . . . . . . . 56

3.6. Trigger and Data Acquisition . . . . . . . . . . . . . . . . 56

3.6.1. The Trigger System . . . . . . . . . . . . . . . 57

3.6.2. SVT Data Acquisition . . . . . . . . . . . . . . . 59

3.6.3. ECal Readout with Flash ADC250 . . . . . . . . . . . 60

3.6.4. HPS Triggers . . . . . . . . . . . . . . . . . . . . . . . . . . 61

3.7. Conclusion . . . . . . . . . . . . . . . . . 62

4. Detector Simulation and Reconstruction $\quad \mathbf{6 3}$

4.1. Introduction . . . . . . . . . . . . . . . . . 63

4.2. The Ecal Geometry Simulation . . . . . . . . . . . . . . . . 64

4.2.1. ECal Geometry Correction . . . . . . . . . . . . 65

4.3. Event Reconstruction . . . . . . . . . . . . . . . . . 67

4.3.1. SVT Hit Reconstruction . . . . . . . . . . . . . . . . . . 68

4.3.2. Track Reconstruction . . . . . . . . . . . . . . . . 68

4.3.3. ECal Hit Reconstruction . . . . . . . . . . . . . . . . . . . . 69

4.3.4. Track-Cluster Matching Algorithm . . . . . . . . . . . . 70

4.4. ECal Corrections . . . . . . . . . . . . . . . . . . . 72

4.4.1. Evaluation of the Energy Correction Function . . . . . . . . 72

4.5. Calibration of the ECal . . . . . . . . . . . . . . 73

4.5.1. Calibration with cosmic rays . . . . . . . . . . . . . . 74

4.5.2. Energy Calibration with Beam Data . . . . . . . . . . 78

4.5.3. Studying the ECal Time Resolution with the Beam Data . . . 79

4.6. Conclusion . . . . . . . . . . . . . . . . . 81

5. Background Simulations $\quad \mathbf{8 5}$

5.1. Introduction . . . . . . . . . . . . . . . . 85

5.2. WAB and Multiple Coulomb Scattering Backgrounds . . . . . . . . . 85

5.3. Prompt Single Vertex QED Background . . . . . . . . . . . . . . 86

5.3.1. Radiative and Bethe-Heitler Trident Simulations . . . . . . . . 87

5.3.2. MadGraph5 Implementation . . . . . . . . . . . . . . . . . . . 87

5.3.3. The Theoretical Model for Monte Carlo Calculations . . . . . 87

5.3.4. The Monte Carlo Simulation . . . . . . . . . . . . . . . . . 88

5.4. Comparison . . . . . . . . . . . . . . . . . 90

5.5. Conclusion . . . . . . . . . . . . . . . . . . 92

6. Data Analysis: Moller electrons 101

6.1. The Moller Scattering . . . . . . . . . . . . . . . . . . . 101 
6.2. Moller Analysis . . . . . . . . . . . . . . . . . . . . . . . . 102

6.2.1. Data Sample . . . . . . . . . . . . . . . . . . . 102

6.2.2. Event Selection . . . . . . . . . . . . . . . 102

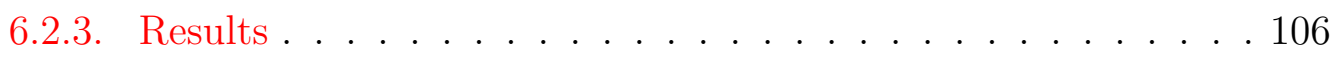

6.3. Conclusion . . . . . . . . . . . . . . . . . . . . 108

7. Data Analysis: Dark Photon Search 111

7.1. Introduction . . . . . . . . . . . . . . . . . . . 111

7.2. Dark photon production . . . . . . . . . . . . . 111

7.2.1. Event Selection . . . . . . . . . . . . . . . . 112

7.2.2. Wide Angle Bremsstrahlung . . . . . . . . . . . . . . . . . . 114

7.2.3. Summary of Event Selection . . . . . . . . . . . . . . 116

7.3. Statistical Analysis for Resonance Search . . . . . . . . . . . . . . . 124

7.3.1. The Model . . . . . . . . . . . . . . . . . . . . 124

7.3.2. Hypothesis Test . . . . . . . . . . . . . . . . . 125

7.3.3. p-value . . . . . . . . . . . . . . . . . . . . . . . . . . . . . . . . . . .

7.3.4. Profile Likelihood Ratio . . . . . . . . . . . . . . . . . 128

7.3.5. Test Statistics . . . . . . . . . . . . . . . . . . . 128

7.3.6. The Discovery Test Results . . . . . . . . . . . . . . . . . . 131

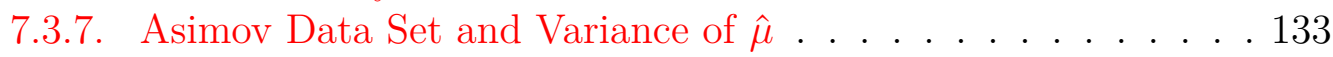

7.3.8. Exclusion Limits . . . . . . . . . . . . . . . . . . . 134

7.4. Conclusion . . . . . . . . . . . . . . . . . . 135

$\begin{array}{ll}\text { Summary } & 139\end{array}$

$\begin{array}{lc}\text { Résumé en Français } & 143\end{array}$

A. Acceptance Parametrization of the ECal $\mathbf{1 4 9}$

A.1. Coordinate Systems . . . . . . . . . . . . . . . . . . . . . . 149

A.2. Parametrization of The ECal Acceptance . . . . . . . . . . . 150

A.2.1. Results . . . . . . . . . . . . . . . . . 153

$\begin{array}{ll}\text { Bibliography } & 155\end{array}$

$\begin{array}{ll}\text { Acknowledgments } & 165\end{array}$

$\begin{array}{ll}\text { Abstract } & 169\end{array}$

$\begin{array}{ll}\text { Résumé } & 169\end{array}$ 



\section{Introduction}

"Everyone is a moon, and has a dark side which he never shows to anybody."

Mark Twain

Our understanding of fundamental interactions and of the evolution of the universe can only describe a fraction of the content of the universe. Dark matter and dark energy were introduced to cover the gaps but remain elusive to our experimental searches. The dark photon idea has independent motivations in theoretical particle physics and cosmology. New symmetries were introduced to solve open questions in the Standard Model, which often involve a hidden sector with its hidden matter states and interactions. The dark photon is one of the possible bridges to link the matter in the visible world to the matter in the hidden sector. Many dark matter candidates inhabit in such hidden sector.

The work presented in this thesis is devoted to the search for a dark photon in the Heavy Photon Search (HPS) experiment at JLab, Hall B. JLab is an accelerator facility with a continuous electron beam of energy from 1 to $12 \mathrm{GeV}$. Dark photons are expected to be produced through a Bremsstrahlung-like process, with the highintensity electron beam of JLab impinging on a tungsten target.

The dark photon production, followed by its dielectron decay, is a rare event amongst a copious amount of QED background. The HPS detector is equipped with an electromagnetic calorimeter and a silicon vertex tracker to maximize the dark photon search efficiency. HPS had a successful test run in 2012 and took data in engineering runs in 2014-2016. The experiment is searching for a dark photon via a resonance search in a large interval of masses and couplings. The work presented in this thesis is focused on the blind analysis of a fraction of the 2015 data, where the silicon vertex tracker was placed at $1.5 \mathrm{~mm}$ from the beam line.

The first chapter is discussing the theoretical background and the motivation for a dark photon detection. The worldwide experimental search for dark photons are reviewed in the second chapter. The third chapter is devoted to a presentation of the HPS detector and the fourth chapter to the detector performances, in particular, for the electromagnetic calorimeter, which was one of the focuses of my work. I study the QED background with Monte Carlo simulations in the fifth chapter. The Moller scattering data are analyzed to understand the detector mass resolution in chapter 6 . The seventh chapter describes the dark photon data analysis, where the $e^{+} e^{-}$pair selection and the statistical analysis of the invariant mass spectrum are presented. 



\section{Physics Motivation}

\subsection{Introduction}

The origin of the universe holds the keys to many problems of modern physics. In the frame of our current understanding of nature, when we trace the evolution of the universe, we observe macroscopic structures emerging from microscopic scale physics. Thus a fundamental study of matter best reveals the core connection of the smallest and the largest in nature.

The concept of matter as a basis of the observable world, and our interaction with it, was introduced by philosophers as early as the 7th or 6th century BC. In Ancient Greece, the founders of materialism, the thinkers of the Milesian school, created an essentially new abstraction about the nature of matter, putting forward universal principles (instead of mythological figures as before) as the underlying cause of its creation and destruction.

Since then, the notion of matter and the understanding of the structure of the universe has evolved significantly thanks to more complex and more developed mathematical abstractions, which allow one to obtain more profound insights to address the same essential questions. The successes of the standard theories of particle physics and cosmology, in parallel with the astronomical observations of the last few decades, brought out challenging problems for these fields. In particular, both in particle physics and in cosmology, experimental observations and theoretical considerations strongly hint at the presence of additional matter states. Detected only through its gravitational effect, this undiscovered matter domain is referred as dark matter (DM).

Attempts are made in both fields to explain the dark matter phenomena, either within its gravitational abstraction or within particle physics. Fig. 1.1.1 shows the landscape of models hypothesized as a solution to the DM problem. The number and complexity of the proposed theories makes it difficult to offer a full overview, so only the models most relevant to this thesis will be presented in this chapter.

The theoretical motivation for a dark photon search has two origins, one coming from cosmology and the other one from particle physics. The following sections are devoted to a brief presentation of the challenges modern theoretical physics is facing in order to build a comprehensive theory for DM observations in cosmology and addressing the problems of the Standard Model (SM) in particle physics. 


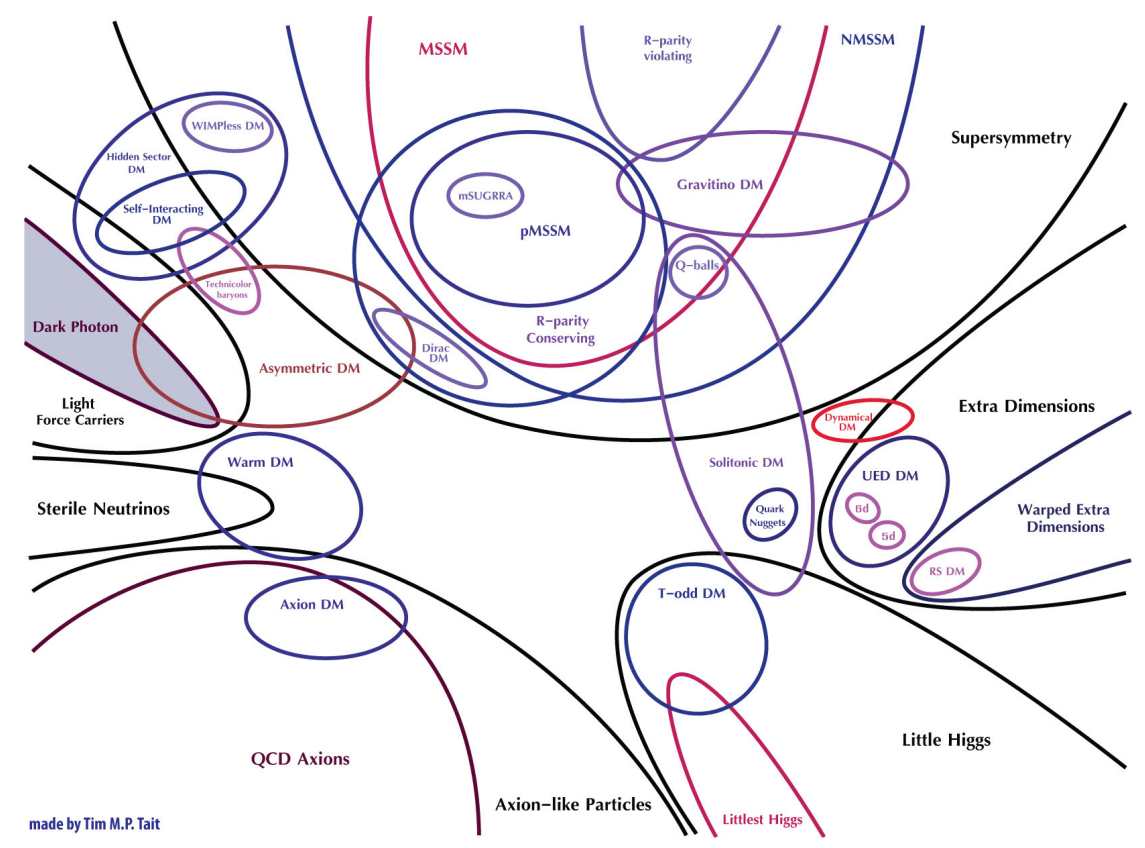

Figure 1.1.1.: The landscape of particle DM candidates [1]. The regions within lines correspond to the suggested theories and the overlapping parts symbolize their cross-relations.

Experiments world-wide are searching for proposed DM candidates, see Fig. 1.1.2. Different experiments employ different detection methods approaching the issue from multiple angles. Indirect searches, using for instance gamma-ray and neutrino telescopes, look for resonances in cosmic ray energy spectra. Direct searches include collider experiments and experiments aimed at detecting galactic dark matter recoiling off nuclei as it passes through the Earth. In this chapter, I will focus on the theoretical basis of the dark photon and present the experimental considerations for dark photon searches in fixed target experiments, in particular for the HPS experiment.

\subsection{Matter in Cosmology}

Until the last few decades, the observations in cosmology were explained by visible matter. It was in the late 19th century and in the beginning of the 20th century, that the first significant deviations from the expected behavior of the stars were detected. The studies of the rotational speed of the stars in the galaxies revealed that the stars are moving too fast, considering the observable luminous matter. The first written remark about this is by the French mathematician and physicist Jules Henri Poincaré in his book "The Milky Way and Theory of Gases" referring to Lord Kelvin's lecture in Franklin university, where from studying the velocity of the stars in the galaxies, he concluded "many of our stars, perhaps a great majority of them, may be dark 


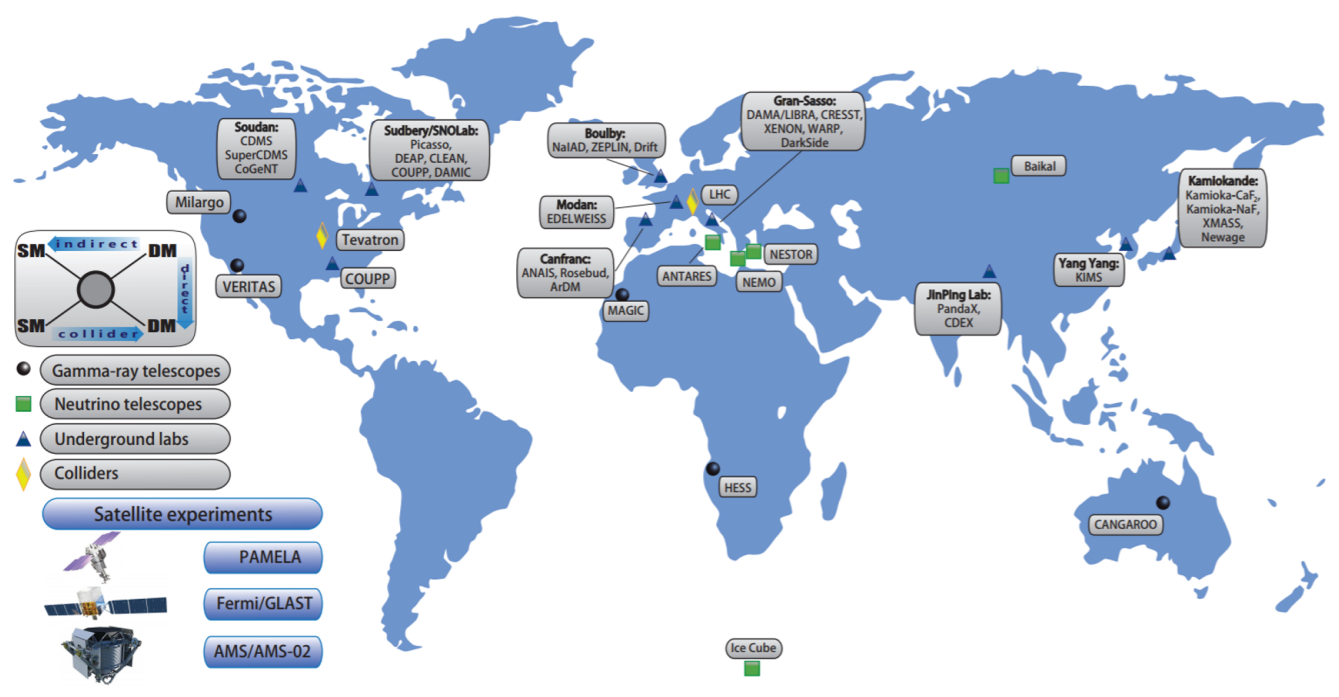

Figure 1.1.2.: A geographical overview of the physics experiments engaged in the search for dark matter [2].

bodies". Poincaré actually calls them in the book "matière obscure" [3]. After that, more and more similar observations were reported. In 1932, another observation of this discrepancy was reported by J. H. Oort. He measured the radial velocity of stars in our galaxy and found that they are moving too fast, but he thought it could be explained by dim stars or by more mass around the center of the Milky Way, hidden from direct observations by bright luminous stars [4]. The term dark matter was introduced by the Swiss astronomer F. Zwicky. A year after J. H. Oort, he published an article about his observations of the Coma cluster mass based on the amount of light radiated. He also calculated the velocities of galaxies and found the same large discrepancy with respect to theoretical calculations. About two orders of magnitude more mass was needed to account for such velocities and Zwicky concluded that there must be additional invisible matter - dark matter [5]. However, these early observations did not get much attention in the scientific community, because either low precision detectors were making the results erroneous and untrustworthy or simply because there was not enough mathematical power to address these issues. Between the 1930s and the 1970s similar measurements were reported and several types of observations also confirmed in parallel the lack of observable matter that should be present given the observed gravitational effects. Thus, by the end of 20th century, it was clear that the physics of the visible part of the Universe was not enough to explain all the observations. The three main evidences are the abnormal rotational speed of stars and galaxies, the excessive gravitational lensing maps and the Bullet Cluster observation. 


\subsubsection{Rotational Curves of Galaxies}

In 1970, V. Rubin and W. Kent Ford Jr. published an article titled "Rotation of the Andromeda Nebula from a Spectroscopic Survey of Emission Regions" [6], hinting to an excess of gravity in the outskirts of the Andromeda galaxy, where the rotational curves $^{1}$ of the Andromeda Nebula were studied. It was found that the stars and gas in the spirals are moving faster than the calculations accounting for the visible mass in the galaxy showed. This was the first robust observation of the effect, confirmed later for many other spiral and elliptic galaxies, see Fig. 1.2.1. The hypotheses to describe the observation are based on the presence of invisible matter, creating excessive gravitational effect, or the modification of the theoretical framework of the gravitational field. Soon after V. Rubin published her first results, various theories were proposed. One of the most successful among these is the Modified Newtonian Dynamics (MOND), attempting to modify the known Newtonian laws to explain the unorthodox rotational curves of the galaxies. Since then, this theory has been constantly updated to fit new data from not only spiral but also elliptical galaxies.

A more elaborate approach is the theory of Emergent Gravity (EG). In his recent efforts, E. Verlinde describes the rotational curves of galaxies and some other astrophysical observations, where gravity is an emergent phenomenon. In the theory of general relativity, gravity is essentially a property of the geometry of the spacetime continuum of our Universe. Verlinde, however, suggests a different approach. Drawing upon the key insights gained from work in black hole physics, information theory, and string theory, Verlinde gives an estimate of "dark gravity" [7]. Emergent Gravity can be considered as a base for the modification of Newtonian dynamics in MOND, as the Tully-Fissher relation used in MOND, can be derived in the framework of emergent gravity (see section 4.4 in [7]). It is worth noting that, while both theories fit the data with the same formula of gravitational force versus distance, EG fit to the rotational curves has no free parameter. Lately, weak lensing (see next section for description of lensing) was successfully tested for this theory [8]. However, further development is necessary to explain other observations in cosmology including cosmic background radiation and inflation.

\subsubsection{Gravitational Lensing and Bullet Cluster}

In his 1937 article, Zwicky [10] suggested several ways to measure the mass of a cosmological object, amongst which he, for the first time, proposed to use gravitational lensing. Light bending was predicted even in Newtonian dynamics. Isaac Newton wrote about "Bodies act upon Light at a distance, and by their action bend its Rays" in the 1st Query of his book "Opticks" first published in 1704 [11]. However, the first accurate calculation was given by Einstein in the framework of his General

\footnotetext{
${ }^{1}$ The speed with which stars and gas in a galaxy revolve around the center of the galaxy as a function of their distance to the center.
} 

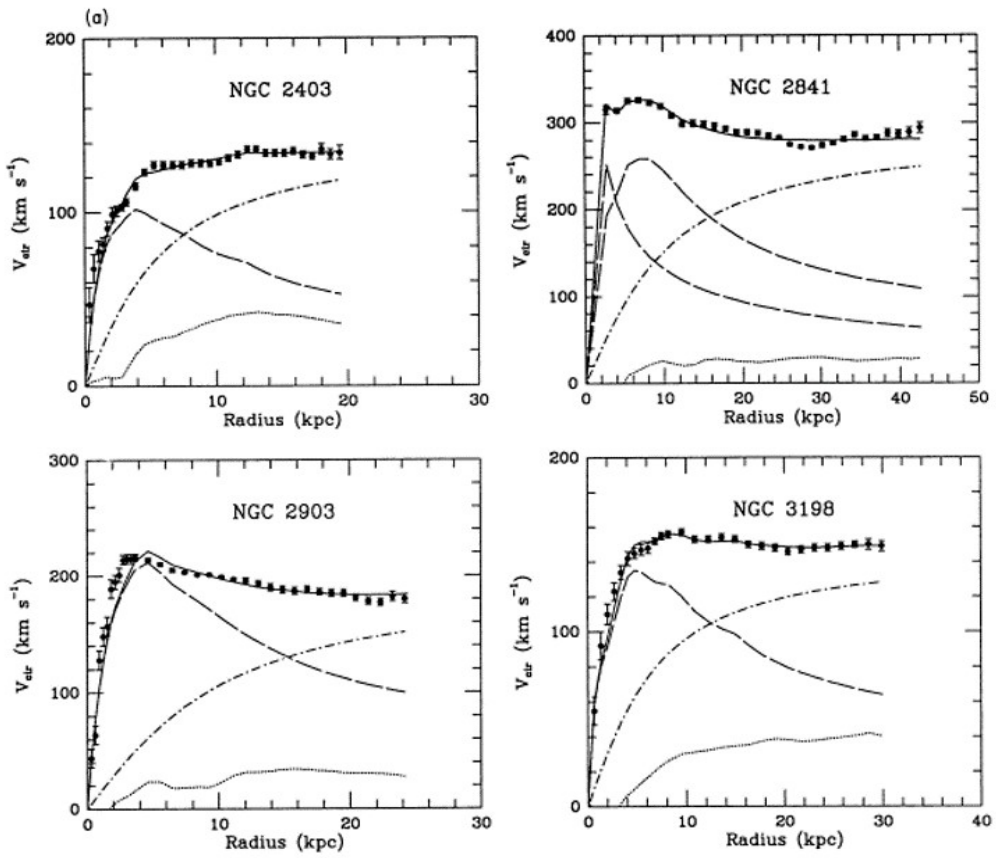

Figure 1.2.1.: The orbital speeds of visible stars or gas in different galaxies as a function of their radial distance from the galaxy center. The data is fitted by a three-parameter model (solid curve) with dark matter (dash-dotted), gas (dotted) and luminous matter (dashed) contributions [9].

Relativity theory. This was experimentally confirmed by Eddington in 1919 [12], while observing the distortion of the light of stars passing close to the Sun. The light from a distant source passing at a distance $r$ of a gravitational source of mass $M$ is being deflected by an angle $\theta$ :

$$
\theta=\frac{4 \mathrm{GM}}{\mathrm{r} c^{2}}
$$

where $G$ is the universal constant of gravitation and $c$ the speed of light. The first such measurement was done in 1979 by Dennis Walsh, Bob Carswell and Ray Weymann [13] discovering the gravitational lensing phenomenon. Gravitational lensing allows for the discovery of mass concentrations that are entirely dark otherwise, making gravitational lensing mapping a unique tool in the search for dark matter. The first application using this method to estimate the absolute mass of a galaxy cluster [14] showed results consistent with the assumptions of dark matter accumulated within galactic clusters along with ordinary matter .

\subsubsection{Bullet Cluster Observation}

Bullet Cluster is the "nickname" given to the system 1E 0657-56 consisting of two colliding galaxy clusters located 3.7 million light years away. Its name is coming 
from the shock-wave $\mathrm{X}$ ray image resembling a bullet, as can be seen in Fig. 1.2.2. This representation consists of three images. The X-ray image in pink shows the distribution of two hot clouds of gas of the clusters after the collision. In blue is the distribution of the mass in the clusters mapped with gravitational lensing of background galaxies. Finally, the white or yellow colors show the luminous matter.

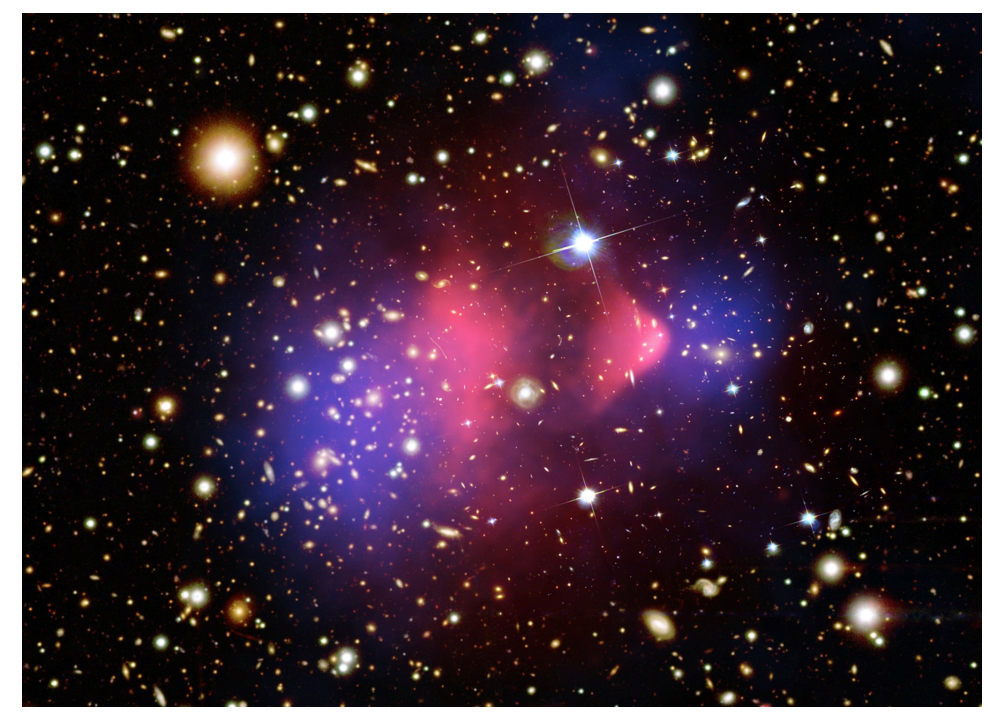

Figure 1.2.2.: The galaxy cluster 1E 0657-56 or "bullet cluster" [15] formed after the collision of two large clusters of galaxies. Pink: $\mathrm{X}$ ray image showing the distribution of gas in the clusters [16]. White or yellow: the optical image of stars and galaxies. Blue: gravitational lensing map, where most of the matter of the system is concentrated [17].

Not only the mass from the stars and the gas clouds represents only a fraction of the total matter observed with lensing, but the clear distinction of the "pink" gas clouds from the blue blobs is uncanny. It shows that this dark non-illuminant substance did not interact with the gas cloud nor with the blue cloud of the other cluster. It passed through unaffected whilst the pink clouds of hot gas were distorted in the collision. This observation is considered the most direct observation of dark matter, so far favoring a particle-based dark matter.

MOND, alternatively, claims to partly account for the dynamics in the clusters, leaving only a factor 2 of mass unexplained [18, 19], which could be justified by ordinary matter in form of black holes or some other non-luminous form. There are many theories explaining one of these phenomena, but often missing out on another. The most accepted model today to explain all these features of the Universe is the Lambda Cold Dark Matter $(\Lambda \mathrm{CDM})$ theory, that I will describe in the next section. 


\subsubsection{Evolving Universe in the Big Bang Model}

In the 1940s, George Gamow et al. introduced their ideas about the cosmological origin of the abundance of the elements in the Universe. According to the Big Bang model, the universe was "born" about 13 billion years ago. It started expanding from an incredibly hot, dense state (after inflation) [20]. Passing through different phases of cooling, it continues to expand to this day. This evolution has been imprinted in a relic background radiation predicted in 1948 by Alpher and Herman [21] and discovered in 1964 as the microwave background radiation [22]. The detection of the cosmic microwave background (CMB) made the Big Bang model a favorite amongst the other models. This discovery boosted further developments of the model revealing the homogenous, flat and isotropic nature of the CMB that conflicted with the understanding of the model at that stage. This was solved within the Inflationary Universe model which essentially describes this incredibly hot and dense state, explaining the first moments of time. Nowadays the Big Bang refers to the initial singularity that triggered the inflation without addressing its origin, be it the default state of an outer universe or some random fluctuation. Fig. 1.2.3 illustrates the history of the Universe based on the Big Bang model and its different evolution stages that are described below.

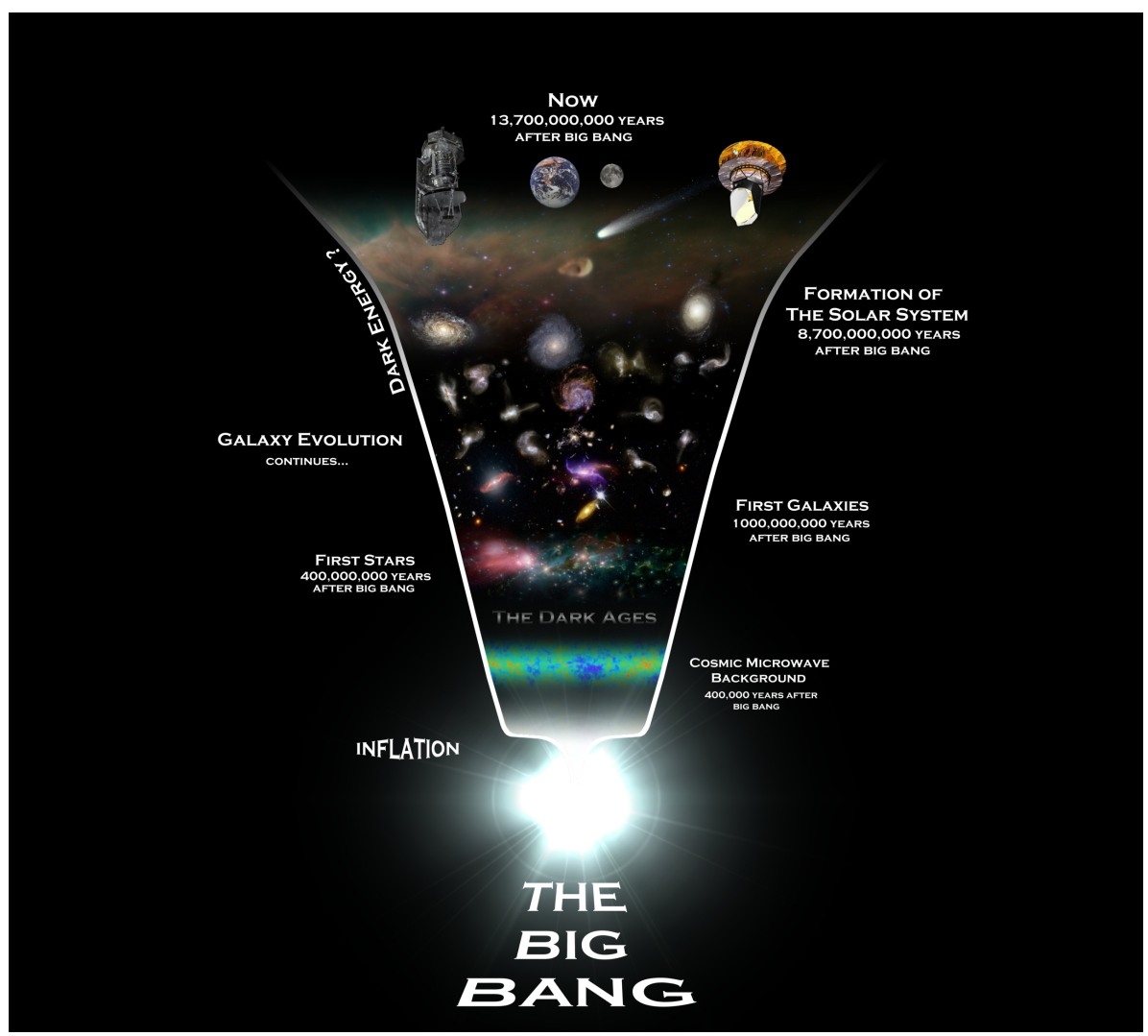

Figure 1.2.3.: The timeline of the evolution of the Universe. 
During the first $\sim 10^{-36}$ to $10^{-33,32}$ seconds, the universe went through an extremely fast expansion from subatomic to macroscopic size. This period is called inflation. As a result, it flattened the original inhomogeneities and made the universe the homogenous, flat and isotropic place which we observe today [23]. Quantum fluctuations caused density fluctuations, which later played the role of cradles or seeds for structure formation. This period of the evolution of the universe is described by the Inflation Theory.

The inflationary period ended leaving the primordial universe bubble, blown up $10^{26}$ times of its original size, to cool down with an accelerated expansion, but much slower than during the inflation. The early universe at this stage was very hot and dense, best described as a plasma, where particle-antiparticle pairs were being created and annihilated. Then the universe continued to expand and cool down, such that the pair formation of some particles became more and more unlikely. At temperatures around $\sim 10^{12} \mathrm{~K}$ there was no more proton-antiproton formation, and at $\sim 10^{9} \mathrm{~K}$ electron-positron pair formation stops. These temperatures are known as the freeze-out temperatures for given particle species. The creation of the regular matter in the universe is explained by this process of Baryogenesis that happened at a scale of one second after the Big Bang, generating matter-antimatter initial asymmetry, in a way that is not yet understood.

Between a 100 to a 1000 seconds after the singularity, the universe cooled down enough to allow for the protons and neutrons to stick together and form stable nuclei. Since free neutrons are not stable, the number of created nuclei was limited by the neutron lifetime which is approximately 15 minutes. At the same time, the space between particles was expanding, putting another restriction on the number of interactions between protons and neutrons. In the standard cosmological model, this period is described by the Big Bang Nucleosynthesis (BBN). This theory predicts the abundance of light elements in the universe. Heavier elements are formed in the core of stars through nuclear fusion reactions, but in contrast to stellar nucleosynthesis, primordial nucleosynthesis is driven by non-equilibrium processes. The lighter elements like deuterium, helium isotopes ${ }^{3} \mathrm{He},{ }^{4} \mathrm{He}$, lithium isotope ${ }^{7} \mathrm{Li}$ and beryllium ${ }^{7} \mathrm{Be}$ were created in a radiation-dominated environment with high entropy $\left(10^{9}\right.$ photons per baryon and many neutrons) within the first few minutes of time. The amounts of each of these nuclei in the cosmos depend crucially on the conditions in the early universe, in particular on the balance between baryonic matter (protons and neutrons) and non-baryonic matter (neutrinos and exotic particles, including dark-matter particles). Therefore, by measuring the abundance of nuclei of these elements today, we obtain constraints about the dark-matter particle properties and their abundance during the nucleosynthesis age.

\subsubsection{WIMP miracle}

The properties of the particles composing dark matter can be inferred based on simulations from the structure formation in the early universe to the star formation. 
Modern observations of the universe constrain these simulations based on ordinary matter interactions and hypothesized dark-matter particles with specific properties. These calculations, using the matter density fluctuations from the CMB, show that without introducing non-baryonic matter, the initial fluctuations do not have enough power to grow into galaxies and the clusters of galaxies we observe today. Moreover,
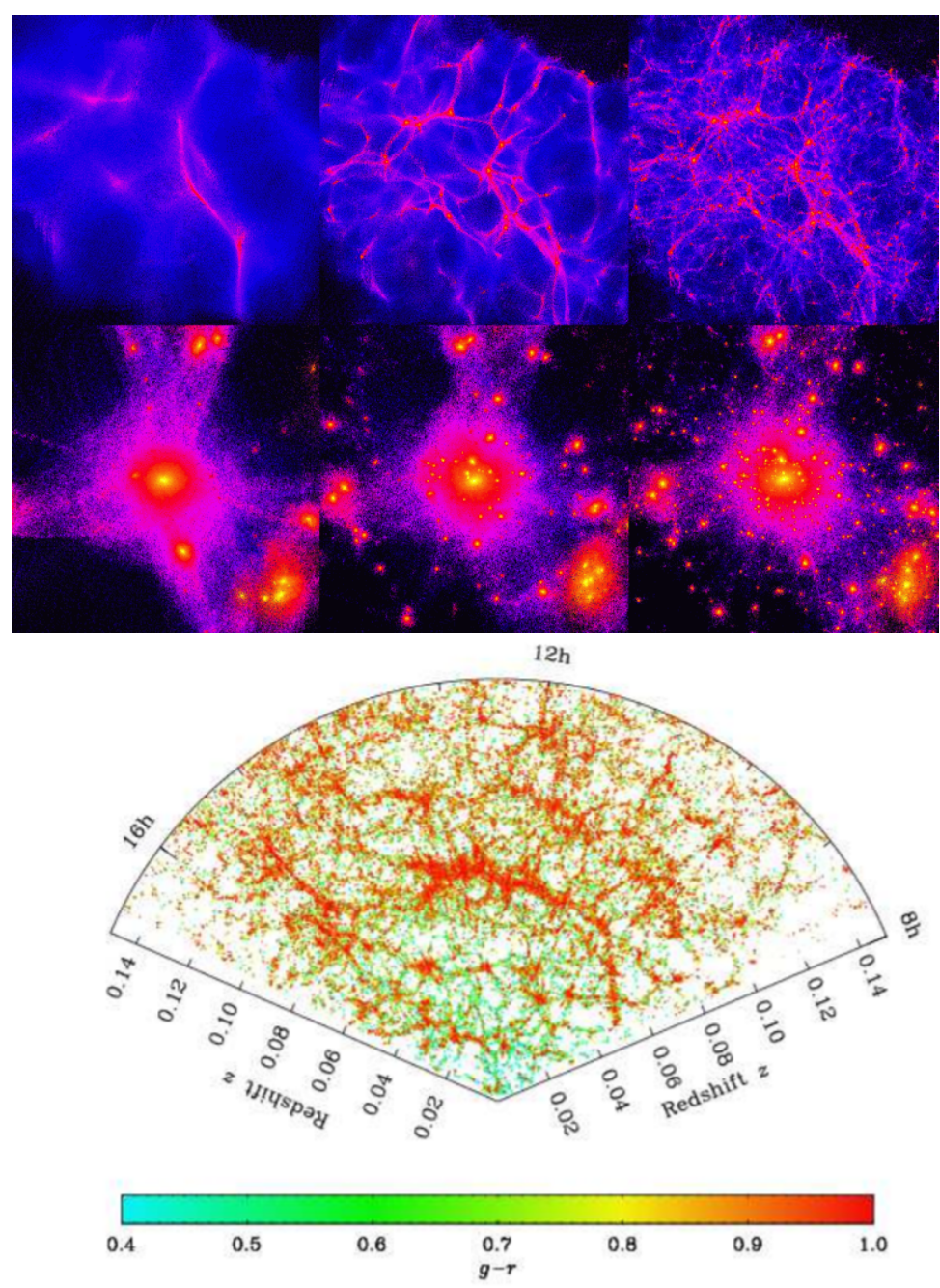

Figure 1.2.4.: Top: from left to right: hot, warm and cold dark matter simulations (credit ITP, University of Zurich). Bottom: Large scale spatial distribution of the galaxies in the SDSS sky survey as a function of redshift, from experimental observations [24].

these simulations offer constraints on the thermodynamic properties and the free distance over which dark matter can travel. As shown in Fig. 1.2.4, different scenarii have been identified for the properties of dark matter:

- Hot dark-matter scenario: Dark-matter particles have light masses and relativistic velocities (like neutrinos). Due to their speed, the small density 
fluctuations are smoothed out and baryons are being congregated around bigger fluctuations. The structure formation is evolving in a "top-down" way, meaning it is slow and results into late galaxy formation and small structure extinction.

- Cold dark-matter scenario: Non-baryonic DM particles are moving slowly due to their large masses $(\sim 100 \mathrm{GeV})$, thus the small fluctuations are incorporated into structure formation, encompassing the ordinary matter after decoupling (baryons and photons decouple). This allows for early structure formation and hierarchical "bottom-up" evolution, where big structures such as galaxies and clusters are formed from stars and galaxies, with small systems forming from large scale systems fragmentation.

- Warm dark-matter scenario: In this case the particles of dark matter have intermediate velocities with masses at the $\mathrm{keV}$ or $\mathrm{GeV}$ scale. At temperatures below the electroweak scale, they interact only weakly with SM particles by natural mixing with neutrinos. However, the mixing probabilities are small, meaning they are not produced in very large quantities due to the freezeout halting production before thermal equilibrium is reached. In this case, the structure formation is in a "bottom-up" way, and the resulting structure formation pattern is similar to our observations.

In $\Lambda$ CDM, the correct abundance of dark-matter is obtained from the cold dark matter model with a self-annihilation cross section of $\sim 3 \times 10^{-26} \mathrm{~cm}^{3} \mathrm{~s}^{-1}$ and mass $\sim 100 \mathrm{GeV}$ for dark matter particles. Particles with similar properties are predicted by simple super-symmetric models. This coincidence was called the WIMP (weakly interacting massive particle) miracle.

\subsubsection{The Quantity of Matter in the Universe}

After the nucleosynthesis epoch, in the matter-dominated universe, ordinary matter consists of nuclei, photons and electrons. Around 380000 years after the Big Bang, the first neutral atoms are formed and the photons are no longer in thermal equilibrium with other charged particles. The universe becomes transparent to light, producing the earliest radiation that we can observe. Because space continued to expand, the wavelength of this light became stretched and is now observed as the CMB present in every direction in space.

The oscillation in the power spectrum of the CMB, illustrated in Fig. 1.2.5, probes different epochs of the observable universe's evolution. The contributions of each component of the universe are sensitive to the microwave radiation at various stages of its evolution and their imprint is preserved in the wiggles of the power spectrum as a unique fingerprint.

Another way of estimating the density of matter and energy in space is coming from Type Ia supernovae studies. In combination with the previously discussed 


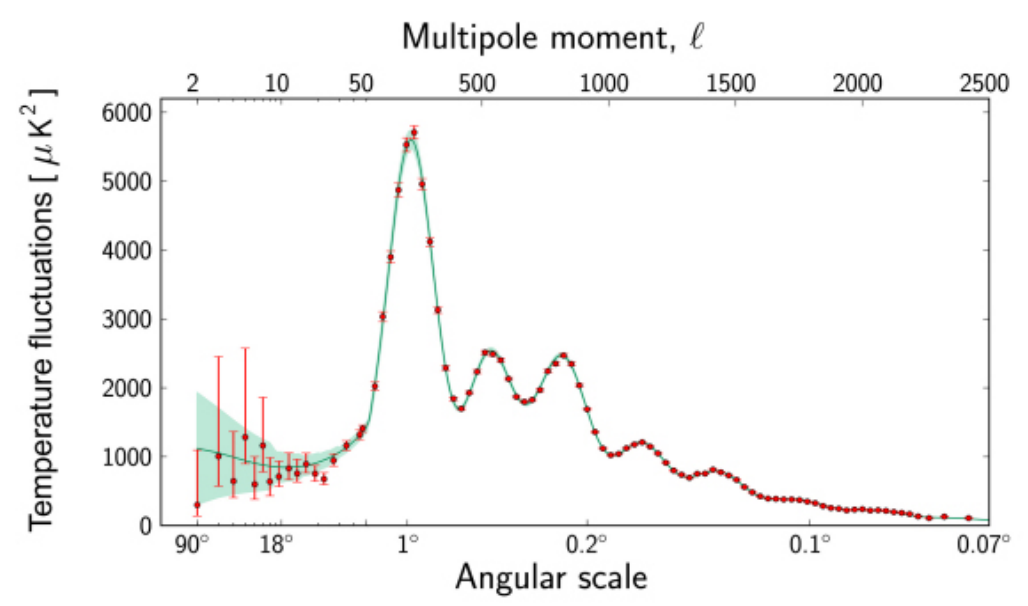

Figure 1.2.5.: Power spectrum of the CMB (Planck data) [25].

measurements, it constrains both $\Omega_{\Lambda}$, the density of the energy in the universe, and $\Omega_{m}$, the density of matter (both light and dark), shown in Fig. 1.2.6.

Taking into account the measurements obtained from the Big Bang nucleosynthesis and the CMB studies, the content of the universe consisting of hypothesized cold dark matter, luminescent matter and dark energy can be estimated. As illustrated in Fig. 1.2.7, we are living in an expansion-dominated (dark energy $\sim 68 \%$ ) universe, where most of the matter is hidden in the form of dark matter $(\sim 27 \%)$ and only $\sim 5 \%$ is ordinary matter.

\subsection{Matter in Particle Physics}

Coupled with special relativity, quantum field theory allowed to develop powerful theoretical tools to study particles with many experimental successes. Based on various models and shaped by the experimental observations, the Standard Model (SM) of particle physics is the theory of visible particle matter. Starting from around the 1950s, it evolved into a model categorizing the discovered particles based on underlying symmetries, using group theory. This allowed for a deeper comprehension of the nature of the matter, categorizing known particles in hierarchical groups and predicting yet unknown states. By the end of the 20th century, almost all of these predictions were confirmed, in particular with the Higgs particle detected at LHC in 2012 [29, 30]. Fig. 1.3.1 illustrates the classification of the known elementary particles, based on their properties. Despite the success, some questions remain unanswered within the SM framework. Some of them, most relevant to this work, are mentioned in this section. 


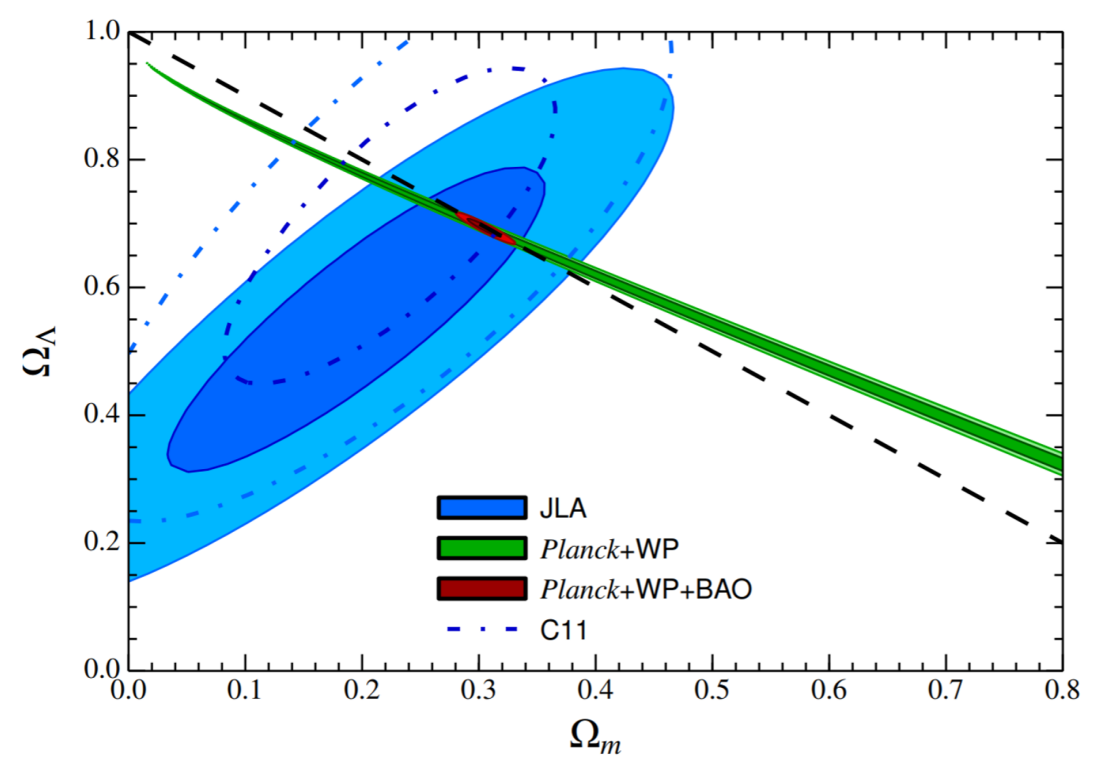

Figure 1.2.6.: The $68 \%$ and $95 \%$ confidence contours (including systematic uncertainty) for the energy $\Omega_{\Lambda}$ and matter $\Omega_{m}$ density in the Universe from three independent measurements [26]. Shown are constraints from Type Ia supernovae (JLA, C11), and WMAP and Planck CMB measurements (excluding/including baryon acoustic oscillations (BAO)). Also shown is a black dashed line which corresponds to the expected values for a flat universe.

\subsubsection{Problems of the Standard Model and Proposed Theoretical Solutions}

- Gravitation - The standard model does not include general relativity within the framework of quantum field theory. The existing attempts to integrate the gravitational force into the SM run into problems at the Planck length where quantum corrections of the gravitational field become important.

- Hierarchy problem - In the SM, elementary particles gain a mass through the Higgs mechanism which operates at the electroweak scale - about a few hundred $\mathrm{GeV}$. Thus, the resulting particle masses do not extend beyond this scale. Quantum corrections to the effective value of the electroweak coupling from gravity are, however, of the order of $\sqrt{\frac{\hbar c}{8 \pi G_{\text {Newton }}}} \sim 10^{18} \mathrm{GeV} / \mathrm{c}$. In more general terms, for an observable $A$ presented as a sum of $N$ independent perturbative contributions $a_{i}$,

$$
A=a_{1}+a_{2}+\ldots+a_{N}
$$

all $a_{i}$ contributions are expected to be smaller or comparable to $A$. This intuitive expectation can be violated in quantum field theory, when the measured effective value of a parameter is vastly different from the value in the 


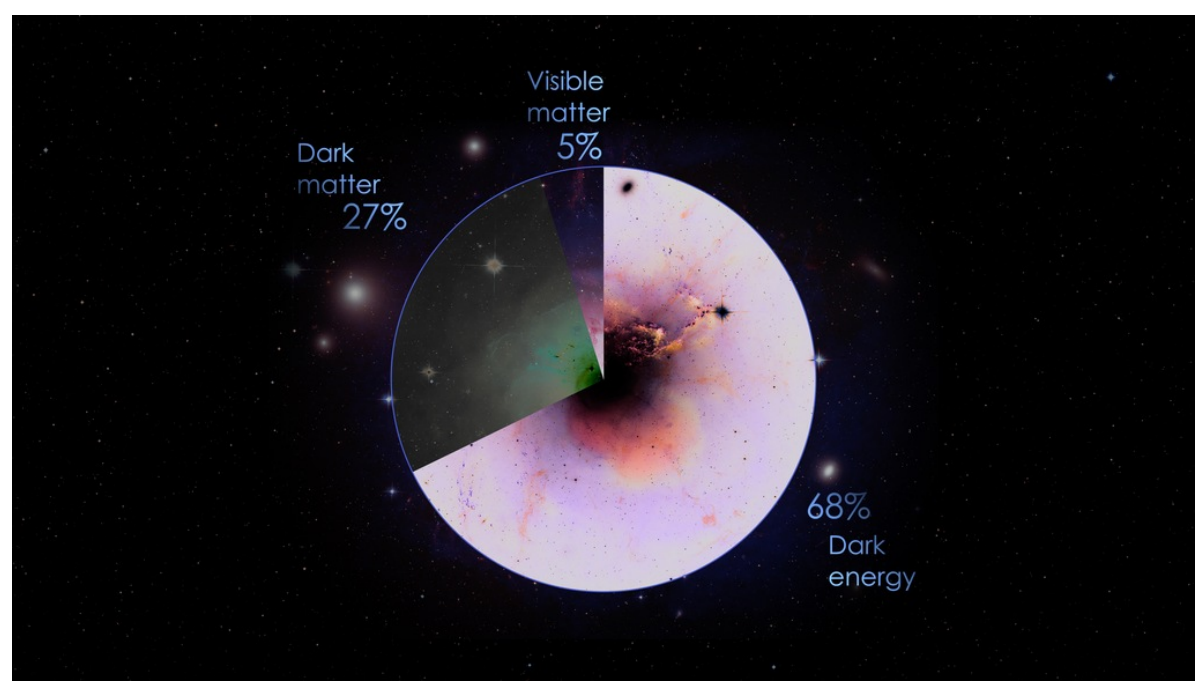

Figure 1.2.7.: The content of the universe [27].

Lagrangian. The disproportional discrepancy between the scales of the perturbatively corrected and bare value of the parameter in the SM is known as the gauge or hierarchy problem. It brings up a number of fine-tuning and naturalness issues in the SM as well. To get meaningful physical results, renormalization techniques are used, but these do not arise naturally and can be considered unphysical. While renormalizable field theories can be accepted, the origin of the scales remain largely unexplained within the SM.

Assuming a fermionic/bosonic symmetric partner to every SM particle, supersymmetric (SUSY) models can solve the hierarchy problem depending on the scale of the symmetry breaking. Experiments at CERN failed to find the lightest SUSY partners thus pushing the mass limit of the lightest SUSY particle to the $\mathrm{TeV}$ scale. While solving the global scale discrepancies, the high scale SUSY creates so-called little hierarchy problem. Some questions of the hierarchy problems within SUSY framework are discussed in [31].

- Strong CP problem - The lack of CP violation in QCD is a mystery, as there is no reason for this symmetry to be conserved. Yet, no violation has been detected in experiments with current experimental bounds on the $\bar{\theta} \mathrm{CP}$ violation parameter of the order of $\mathrm{O}\left(10^{-10}\right)$.

One of the solutions to this problem is to add an additional global chiral PecceiQuinn symmetry $\mathrm{U}(1)_{P Q}$ that is spontaneously broken [32] at some scale $f_{a}$. Instead of a static $\bar{\theta}$ parameter, we have a dynamical scalar field ensuring $\mathrm{CP}$ conservation by introducing in the theory an axion. The axion is a light, very weakly coupled, long-lived pseudo-Nambu-Goldstone boson, which is a very attractive DM candidates [32, 33]. Such fields and particles are also natural in Grand Unified Theory (GUT) models such as string theory.

- Matter/antimatter asymmetry - The Standard Model does not accom- 


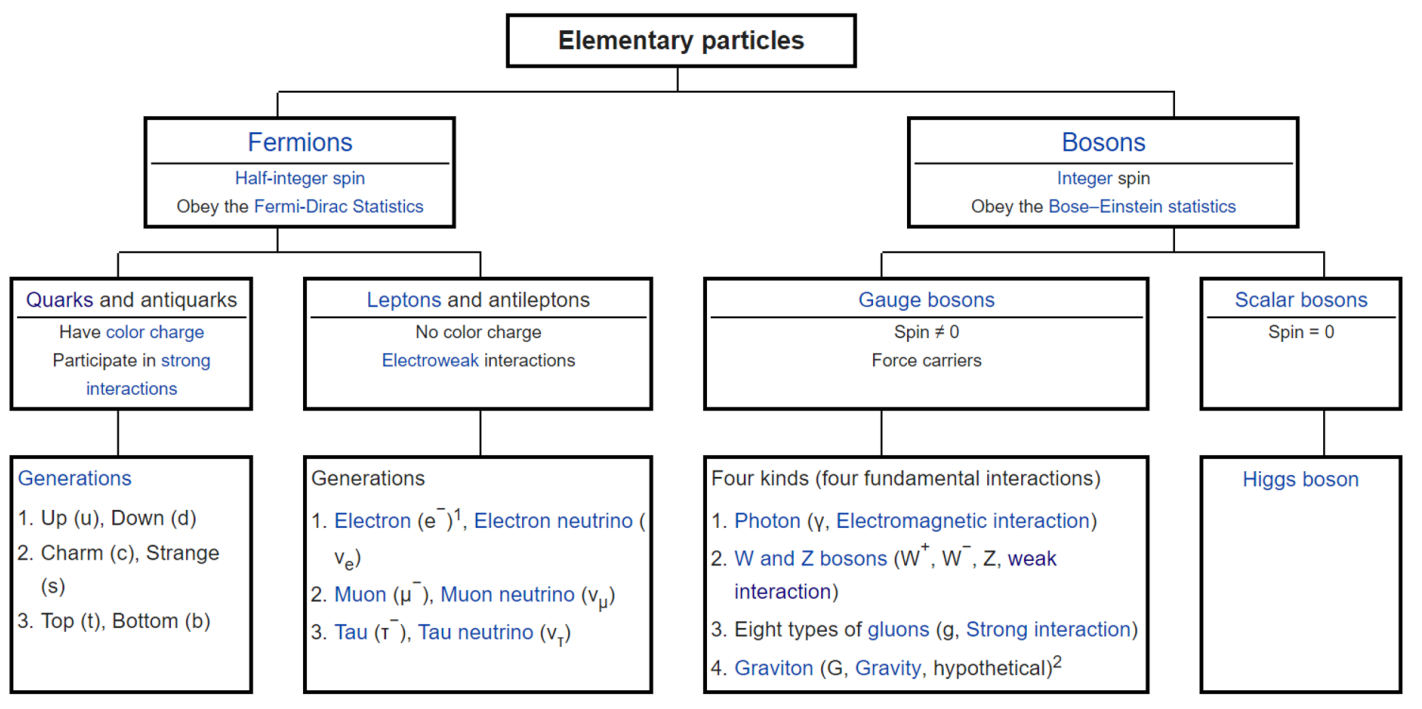

Figure 1.3.1.: Elementary particles in SM [28].

modate the dominance of matter over antimatter in the observable Universe. Three Sakharov conditions are necessary to resolve this issue: baryon number violation, $\mathrm{C}$ and $\mathrm{CP}$ symmetry violation, and out-of-equilibrium interactions [34].

The discovery of the Higgs boson allowed to study the mechanism of the initial asymmetry generation with "Higgs Relaxation" [35], where Sakharov conditions are generically met via the Higgs field. During inflation, the Higgs field relaxes from a quasistable additional minimum or from randomly distributed vacuum expectation values to its vacuum state after inflation. From the initial baryon - anti baryon asymmetry generated in such a way, the Sakharov principles take over by letting the small amount of baryon matter evolve, creating our observable universe, while the rest of hot baryonic pairs annihilate into photons. It was claimed by Rubakov and Shaposhnikov [36] that the problem of baryonic asymmetry of the universe can not be solved in the framework of the Standard Model [37] .

Alternatively, it was suggested that there are regions of the universe with antimatter separated from our matter-dominant universe. On the boundary with such a neighbor, photon radiation would occur from annihilation processes, but observations so far failed to detect such characteristic radiation space [38].

- Vacuum Stability - The true values of the top-quark mass and Higgs mass within the Standard Model define the shape of the Higgs potential. Current values hint to a possibly metastable vacuum assuming no other physics beyond SM. This suggests minima other than the current $246 \mathrm{GeV}$ vacuum expectation value associated with the Higgs boson, leaving a non-zero probability for our vacuum to tunnel into a more stable state with lower minimum. The most detailed calculations performed after the Higgs discovery concluded that even 
though all the evidences point to a metastable vacuum, the limits on the measured values of the SM parameters are consistent with the stable scenario at least up to the Planck mass scale [39]. More precise measurements of these parameters might shed more light on this issue. Other studies suggest that during the inflationary stage, the "regions" with the Higgs in its true minimum would shrink to a point, since anti-de Sitter (AdS) space contracts. These points would be diluted and thus neutralized by the expansion or be irrelevant to our universe [40].

- Fine tuning problem - This problem reflects the sensitivity of SM to the numerous constants arising in the theory such as the fine structure constant, which is the square of the electron charge expressed in Planck units that defines the scale of the charges of elementary particles, the mass ratios of elementary particles (or Yukawa couplings), the strong force coupling constant, the gravitational coupling constant, the parameters of the CKM (Cabibbo-KobayashiMaskawa) matrix describing quark oscillations, and the PMNS (PontecorvoMaki-Nakagawa-Sakata) matrix describing neutrino mixing. All these parameters of the SM are adjusted accurately, to agree with the measurements. The small variations of these values have a large impact on the outcome of the model, while the tuned values of the parameters are not justified by any known underlying mechanism. This sensitivity of the model to its parameter values is called the fine tuning problem.

- Anomalous magnetic moment - This refers to the deviation of the measured lepton magnetic moment from the predicted theoretical value. This topic is discussed in more details in sec. 1.4.3.

- DM problem - As a theory of matter, the Standard Model harbors an embarrassing problem in that it does not describe $80 \%$ of the matter in the Universe, according to the $\Lambda$ CDM model of cosmology.

Attempts to resolve these issues inspire many theories. Most of them are originating from the efforts to restore the broken symmetries of the SM in a search of higher symmetries controlling the interactions at higher scales, where a naturally unified force guides the physics. String theory is a candidate for such "Theory of Everything", describing nature from subatomic to large scales. Being based on the framework of quantum field theory, it has many of the same flaws, but string phenomenology is widely used in various fields of physics as a basic abstraction for solutions to concrete problems (e.g. black-hole physics, nuclear physics, quantum gravity, condensed matter physics etc). 


\subsection{Hidden Sector}

The most challenging puzzle presented to theoretical particle physics in the $1950 \mathrm{~s}$ was the $\theta-\tau$ problem $^{2}$. It was resolved by the discovery and experimental confirmation of parity violation in weak interactions of $\beta$-decays, and it triggered a series of articles considering the consequences of $\mathrm{P}$ violation in the physical world. In their article discussing the parity violation, Lee and Yang also point out the possibility of hypothetical right-handed partners of protons [41] that would restore the mirror symmetry. This idea was developed further by Okun, Kobzarev, Pomeranchuk conjecturing CPA symmetry, where A stands for Alice ("Alice in the Wonderland"), leading to hypothetical mirror particles with their own hidden mirror sector interactions and with their own shadowy world structure [42]. Furthermore, they later discussed the (im)possibility of gravitational impact on the ordinary world from the hidden one (surprisingly, as noted in [43], the authors had no idea about the publications by Oort and Zwicky hinting on missing matter.) The mirror particle models were evolved in the following years and ended up deviating considerably from the original idea. The kinetic mixing as an interaction portal with ordinary partners of hidden particles was suggested already along with muonic photons [44] by Okun in 1969 and was inspired from baryonic photons [45]. Later paraphotons [46, 47] were discussed.

In the beginning of the 21st century, motivated by the overwhelming evidence for dark matter, the hidden mirror world caught renewed attention from the theoretical physics community. Several aspects of a possible mirror world were studied, particularly in Grand Unification Theories and String Theory models.

It was shown [48] that the mirror reflection of the particle groups would not necessarily evolve identically to the ordinary world. For example, in the early universe, mirror particle plasma could have a different initial temperature (lower), than the plasma of ordinary matter particles. Such a condition can naturally occur from inflation models. An essential part of the mirror world models is that their interaction with ordinary particles is via gravity only, which provides generically a very weak interaction. This brings about the coexistence of two types of plasma out of thermal equilibrium. Although, it is possible that a weak interaction other than gravity (e.g. kinetic mixing with electromagnetic plasma) as pointed out in [49], might better describe the current abundance of Helium and Deuterium in the Big Bang models. Another condition required by [48] is the adiabatic expansion of two types of matter plasma in a way that leads to low entropy production. This requirement is linked to the initial baryon number asymmetry, necessary for the Sakharov conditions. The mirror photon density in [48] is proportional to the temperature ratio of the mirror particle sector and the ordinary particle sector, which makes it very small $\left(T_{\text {mirror }} \ll T_{\text {ordinary }}\right)$. In contrary, the baryon density can be larger than in

\footnotetext{
${ }^{2}$ Two particles with similar masses and lifetimes, but different spin and intrinsic parity were observed in the experiments, puzzling physicists on whether there are two particles, or whether they are observing two different states of the same particle.
} 
the ordinary world, thus accounting for dark matter if the ratio is $\sim 5$. This model shows that the two different sectors of matter in the universe, while originating from a unified symmetry, could evolve independently developing through different symmetries $[50,51,52]$ and forming "separated" matter domains in the universe which have little to do with one another.

Certainly, a hidden sector provides a fruitful environment to solve many issues of modern physics.

\subsubsection{Portals to The Hidden World}

Adding an additional U(1) symmetry to the SM has been speculated in the context of SUSY models, conceptualized within mirror world formulations, and often in GUT string theory constructions. In the most general terms, we can consider a SM gauge group $G_{S M}$ extended by a non-abelian group $G_{D}$ with light particles that are not charged under the $G_{S M}$ group. In this case, the ordinary particles from the SM can interact with "hidden particles" only through loops of heavy particles, contained in $G_{D}$, that carry charges of both $G_{S M}$ and $G_{D}$. If $G_{D}$ has low energy hidden states, they are expected to be neutral under the full SM gauge group, otherwise they would have been detected in various collider experiments already. The possible interactions between $G_{S M}$ and $G_{D}$ can be categorized based on the type of portal required:

- Scalar portal - It is the most general extension of the SM, where an additional scalar field is assumed with its mediator interacting with the SM Higgs boson. Experimental searches of this mediator are done by studying Higgs decays. This portal is also commonly referred to as the Higgs portal.

- Vector portal - In this case the extension of $G_{S M}$ is performed with one or more vector states coupled to an electromagnetic current, which was first suggested by Okun in 1982 [46]. This involves extending the known landscape of interactions with additional gauge symmetries mimicking QED. In analogy to a "seesaw" neutrino mechanism, if the determinant of the mass matrix of these vector states in such models is zero, then the model has one massless vector state, associated with the photon, and several massive states. These massive states are called paraphotons or sterile photons [53] by Okun. Conjectured as a SM photon "reflection" in the hidden sector, the dark photon can interact very weakly with SM particles through mixing with ordinary photons. Such interactions can be allowed by the kinetic mixing mechanism discussed later. Alternatively, direct tree-level couplings can occur for the dark vector portal interacting with the SM, where some combinations of SM fields are charged under the new $\mathrm{U}(1)^{\prime}$, such as the $\mathrm{U}(1)_{B-L}$ gauge group, allowing for a direct tree-level coupling to dark photons with a small coupling constant [53].

- Pseudoscalar portal - Motivated as a solution to the strong CP problem and dark matter, an additional U(1) Peccei-Quinn global symmetry is added 
with a pseudoscalar mediator, the axion [32].

- Neutrino portal - Assuming an additional light mediator in the SM - the sterile neutrino can explain the neutrino mass problem in the SM. This portal is motivated also from the dark matter perspective. The simulations of the $\Lambda \mathrm{CDM}$ model overestimate the number of small scale structures like satellite galaxies with collisionless dark matter. With the sterile neutrino as a mediator for neutrino-DM interactions, and assuming self-interacting dark matter in the early universe, the decoupling is delayed saving the small-scale halos from collapsing. The sterile neutrino in these models is an exclusive bridge for dark matter and SM particle interactions [54].

\subsubsection{Kinetic Mixing For Vector Portal}

A new $U(1)$ gauge group could be responsible for dark charge interactions in the hidden sector by an analog of the hypercharge interactions in the SM. Dark-photon interactions with the ordinary SM photon could then happen through mixing with the Lagrangian:

$$
\mathcal{L}=-\frac{\epsilon}{2} F_{\mu \nu} F^{\prime \mu \nu}
$$

where $F_{\mu \nu}$ is the field strength tensor, related to the photon field $\gamma$ and $F^{\prime \mu \nu}$ is the field strength tensor related to the dark photon field $\gamma^{\prime}$ :

$$
\begin{aligned}
& F_{\mu \nu}=\partial_{\mu} \gamma_{\nu}-\partial_{\nu} \gamma_{\mu}, \\
& F^{\prime \mu \nu}=\partial^{\mu} \gamma^{\prime \nu}-\partial^{\nu} \gamma^{\prime \mu} .
\end{aligned}
$$

In this case, electroweak symmetry breaking gives rise to an effective interaction between dark photons and fermions:

$$
\mathcal{L} \sim \epsilon e \bar{\psi} \gamma^{\mu} \psi \gamma_{\mu}^{\prime}
$$

where $\epsilon^{2}$ is proportional to the ratio of the dark photon field coupling constant $\alpha^{\prime}$ and the electromagnetic constant $\alpha$ :

$$
\epsilon^{2} \sim \alpha^{\prime} / \alpha
$$

This leads to milli-charged states interacting through a vector boson that could be a linear combination of massive and massless states similar to the SM hypercharge [55]. Also, for dark photon masses larger than the lepton masses, $M_{\gamma^{\prime}}>2 m_{\ell}$, with 
allowed quantum numbers, $\gamma \gamma^{\prime}$ mixing can lead to an observable sharp resonance with a width, in lepton pair production and in decays to hadrons (if in addition $\left.M_{\gamma^{\prime}}>2 m_{\text {hadron }}\right)$ given by [56]:

$$
\begin{gathered}
\Gamma_{\gamma^{\prime} \rightarrow \ell^{+} \ell^{-}}=\frac{1}{3} \alpha \epsilon^{2} M_{\gamma^{\prime}} \sqrt{1-\frac{4 m_{\ell}^{2}}{M_{\gamma^{\prime}}^{2}}}\left(1+\frac{2 m_{\ell}^{2}}{M_{\gamma^{\prime}}^{2}}\right), \\
\Gamma_{\gamma^{\prime} \rightarrow \text { hadrons }}=\frac{1}{3} \alpha \epsilon^{2} M_{\gamma^{\prime}} \sqrt{1-\frac{4 m_{\ell}^{2}}{M_{\gamma^{\prime}}^{2}}}\left(1+\frac{2 m_{\ell}^{2}}{M_{\gamma^{\prime}}^{2}}\right) \times \frac{\Gamma\left(e^{+} e^{-} \rightarrow \text { hadrons }\right)}{\Gamma\left(e^{+} e^{-} \rightarrow \mu^{+} \mu^{-}\right)}\left(E=M_{\gamma^{\prime}}\right) .
\end{gathered}
$$

The branching ratios of the dark photon decay into SM particles are shown in the Fig. 1.4.1.

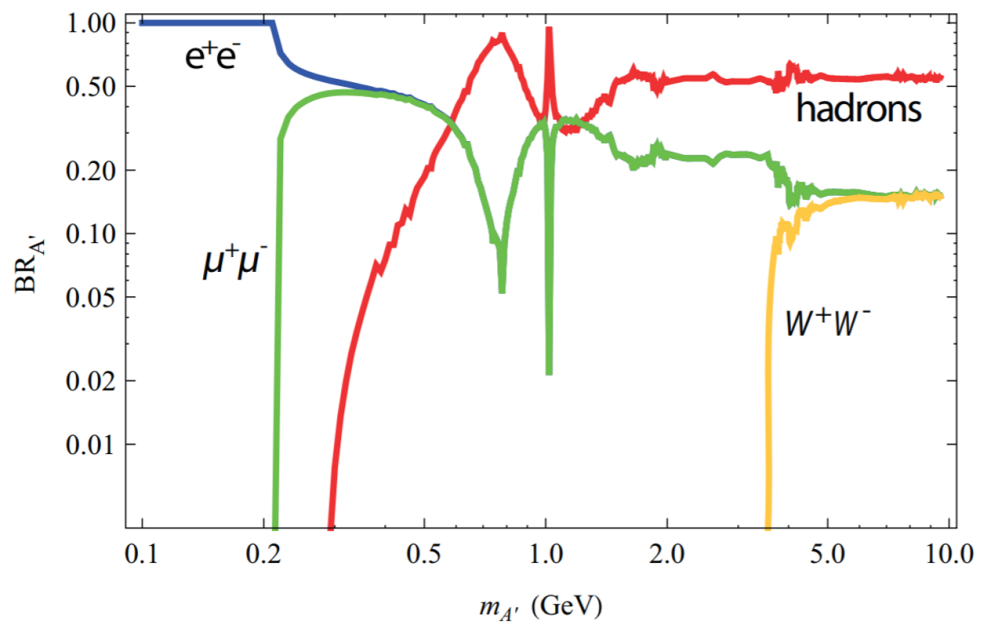

Figure 1.4.1.: The branching ratios of the dark photon decay versus its mass [57].

Continuing the logical extension of the dark sector by analog of the SM's QED, the dark sector can have light states below the dark photon masses. Depending on the scale of its coupling to the dark photon, the visible decays such as to leptons and to hadrons can be suppressed by having the dark photon decay into dark sector particles, $\chi$. For a simple case with additional dark fermions with masses $m_{\chi}$, $m_{\chi}<\frac{1}{2} M_{\gamma^{\prime}}$, the Lagrangian term of their coupling to dark sector fermions and the decay width can be written as:

$$
\mathcal{L} \sim \sqrt{4 \pi \alpha^{\prime}} \bar{\chi} \gamma^{\mu} \chi \gamma_{\mu}^{\prime}
$$


and

$$
\Gamma_{\gamma^{\prime} \rightarrow \chi \chi}=\frac{1}{3} \alpha^{\prime} M_{\gamma^{\prime}} \sqrt{1-\frac{4 m_{\chi}^{2}}{M_{\gamma^{\prime}}^{2}}}\left(1+\frac{2 m_{\chi}^{2}}{M_{\gamma^{\prime}}^{2}}\right)
$$

where $\alpha^{\prime}$ is the coupling constant of dark fermions with their dark gauge boson. It is considered natural that $\alpha^{\prime} \gg \alpha \epsilon^{2}$ [57]. This generates an invisible decay portal for the dark photon to disintegrate into dark fermions escaping visible detection, and creates an opportunity for searches via missing mass or by looking at the scattering of $\chi(\mathrm{DM})$ with detector matter [58].

The basic physics of the vector portal assume several properties of the dark photon:

- The mixing parameter is conjectured to obey $\epsilon \ll 1$, while the dark coupling constant $\alpha^{\prime}$ can be comparable to the corresponding SM coupling $\alpha$.

- If the additional $\mathrm{U}(1)^{\prime}$ and $\mathrm{SM} \mathrm{U}(1)$ symmetries are unbroken, the mass of the $\mathrm{U}(1)^{\prime}$ mediator has a small mass $\left(m_{\gamma^{\prime}} \rightarrow 0\right)$ and the dark sector is inhabited by so-called "milli-charged particles" carrying $q_{\chi}=e \epsilon$ electric charge [55, 47].

- $\gamma^{\prime}$ conserves the following SM symmetries: parity, flavor, CP.

- $\gamma^{\prime}$ does not primarily interact with neutrinos, and interactions through darkphoton exchange are stronger than weak interactions.

\subsubsection{Anomalous Magnetic Moment}

The magnetic moment $\overrightarrow{\mathrm{M}}$ of a particle with spin $\overrightarrow{\mathrm{S}}$, charge $q$ and mass $m$ can be written as:

$$
\overrightarrow{\mathrm{M}}=g \frac{q}{2 m} \overrightarrow{\mathrm{S}}
$$

The value of $g$ can be defined very accurately from the Dirac equation to be equal to 2 at a tree-level calculation. The SM predicts a small deviation from this value for leptons due to quantum loop corrections and vacuum polarizations. This deviation is known as the anomalous magnetic moment, and it was confirmed by experimental measurements. It can be parametrized as $a=\frac{g-2}{2}$, where $a$ carries the contribution from QED $\left(a_{Q E D}\right)$, weak interactions $\left(a_{E W}\right)$, hadronic interactions $\left(a_{\text {hadrons }}\right)$. Potential extra interactions in the hidden sector could contribute to the anomalous magnetic moment as well. The summed contribution from the SM interactions for the $a$ parameter, $a_{S M}$, can be represented as:

$$
a_{S M}=a_{Q E D}+a_{E W}+a_{\text {hadrons }} .
$$




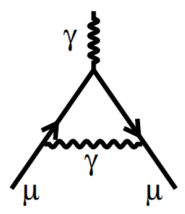

QED

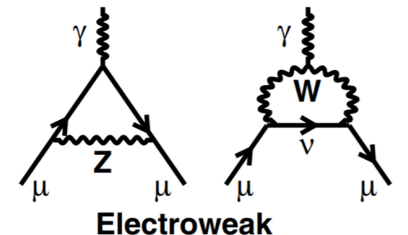

Electroweak

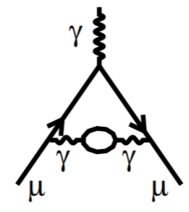

Hadron

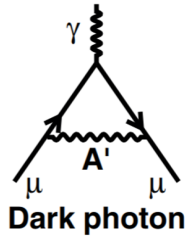

Figure 1.4.2.: Quantum corrections to the anomalous magnetic moment from various interactions [57].

For a leptophilic dark photon, the contribution $a_{\gamma^{\prime}}$ to $a$ can be expressed as [57]:

$$
a_{\gamma^{\prime}}^{\ell}=\frac{\alpha^{\prime}}{2 \pi} f\left(m_{\ell}, M_{\gamma^{\prime}}\right)
$$

where $\alpha^{\prime}=\alpha \epsilon^{2}$ is the coupling constant of the dark photon with lepton flavor $\ell$ and

$$
f\left(m_{\ell}, M_{\gamma^{\prime}}\right)=\int_{0}^{1} \frac{2 m_{\ell}^{2} z(1-z)^{2}}{m_{\ell}^{2}(1-z)^{2}+M_{\gamma^{\prime}}^{2} z},
$$

where $z$ is the momentum carried by the dark photon in lab frame. This $z$ factor is 1 if the mass of the dark photon is larger than the lepton mass $m_{\ell} \gg M_{\gamma^{\prime}}$ (eq.(4) in [59]), while for $m_{\ell} \ll M_{\gamma^{\prime}}$,

$$
f\left(m_{\ell}, M_{\gamma^{\prime}}\right)=\frac{2 m_{\ell}^{2}}{3 M_{\gamma^{\prime}}^{2}}
$$

The difference between the theoretical calculation of the anomalous magnetic moment and the experimentally measured value can be due to eq.(1.4.12) and can be explained using the contribution from eq.(1.4.11). Using this property the other way around, we can derive limits on the dark photon characteristics.

The most precise measurement of the electron anomalous magnetic moment [60] is related to the electromagnetic coupling constant in the SM through:

$$
\begin{aligned}
\frac{g_{e}}{2}= & +C_{2}\left(\frac{\alpha}{\pi}\right)+C_{4}\left(\frac{\alpha}{\pi}\right)^{2}+C_{6}\left(\frac{\alpha}{\pi}\right)^{3}+C_{8}\left(\frac{\alpha}{\pi}\right)^{4}+C_{10}\left(\frac{\alpha}{\pi}\right)^{5} \\
& +a_{Q E D}+a_{\text {hadrons }}+a_{\text {weak }}
\end{aligned}
$$

with the published experimental values of the fine structure constant and $a_{e}=\frac{g_{e}-2}{2}$

$$
\alpha^{-1}=137.035999084(51) \text {, }
$$


and

$$
a_{e}=0.00115965218073(28) \text {. }
$$

To extract possible new physics information from this measurement, a precise and independent calculation of $\alpha^{-1}$ and $a_{e}$ was performed for rubidium atoms [61]. The comparison of the $a_{e}$ parameter values obtained from the experiment (eq. (1.4.16)) and from the theoretical calculation, using the measurement of the fine structure constant [61], $\alpha^{-1}$, with the rubidium atoms (eq.(1.4.17)), favors the scenario with no dark photon contribution and can be used to exclude the dark photon hypothesis.

$$
\begin{aligned}
& \alpha^{-1}(\text { from } \mathrm{Rb} \text { atom })=137.035999044(90) \\
& a_{e}(\exp )-a_{e}(\text { from } \mathrm{Rb} \text { atom })=-1.09(0.83) \times 10^{-12}
\end{aligned}
$$

However, for the muon, the measured anomalous magnetic moment is estimated to be off from the theoretical prediction by more than $3 \sigma$. The resulting region in the $\epsilon$ vs $m_{\gamma^{\prime}}$ parameter space that could account for a $3.6 \sigma$ deviation due to a dark photon with the exclusion bounds at $1 \sigma, 2 \sigma, 3 \sigma$ confidence level coming from the electron magnetic moment is illustrated in Fig. 1.4.3.

\subsubsection{Motivation for Dark Photon Searches with an $e^{-}$Beam}

We consider in the following a minimalistic hidden sector model with light vector particles and one U(1) vector gauge boson as a mediator. The simplest way the SM can interact with such new boson, without the need to have SM particles charged under the additional symmetry, is kinetic mixing. As discussed in [47], two abelian gauge symmetries $U_{1}(1)$ and $U_{2}(1)$ can induce an effective charge at some scale without any charge (interaction) in the other. This implies that in a minimalistic extension to the SM with hidden fermions $\mathrm{f}_{12}$ charged under both $U_{1}(1)$ and $U_{2}(1)$, Feynman loop diagrams, as shown in Fig. 1.4.4, with aid of such virtual $f_{12}$ are creating a portal for the SM photon to interact with its hidden sector partner.

In the literature, dark photons are also known as heavy photons, secluded or hidden photons, paraphotons, and are typically denoted as $\gamma^{\prime}, A^{\prime}, \gamma_{D}$, and sometimes also $\mathrm{Z}^{\prime}$, following the hypercharge logic. Here it will be referred to as the dark photon, denoted $\mathrm{A}^{\prime}$ or $\gamma^{\prime}$.

The interaction dark photon mixing generates, respects parity and produces small dark charges and masses around the GeV scale. As discussed by Holdom [47], this is equivalent to an SM particle with an effective charge $\varepsilon e q_{i}$, where $\varepsilon=\frac{\varepsilon_{Y}}{\cos \left(\theta_{W}\right)}$, with $\theta_{W}$ the Weinberg-Salam mixing angle and $\varepsilon_{Y}$ is the coupling to hypercharge. 


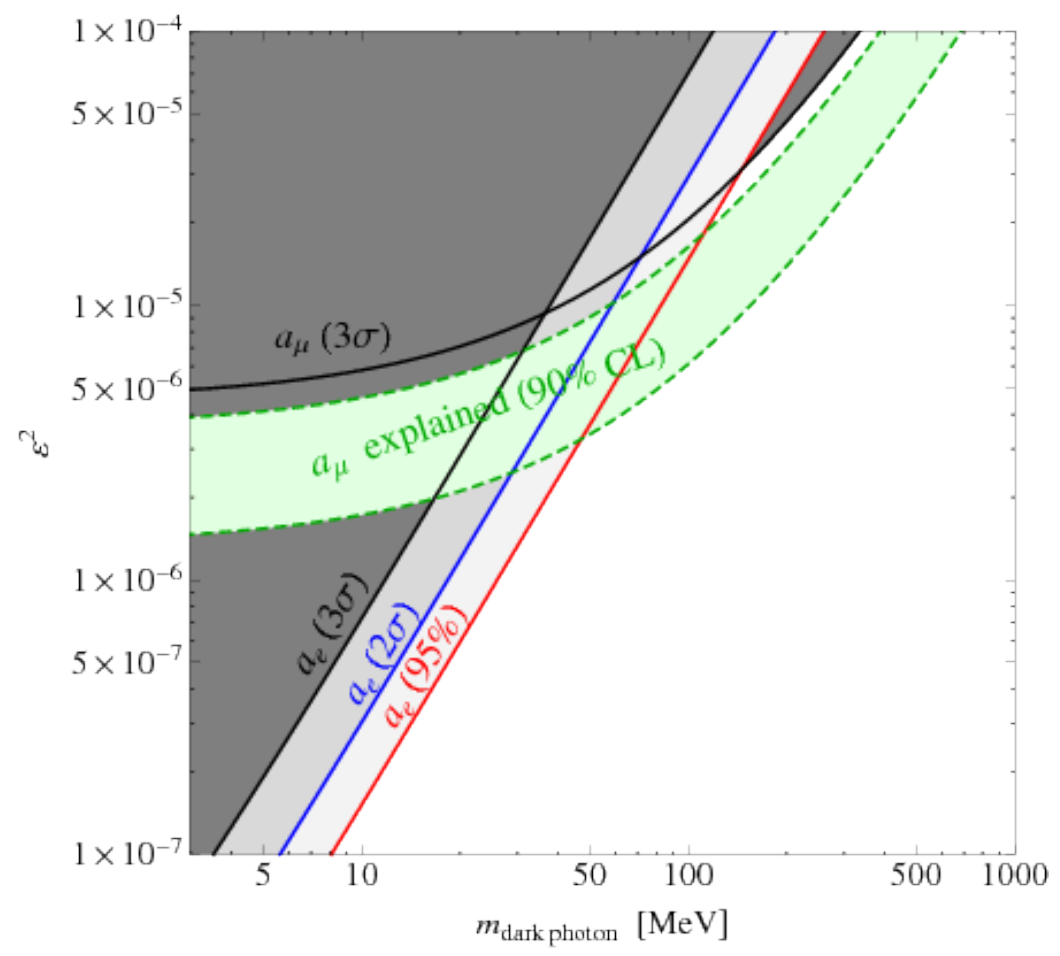

Figure 1.4.3.: Dark photon exclusion limits from the electron and muon anomalous magnetic moment [62]. The muon green band is the region where the $a_{\mu}$ magnetic moment $3.6 \sigma$ deviation would be fully explained by the existence of a dark photon.

Couplings through such quantum-mechanical corrections create a mixing factor of the order of [63]:

$$
\epsilon \sim 10^{-3}-10^{-2}
$$

relative to the electromagnetic coupling constant:

$$
\epsilon^{2} \sim \frac{\alpha^{\prime}}{\alpha} \sim 10^{-6}-10^{-4}
$$

The significance of these quantum effects does not depend on the mass of the hypothetical hidden fermions $m_{\chi}$, therefore it does not constrain the mass scale of $m_{\chi}$ except requiring it to be large enough to have escaped detection so far (i.e. above the weak scale). However, assuming a natural symmetry at high energies, e.g. introducing Grand Unification Theory with some gauge group, smaller couplings are expected. When symmetry is spontaneously broken at $M_{G U T} \approx 10^{16} \mathrm{GeV}$, the mixing is suppressed [63]:

$$
\epsilon_{G U T} \sim \frac{\alpha_{i}^{2}}{16 \pi^{2}} \ln \left(\frac{M_{G U T}}{M_{x}}\right) \sim 10^{-5}-10^{-3},
$$




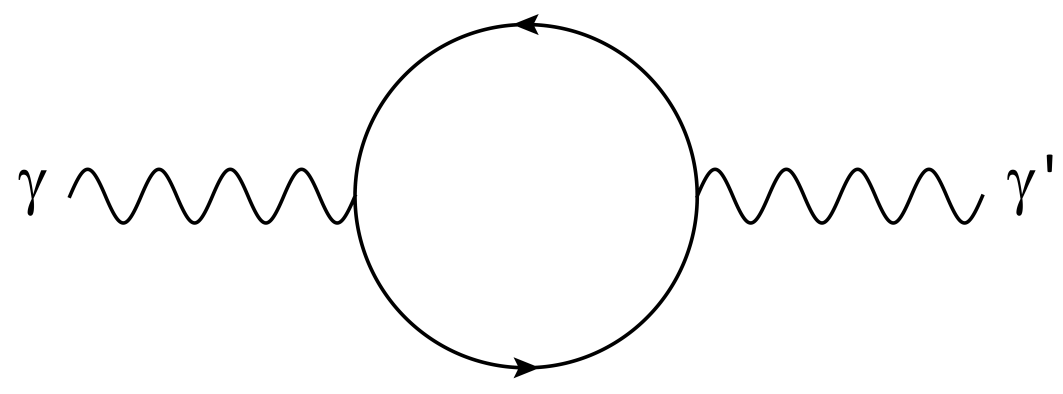

Figure 1.4.4.: Feynman diagram of a dark photon mixing with a light photon.

where $\alpha_{i}$ are gauge couplings. Then, the $\frac{\alpha^{\prime}}{\alpha}$ effective coupling is even more suppressed.

$$
\frac{\alpha^{\prime}}{\alpha} \sim 10^{-10}-10^{-6}
$$

This motivates the $\left(\epsilon\right.$ vs $\left.\mathrm{M}_{\gamma^{\prime}}\right)$ phase space range for a dark photon with masses $>50 \mathrm{MeV}$. Low energy supersymmetric models favor a mass range in the MeV$\mathrm{GeV}$ range [64]. The sub-GeV mass region is also motivated by [65], where $\mathrm{U}(1)^{\prime}$ breaking occurs similar to the hypercharge symmetry breaking of $\mathrm{U}(1)$ and the mass of the dark photon is generated from the same physics that gives mass to the weak interaction bosons.

Once the electroweak symmetry is broken via the Higgs mechanism, the Higgs field obtains a non-zero vacuum expectation value, so that this term generates a mass term for a hidden particle. Via kinematic mixing with the hypercharge photon, the $\mathrm{U}(1)^{\prime}$ symmetry breaking would then also generate a mass via the Higgs mechanism for the dark photon:

$$
M_{\gamma^{\prime}} \sim \sqrt{\epsilon} \sqrt{\frac{g_{D} g_{Y}}{g_{2}^{2}}} m_{W} \sim \mathrm{MeV}-\mathrm{GeV}
$$

where $g_{2}$ is the coupling of the Standard Model $\mathrm{SU}(2)_{\mathrm{L}}, m_{W}$ is the mass of the $\mathrm{W}$ boson and $g_{D}$ and $g_{Y}$ are the couplings of the hidden charge and hypercharge respectively. This estimate gives a mass range from $50 \mathrm{MeV}$ to $1 \mathrm{GeV}$ for $M_{\gamma^{\prime}}$ [63].

It is important to note that new matter particles charged under this new additional U(1) symmetry do not have a real electric "milli charge" and can interact with photon only through quantum loops.

\subsubsection{The Experimental Considerations for Dark Photon Search in HPS}

HPS is designed to detect the $\gamma^{\prime}$ production from a bremsstrahlung-like process using high-energy electrons scattering off a tungsten target with an expected cross section 


$$
\sigma_{\gamma^{\prime}} \sim 100 \mathrm{pb}\left(\frac{\epsilon}{10^{-4}}\right)^{2}\left(\frac{100}{M_{\gamma^{\prime}}} \mathrm{MeV}\right)^{2} .
$$

Heavy photons generated this way decay into electron-positron pairs, which can be detected. The dark photon will then appear as a narrow resonance peak in the $e^{+} e^{-}$ invariant mass spectrum on top of the QED processes. The Feynman diagrams of the dark photon production are illustrated in Fig. 1.4.5.

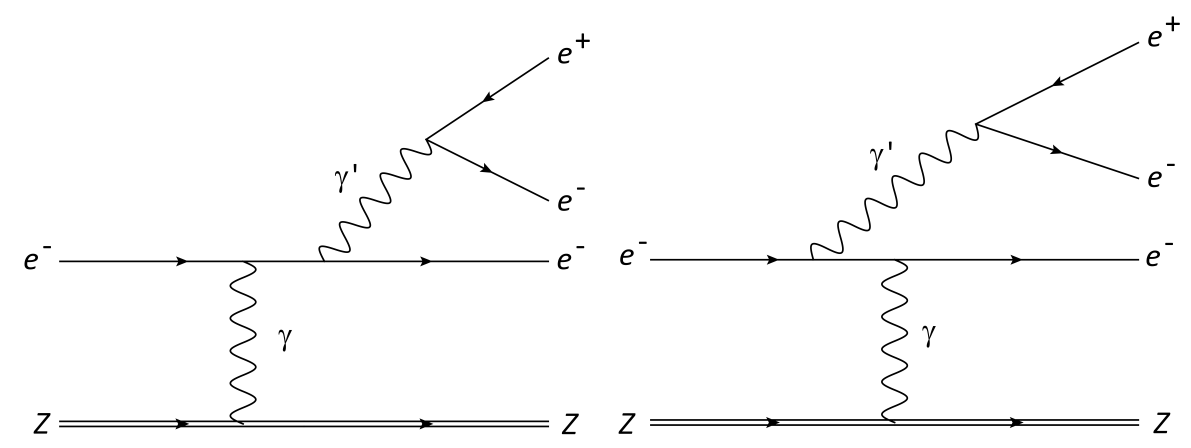

Figure 1.4.5.: Feynman diagram of dark-strahlung on a heavy target Z.

The differential cross section corresponding to eq.(1.4.24) is estimated by the WeizsackerWilliams approximation [66]:

$$
\frac{d \sigma}{d x d \cos \theta_{\gamma^{\prime}}} \approx \frac{8 Z^{3} \alpha^{3} \epsilon^{2} E_{0}^{2} x}{U^{2}} \tilde{\chi} \times\left[\left(1-x+\frac{x^{2}}{2}\right)-\frac{x(1-x) M_{\gamma^{\prime}}^{2} E_{0}^{2} x \theta_{\gamma^{\prime}}^{2}}{U^{2}}\right],
$$

where $E_{0}$ is the energy of the incoming electron, $E_{\gamma^{\prime}}=x E_{0}$ is the energy of the dark photon, $\mathrm{Z}$ is the atomic number of the target atom, $\theta_{\gamma^{\prime}}$ is the scattering angle of the $\gamma^{\prime}$ in the laboratory frame, and $\tilde{\chi}$ is the Weizsacker-Williams effective photon flux without its $Z^{2}$ overall factor. Furthermore, $\mathrm{U}\left(\mathrm{x}, \theta_{\gamma^{\prime}}\right)$ parametrizes the virtuality of the intermediate electron in the initial state bremsstrahlung, and is defined as:

$$
U\left(x, \theta_{\gamma^{\prime}}\right)=E_{0}^{2} x \theta_{\gamma^{\prime}}^{2}+\frac{(1-x) M_{\gamma^{\prime}}^{2}}{x}+m_{e}^{2} x .
$$

It is important to analyze the difference in cross section between ordinary photon breamsstrahlung and dark photon production, in order to optimize the experimental setting. The kinematics and rates of the a dark photon signal compared to its massless competitor are indeed different:

- The production rate of the $\gamma^{\prime}$ is controlled by the $\frac{\alpha^{3} \epsilon^{2}}{M_{\gamma^{\prime}}^{2}}$ term, and thus suppressed by $\sim \frac{\epsilon^{2} m_{e}^{2}}{M_{\gamma^{\prime}}^{2}}$ relative to the QED processes, which will be detailed in chapter 5 . 
- The energy of the $\gamma^{\prime}$ is peaked at the beam energy $\approx E_{0}$.

- The production angle of the $\gamma^{\prime}$ is favored at the angles where $\mathrm{U}\left(\mathrm{x}, \theta_{\gamma^{\prime}}\right) \lesssim 2 \mathrm{U}(\mathrm{x}, 0)$. This encourages a very forward $\gamma^{\prime}$ emission, as for larger angles the production drops very rapidly as $\theta_{\gamma^{\prime}}^{4}$. The $\gamma^{\prime}$ typically obtains the majority of the beam's energy and therefore cannot be too far off-axis as this would violate the conservation of the forward momentum in the production process:

$$
\theta_{\gamma^{\prime}, \max } \sim \max \left(\frac{\sqrt{M_{\gamma^{\prime}} m_{e}}}{E_{0}},\left(\frac{M_{\gamma^{\prime}}}{E_{0}}\right)^{\frac{3}{2}}\right) .
$$

Note that the opening angle of the decay products of the $\gamma^{\prime}$ can be larger than eq.(1.4.27), since the production angle is driven by the ratio of $M_{\gamma^{\prime}}$ and $E_{0}$, but the angle of the decay products is driven by the ratio of $M_{\gamma^{\prime}}$ and the mass of the decay products.

These properties of the $\gamma^{\prime}$ production kinematics are used for the design of the experiments for the heavy photon search. Small angles are experimentally challenging and require to have near beam plane detection. The issues induced by this will be discussed further, in chapter 3.

If the dark photon decays into SM particles and is not suppressed by decays to hidden states, it can travel macroscopic distances before disintegration. This would imply that a displaced vertex could be an observable signature in dark-photon search experiment. Neglecting phase-space corrections, the decay length of the $\gamma^{\prime}$ decaying into SM particles can be estimated as [66]

$$
l_{0} \equiv \gamma c \tau \simeq \frac{3 E_{\gamma^{\prime}}}{N_{e f f} M_{\gamma^{\prime}}^{2} \alpha \epsilon^{2}} \simeq \frac{0.8 \mathrm{~cm}}{N_{e f f}}\left(\frac{E_{0}}{10 \mathrm{GeV}}\right)\left(\frac{10^{-4}}{\epsilon}\right)^{2}\left(\frac{100 \mathrm{MeV}}{M_{\gamma^{\prime}}}\right)^{2},
$$

where $N_{\text {eff }}$ is the number of available decay channels. For heavy photons mixing only with leptons, $N_{\text {eff }}=1$, if $M_{\gamma^{\prime}}<2 m_{\mu}$. Then only the $\gamma^{\prime} \rightarrow e^{-} e^{+}$decay is possible. For higher mass, not only muons are possible, but also hadrons, making $N_{\text {eff }}$, therefore, increase very fast for large masses.

For a thin target with radiation length $X_{0}\left[\frac{\mathrm{g}}{\mathrm{cm}^{2}}\right]$ much bigger than the target thickness $T$, the total number of dark photons produced in the scattering of $N_{e}$ electrons off the target can be calculated as [66]:

$$
N \sim N_{e} \frac{N_{A} X_{0}}{A} T \frac{Z^{2} \alpha^{2} \epsilon^{2}}{M_{\gamma^{\prime}}^{2}} \tilde{\chi} \sim N_{e} C T \frac{\epsilon^{2} m_{e}^{2}}{M_{\gamma^{\prime}}^{2}} .
$$

Here $N_{A} \simeq 6 \times 10^{23}\left[\right.$ mole $\left.^{-1}\right]$ is the number of Avogadro, $A\left[\frac{g}{\text { mole }}\right]$ is the atomic mass of the target and $C \approx 5$ a factor depending logarithmically on the choice of the target nucleus and on the heavy photon's mass. The charge of the incident electrons, then, is given by:

$$
\frac{N}{C} \sim 10^{6} \frac{T}{0.1}\left(\frac{\epsilon}{10^{-4}}\right)^{2}\left(\frac{100 \mathrm{MeV}}{M_{\gamma^{\prime}}}\right)^{2} .
$$




\subsection{Conclusion}

In conclusion, the main points of this chapter can be summarized as follows:

- Modern physics describes the observable nature better than ever throughout its history. However, more experimental knowledge also reveals more problems in the current theoretical models, requiring extensions to the existing framework.

- The kinetic mixing mechanism allows for SM particles to interact with their hidden partners, opening portals to unexplored domains of physics.

- A dark photon is well motivated by many theories, arising naturally from beyond Standard Model physics in most conceptually minimalistic models.

- Dark photons can be produced in dark-strahlung by analog of regular photon production in bremsstrahlung processes. High intensity electron beams scattering off a target can induce such processes along with QED processes, followed by a decay to leptons.

- Exploiting the characteristic kinematical properties of such decay, the HPS experiment is searching for a dark photon resonance in the electron-positron invariant mass spectrum. In case of on-shell dark photon production, a displaced vertex observation provides a secondary signature for the HPS experiment. 



\section{Dark Photon Hunt in the World}

\subsection{Introduction}

The properties of the dark photon are subject to strong constraints, from tests in beam-dump experiments, fixed target experiments, cosmological observations through possible connections with the SM, and precision measurements of the magnetic anomalous moment introduced earlier.

The dark photon search motivates many experiments in the world using various detection techniques. They can be categorized into two classes, depending on whether the hidden photon production is visible or invisible, meaning whether the decay products are dark sector particles (invisible for direct detection) or not.

Visible decay experiment searches rely on the presumption that the $\gamma^{\prime}$, as the lightest hidden sector particle, decays to an SM pair.

\subsection{Beam-Dump Experiments}

Beam-dump experiments probe dark photon production through bremsstrahlung of high-intensity beams dumped on thick targets. As the mixing parameter, $\epsilon$, gets smaller, the lifetime of the dark photon becomes longer and displaced vertex searches are possible. The prospective dark photon decays into a lepton pair and it can be detected after sufficient shielding to absorb the SM particles leaving only long-lived, weakly coupled particles. These experiments are looking for a discrepancy from the expected background as a sign of new physics. Depending on the beam, energy, target, shielding length, and distance to the detectors, different $\gamma^{\prime}$ production mechanisms can be addressed. Electron and proton beams are used to probe leptophilic or baryonophilic $\gamma^{\prime}$ decays.

Electron beam-dump experiments (E141 (SLAC), E137 (SLAC), E774 (FermiLab), KEK-Japan, Orsay) have been searching for light metastable (pseudo)scalar states, with similar designs, as illustrated in Fig. 2.2.1. They cover a phase space for small $\gamma^{\prime}$ masses and down to very weak couplings.

$\operatorname{KEK}(1986)$ This experiment used a $2.5 \mathrm{GeV}$ electron beam with a total of $1.69 \times$ $10^{17}$ electrons interacting with a tungsten target, 2.2 meters of shielding 


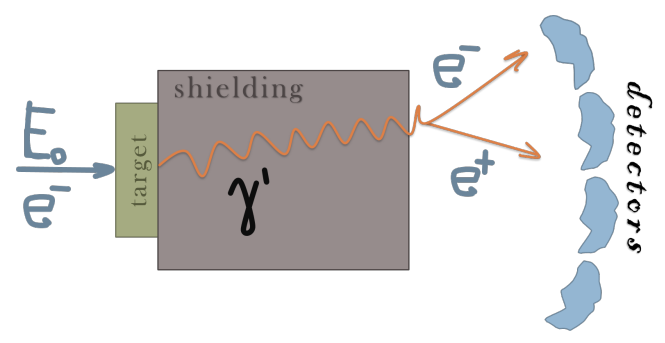

Figure 2.2.1.: Schematic drawing of a typical electron beam-dump experiment searching for a dark photon in the electron-positron decay channel.

made of iron, lead and plastic layers, multiwire proportional chambers, scintillator and Cherenkov counters [67]. No signal was observed.

E141(1987) A $9 \mathrm{GeV}$ electron beam scattering off a $12 \mathrm{~cm}$ tungsten target with a spectrometer at a distance of 35 meters. The experiment was searching for short living axions. With a total of $2 \times 10^{15}$ electrons on target, they obtained a 95\% CL upper limit [68].

E137(1988) A $20 \mathrm{GeV}$ electron beam dumped on an aluminum target with 179 meters of hill, which was used as shielding, and a 204 meters valley, used as a decay region, and an electromagnetic calorimeter to detect the signal. The experiment did not detect any axions or axion-like signals after a total of $1.86 \times 10^{20}$ electrons interacted with the target [69].

Orsay(1989) A $1.6 \mathrm{GeV}$ electron beam, a 2 meters long tungsten target, and a $10 \mathrm{~cm}$ wide decay region. Scintillator and lead-glass Cherenkov counters were used to search for a light Higgs boson in electron-positron pair decay above $0.75 \mathrm{GeV}$. The results did not show any significant signal [70].

E774(1991) A $275 \mathrm{GeV}$ electron beam, a $30 \mathrm{~cm}$ thick tungsten target, a 2-meters long decay region and scintillators used to detect neutral bosons decaying into electron-positron pairs. After a total of $5.2 \times 10^{9}$ incoming electron interactions, no signal was observed[71].

These experiments were reviewed in the context of the dark photon search and the exclusion limits on the mass and kinetic mixing parameter are shown in Fig. 2.2.2.

Proton beam dump experiments can probe dark photon production both by protons or leptons in bremsstrahlung-like processes and in various secondary mesonic decays, e.g. $\pi^{0} \rightarrow \gamma A^{\prime}$, opening an opportunity to study baryonophilic dark photon channels.

CHARM(1986) A $400 \mathrm{GeV}$ proton beam at CERN's SpS was scattering off a copper target [73]. The detector was set up $480 \mathrm{~m}$ from the target with a decay region of $3 \times 3 \times 35 \mathrm{~m}^{3}$, inside which three trackers and calorimeters were located. The experiment was searching for heavy neutrino decays $\nu_{h} \rightarrow \nu e^{+} e^{-}$within a $10 \mathrm{MeV}$ to $1.8 \mathrm{GeV}$ mass interval, where $\nu_{h}$ was expected to be produced in mesonic decays $(\pi, K, D)$. The dark-photon signal would be an excess of electron-positron pairs above the expected 


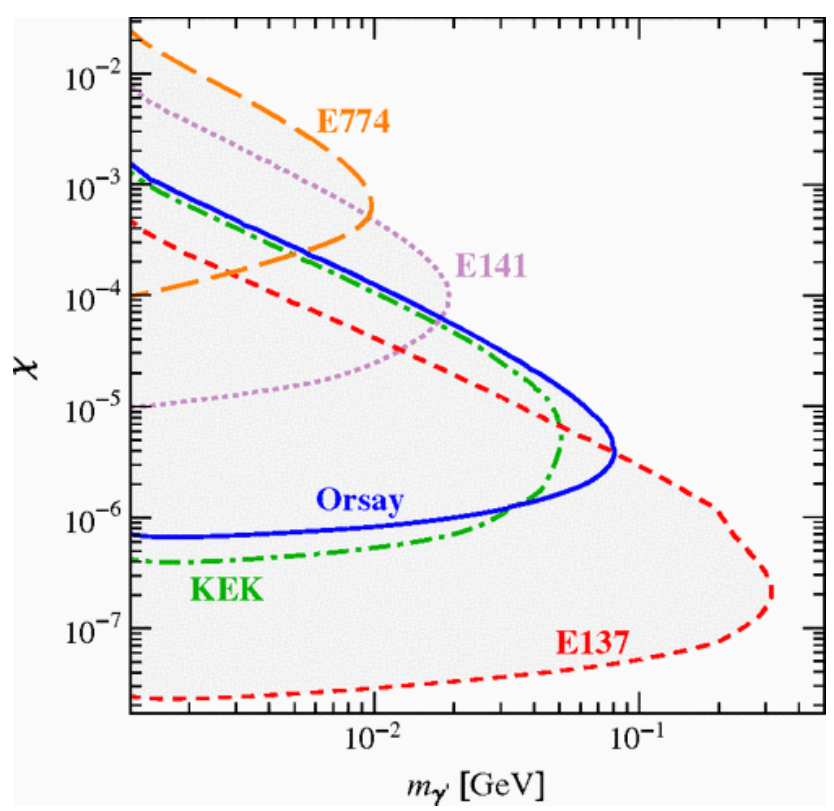

Figure 2.2.2.: Limits on dark photon masses in electron beam dump experiments [72].

background. The experiment did not observe any $\gamma^{\prime}$ events after a total of $2.4 \times 10^{18}$ proton-target interactions [74].

PS191(1986) A $19.2 \mathrm{GeV}$ proton beam (CERN, PS) was interacting with a Beryllium target with detectors at a distance of 128 meters behind the target. The experiment was designed to search for heavy neutrinos in these decay channels: $\nu_{h} \rightarrow e^{ \pm} \pi^{\mp}, \nu_{h} \rightarrow e^{+} e^{-} \nu$. In total $0.86 \times 10^{19}$ protons were shot at the target, but no heavy neutrino signal was observed [75].

$\nu$-Cal(1989) A $70 \mathrm{GeV}$ proton beam was dumped onto an iron target searching for axions and light Higgs bosons (at U70). The results were revisited for a search of dark gauge bosons in $\pi^{0}$ decay and proton bremsstrahlung [76].

NOMAD This was another experiment at CERN using the SPS and looking for $\nu_{\mu} \rightarrow \nu_{\tau}$ neutrino oscillations with a neutrino beam. The data were reconsidered for dark photon models and exclusion limits were obtained [57].

The exclusion limits obtained from all of these experiments are summarized in Fig. 2.2.3. It is important to note that if the coupling of the dark photon to the $\mathrm{SM}$ is different for leptons and baryons, the limits from proton beam-dump experiments are not valid for leptophilic hidden photons and, correspondingly, the results of electron beam-dump experiments are not valid for baryophilic hidden photons. 


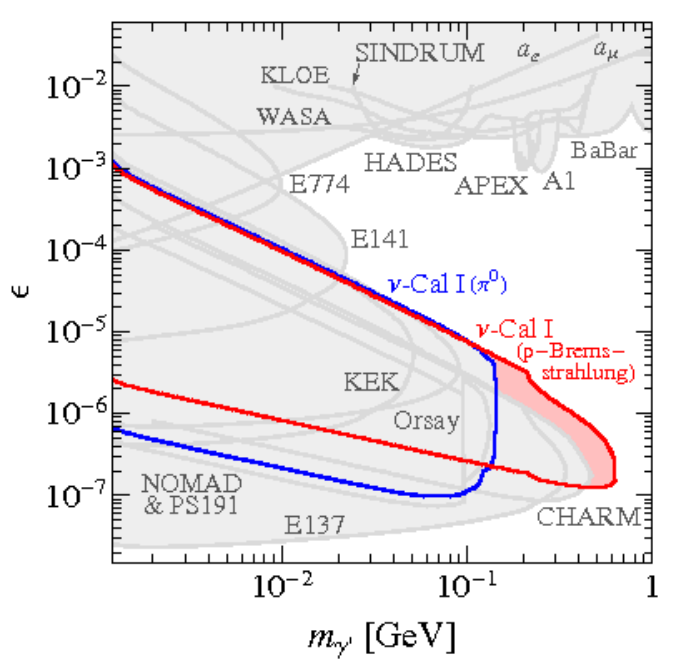

Figure 2.2.3.: Limits on dark photon mass and kinetic mixing from proton beam dump experiments [76].

\subsection{Collider Experiments}

Collider experiments were also performed for dark photon searches, providing a coverage for larger masses and kinetic mixing parameters. Some of these experiments are briefly summarized below (KLOE, BaBar and BESIII).

$\operatorname{KLOE}(2002,2004-2005)$ is a detector at INFN coupled with an electron-positron collider running at energies around the $\phi$ meson mass $(\sim 1.019 \mathrm{GeV})$. The dark photon search was done through the $\phi \rightarrow \eta \gamma^{\prime}, \gamma^{\prime} \rightarrow e^{+} e^{-}$ and $\gamma^{\prime} \rightarrow \mu^{+} \mu^{-}$channels, where the $\eta \rightarrow \pi^{+} \pi^{-} \pi^{0}$ and $\eta \rightarrow \pi^{0} \pi^{0} \pi^{0}$ decays were used to tag the $\eta$ mesons. Data samples of $1.5 \mathrm{fb}^{-1}$ and $1.7 \mathrm{fb}^{-1}$ were collected in 2004-2005 and 13000 events were selected by $\eta \rightarrow \pi^{+} \pi^{-} \pi^{0}$ tagging and 31000 by $\eta \rightarrow \pi^{0} \pi^{0} \pi^{0}$ tagging. For a dark gauge boson search through its dimuon decay mode, data were taken in 2002 , and about $239.3 \mathrm{pb}^{-1}$ was used. Analyses are carried out using bump hunt techniques on the invariant mass spectrum of $m_{e^{+} e^{-}}$and $m_{\mu^{+} \mu^{-}}$. The results are summarized in Fig. 2.3.1 where a composite $90 \%$ exclusion limit is set with confidence levels $(C L)$ calculations for the $\phi \rightarrow \eta e^{+} e^{-}\left(K L O E_{1}\right), e^{+} e^{-} \rightarrow \mu^{+} \mu^{-} \gamma\left(K L O E_{2}\right)$ and $e^{+} e^{-} \rightarrow e^{+} e^{-} \gamma$ $\left(K L O E_{3}\right)$ channels. The gap between $K L O E_{1}$ and $K L O E_{2}$ is due to the $\rho$ meson at $770 \mathrm{MeV}$, around which the experiment is not sensitive to a signal in dimuon decay.

BABAR at SLAC has searched for a dielectron decay of a dark photon in the processes $e+e^{-} \rightarrow \gamma^{\prime} \gamma, \gamma^{\prime} \rightarrow e^{+} e^{-}$and $\gamma^{\prime} \rightarrow \mu^{+} \mu^{-}$. The data sample of $514 \mathrm{fb}^{-1}$ was collected from $\Upsilon(2 \mathrm{~S}), \Upsilon(3 \mathrm{~S}), \Upsilon(4 \mathrm{~S})$ resonances and a bump- 


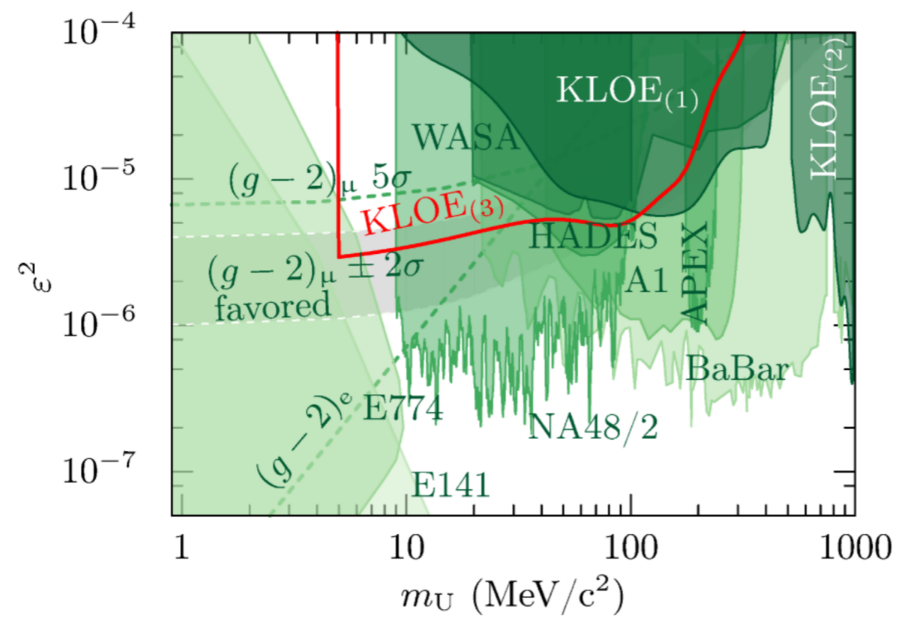

Figure 2.3.1.: Limits on dark photon mass and kinetic mixing from KLOE [77].

hunt scan was performed in the range $0.02<m_{\gamma^{\prime}\left(e^{-} e^{+}\right)}<10.2 \mathrm{GeV}$ for electron-positron decays and $0.0212<m_{\gamma^{\prime}\left(\mu^{-} \mu^{+}\right)}<10.2 \mathrm{GeV}$ for muon pair decay channels [78]. About $53 \mathrm{fb}^{-1}$ of data were collected for the invisible decay channel $e+e^{-} \rightarrow \gamma^{\prime} \gamma, \gamma^{\prime} \rightarrow$ invisible [79] up to the $8 \mathrm{GeV}$ mass range. Exclusion limits at a $90 \%$ confidence level were set, see Fig. 2.3.2. Thus, the dark photon mass region motivated by the muon $\left(g_{\mu}-2\right)$ anomaly has been ruled out for $\gamma^{\prime}$ visible and invisible decay mode.
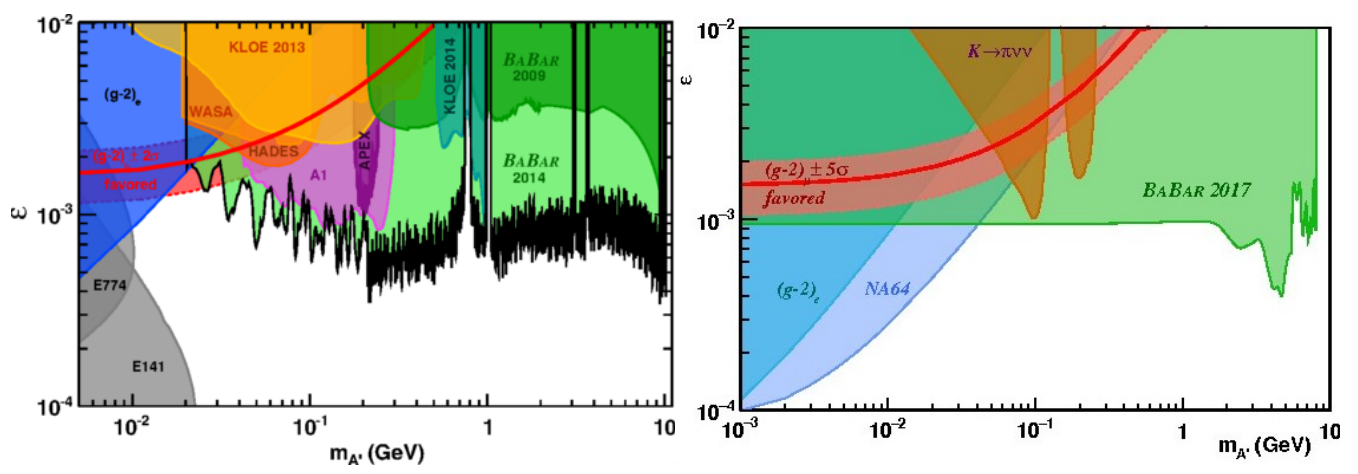

Figure 2.3.2.: Limits on dark photon mass and kinetic mixing from BaBar in the visible (left) [78] and the invisible (right) [79] decay searches.

BESIII(2008) is a detector at the BEPCII (China) collider. It was used for a dark photon search in the initial state radiation reactions $e^{+} e^{-} \rightarrow e^{+} e^{-} \gamma_{I S R}$ and $e^{+} e^{-} \rightarrow \mu^{+} \mu^{-} \gamma_{I S R}$, looking for a peak in the invariant mass of $e^{+} e^{-}$or $\mu^{+} \mu^{-}$as a $\gamma^{\prime}$ signature. From $2.93 \mathrm{fb}^{-1}$ of data, no signal was 
observed and a $90 \%$ confidence level upper limit was set in the mass range $1.5-3.4 \mathrm{GeV} / \mathrm{c}^{2}[80]$.

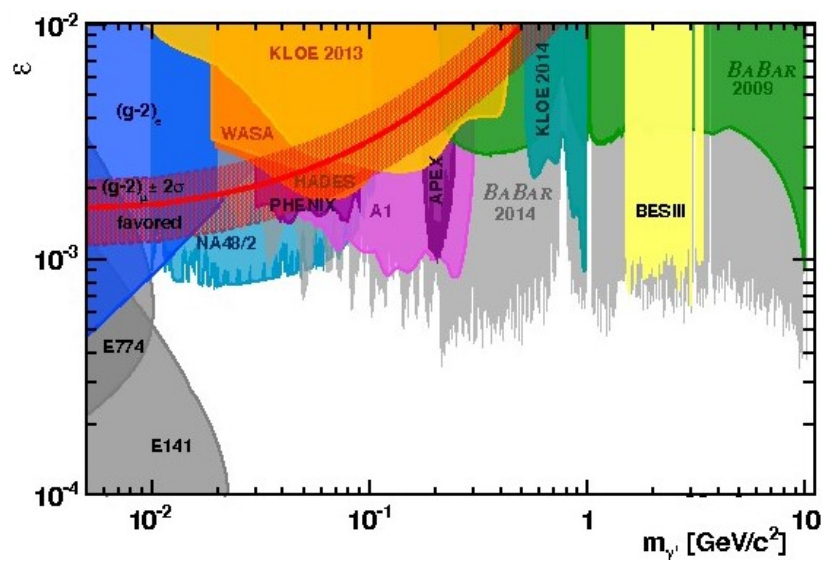

Figure 2.3.3.: Limits on dark photon mass and kinetic mixing from BESIII [81] and others.

\subsection{Fixed Target Experiments}

Fixed target experiments are an excellent tool for dark photon searches in the subGeV-GeV mass regions. Here we review APEX and HPS at JLab, HADES at GSI, NA48/2 at CERN SPS and A1 at MAMI:

A1 is an experiment based at the Mainz Microtron, which is using an unpolarized electron beam with energies ranging from $180 \mathrm{MeV}$ to $855 \mathrm{MeV}$, impinging upon a target from an assembly of tantalum foils. The dark photon to lepton pair decay channel was studied and an upper limit was set at a $90 \%$ confidence level, using the Feldman-Cousins algorithm, in the $\gamma^{\prime}$ mass range from $40 \mathrm{MeV} / \mathrm{c}^{2}$ to $300 \mathrm{MeV} / \mathrm{c}^{2}$, as shown in Fig. 2.4.1.

HADES (High-Acceptance DiElectron Spectrometer) is an experiment at the GSI in Darmstadt. A $3.5 \mathrm{GeV}$ proton beam interacting with a liquid hydrogen or solid niobium target, as well as $1.756 \mathrm{GeV}$ Ar and KCL targets are used to study dark-photon production in the $\gamma^{\prime} \rightarrow e^{+} e^{-}$decay mode. The spectrum of the $e^{+} e^{-}$invariant mass was reconstructed from $\pi^{0}$, $\eta$ mesons and $\Delta$ resonance decays and was studied with a bump hunt search. Narrow resonance search-peaks were modeled as Gaussians and the background as a 5th order polynomial, where the floating mass and width of the peak were fixed for each fit using MC simulation data. The analysis did not observe any resonance and an upper limit at 90\% CL 


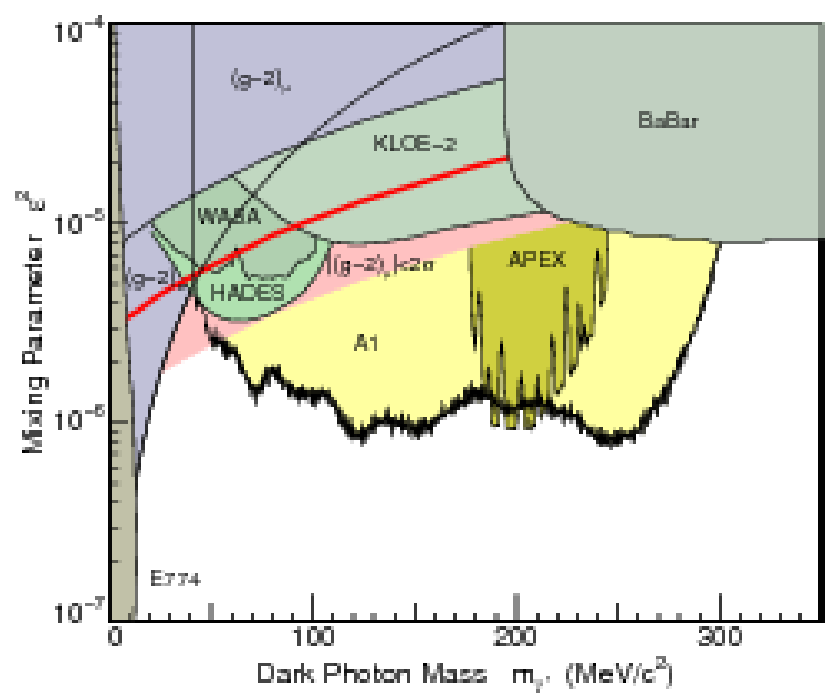

Figure 2.4.1.: Limits on dark photon mass and kinetic mixing from A1 MAMI [82].

was set, see Fig. 2.4.2.

NA48/2(2003-2004) at CERN searched for an immediate dark photon decay in the process $\pi^{0} \rightarrow \gamma^{\prime} \gamma, \gamma^{\prime} \rightarrow \gamma e^{+} e^{-}$with a high intensity beam of kaons. From $1.69 \times 10^{7}$ reconstructed $\pi^{0} \rightarrow \gamma e^{+} e^{-}$events, an analysis extracted exclusion limits at the $90 \%$ C.L. in the mass range $9-70 \mathrm{MeV} / c^{2}$. The experiment excluded at that point the phase space for a dark photon as an explanation for the $\left(g_{\mu}-2\right)$-anomaly, assuming a coupling to quarks and dominant decays to SM fermions.

APEX is an experiment at JLab in Hall A, searching for dark photons in the dielectron decay channel. A high-intensity electron beam scatters off a tantalum target, and the decay products are detected in two-arm high-resolution spectrometers registering electrons and positrons carrying about half the beam's energy. The test run with a $2.26 \mathrm{GeV}$ electron beam and a current of $150 \mu \mathrm{A}$ was performed in 2010. A binned profile likelihood with a $0.05 \mathrm{MeV}$ bin size was fit in the mass range of $175-250 \mathrm{MeV}$. No signal was observed and a $90 \%$ confidence level upper limit was calculated in Fig. 2.4.4. The full run plans are for 200 times more data and a larger mass coverage through various beam settings.

HPS(2014-...) at JLab, the subject of this thesis, is a fixed target experiment as well. There was a test run in 2012, which was used to confirm the feasibility of the experiment, the trigger rates and occupancies. The HPS test-run apparatus, the readout electronics and the performance are discussed in 


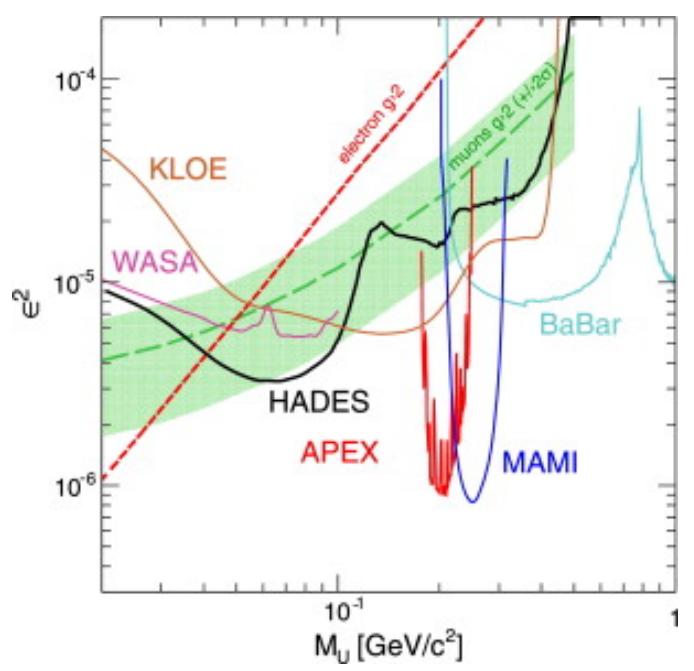

Figure 2.4.2.: Limits on dark-photon mass and kinetic mixing reported by HADES [83], compared with MAMI/A1, APEX, BaBar, WASA, KLOE and (g-2) measurements.

[85]. The experimental reach was estimated from simulations in [63]. In 2015, HPS had an engineering run using different configurations. The data from this run are partially analyzed and the results will ultimately be published. In figure Fig. 2.4.5, the experimental reach estimated from the simulations at three different beam energies is presented, showing the coverage for resonance searches and displaced-vertex searches.

\subsection{Searches in Dark Photon Invisible Decays}

Invisible decay searches are looking for a signal where the hidden photon decays to non-SM particles, assuming there is at least one hidden sector state $\chi$ lighter than the dark photon. The experiments make use of missing mass techniques or massive detectors to register dark photon decay products in scatterings. The common design of these experiments is based on the processes where the beam particle produces a $\gamma^{\prime}$ in a bremsstrahlung-like process and the dark photon subsequently decays into a dark matter $\chi$ pair. If $M_{\gamma^{\prime}}<2 m_{\chi}$, the production of dark particles is best investigated in fixed target experiments with an electron beam, where a radiative off-shell $\gamma^{\prime}$ is produced with a yield $\sim \frac{\alpha^{\prime} \epsilon^{2}}{m_{\chi}^{2}}$ [57]. In the case $M_{\gamma^{\prime}}>2 m_{\chi}$, there is secondary beam of dark matter particles with a rate $\sim \frac{\epsilon^{2}}{M_{\gamma^{\prime}}^{2}}[57]$. The $\chi$ interaction with the detector matter through $\gamma^{\prime}$ exchange occurs with a rate proportional to $\frac{\alpha^{\prime} \epsilon^{2}}{2 M_{\gamma^{\prime}}^{2}}$ resulting in a suppression factor for on-shell dark photon production $\sim \frac{\alpha^{\prime} \epsilon^{4}}{M_{\gamma^{\prime}}^{4}}$. Therefore, to be sensitive to dark photon production, the experiments are designed 


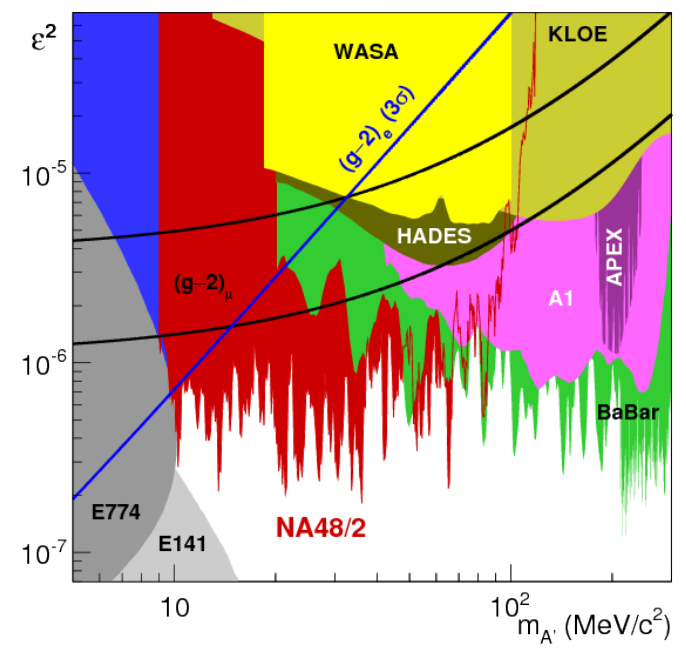

Figure 2.4.3.: Limits on dark photon mass and kinetic mixing from NA48/2 [81] and others.

with an intensive beam dumping on a high Z target. The E137 and LSND(19931998) experiments were revisited recently and, for several $\alpha^{\prime}$ values, exclusion limits on dark photon production were calculated [87].

Other experiments of dark photon invisible decays rely on direct detection of $\gamma^{\prime} \mathrm{s}$ in kinematically constrained final states through missing mass. In the case of an electron beam, the recoil nucleus carries the smallest part of the interaction energy most of it is transferred to the dark photon, which then decays into a $\chi \chi$, leaving a small energy incident electron measured in the calorimeter as a signature. The dominant background for this processes are nuclear reactions with neutrino production, bremsstrahlung photons and scattered electrons showering in an electromagnetic calorimeter. Number of experiments are carried out and planned for future runs (VEPP-3 [88] at BINP, NA62 [89] at CERN). Current exclusion limits from missing energy studies in BaBar [79], N64 [90], E949 [91] and E787 experiments at Brookhaven National Laboratory, as well as constraints from muon anomalous magnetic momentum precision measurements, are displayed in Fig. 2.5.1.

More experiments searching for a dark photon are expected in the upcoming years using different techniques and strategies. Cornell's Wilson Laboratory has proposed a $\gamma^{\prime}$ search through invisible decay with a positron beam, impinging on a beryllium target [92]. A similar experiment is planned at Frascati (LNF) with a 550 $\mathrm{MeV}$ positron beam and thin target [93], VEPP-3 [88] at BINP with a positron beam and a gas hydrogen target and NA62 [89] and P348 [56] at CERN are also looking for dark photon through invisible decays. The Search for Hidden Particles collaboration (SHiP) has proposed a proton beam dump experiment designed for the detection of light dark matter particles with masses up to $\mathrm{O}(10) \mathrm{GeV}$ both through bremsstrahlung and QCD production processes [94]. Belle II at KEK is an experiment at the SuperKEKB collider in Tsukuba in Japan, which is searching for 


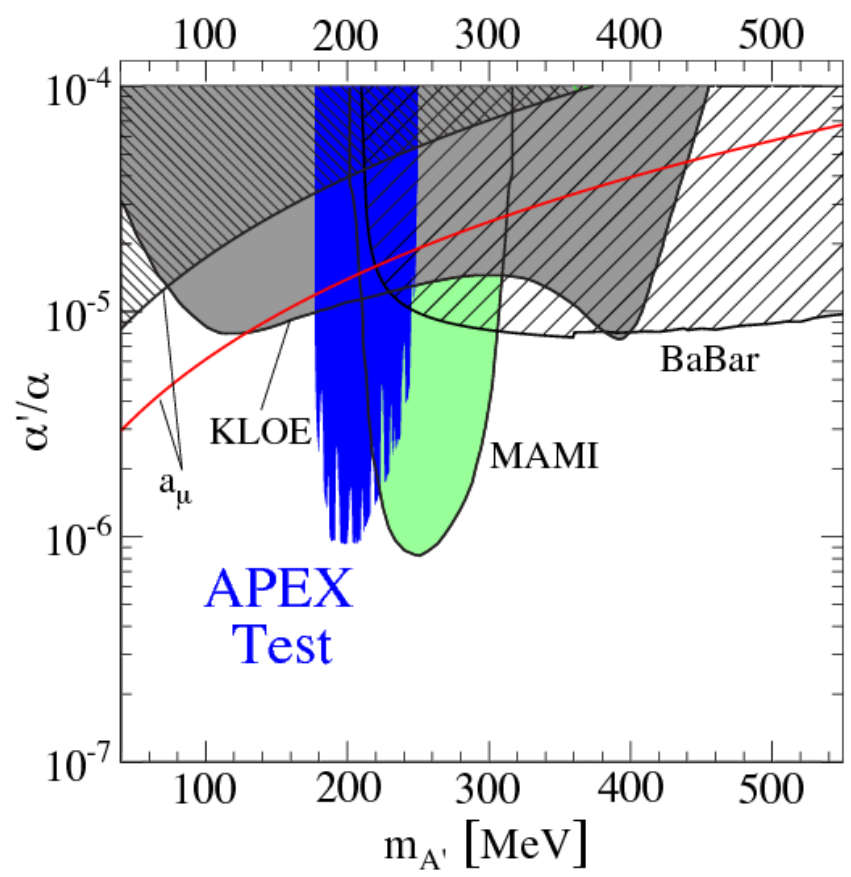

Figure 2.4.4.: Limits on dark photon mass and kinetic mixing from APEX [84] and others.

dark photons both in invisible decays and in the dimuon invariant mass spectrum [95]. There is another beam dump experiment proposed at JLab for dark photon detection through a rare interaction of its decay products with detector matter. The DarkLight experiment [96] is proposing to measure the $\gamma^{\prime} \rightarrow e^{+} e^{-}$decay in electron-proton scattering with a high intensity electron beam of $100 \mathrm{MeV}$, an hydrogen target and full detection of the residuals. This experiment is planned to be optimized for a $\gamma^{\prime}$ invisible decay search.

Rare muon decays have been investigated as a dark photon production channel as well, and it was shown [97] that for the lepton flavor violating decay $\mu^{+} \rightarrow e^{+} e^{-} e^{+}$, the $\gamma^{\prime}$ can be produced by an off-shell $W^{+}$from both the muon or the electron. This measurement is planned by the Mu3e experiment [97] at the Paul Scherrer Institute in Switzerland.

\subsection{Higgs portal searches}

Production of dark photons in models with a dark Higgs are also explored by several collider experiments in the channels $e^{+} e^{-} \rightarrow \gamma^{\prime} h^{\prime}, h^{\prime} \rightarrow \gamma^{\prime} \gamma^{\prime}$ (BaBar [98]), $e^{+} e^{-} \rightarrow$ $\gamma^{\prime} h^{\prime}$. Here the $\gamma^{\prime}$ decays visibly, whilst the dark Higgs can be found by looking for its missing energy (KLOE2 [99]). The Higgs portal has caught attention at Belle as well. The experiment has exploited several exclusive and inclusive decay reactions 


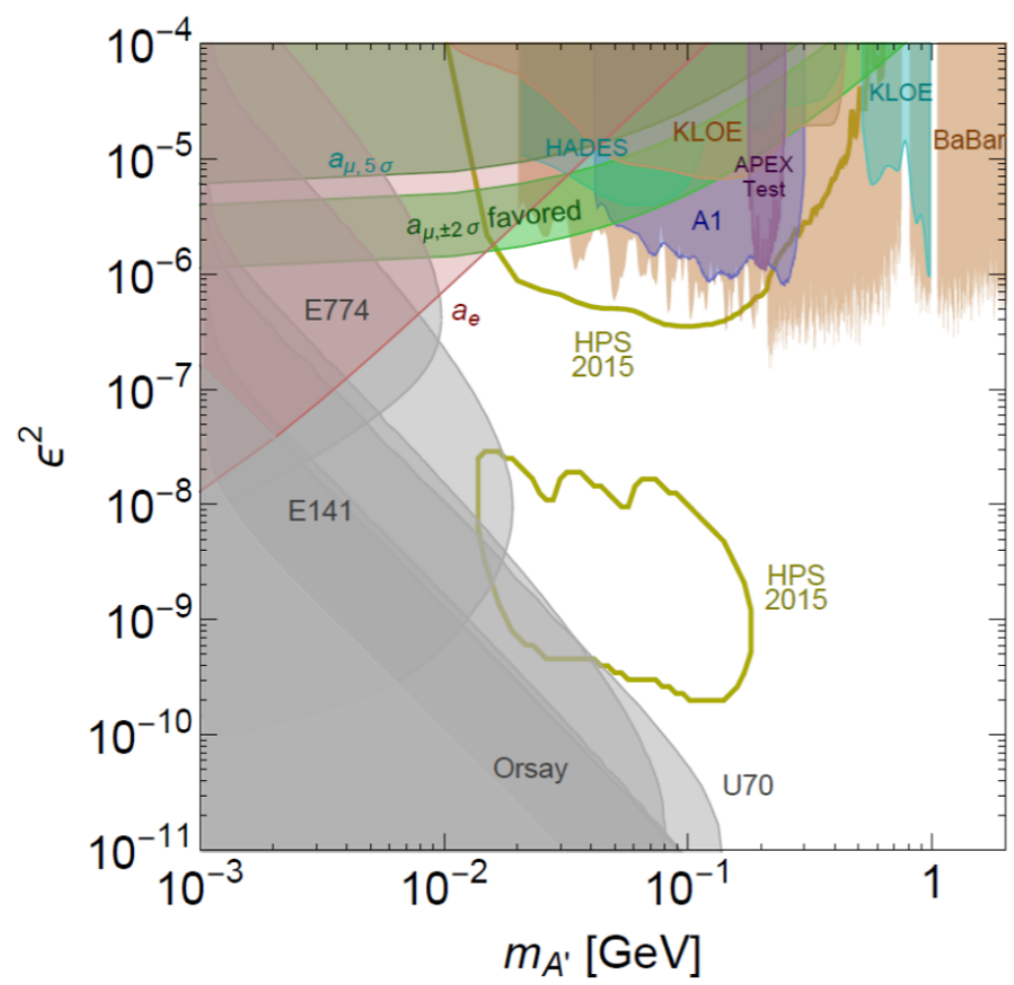

Figure 2.4.5.: Limits on dark photon mass and kinetic mixing anticipated by HPS. The regions within HPS lines show the experimental reach for bump hunt (open line for $\epsilon^{2} \in\left[10^{-7}, 10^{-4}\right]$ ) and displaced vertex search (closed line for $\left.\epsilon^{2} \in\left[10^{-10}, 10^{-7}\right]\right)$ for all energies combined $(1.1 \mathrm{GeV}, 2.2 \mathrm{GeV}, 6.6 \mathrm{GeV})$ energies combined [86].

to extract both dark photons and dark Higgs production information [100]. All the results from these experiments are coherent with the expected background. Future upgrades are promising more sensitivity and better measurements.

The ATLAS collaboration has searched for a dark photon in displaced jets of light leptons and hadrons, as shown in Fig. 2.6.1, with the center of mass energy $\sqrt{s}=$ $8 \mathrm{TeV}$. A data sample of size $20.3 \mathrm{fb}^{-1}$ was obtained and analyzed in 2012 and the results were interpreted in terms of a dark photon vector portal when setting exclusion limits, which are shown in Fig. 2.6.2. More studies at higher $\sqrt{s}=14 \mathrm{TeV}$ center of mass energy are being carried out in [101].

The CMS collaboration also studied $\gamma^{\prime}$ production, in this case decaying to a dimuon pair jet [103]. Using $20.7 \mathrm{fb}^{-1}$ of data from proton-proton collisions at a center of mass energy $\sqrt{s}=8 \mathrm{TeV}$, exclusion limits were set at a $95 \%$ confidence level in the $\gamma^{\prime}$ parameter space considering two different model types: non-minimalistic supersymmetric models and hidden sector models with a relatively long-lived light gauge boson. This experiment has covered a large portion of the dark photon mass 


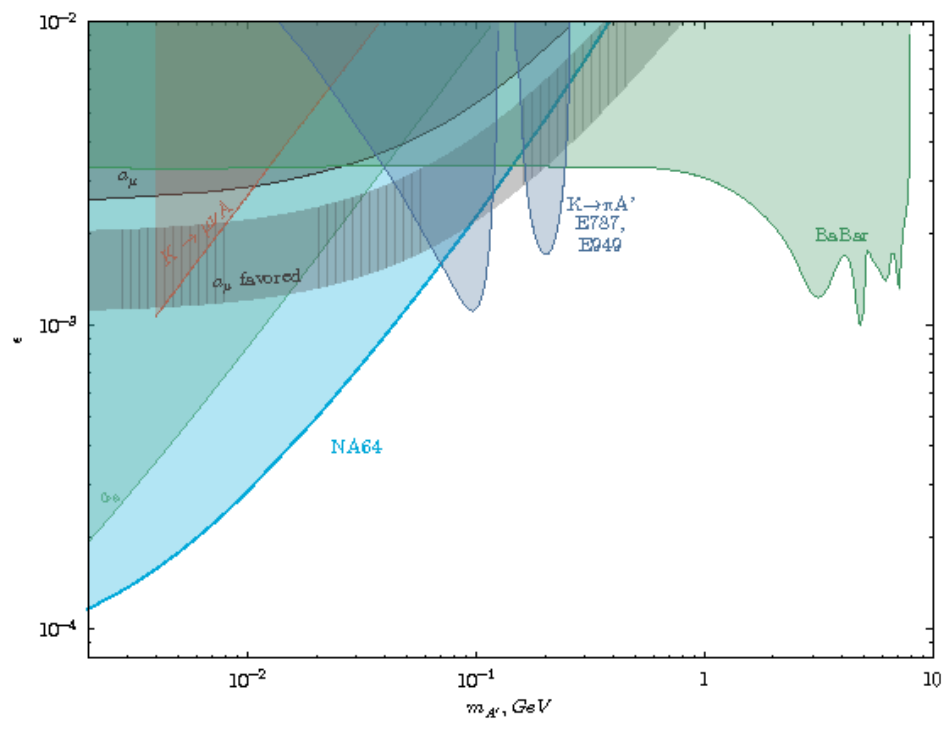

Figure 2.5.1.: Limits on the dark photon from invisible decay product searches via missing mass [90].

vs. coupling strength parameter space, as illustrated in Fig. 2.6.3.

\subsection{Conclusion}

There is a very active interest in experimental dark photon search since the 1980s. A lot of progress has been made to cover the hospitable coupling-mass parameter space. However, it has to be kept in mind that the exclusion limits derived from the data are based on irreducible assumptions, making those limits rather model dependent. 

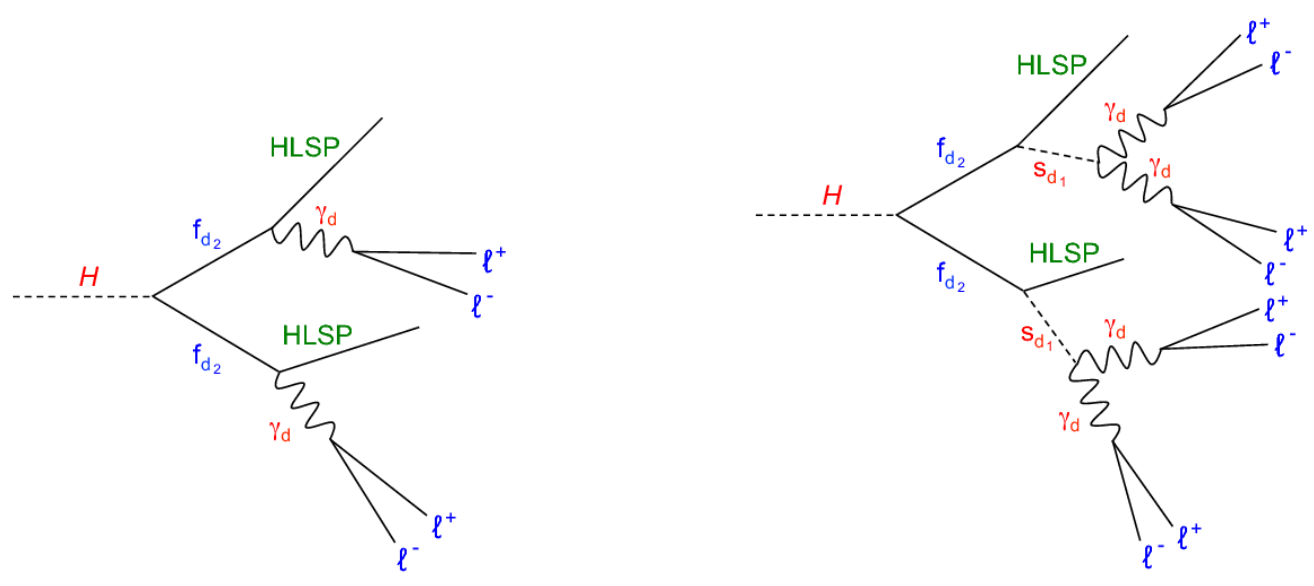

Figure 2.6.1.: Feynman diagrams of $\left(l^{+} l^{-}\right)$lepton jet production via a $\gamma_{D}$ dark photon [102].

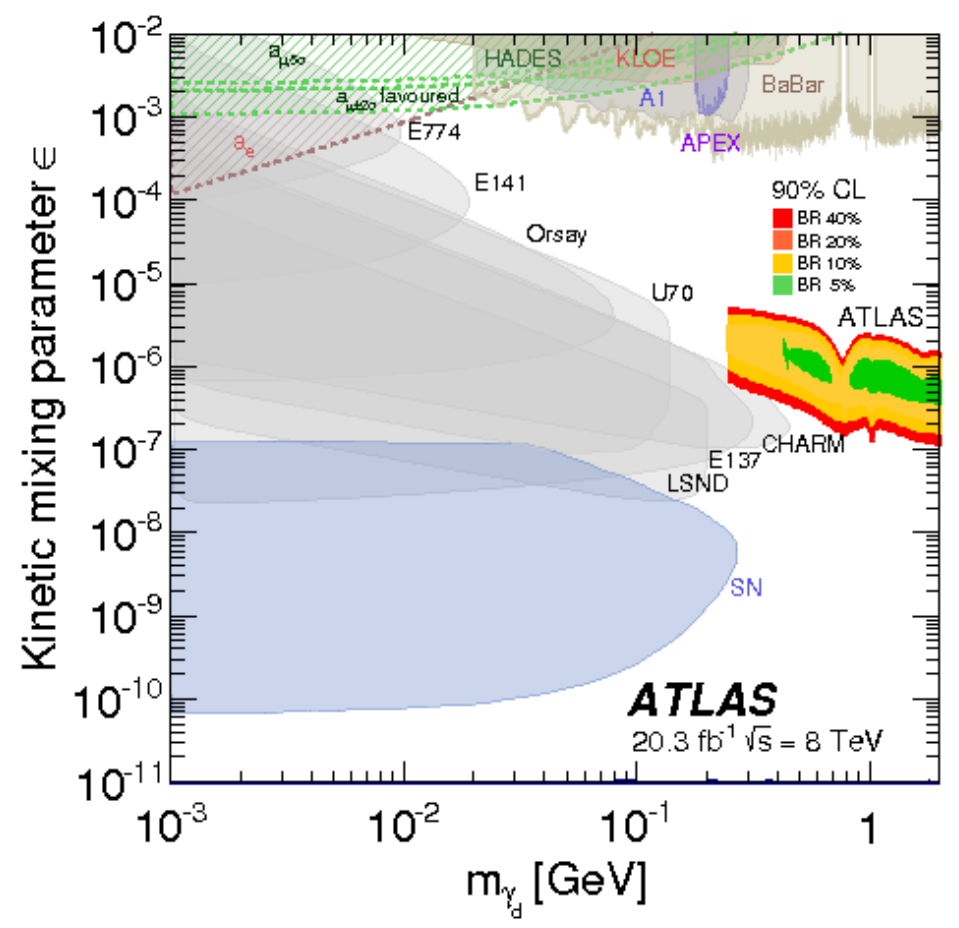

Figure 2.6.2.: Upper limits for a dark photon decaying into lepton jets kinetic mixing parameter vs. mass parameter space [102]. 


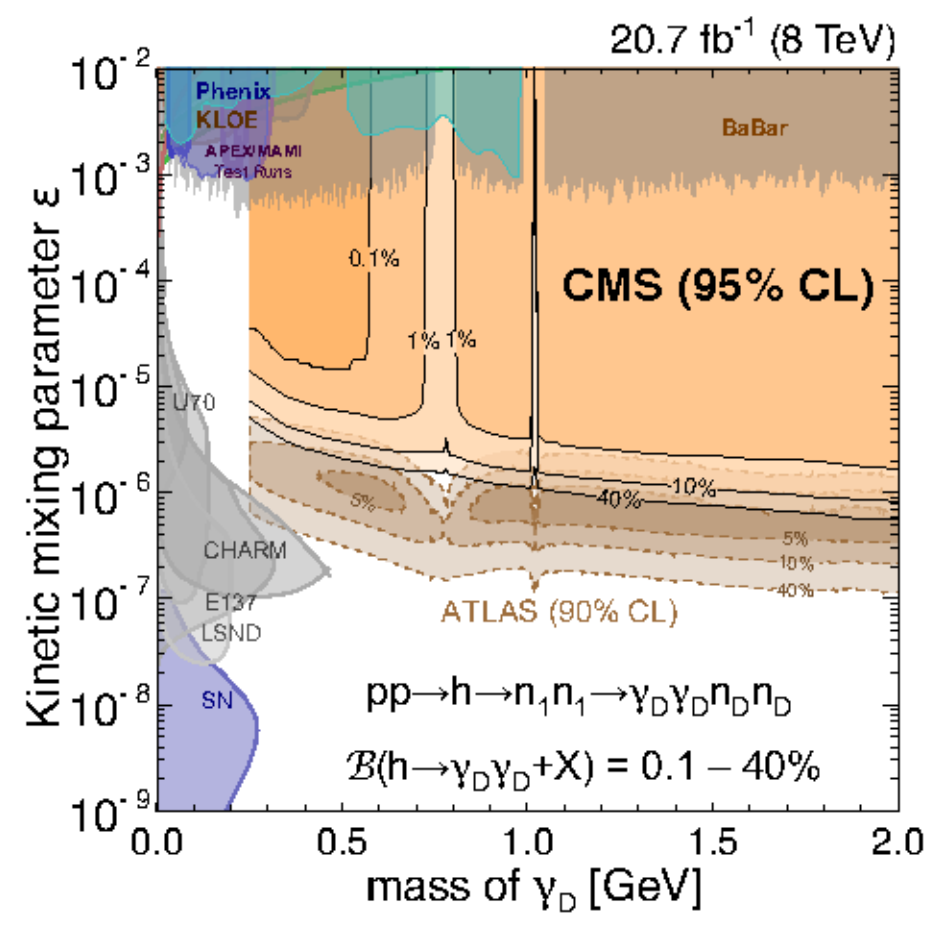

Figure 2.6.3.: Upper limits from CMS for a dark photon kinetic mixing parameter vs. mass parameter space [103]. 


\section{Detector Setup and Performance}

\subsection{Introduction}

The Thomas Jefferson National Accelerator Facility (JLab) is an electron accelerator center on the east coast of the USA (Newport News, Virginia). The continuous high-intensity electron beam is delivered to four experimental halls-A,B,C,D that are devoted to the investigation of various subjects in nuclear physics. HPS is an experiment conducted in the Hall B. The detector setup of HPS is based on the experience of the test run performed in May 2012. More details about the 2012 test run setup can be found in the HPS proposal documents [85]. We took some first data in 2014-2016. They are the focus of this thesis. The data taking will continue in the coming years. In this chapter, the experiment and the apparatus are described along with the trigger and the data acquisition systems.

\subsection{The CEBAF Accelerator}

The continuous electron beam accelerator facility (CEBAF) has been performing since 1994 with beam energies up to $6 \mathrm{GeV}$. After a decade of highly productive research activity and physics results, new questions motivated an upgrade to double the beam energy. The upgrade started in 2008 with the construction of a fourth experimental hall (Hall D). Major equipment upgrades were made in other halls as well to support a broad program of nuclear physics, like, for instance, hadron spectroscopy, the exploration of the quark-gluon dynamics and the multidimensional structure of the nucleon. The upgrade was officially completed in 2017. Regarding HPS, we had some first engineering runs for HPS in 2014, 2015 and 2016.

The CEBAF accelerator, shown in Fig. 3.2.1, consists of two linear accelerators connected by circulating arcs and an electron injector. The linear accelerators are based on the superconducting radio frequency (RF) technology. Each linac consists of 25 cryogenic modules supported by cooling helium liquifiers. Each cryomodule consists of eight RF cavities each of which contains five or seven cells, see Fig. 3.2.2. The acceleration capacity of each module provides an electron energy of $25 \mathrm{MeV}$ or $100 \mathrm{MeV}$, for the original and newly installed cryomodules respectively. The total acceleration is $1.1 \mathrm{GeV}$ per linac and hence $2.2 \mathrm{GeV}$ per full cycle. The number of revolutions depends on the needs of an experiment. 


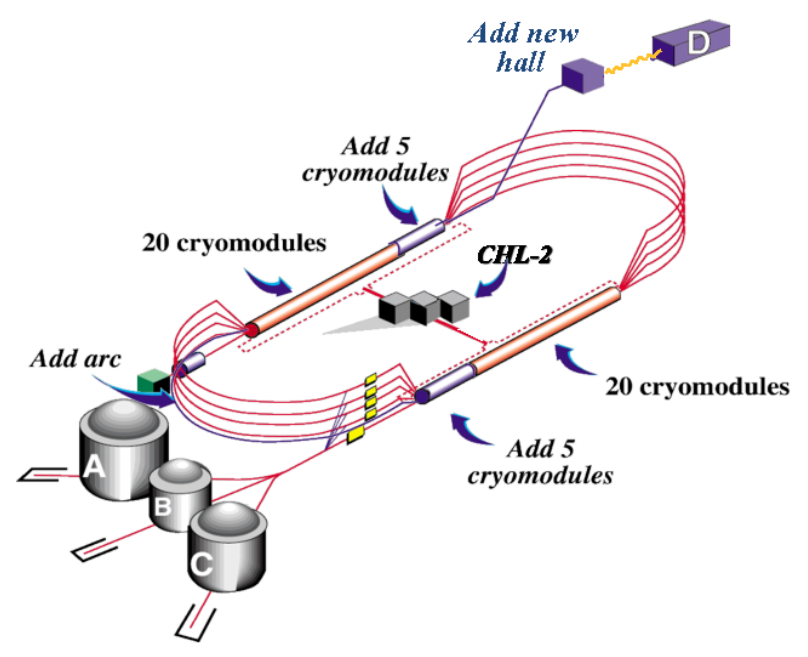

Figure 3.2.1.: The CEBAF accelerator with the A,B,C,D experimental halls.

The primary electron beam can be separated and delivered to the four experimental halls simultaneously with different energies and different intensities as high as $150 \mu \mathrm{A}$ [104, 105]. RF separators operating at $499 \mathrm{MHz}$ for halls A,B,C and $750 \mathrm{MHz}$ for hall $\mathrm{D}$ are used to deliver electron bunches.

The electrons in CEBAF are generated in the gun by photo-emission from a strained GaAs super lattice photo cathode. Four independent lasers produce pulses at a $500 \mathrm{MHz}$ frequency with controllable phase and amplitude, triggering the photocathode to shape independent pulse structures with their own controlled phase and current. Each experimental hall has a dedicated laser. The beams are combined before entering the accelerator and separated just before entering a hall. The laser system in CEBAF can switch between subharmonics of a $500 \mathrm{MHz}$ pulse, i.e. $250 \mathrm{MHz}$, $125 \mathrm{MHz}, 62.5 \mathrm{MHz}$, and $31.25 \mathrm{MHz}$ [106].

The ability of the accelerator to provide a nearly continuous high-intensity clean electron beam makes it an excellent tool for studying potential new physics with weak coupling strength such as heavy photon production. The continuity of the CEBAF beam in combination with high-resolution detector setup suppresses pileup effects. Electron bunches are delivered every $2 \mathrm{~ns}$, which is comparable to the time resolution of the trigger system. This means that the beam backgrounds are spread in time as uniformly as possible.

In the spring of 2015, HPS was planned to run with a beam energy of $\sim 2.2 \mathrm{GeV}$. The energy of an electron coming out of the injector is $100 \mathrm{MeV}$ and one pass through the double-linac system adds $2.2 \mathrm{GeV}$ to the beam energy. Therefore, during normal operation, the available beam energies at Hall $\mathrm{B}$ are $100 \mathrm{MeV}+2.2 \mathrm{GeV} \cdot \mathrm{n}$ where $\mathrm{n} \in[1,5]$. Due to a mechanical problem with one of the helium liquifiers of the cooling system right before the engineering run in the spring of 2015 the 


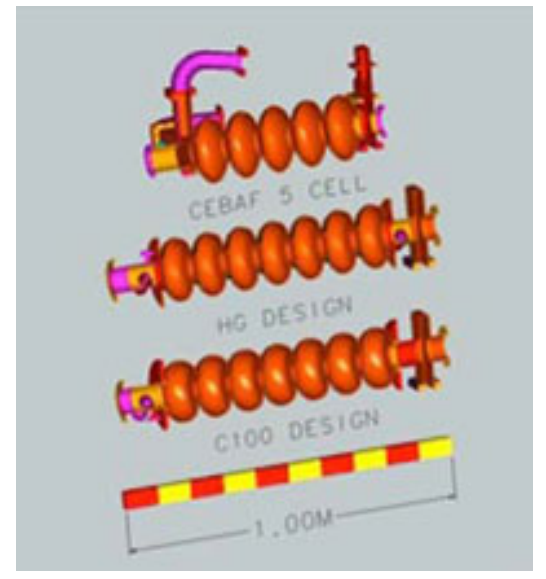

Figure 3.2.2.: A sketch of the different RF cavities designed for CEBAF. Combinations of five-cell (top) and seven-cell (bottom) low-loss RF cavities are used for the upgrade. The RF cavity depicted in the middle was found to heat up more than the low-loss RF cavities and was therefore not used.

superconducting cavities could run only at half of their nominal gradient. As a result, the accelerator could not provide beams with a $2.2 \mathrm{GeV}$ single-cycle energy, and therefore the HPS ran at a normally-not-available $1.056 \mathrm{GeV}$, shifting the original schedule. In the spring of 2016, the experiment ran finally with $2.2 \mathrm{GeV}$.

\subsection{The HPS Detector}

The HPS experiment requires a high precision measurement of two quantities: the invariant mass of the $e^{+} e^{-}$pairs and the position of their vertex. The physics of dark photon decay in $\gamma^{\prime}$ dark-strahlung on a thin target produces a highly boosted electron-positron pair with an opening angle related to the $\gamma^{\prime}$ mass:

$$
\theta_{e^{+} e^{-}} \sim \frac{m_{A^{\prime}}}{E_{0}}
$$

This process is accompanied by irreducible bremsstrahlung background processes with no preferred direction. Typical low-mass $\gamma^{\prime}$ s favor onward decays requiring greater sensitivity in small angles for the detector setup. Also on-shell $\gamma^{\prime}$ production followed by its di-electron disintegration leaves a displaced vertex as a signature. Therefore, for a dark photon search through this signature, an accurate vertex position reconstruction is essential.

The HPS setup is shown in Fig. 3.3.1. The target and the silicon vertex tracker (SVT) are set up in a vacuum chamber, installed within the spectrometer dipole magnet. The magnetic dipole field of the analyzing magnet points in the $Y$ direction, which is perpendicular to the $X Z$ beam plane. The whole system is mounted so that it provides flexibility to allow operations at different beam energies. The target 


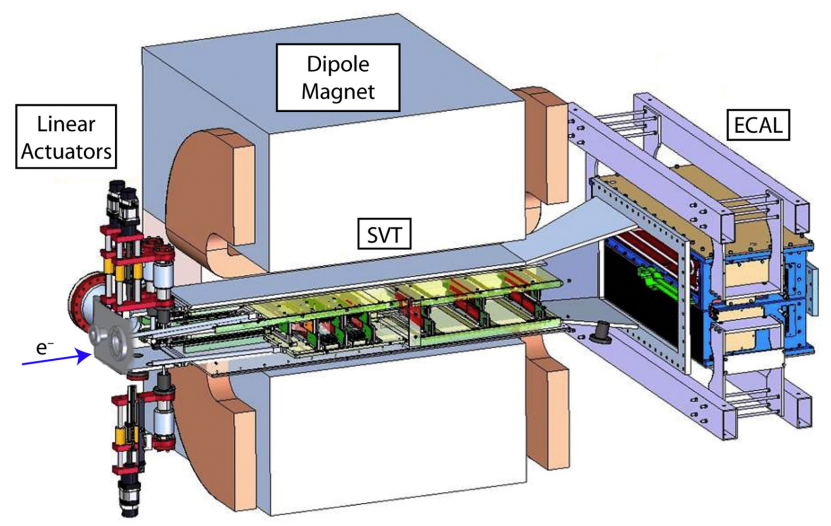

Figure 3.3.1.: Analyzing magnet and HPS detector setup.

foil is placed in the beginning of the high field of the analyzing magnet, followed by the layers of silicon detectors of the tracker and downstream of the magnet is the electromagnetic calorimeter (ECal), used for triggering and electron-positron identification. The ECal is placed at a distance $134 \mathrm{~cm}$ away from the target and the first row of each module is as close to the beam plane as $20 \mathrm{~mm}$. Since interaction of the primary beam with air or other medium produces a lot of low momentum secondaries, thus increasing the background and creating high occupancies, the SVT along with the target are placed in a vacuum chamber. The ECal vacuum chamber is connected to the SVT vacuum chamber and is designed to keep the beam remnants in vacuum. The detector setup is on the pathway of the primary beam, as the scattered beam particles and radiative secondaries are bent by the magnetic field into the horizontal plane - into a so-called "wall of flame". To avoid damage in the "wall of flame", both the tracker and the calorimeter are divided into 2 halves mirroring each other relative to the beam plane. The proximity to the primary beam also elevated the risk of a high pile-up rate, therefore, controlling the pile-up was one of the challenges of the experiment. This later concern also hinders tracking by pattern recognition due to high detector occupancies.

\subsubsection{The Beamline}

The beamline is split into two sections, the $2 \mathrm{C}$ line and the $2 \mathrm{H}$ line. The $2 \mathrm{C}$ line originates in the beam switch yard and follows the line bringing the beam from the accelerator to the hall, whereas the $2 \mathrm{H}$ line begins at the upstream end of the hall and leads to the beam dump. The end of the $2 \mathrm{H}$ beamline is illustrated in Fig. 3.3.2.

The HPS apparatus is set up downstream of the CLAS spectrometer. It is based on a three-magnet chicane system, as shown in Fig. 3.3.2, consisting of an analyzing magnet with a maximum magnetic field strength of $1.5 \mathrm{~T}$ and a pole length of $91.44 \mathrm{~cm}$, and two small dipole magnets that guide the beam back to the beam dump with a pole length of $50 \mathrm{~cm}$ and a maximum field strength of $1.2 \mathrm{~T}$. 
Beam Position Monitors (BPM) are installed at the beginning of the $2 \mathrm{H}$ line, followed by a collimator. The BPMs along with a harp scanner $2 \mathrm{HO} 2 \mathrm{~A}$, illustrated in Fig. 3.3.3, are used to monitor the beam position and beam profiles just before it enters the active experimental zone. The collimator protects the SVT layers in case of a beam excursion from a direct beam exposition. Quadrupole and dipole correctors are used to tune the beam. A pair of BPMs help to control the trajectory of the beam and test the inclination stability. Halo monitors set up before and after the spectrometer magnet are used to monitor the beam halo profile.

The chicane magnet system is separated by shielding walls from the final part of the beamline. Bremsstrahlung photons generated in the target continue through the detector system towards the photon beam dump, which is a tungsten inserted lead caveat $\sim 7 \mathrm{~m}$ from the target. The electron beam continues its journey further to the Faraday cup and the hall B electron beam dump behind a second shielding wall.

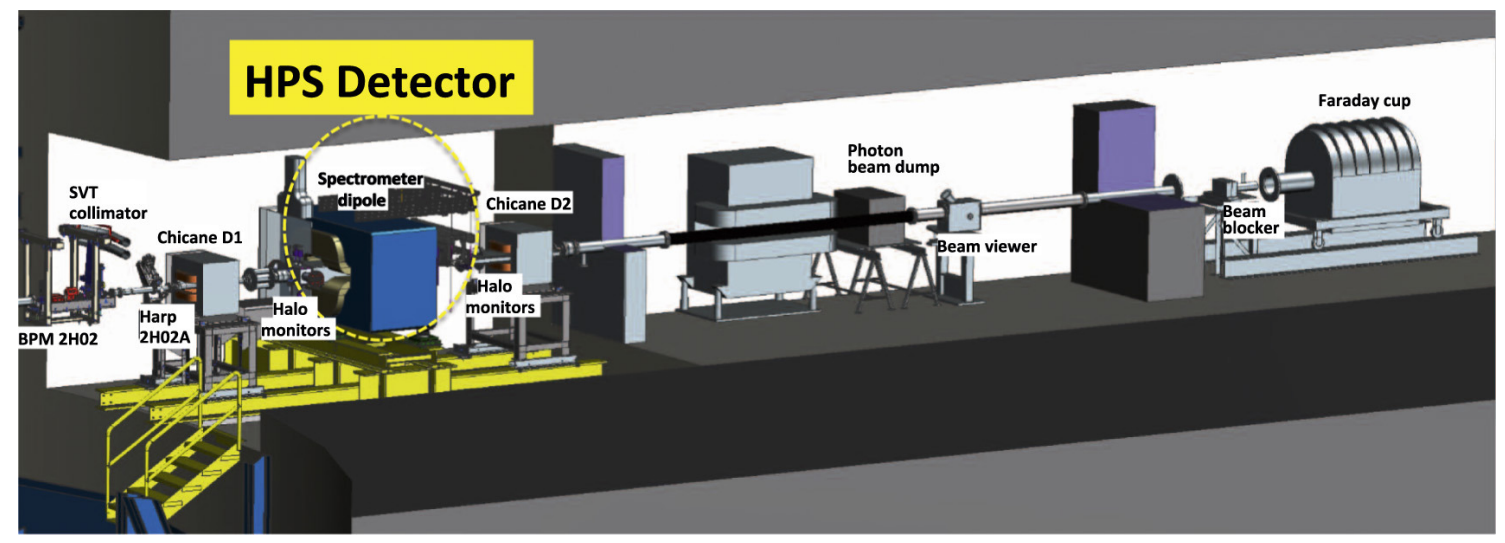

Figure 3.3.2.: HPS setup in the downstream area of the CLAS spectrometer [107].

\subsubsection{The Beam}

The beam profile and size are monitored with three wire scanners, two of which are installed in the tunnel. The first two (2C23, and $2 \mathrm{C} 24$ or tagger scanner) are used to tune the required beam parameters for the initial setup. The third scanner $(2 \mathrm{H} 00)$ is positioned in front of the first chicane magnet. The scanners are labeled after the names of the magnets they are next to. The tagger scanner is about eight meters away from the HPS target and has three round wires at $90^{\circ}, 45^{\circ}$ degree angles relative to each other. Beam profiles in $X$ and $Y$ direction are obtained from the wires at a $90^{\circ}$ angle. The third wire information provides an additional measurement that is used to calculate the major axis orientation of the beam profile, assuming an elliptical shape.

The Hall B tagger magnet is used to direct the beam away from the HPS apparatus during the beam tuning process. Once an acceptable beam profile is achieved, the 


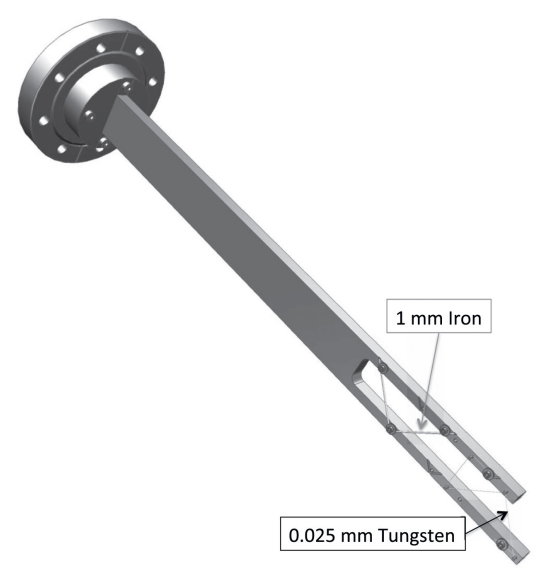

Figure 3.3.3.: Harp scanner used to monitor the beam orientation and profiles.

tagger magnet is turned off and the beam profile once again is checked by $2 \mathrm{H} 00$ scanner with the three wire configuration.

The physics of the dark photon production process discussed above requires a small beam size, since it allows one to measure the decay angles with better precision and to improve the vertex definition by adding geometrical constraints on the trajectory of a potential $\gamma^{\prime}$. Minimizing the beam cross-section also helps to minimize interactions of beam tails with the first layers of the tracker.

To study rare processes like dark photon production, HPS requires high luminosity, especially to be sensitive to small masses of $\gamma^{\prime}$. Therefore, a beam current of up to $500 \mathrm{nA}$ is used for HPS. However, this raises implementation issues. One of the most challenging is the deformation of the thin tungsten target at high temperatures stemming from the high intensity point-like beam. This was resolved by smearing the beam radius in the horizontal direction and minimizing it (the beam size) in the vertical one, thus maximizing the beam surface on the target (more details in the following subsection).

\subsubsection{The Target}

The choice of the target material was guided by the consideration of minimizing the hadronic production relative to QED radiative and $\gamma^{\prime}$ production. The ratio of QED to hadronic processes scales as $\left(\frac{Z^{2}}{A}\right)$ [63], and this motivates the use of a material with high $Z$. For HPS, a thin tungsten target is used with a $0.125 \%$ (or $\sim 4 \mu \mathrm{m}$ ) thickness placed $10 \mathrm{~cm}$ from the first layer of the SVT [108]. As shown in Fig. 3.3.4, the primary target is a square with $10 \mathrm{~mm}$ sides, supported by a thicker frame in the shape of an inverted " $U$ " to hold the target foils as flat as possible. It is loaded from above. The supporting frame constructed around the target was designed to be thin to reduce the radiation damage risk for the silicon strips in case of a beam excursion and to reduce the scattering of beam halo particles. 
The entire target system is constructed in four sections using two different thicknesses of tungsten foil, graphite and polyethylene [108]. The materials were chosen to be electrically insulant in order to avoid accidental eddy current effects. To be safe from the charge accumulated on the target foils from the secondary emission, a graphite wrapped ceramic plate with a thin wire is used to ground it. A second tungsten foil with a thickness of $0.25 \%$ radiation lengths is installed above the primary target and is used for beam current adjustments. A stepping motor linear actuator (similar to ones used for SVT silicon strips) makes the target movable during operation.

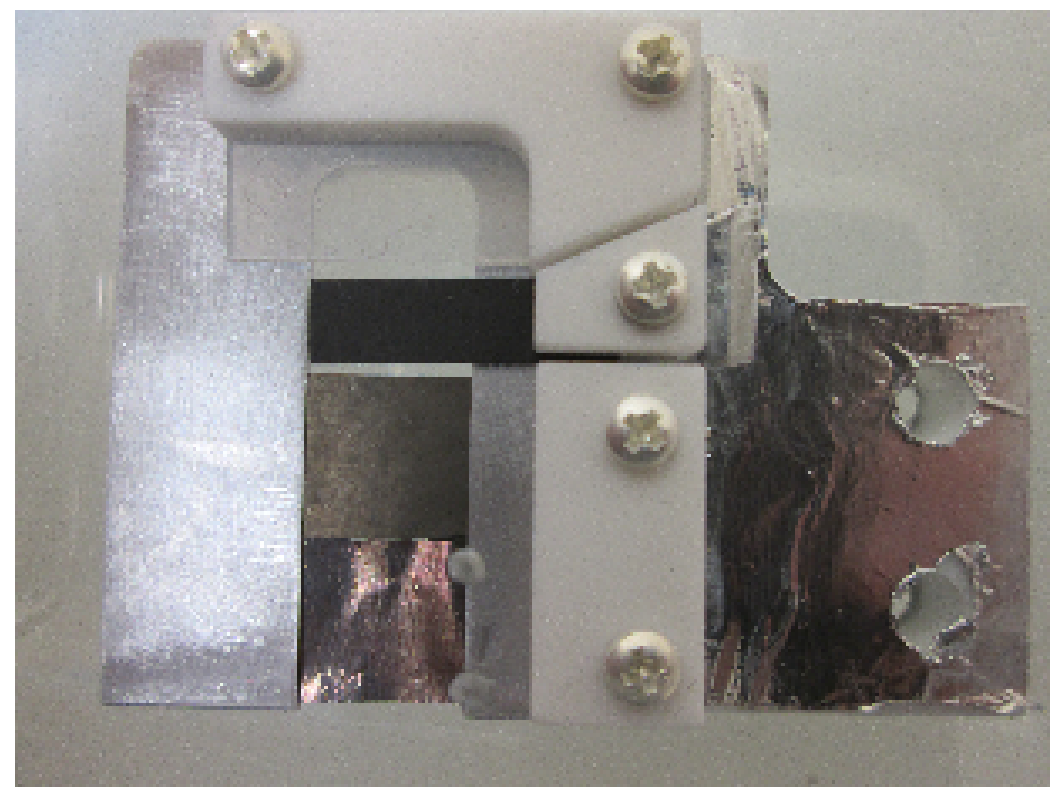

Figure 3.3.4.: HPS target and supporting system.

One of the issues arising from the use of a thin target and a high luminosity is the local heating. Each material in the HPS target assembly is vulnerable to this issue, especially polyethylene, which was used during the run in the spring of 2015 and was found to be damaged. The strength of tungsten reduces significantly with temperatures around $1000^{\circ} \mathrm{C}$. Because of the re-crystallization of the material, the probability of it developing cracks increases. The solution to this issue was to make the beam spot as large as possible. Together with the small beam size requirement discussed in Fig. 3.3.2, HPS ended up with a very asymmetrical elliptic shaped beam. This maximizes the beam spot on the target and avoids overheating. The small vertical dimension keeps the promise of a small beam size, thus providing better vertex resolution in the $Y$ direction, which is essential for forward-decaying dark photon detection. Studies with simulated data concluded that the beam with such a shape and dimensions does not affect the electron-positron pair reconstruction resolution for the purpose of a dark photon search. 


\subsection{The Tracker: Silicon Vertex Tracker}

The tracker consists of six double-layer of silicon strip sensors installed at 10 to $90 \mathrm{~cm}$ from the target, inside a vacuum chamber, see Fig. 3.4.1. The detector is split into two halves: top and bottom relative to the beam propagation line, so that each layer has top and bottom modules. The sensitive material in each layer consists of two silicon microstrip sensors placed with a small angle between them to provide two dimensional position measurements in the layer. The first three layers (1-3) are made of single sensor on each side with read-out only at one end. The remaining layers (4-6) are longer, to cover the curved trajectory of the particles that have drifted away from the center. The silicon strips of the 4-6 tracking layers are composed of two sensors, one on each side, and the read-out is done from both ends. In total, the SVT has 36 microstrip sensors.

Both groups of three layers in the top and bottom are attached on independent supporting plates. The plates for the layers 4-6 are stationary, while for layers 1-3 they can be opened and closed vertically. The support plates are installed into a support box that is embedded into the vacuum chamber itself.

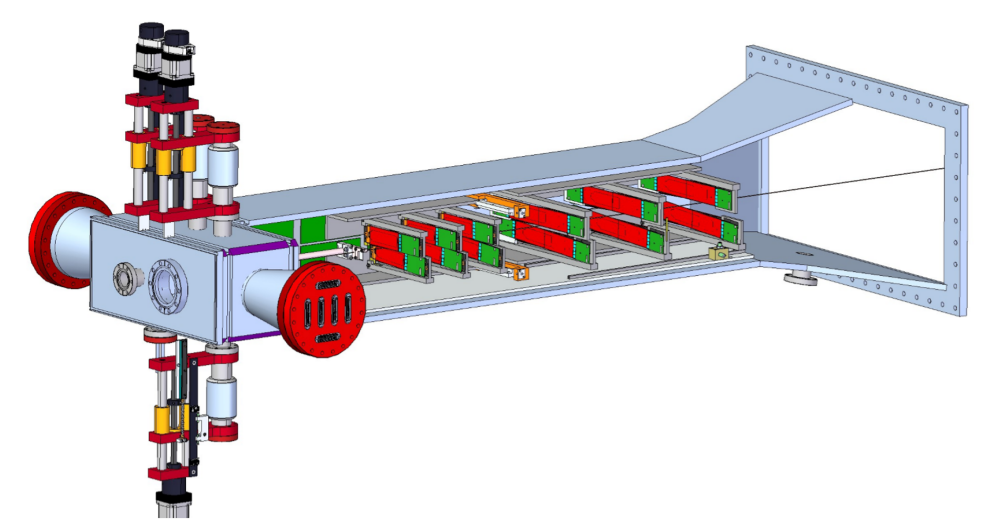

Figure 3.4.1.: Schematic view of silicon vertex tracker with supporting structures, top and bottom modules.

Sensitivity to low-mass $\gamma^{\prime}$ requires the minimization of the dead zone, to measure small angles. Monte Carlo simulations determined the closest achievable proximity. The physical edge of the SVT layer can be as close as $0.5 \mathrm{~mm}$ from the beam, while the sensitive material is at $1.5 \mathrm{~mm}$, once accounted for the carbon material. For the data-set considered in this analysis, the physical edge of the SVT was at $1.5 \mathrm{~mm}$. The reason for this was safety, as such detector proximity to the beam was never performed at CEBAF. Eventually, the distance of $0.5 \mathrm{~mm}$ was achieved, but is beyond the scope of this thesis. To minimize the effect of radiation damages, the silicon strips are actively cooled. A pair of chillers are used, one for the hybrids (circuit boards used in SVT readout system) and sensors at $-20^{\circ} \mathrm{C}$ and another one 
for the read-out boards at room temperature [109]. These have just a single cooling loop, while the hybrids and sensors have loops for both bottom and top halves.

In order to control the SVT position relative to the beam, a wire scanner, consisting of two thin-wires, is installed in front of the SVT. One horizontal wire $(20 \mu \mathrm{m})$ and another one at $9^{\circ}$ degrees w.r.t. the horizontal $(30 \mu \mathrm{m})$ are attached to the SVT frame before the first SVT layer, as shown in Fig. 3.4.2, so that they don't block the active region. These wires are used to precisely locate the beam position relative to the first layer of the SVT. This is used to get a high accuracy position measurement of the SVT relative to the beam.

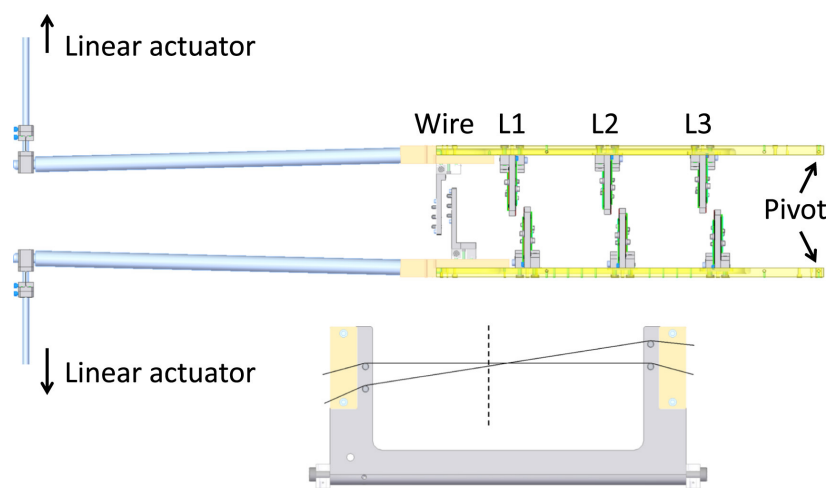

Figure 3.4.2.: Silicon Vertex Tracker with the wire system. The vertical dashed line represents the nominal position of the beam.[107]

More details about the design and operation of the SVT can be found in the SVT operation manual [109] and the HPS proposals [63, 86].

\subsection{The Electromagnetic Calorimeter}

The electromagnetic calorimeter of the HPS is a homogeneous calorimeter consisting of 442 lead-tungsten $\mathrm{PbWO}_{4}$ scintillating crystals that was built at the IPN Orsay. It is located right after the analyzing magnet.

\subsubsection{ECal Design}

The calorimeter design is illustrated in figure Fig. 3.5.1. This design was studied using the GEANT4 simulation toolkit [110]. The most efficient geometry was found to be a configuration with 10 rows of 46 lead-tungsten crystals. As simulations with the calorimeter simply attached to the SVT vacuum chamber exit window showed problematic background rates, the ECal is placed around a separate vacuum enclosure, made from aluminum. The region around the exiting beam path, where the most intense beam remnants propagate, is isolated from the calorimeter by similar aluminum plates. This helps to decrease the background as well. 
Like the tracker, the ECal is divided in two modules surrounding the beam plane. Each module is a mirror reflection of the other, thus consisting of 5 layers of 46 lead tungsten crystals, except for the first layer around the beam, which is missing nine crystals slightly off-center forming a hole in the beam propagation path. This is done to avoid the excessive electromagnetic background from primary scattered beam particles and radiative secondaries. The gap between the two halves of the calorimeter was measured to be $44 \mathrm{~mm}$ at the time of installation with a $0.3 \mathrm{~mm}$ error (vertical). After moving the calorimeter for fixes and other necessary operations on the detectors and supporting apparatus, a later survey showed slight deviations from this nominal value. The correction transformations applied in the simulation and reconstruction code, to account for them are described in sec.4.2.1

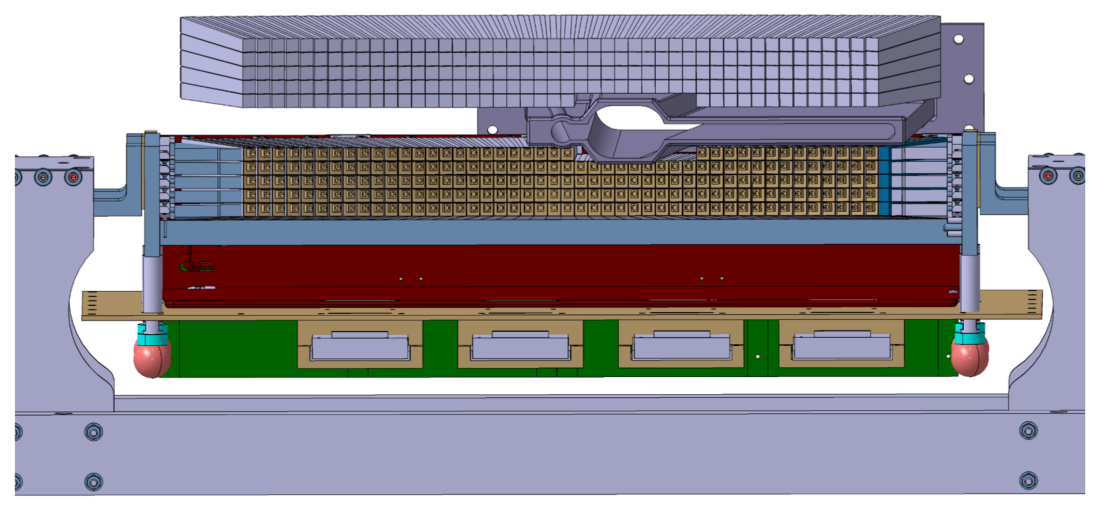

Figure 3.5.1.: Schematic view of the electromagnetic Calorimeter with the supporting structure and the top and bottom modules.

Operating in a high-background environment, the calorimeter has to provide a fast reliable signal to reduce pile-up effects. At the same time, it needs a light yield that satisfies the needs in term of resolution of the HPS. The short decay time of lead tungsten crystals meet these demands, even though the light yield is modest for them. The ECal is designed for detecting electrons and positrons in the $0.3-6.6 \mathrm{GeV}$ kinematic range, providing an energy resolution of the order of $\frac{4 \%}{\sqrt{E}}[111$.

\subsubsection{Signal Processing in the ECal}

The light yield from each crystal is read out with avalanche photodiodes (APDs) paired with custom preamplifiers. The schematic figure of a single crystal with its read-out system is shown in Fig. 3.5.2. A single crystal is a cuboid with uneven base squares of the following dimensions: $160 \mathrm{~mm}$ long, which is 18 radiation lengths, a front face of $13.3 \times 13.3 \mathrm{~mm}^{2}$, a rear face of $16 \times 16 \mathrm{~mm}^{2}$. Each is wrapped in a VM2002 reflecting foil to enhance the light collection at the back. To the rear face of the crystal a, one Hamamatsu $10 \times 10 \mathrm{~mm}^{2}$ avalanche photodiode is attached. It is plugged to the preamplifier, supported by a connection board that is also acting 
as a thermal screen. The signal is sent to the data acquisition (DAQ) system using a mother board.

Bi-colored light-emitting diodes (LED) are installed on the front face of each crystal. These are used for testing and monitoring the performance of the ECal both on and off line.

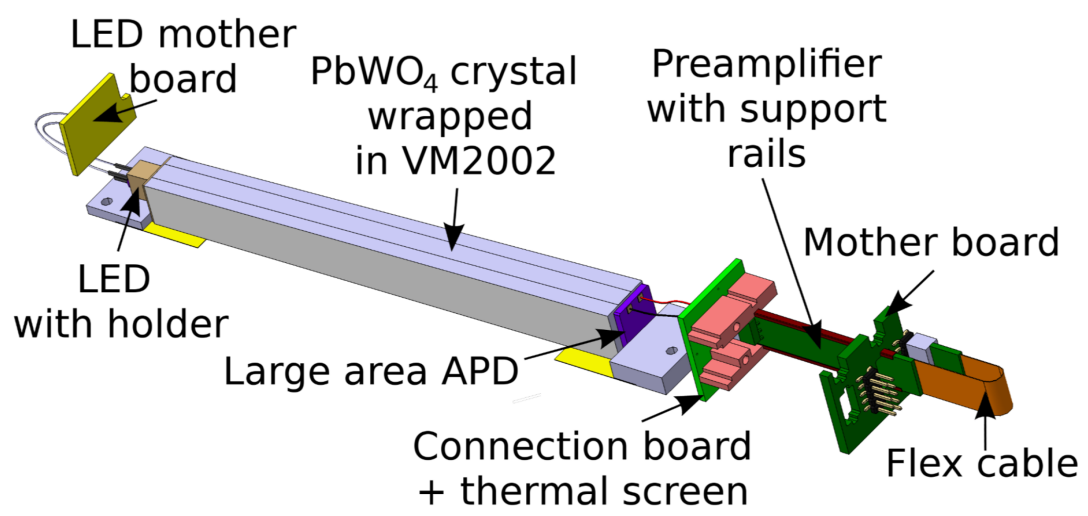

Figure 3.5.2.: Single crystal with it's read-out chain.

Since both the crystals and APDs are temperature-sensitive, to stabilize the gains, each half of the calorimeter is enclosed in a box with a controlled temperature. For this, a chiller is used, that runs a circulating water system in these boxes, hence preserving a temperature of $17 \pm 0.3^{\circ} \mathrm{C}$ [111].

The gain and leakage currents of the APDs depend on the bias voltage and temperature linearly. The properties of each photodiode were measured, in order to find the operating voltage for gain of 150 . Then the APDs were sorted in groups of $4-10$ with similar gain/voltage ratios. This allows one to power each group by one high voltage channel. The bias voltage of each APD group is chosen so that the gain of an APD is on average 150 at a temperature of $18^{\circ} \mathrm{C}$, corresponding to APD bias voltages set to $385-405 \mathrm{~V}$. An APD is connected to a preamplifier where the current is converted to a voltage with low noise and impedance. The gain of the preamplifier was configured so that the maximum energy deposited in a crystal would not be altering the amplitude converter, which is capped at $2 \mathrm{~V}$ [11]].

In the "crystal - avalanche photodiodes - preamplifier" processing sequence, the typical signal values exchanged are [111]: 120 photons/MeV for the light yield in the crystal, an APD quantum efficiency of 0.7 , an APD gain of 150, and a preamplifier gain of $0.62 \mathrm{~V} / \mathrm{pC}$ (for a $10 \mathrm{~ns}$ input pulse width). The low noise level of a few millivolts provides the possibility to set the threshold on each crystal individually (as low as $7.5 \mathrm{MeV}$ ). The output from preamplifiers goes through the four mother boards. Each module of the calorimeter is split into 26 groups for high voltages, each group is composed of APDs with similar gains, so that after voltage adjustment, the groups end up with a total gain uniformity of only a few percent. 


\subsubsection{JLab FADC250 in ECal}

The next stage of the signal processing is digitization. For this, JLab Flash ADC ${ }^{1}$ (FADC) boards are used. The pulse from an APD passes through the FADC module with a $250 \mathrm{MHz}$ clock and timing samples are saved with 12 - bit resolution in an $8 \mu \mathrm{s}$ "deep" pipeline. This is a $250 \mathrm{MHz}$ flash ADC developed for the JLab $12 \mathrm{GeV}$ upgrade. The amplitude of the signal, the timing and spatial information from each crystal are provided for the trigger decision described below. By initial design of the experiment, $\mathrm{TDCs}^{2}$ were installed for time measurement as well. Therefore, two-thirds of the signal from the preamplifier was delivered to a discriminator with built-in scalers and to the TDC channel. The remaining one-third was sent to the FADC input ${ }^{3}$.

The ECal is providing the trigger signal for HPS. Performing in high a background environment, it is important that the ECal is fast and covers as much of the acceptance region as the SVT.

\subsection{Trigger and Data Acquisition}

The HPS trigger algorithm is implemented on field-programmable gate array (FPGA) based trigger processors, that were used for the first time for HPS. The timing and energy information from FADCs are passed to the Crate Trigger Processor (CTP) that searches for clusters within a time coincidence window in both top and bottom halves. The final decision, made in the CTP and Sub System Processor (SSP), is reported to the read-out system of the tracker and calorimeter to respond correspondingly. The high resolution timing gives the advantage of making the coincidence window much tighter and of lowering the background. This is important for decreasing the rates in the detector to steerable numbers.

The trigger signal is used by the data acquisition (DAQ) system to manage data collection from the tracker and calorimeter. For the SVT, the Advanced Telecom Communication Architecture (ATCA) is used and the ECal is read by hardware based on VXS. The primary signal to trigger is coming from the ECal, after which it sends the signal to the other detector to read the event. Fig. 3.6.1 describes the trigger system schematically in a block diagram.

After trigger signal, the ECal Readout Controller (ROC) receives the information data from the detectors already in a digitized mode and distributes it to the Event Builder, which is a single blade Intel-based CPU module operating on CentOS Linux OS. The ROC application for the ATCA system, that is used for the SVT read-out,

\footnotetext{
${ }^{1}$ Analog to Digital Converter

${ }^{2}$ Time to Digital Converter

${ }^{3}$ This is true for the 2015 run analyzed in this thesis. Later on, the TDCs were uninstalled and the full signal was sent to the FADC only.
} 


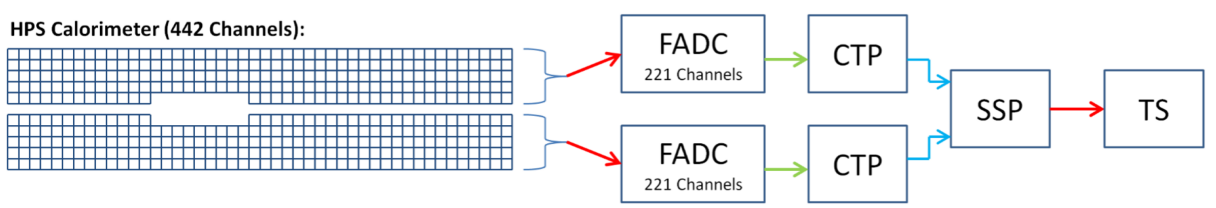

Figure 3.6.1.: The ECal trigger system.

is running on an integrated processor located on the ATCA mainboard. The Event Builder collects the information from the SVT and ECal, and constructs events and stores them in a RAID $5^{4}$ data storage system that can operate at a data rate of up to $100 \mathrm{MB} / \mathrm{s}$. The event Builder runs on Intel-based multi-CPU servers. A Foundry router supports high speed communication between the DAQ system elements and the JLab computing service. The SVT is the most "busy" detector, therefore the read-out controller is provided with a $10 \mathrm{Gbit} / \mathrm{s}$ link to the router. For the ECal, the connection allows 1 Gbit/s, which is more than enough for the HPS rates.

\subsubsection{The Trigger System}

The goal of the HPS trigger is to efficiently reject non- $e^{+} e^{-}$events or events not in the kinematic range of interest using the ECal information. For this, the trigger is searching for time coincidences of clusters in the top and bottom modules of the ECal. Moreover, specific kinematics are required for these clusters that are characteristic to $\gamma^{\prime}$ production.

The first step of the trigger logic uses the FPGAs on the FADC boards that report the crystal energy and time data to the CTP (Fig. 3.6.2). Using a 3 bit time information, the CTP can search in a 4 ns coincidence window. However, for HPS, an 8 ns window is set. The CTP and Sub-System Processor (SSP) are used to settle for the final decision. Then the Trigger Supervisor is incorporated to generate all the necessary signals and control the DAQ system using the Trigger Interface (TI) units that are available on every crate that is part of the read-out.

The trigger is designed to operate without a dead-time window at the rates expected for HPS, but it is flexible enough to apply dead time if necessary. The system can handle rates of more than $50 \mathrm{kHz}$ with a latency of approximately 3 microseconds, that is set to couple the requirement coming from the SVT APV25 chip.

The logic for triggering on a $e^{+} e^{-}$pair is summarized in the following three steps:

- For each channel, an FADC is sampling and converting the analog signals to digital ones, and reports the measured energy and timestamp of the pulse above a threshold to the CTP.

\footnotetext{
${ }^{4}$ Redundant Array of Independent Disks
} 


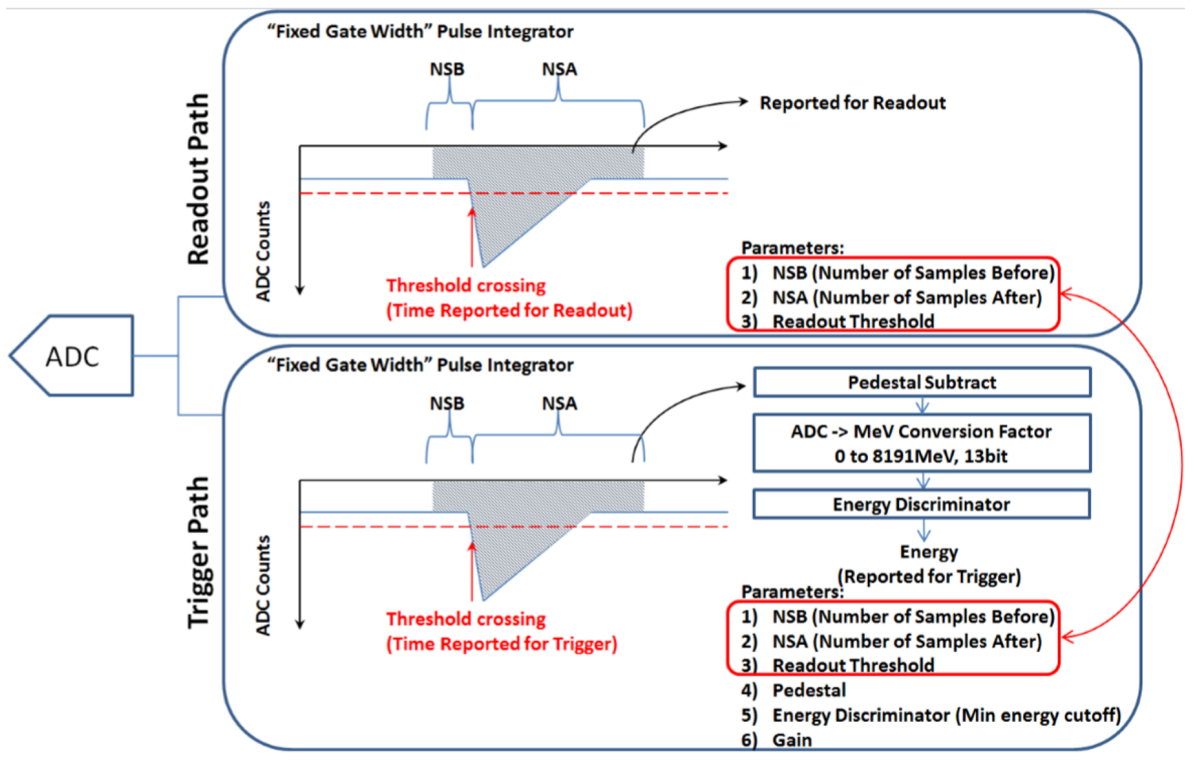

Figure 3.6.2.: ECal readout, FADC and trigger paths.

- A CTP performs cluster finding. For this, it groups the pulses received from an FADC, for the top and bottom ECal modules independently, into clusters and passes the energy, arrival time and hit pattern to the SSP.

- The SSP runs the pair searching algorithm, pairing up clusters from top and bottom, taking into account the time coincidence window and other kinematical cuts detailed below.

The cluster finding algorithm performed in the CTP is doing a simultaneous search along the crystal array for 125 clusters at once with a size of up to $3 \times 3$. The algorithm for cluster searching can be broken down to the following steps:

1. All the hit energies in $3 \times 3$ size channel windows are summed.

2. Hits are grouped together if their corresponding timestamp is within the set coincidence time window.

3. The grouped channels energy is required to be larger than the cluster energy threshold and more than the summed energy of any other neighboring $3 \times 3$ group of channels.

After these three requirements are met, the CTP sends the parameters of the cluster to the SSP. There is a CTP for both halves analyzing all hits every 4 ns. The flexible bigger coincidence window is set to consider hits that are excluded from this initial time window as a part of the cluster, since this time-off could come from electronics and signal distribution in the cables.

For the overlapping clusters and larger than $3 \times 3$ size clusters, additional information is sent to the SSP to filter: 
- Cluster energy - 13 bit (in $\mathrm{MeV}$ ),

- Cluster position in terms of crystal index $(\mathrm{x}, \mathrm{y})$,

- Cluster time (4 ns resolution),

- Cluster $3 \times 3$ hit pattern (the channels that reported a hit in the cluster)

The cluster position is defined as the position of the crystal carrying the most energy in the cluster. Information about the hit pattern is used by the SSP to analyze and reject the strange patterned groups of hits or perform a low resolution cluster centroid calculation [112].

The SSP receives the information from the two modules of the calorimeter and filters the clusters to select $e^{+} e^{-}$candidates corresponding to $\gamma^{\prime}$ decay kinematics. For this, the following selection criteria are applied [112]:

1. Energy sum of clusters is within $\left[E_{\min }, E_{\max }\right]$.

2. Clusters time coincidence is within $\left|t_{\text {top }}-t_{\text {bottom }}\right| \leq \Delta t_{\text {max }}$

3. Clusters have comparable energies $\left|E_{\text {top }}-E_{\text {bottom }}\right| \leq \Delta E_{\text {max }}$

4. Energy slope, $E_{\text {cluster }}-R_{\text {cluster }} \times F_{\text {energy }} \leq T_{\text {slope }}$

5. Co-planarity check $\left|\tan ^{-1}\left(\frac{X_{t o p}}{Y_{\text {top }}}\right)-\tan ^{-1}\left(\frac{X_{\text {bottom }}}{Y_{\text {bottom }}}\right)\right| \leq \theta_{\text {coplanarity }}$

6. Threshold for number of hits in $3 \times 3$ window, $N_{\text {hits } 3 \times 3} \geq T_{\text {hit }}$

where $E_{\text {cluster }}$ is the energy of the cluster with minimum energy, $R_{\text {cluster }}$ is the distance of that cluster from the detector center, $F_{\text {energy }}$ is a parameter, $T_{\text {slope }}$ and $T_{\text {hit }}$ are the threshold parameters of the energy slope and of the number of hits, $\theta_{\text {coplanarity }}$ parameter is checking the linear property of two clusters on XY plane. All these parameters can be programmed according to the run requirements. The SSP is configurable to a single cluster based trigger decision, which is used for commissioning and calibration runs.

\subsubsection{SVT Data Acquisition}

The SVT DAQ system is supporting the continuous $41.667 \mathrm{MHz}$ read-out and processing of signals from the 36 silicon strip sensors of the SVT [113]. Then, it passes the events along for further processing by the JLab DAQ. It is designed to handle large amounts of data due to the high expected occupancy in the detector. To reduce pile-up effects, we have a sampling of the pulse heights in six consecutive time buckets for every hit. This helps to reconstruct the hit timestamps with a good precision. The read-out system includes a front-end board in the vacuum for power distribution and digitization of the signal. Being in the vacuum causes cooling issues, but reduces interferences by reducing cable length.

Every sensor is linked to a hybrid board using five 128-channel APV25 front-end ASICs. In Fig. 3.6.4, one of such hybrids is shown. It was used during the 2012 test 
run. Every hybrid board is connected to the front-end read-out board through five analog lines carrying low power LVDS differential current pulses. The preamplifier at the front-end readout board multiply the differential current from the APV25 to match it within the range of a $14-$ bit ADC. One front-end board is operating for four hybrids. The digital signal output goes to the compact 8-pair mini-SAS cables and connects with vacuum flanges. Then, outside the vacuum chamber, they connect to the external DAQ. The front-end readout boards are connected to the FPGA to have access to the clock and trigger information.

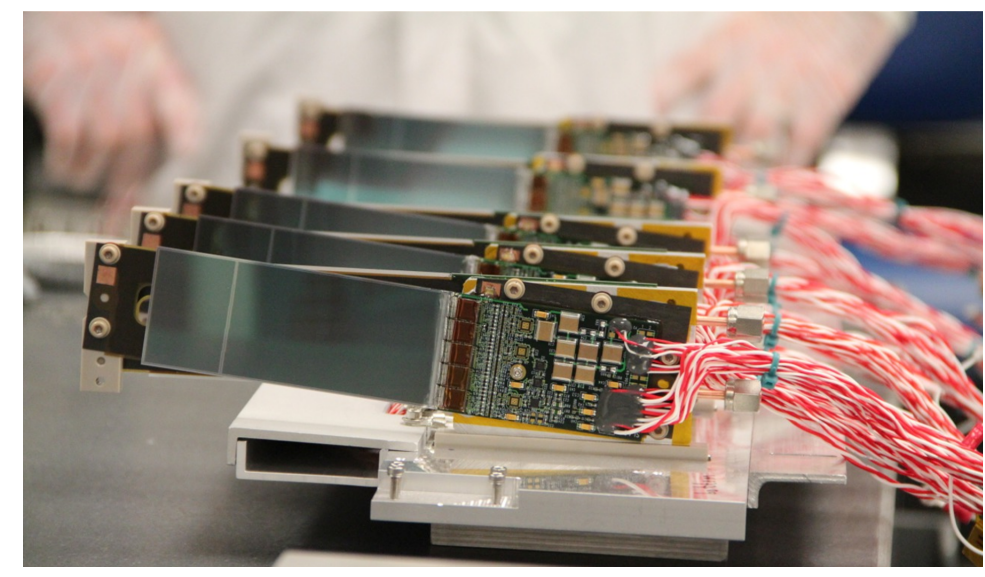

Figure 3.6.3.: SVT Hybrid board from the 2012 test run.

To transfer the signal through optical connections to the ATCA crates, the digitized signals are converted on custom constructed flange boards outside the vacuum flange. Each board has optical drivers to carry out electrical-optical conversion and send it through optic fibers to the ATCA crate. Fig. 3.6.4 summarizes in a schematic plot the SVT DAQ system. Finally, $10 \mathrm{Gbit} / \mathrm{s}$ Ethernet cables are used to send the data to the JLab DAQ. The maximum rates for the SVT is about $50 \mathrm{kHz}$, which is the operating limit of the APV25.

\subsubsection{ECal Readout with Flash ADC250}

Once the trigger signal is received, the data is accessed and if the signal passes the threshold cut, the selected number of samples before $(\mathrm{NSB}=5)$ and after $(\mathrm{NSA}=25)$ the trigger are transmitted [111]. The schematic diagram in Fig. 3.6.2 explains this process and the FADC two parallel data paths: the trigger path and the readout path. The trigger path is continuously processing the signals to report the hits to the trigger manager, whilst the read-out path sends the hits to the DAQ system only after a trigger was received.

The technical properties of the FADC modules are [112]: 


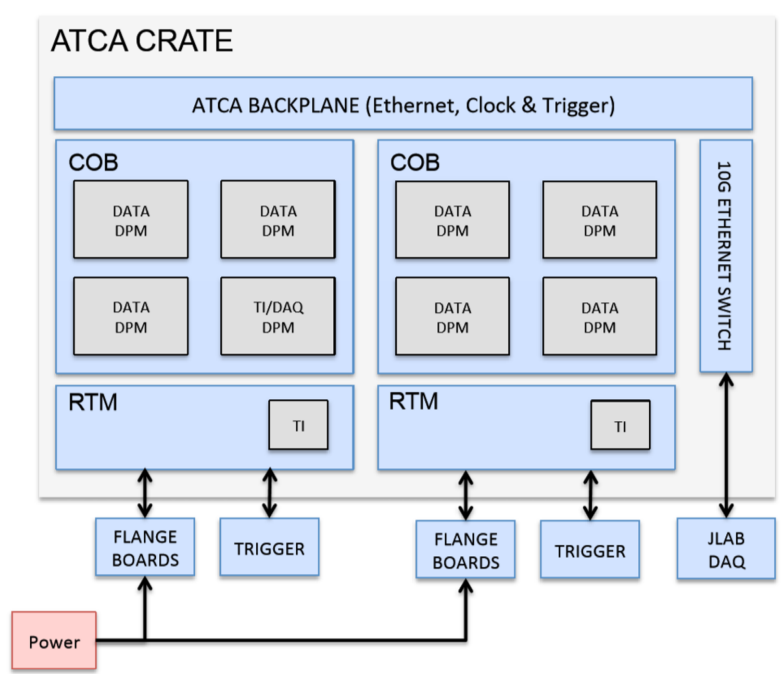

Figure 3.6.4.: SVT DAQ system.

- A sampling rate of ${ }^{5} 250 \mathrm{Msps}$

- A 12 - bit digitization

- $50 \Omega$ for the termination input

- $-0.5 \mathrm{~V},-1 \mathrm{~V},-2 \mathrm{~V}$ front end input $(-2 \mathrm{~V}$ is used in HPS)

- A nominal charge per ADC of 10 to $39 \mathrm{fC}$

The trigger path has not only NSB and NSA information for each channel, but also the trigger threshold (in ADC counts) and a pedestal. The conversion factor, often referred as "gain", is used for the trigger to convert the ADC counts to MeV energies. The trigger has an independent energy threshold, that is used to select the pulses before sending the signal to the CTP. A gain conversion factor and a pedestal value are provided to calculate the hit energy. This information is reported to the CTP every 32 ns. If the hit doesn't occur in these $32 \mathrm{~ns}$, a NULL energy pulse is sent. This 32 ns window defines the worst case pulse resolution equal to 32 ns for a single channel, but smaller if the pulses are in 32 ns consequent time windows .

\subsubsection{HPS Triggers}

Two pair triggers (Pair-0, Pair-1), two single triggers (Single-0, Single-1) and random pulser trigger are operating in parallel providing signal for read out. Each configuration has independent selection cuts.

Single triggers are used to study the detector performance and background rates. The Single-0 trigger is designed to report one-cluster events in

\footnotetext{
${ }^{5}$ Mega-Samples Per Second
} 
wide interval of energies. The single- 1 trigger is responding to elastically scattered particles, carrying nearly full beam energy.

Pair triggers are creating pairs from a two-clusters registered in opposite modules of the ECal. Pair-0 is used to select Moller scattered electrons, while Pair-1 is used as the main trigger to the HPS signal. Pair-1 triggers on electron-positron pairs, based on the set of kinematic cuts implemented for 2 clusters detected in different ECal modules.

The selection criteria for each configuration of the triggers are detailed in [111]. The leakage effects are not corrected at this level and need to be accounted for in the setting of the trigger configuration.

\subsection{Conclusion}

The HPS detector is equipped with a silicon vertex tracker and an ECal for dark photon search. The electromagnetic calorimeter provides fast trigger signal with time resolution, allowing to lower the rate of background processes due to the continuous beam operation. The tracker properties allow for close detection of the particles after decay, maximizing the acceptance and providing secondary channel for dark photon search via its displaced vertex. The tracker information is used for particle identification and for the energy measurement of $e^{+} e^{-}$pair. 


\section{Detector Simulation and Reconstruction}

\subsection{Introduction}

Simulation, calibration, and reconstruction are essential parts of a physics experiment. The simulation of physics channels within a detector system helps to draw conclusions about the experimental reach, establish the detector setup with the largest efficiency, and anticipate any possible implementation issues. The calibration allows for the signals measured in the detector to be re-adjusted relative to each other or relative to a base signal, in order to equalize the responses of all detector channels or have an absolute normalization. The reconstruction is based on the detector simulation and calibration. It derives physics information based on the signals measured in all electronic channels. Implementing a precise geometrical model of the detector setup is key for this step. In addition, calibration-based corrections, and various other improvements, are performed in order to make sure that the detector gives a representation of the underlying physics as accurately as possible.

Dark photons in HPS are detected through their dielectron decay products. The key parameters, the invariant mass distribution of the $e^{+} e^{-}$pair and the location of the decay vertex, are reconstructed from the hits of the electron and positron registered in the layers of the SVT and in the crystals of the ECal for tracking and energy measurements, respectively. The processing of the analog signal from each detector was explained in the previous chapter. During the reconstruction, the ECal clustering algorithm takes the data to form the clusters of energy deposits from the hits recorded in each ECal module. In parallel, the tracking algorithm is processing hits from the SVT layers for track finding. Afterwards, a track-cluster matching algorithm groups the clusters into tracks induced by the same particle. Vertexing uses each electron-positron pair kinematics to reconstruct different interaction vertices, one constrained to the target, one constrained to the beam spot, and one unconstrained.

During the reconstruction, the information provided by the detector simulation, the detector calibration, and the kinetic corrections for the clusters and tracks, are all taken into account. Orsay group has built and supports the ECal, so my thesis was mainly focused on the ECal performance studies. Therefore, this chapter is 
offering a short overview of the ECal detector simulation followed by a discussion on reconstruction, corrections, calibration, and finally, resolution studies.

\subsection{The Ecal Geometry Simulation}

Particles propagating through detectors are simulated with a software called SLIC ("Simulator for the Linear Collider" [114]), which is based on the GEANT4 MC toolkit [110] and python. The detector geometry description in SLIC is input via an XML text file and parsed at runtime through the LCCD ("Linear Collider Detector Description") software, which is an extension of GDML ("Geometry Description Markup Language" [115]) developed by the LHC Applications Group. SLIC receives standard stdhep ${ }^{1}$ input from the event generator (MadGraph4 [116], MadGraph5 [117]) and outputs LCIO [118] format files. These files are run through the HPS software to output data with reconstructed physics information. HPS is employing custom-written software in java where the detector description for simulation and for reconstruction are implemented in two different files. The data can later be converted to a ROOT format or processed within the LCIO application framework for $\mathrm{C}++$ or Java.

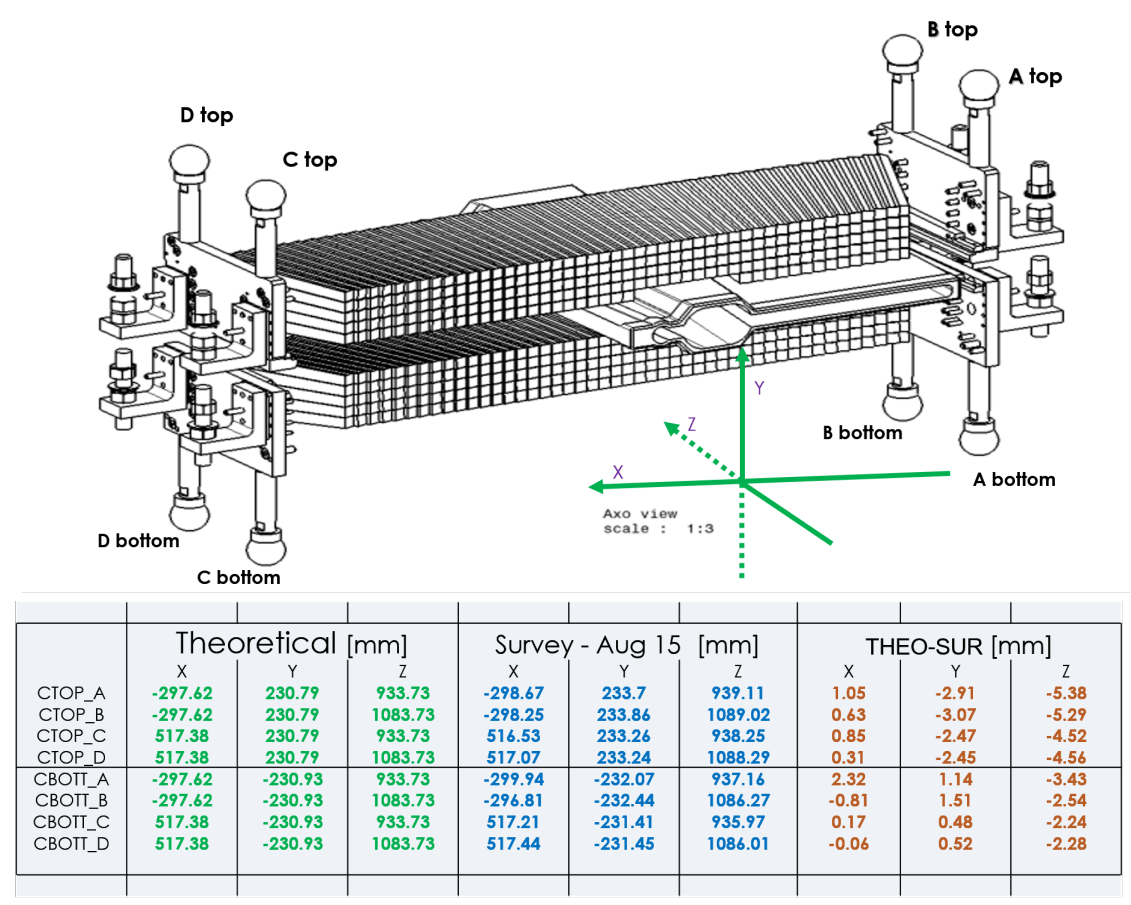

Figure 4.2.1.: In the top a schematic drawing of the ECal with the eight targets used for position measurements located in the corners (see main text). In the bottom, the measurement results are tabulated.

\footnotetext{
${ }^{1}$ Binary file for Monte Carlo event records (i.e. HEPEVT) in high energy physics.
} 
To understand the backgrounds and detector efficiencies, the simulation and reconstruction chains described above are very important. It was found that the reconstructed cluster energy from data and from simulation vary depending on the precise distances of the clusters from the edges of the calorimeter. To correct for this effect, it is important to know the deviation between the real position of the ECal and its theoretical position, to adapt the simulation. Surveys to find the exact position of the calorimeter were, therefore, performed. The detector exact position was measured using four fixed survey-points on each half of the ECal module, A, B, $\mathrm{C}$, D, shown in Fig. 4.2.1. The nominal coordinates and the survey results from the last measurement on August 15, 2015 are shown in the table also in Fig. 4.2.1. The difference between the theoretical (nominal) and the survey position are presented in the last three columns of the same table.

Measurements during the ECal assembly also showed a small asymmetric displacement of the crystals from their nominal position within the ECal volume (left plot in Fig. 4.2.2). The weight of the crystals introduced another effect making the ECal modules sag about $\mathrm{dY}=40 \mu \mathrm{m}$ in the front frame with respect to the target and $\mathrm{d} Y=90 \mu \mathrm{m}$ in the back frame, as illustrated in the right plot of Fig. 4.2.2. All these distortions from the nominal configuration could contribute to differences in simulated and reconstructed real data. The parametrization of the necessary geometrical corrections and an overview of their implementation in the detector simulation code are described in the following.

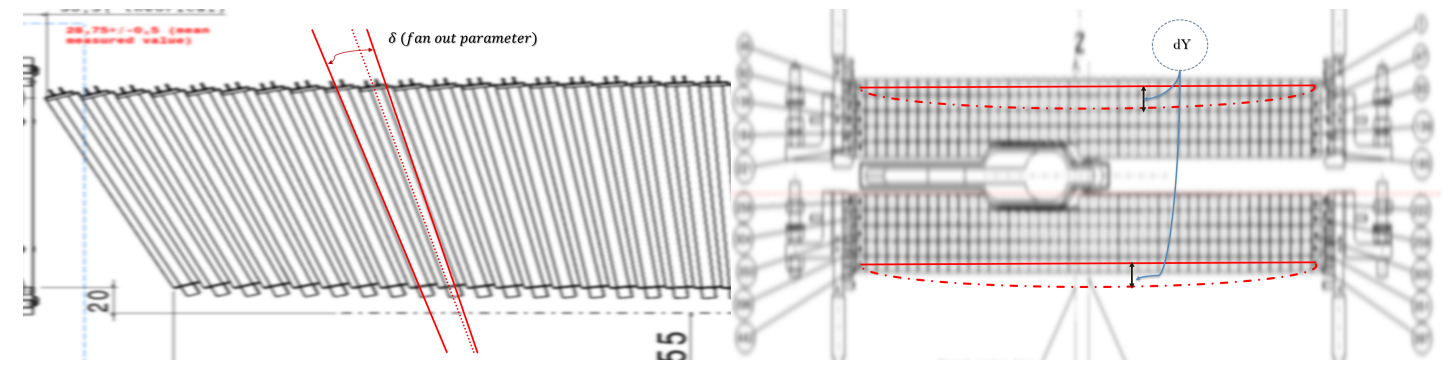

Figure 4.2.2.: Illustration of the asymmetrical shift of the calorimeter crystals and the sagging.

\subsubsection{ECal Geometry Correction}

The survey results study concluded that the A, B, C, D (Fig. 4.2.1) benchmark points are not coplanar. Therefore, as a first step, all possible planes formed by each triplet of points in the set A, B, C, D are considered. From the parameters of these planes, an averaged plane is derived. The relative inclination angles between these planes are calculated (Fig. 4.2.3), including a comparison to the nominal position plane (horizontal). All angles are small, below $0.2^{\circ}$, indicating that no significant tilt is present, allowing one to approximate the corrections for the average plane with sufficient accuracy. 


\begin{tabular}{|c|c|c|c|c|c|c|}
\hline angle[deg] & ABC plane & ABD plane & ACD plane & BCD plane & $\begin{array}{c}\text { ABCD } \\
\text { average } \\
\text { plane }\end{array}$ & $\begin{array}{c}\text { Nominal } \\
\text { plane }\end{array}$ \\
\hline ABC plane & & $\mathbf{0 . 0 1 2 7}$ & $\mathbf{0 . 0 6 8 8}$ & $\mathbf{0 . 0 6 9 9}$ & 0.03496 & $\mathbf{0 . 0 6 8 5}$ \\
ABD plane & 0.0236 & & $\mathbf{0 . 0 6 9 9}$ & $\mathbf{0 . 0 6 8 8}$ & 0.03498 & $\mathbf{0 . 0 7 5 1}$ \\
ACD plane & 0.1278 & 0.1304 & & $\mathbf{0 . 0 1 2 6}$ & 0.03495 & $\mathbf{0 . 0 3 1 8}$ \\
$\begin{array}{c}\text { BCD plane } \\
\text { ABCD }\end{array}$ & 0.1626 & 0.1771 & 0.0902 & & 0.03493 & $\mathbf{0 . 0 4 4 2}$ \\
$\begin{array}{c}\text { average plane } \\
\text { Nominal plane }\end{array}$ & 0.0680 & $\mathbf{0 . 0 7 7 6}$ & $\mathbf{0 . 0 6 4 1}$ & $\mathbf{0 . 0 7 3 7}$ & & $\mathbf{0 . 0 7 3 7}$ \\
\hline
\end{tabular}

Figure 4.2.3.: Relative angle between the planes formed by the A, B, C, D benchmark points for top and bottom ECal modules, including the average plane derived from all possible triple-point planes.

The geometric corrections applied to the simulation are calculated between the nominal and the average planes and presented through parallel translation vector and rotation vector. In total, this correction uses six parameters for each half of the calorimeter: three for translations and another three for rotations, presented in Tab. 4.2.1. Applying this correction to the known nominal positions of the crystals (based on the design from the IPN Orsay engineer Emmanuel Rindel) we obtain the final geometrical placement of the crystals. This map is generated relative to the calorimeter, whilst the placement in the detector simulation is relative to the target. So the map of the crystal positions is shifted with respect to the target position in the main coordinate system - $(21.5,0.0,1361.45)[\mathrm{mm}]$. The placement

\begin{tabular}{lccc}
\hline Field Name & Translation Vector [mm] & Field Name & Rotation Matrix [rad] \\
\hline \hline "top_tr_x" & -0.71 & "top_rot_alpha" & 0.0006496 \\
\hline "top_tr_y" & 2.7245 & "top_rot_beta" & 0.0 \\
\hline "top_tr_z" & 4.9375 & "top_rot_gamma" & -0.0004688 \\
\hline \hline Field Name & Translation Vector [mm] & Field Name & Rotation Matrix [rad] \\
\hline \hline "bot_tr_x" & -0.405 & "bot_rot_alpha" & 0.000515 \\
\hline "bot_tr_y" & 0.9125 & "bot_rot_beta" & 0.0 \\
\hline "bot_tr_z" & 2.6225 & "bot_rot_gamma" & 0.001347 \\
\hline
\end{tabular}

Table 4.2.1.: Translation vectors and rotation matrices for ECal top (top table) and bottom (bottom table) modules' transformations with respect to the nominal position of the crystals. The field names used in the geometry description files are denoted as $\alpha$-rotation around the $X$ axis, $\beta$-around the $Y$ axis and $\gamma$-around the $Z$ axis. 
of the geometrical volume in GEANT4 is given by the coordinates of the center and by the rotation matrix in Tait-Bryan angles $\overrightarrow{\mathrm{k}}(\phi, \theta, \psi)$ defining the direction of the detector volume axis. Taking into account this information, the rotation matrix is applied to the unit vector defining a crystal, which is along the axis passing through its front and back square-sections.
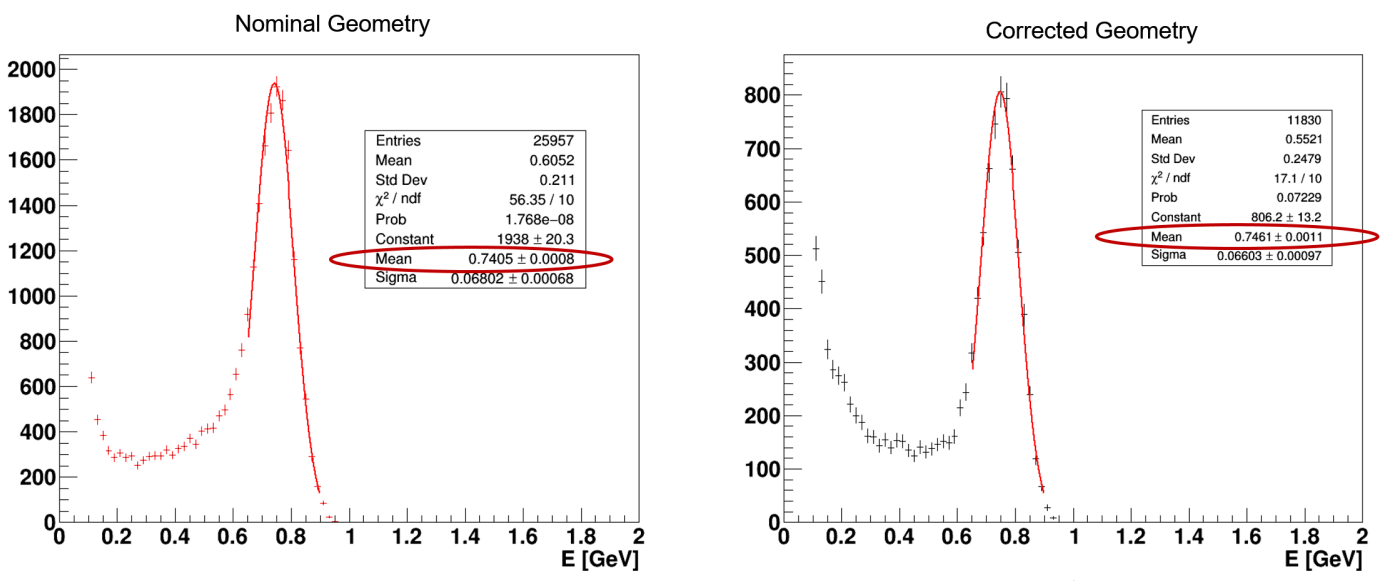

Figure 4.2.4.: The reconstructed cluster energy fit with a Gaussian function (red line) using the nominal geometry (left) and the corrected geometry configuration (right).

By initial design of the geometry code, the ECal detector was configured in a codependent rigid scheme, challenging independent transformations of separate modules. Thus, the main effort was directed towards making the ECal detector configuration flexible in order to introduce the geometrical corrections in a user friendly, easily modifiable input source. This is also important for future runs, to be able to configure the detector position in the geometry software accordingly. Additional fields were added to the detector data constants in the compact.xml file in the detector-data folder in the Hps-Java package. The input fields of the file can be accessed based on the name-titles in Tab. 4.2.1.

The cluster energy distribution using the updated geometry description is plotted and fit with a simple Gaussian Fig. 4.2.4. The differences between the Gaussian mean values gives an estimation of the geometrical correction effect, which is $\approx 1 \%$ of the cluster energy.

\subsection{Event Reconstruction}

The reconstruction algorithm analysis uses information from the SVT and the ECal to find tracks and clusters respectively. Using this information, the track-cluster matching algorithm finds the track-cluster pairs most likely created by the same 
charged particle. Then the vertexing algorithm is run to couple each pair of particles to a single vertex. Based on the kinematics, the software makes collections of $e^{+} e^{-}$ pairs from QED tridents and $e^{-} e^{-}$pairs from Moller scattering. The latter are used to calibrate the detectors and study the mass resolution.

\subsubsection{SVT Hit Reconstruction}

A particle detected in the SVT deposits a charge in the strips of the sensor. Samples per strip from each signal form a temporal distribution of the deposited charge in a sensor. Six such samples are considered as a SVT hit signal and are fitted using a 3-pole function

$$
f(\mathrm{t})=\mathrm{A} \frac{\tau_{1}^{2}}{\left(\tau_{1}-\tau_{2}\right)^{3}}\left(e^{-\frac{t-t_{0}}{\tau_{1}}}-\sum_{k=0}^{2}\left(\frac{\tau_{1}-\tau_{2}}{\tau_{1} \tau_{2}}\right)^{\mathrm{k}} \times \frac{e^{-\frac{t-t_{0}}{\tau^{2}}}}{\mathrm{k} !}\right),
$$

where $\tau_{1}$ and $\tau_{2}$ describe the rise and fall of the signal, $A$ parametrizes the amplitude, $t_{0}$ represents the hit time and $t$ is the sample timing

A nearest neighbor algorithm is used to cluster the hits with the following logic:

1. All raw hits are considered and hits with an amplitude significantly above the noise threshold are selected as cluster seed hits: $A_{\text {hit }}>4 \times \sigma_{\text {noise }}$.

2. Neighboring strips within a $8 \mathrm{~ns}$ time window with respect to the seed hit timing and amplitude $A_{\text {hit }}>3 \times \sigma_{\text {noise }}$ are found and added to the seed hit. This search is repeated until no more such strips are found. The resulting collection of the hits are considered as a cluster candidate.

3. Step 2 is repeated until no more seed strips are found.

4. From the found candidates, a cluster is rejected if the summed signal of hits in a cluster is not significant.

5. Clusters in the neighboring layers are grouped within a 16 ns timing window. Using the stereo angle between the layers gives a 3D hit that is used in the track finding algorithm.

\subsubsection{Track Reconstruction}

Several strategies are used for track reconstruction depending on the chosen seed layers: the minimum number of hits requested for a track, kinematic constraints, and the maximum $\chi^{2}$ accepted. The following steps outline the typical track reconstruction algorithm:

1. All combinations of 3D hits from 3 seed layers are considered to form initial 3 -hit tracks. These tracks are fitted and filtered according to a $\chi^{2}$ requirement. 
2. The remaining tracks are "extended" by adding hits from the rest of the layers one by one. The hits are accepted if the new track satisfies a $\chi^{2}$ requirement.

3. Finally, the tracks are filtered based on their timing: $t_{R M S}<8 \mathrm{~ns}$. A Generalized Broken Lines (GBL) algorithm fit is eventually performed to model multiple scatterings and provide better precision.

Several strategies (i.e. combinations of seed layers) of seed layers are used to maximize the track efficiency and ensure that all good tracks are found.

\subsubsection{ECal Hit Reconstruction}

The energy deposited in the crystal and its timestamp are derived from the pulse information. Depending on the approach, the read-out configuration of the FADC can be in the following modes:

- Mode 1 - The full waveform information is recorded with all hits and the pulse analysis is performed offline,

- Mode 3 - Fixed NSB and NSA number of samples before and after passing the threshold are used to calculate the deposited energy,

- Mode 7 - The FADC performs the pulse analysis and provides timing, pulse integral, pedestal and pulse maximum information.

The threshold for hits is the same for all modes and set to 12 FADC counts. Mode 3 and Mode 7 have been considered to reduce the data stream in case of high rate; in particular, the trigger uses the information from mode 3. However, only the mode 1 was used in the 2015 engineering run, described in this thesis, since the data quantity, even with full waveform, was not too large for the DAQ.

The time stamp of a hit reconstructed in mode 3 has a 4 ns intrinsic resolution. In this mode, the timing has the largest deviation per crystal with an important time walk effect. Time-walk is the delay observed for small signals as they take more time to reach threshold. The read-out window size in mode 1 is $200 \mathrm{~ns}$ (50 samples) with the trigger pulse center at about $\sim 50 \mathrm{~ns}$. For all 3 read-out modes the pedestals are not deducted by the FADC. The trigger system uses map of pedestals generated with specific runs to correct for them.

To extract hit information in mode 1 , the full waveform is parametrized using a 3-pole function of the following form [119] :

$$
f(t)=P+\frac{A}{2 \tau^{3}}\left(t-t_{0}\right)^{2} e^{-\frac{\left(t-t_{0}\right)}{\tau}}
$$

where $P$ is the pedestal, $A$ is the amplitude of the pulse with $A=0$ if $t<t_{0}$, $t_{0}$ is the time of the hit, and $\tau$ is representing the timing of the pulse maximum. The pedestal is calculated using the samples before the threshold. The energy and 


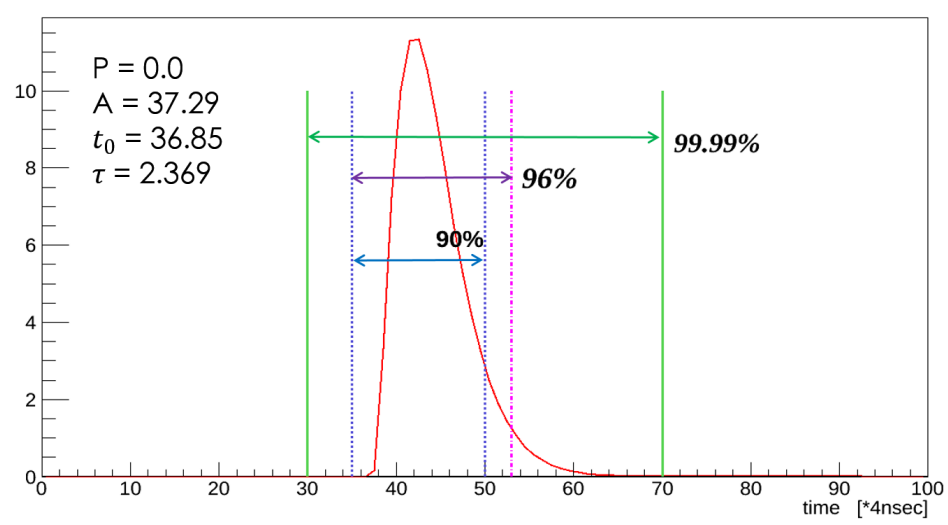

Figure 4.3.1.: $f(t)$ function with parameters estimated from the fit to the hits in the ECal to find the signal window containing $99 \%$ of the pulse. The lines represent the suggested cuts for the window size around the maximum value.

time of a hit are obtained from the fit to the full waveform distribution from the parameters $A$ and $t_{0}$ respectively.

For the mode 3, it was important to define the window of $\mathrm{N}$ samples, containing full pulse information. Using the eq.(4.3.2) form to model the pulse shape, we found the typical parameters from the cosmic ray signals, in order to define an optimal window for the FADC samples. In Fig. 4.3.1, we show various possible cut choices and the quantity of signal collected in each case.

\section{Study of Pile-up in ECal}

An estimation of pile-up effects in the ECal were studied with data collected in December 2014 using the $A^{\prime}$ pair trigger setting with a beam current of $50 \mathrm{nA}$, $100 \mathrm{nA}, 150 \mathrm{nA}$, and $200 \mathrm{nA}$. The number of signals with an additional (pile-up) peak were divided by the number of signals with just a single peak for each crystal. The results show a small contamination due to pile-up across the calorimeter, mostly below a few percent, even at higher luminosity (Fig. 4.3.2). One of the factors contributing to a low pile-up level is the shape of the function used to model the signal in the crystal which is highly efficient in rejecting the additional pile-up peak.

\subsubsection{Track-Cluster Matching Algorithm}

Identifying the matching tracks and clusters is the next problem to solve in order to fully reconstruct a particle. The tracks reconstructed in the SVT are extrapolated to the ECal and the coordinates at the calorimeter are compared with the cluster coordinates. The spacial difference of track versus cluster is used as a discriminating 

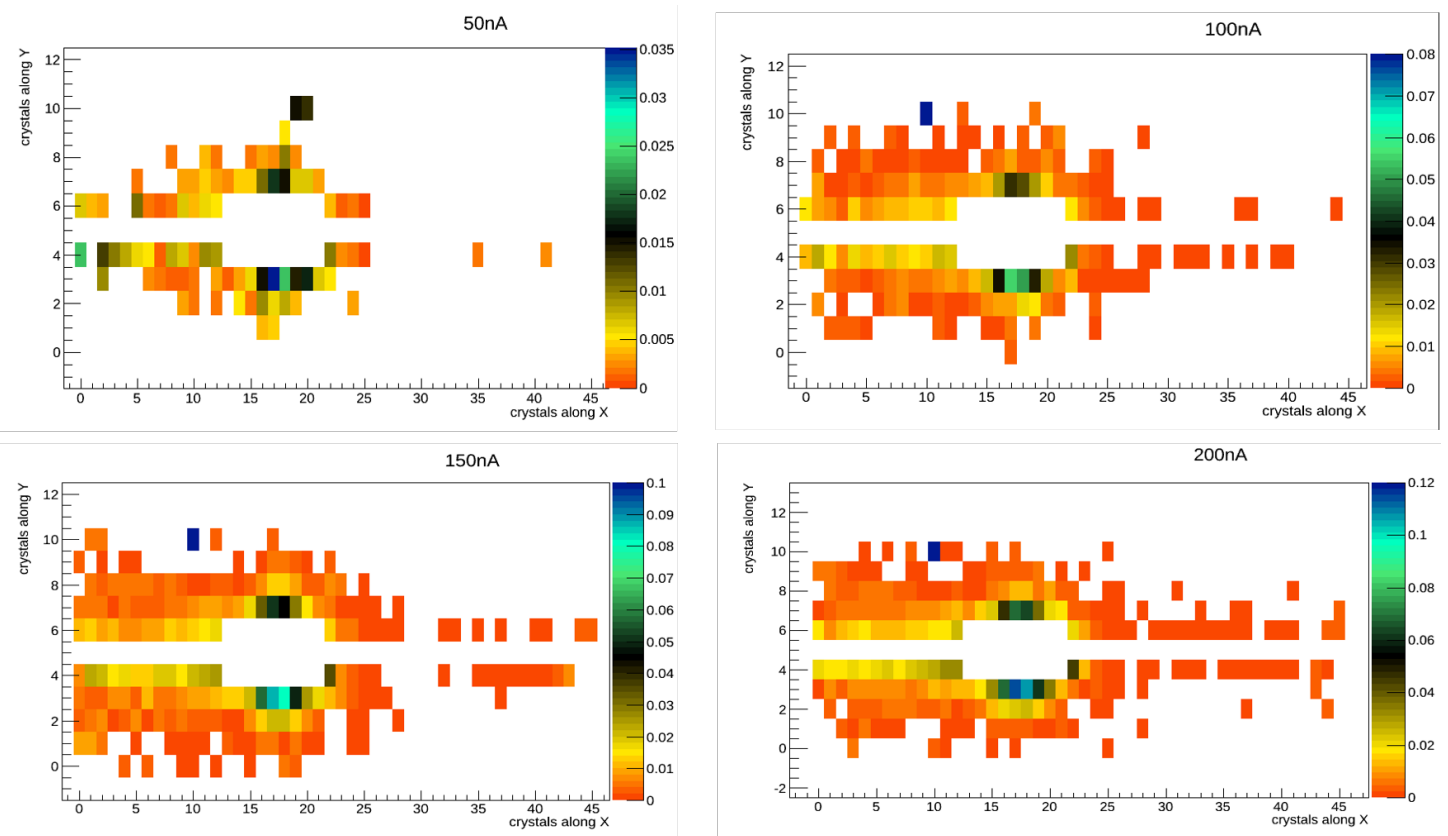

Figure 4.3.2.: Pile-up in the calorimeter as a ratio of the number of signals with two peaks to one peak with a beam current of $50 \mathrm{nA}, 100 \mathrm{nA}, 150 \mathrm{nA}$, and 200 $\mathrm{nA}$. Each $(\mathrm{x}, \mathrm{y})$ bin corresponds to an ECal crystal, $Z$ axis palette is the pile-up ratio.

parameter. It was found that this parameter depends on the type and energy of the particle as well as the top or bottom module of the detectors. The cluster energy is dependent on the properties of a particle and is also correlated with the position of the cluster in the ECal. All these dependencies are parametrized in a combined matching quality factor. The matching algorithm is implemented in the reconstruction software, and for each reconstructed particle track-cluster, the corresponding parameter is stored in the output file along with the other attributes of the reconstructed particle for later use in the data analysis.

The track-cluster matching factor is defined as $n_{\sigma}$ :

$$
n_{\sigma}^{2}=\left(\frac{\mu_{x}(p)-\delta_{x}}{\sigma_{x}(p)}\right)^{2}+\left(\frac{\mu_{y}(p)-\delta_{y}}{\sigma_{y}(p)}\right)^{2}
$$

where $\delta_{x}=\left(x_{\text {track }}-x_{\text {cluster }}\right), \delta_{y}=\left(y_{\text {track }}-y_{\text {cluster }}\right)$, and $\mu, \sigma$ are parameters characterizing the residuals, calculated at the momentum $p$ of the track [120]. The algorithm logic is as follows:

1. For each reconstructed track, all clusters of the same detector module are looped over and $n_{\sigma}$ is calculated.

2. Track-cluster pairs with the smallest $n_{\sigma}$ are coupled. 
3. The energy and position corrections are applied to each cluster based on the particle charge defined in the tracker.

4. Associated tracks and clusters are saved in the ReconParticle class with their matching parameter $n_{\sigma}$.

\subsection{ECal Corrections}

The calorimeter measures the energy that a showering particle deposits in the detector material. The efficiency variations, shower leakage, and the fact that only a part of the total energy becomes the electromagnetic shower, makes it so that the measurement of the energy of a particle is imperfect and needs corrections. Therefore an energy correction function is employed to recover the true initial energy of a given particle. Position correction is applied to improve the cluster position resolution. Another correction is applied for the so called time-walk effect, when the timing of the hits of a cluster are deviating from the seed hit cluster.

\subsubsection{Evaluation of the Energy Correction Function}

To study the position and energy resolution in the calorimeter, the ratio of the reconstructed energy from a measurement in a cluster $\left(E_{\text {rec }}\right)$ to the true energy $\left(E_{\text {true }}\right)$ as given by a GEANT4 MC generator is calculated as a function of the scattering particle's parameters (type, energy and position). The reconstructed energy in the calorimeter depends on the initial energy of the particle and calorimeter properties. The sampling fraction, $\frac{E_{r e c}}{E_{t r u e}}$, is the parameter quantifying this dependence and is calculated based on simulated data.

The different particle types simulated for the calorimeter are electrons, positrons and photons - with energies between $0.3 \mathrm{GeV}$ and $1.1 \mathrm{GeV}(0.1 \mathrm{GeV}$ step-size). This corresponds to the energy range accessible with a $1.05 \mathrm{GeV}$ beam energy. Then, the reconstruction procedure is applied to the output of this simulation as if it were real data and the measured energy is compared to the initial energy of the particle (Etrue).

The energy deposited in the calorimeter is reconstructed using the following threshold criteria:

- > 7.5 MeV deposited per hit (per crystal),

- > $50 \mathrm{MeV}$ energy of the seed hit in the cluster,

- > $100 \mathrm{MeV}$ energy in the cluster in total,

with the seed hit defined as the crystal with the most energy in a cluster. The sampling fraction is calculated in the fiducial region (excluding the edges of the Ecal modules) and is presented in Fig.4.4.1 for electrons, positrons and photons. 


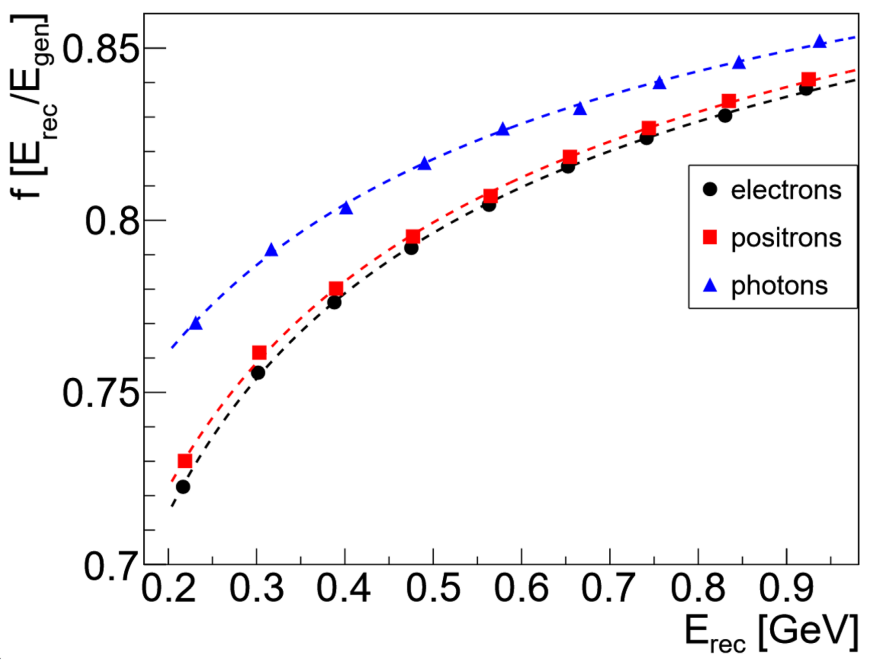

Figure 4.4.1.: Sampling fraction for electrons, photons and positrons in the calorimeter [11]].

The difference between photons, electrons and positrons is explained by the extra material between the target and crystals: the ECal copper thermal shielding and aluminum structure of the Ecal vacuum, as well as the SVT, which are about 0.47 radiation lengths all together. Moreover, because of the magnetic field the incident angles and positions have an energy-dependent impact on the sampling fraction.

\section{Edge Effect and Correlation Studies}

We extracted the energy correction as a function of the shower distance from the edge of the calorimeter. In the central region, the sampling fraction is practically constant, but this changes near the edges; the efficiency of reconstructing the true energy of the incident particle drops, showing a strong correlation with the cluster position, see Fig. 4.4.2. When using experimental data, the position is defined from the kinematics measured in the tracker. This same study was done for positrons and photons. The position resolution for the $\mathrm{x}$ and $\mathrm{y}$ coordinates of a cluster obtained from these studies is about $2 \mathrm{~mm}$ for $1 \mathrm{GeV}$ particles [111].

\subsection{Calibration of the ECal}

To calibrate the calorimeter at small, medium and nearly full beam energies, various processes were used. The initial calibration of the gains was done with cosmic rays. Then, Moller electrons were used to improve the gain coefficients, wide-angle Bremsstrahlung events were used to adjust the sampling fraction for particles with 


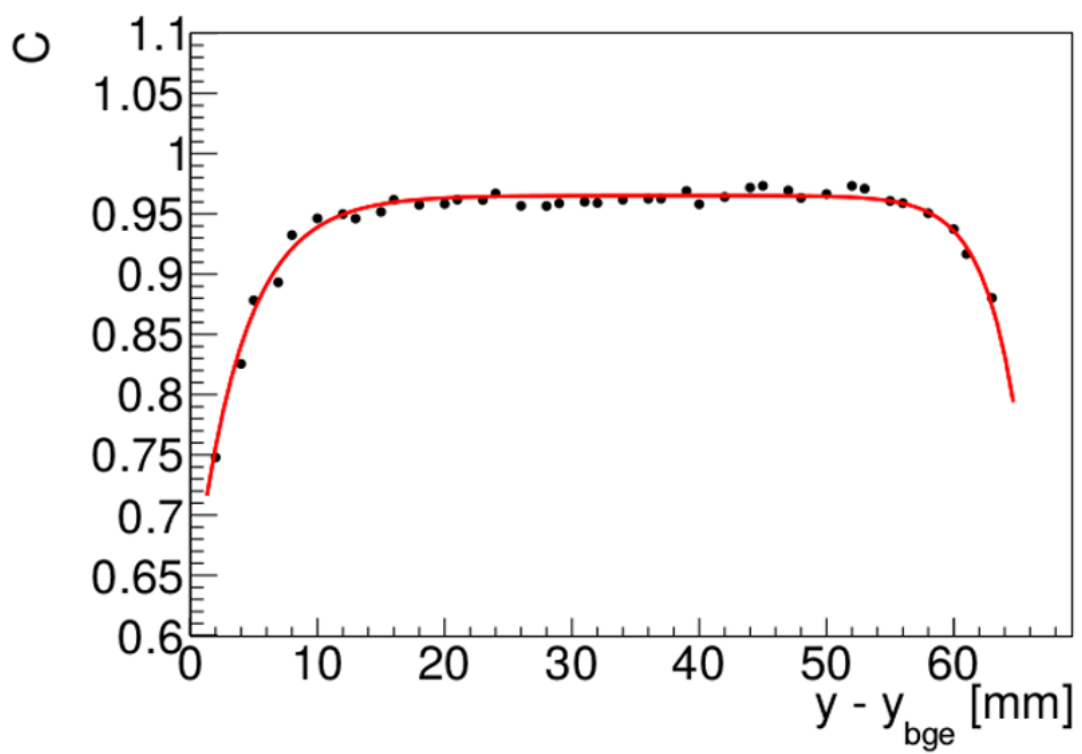

Figure 4.4.2.: Energy drop for an electron versus the vertical position of the clusters relative to the cluster nearest to the beam gap edge [111].

lower energies and full energy electrons, produced in small angle elastic scattering, are exploited to perform calibration at high energies.

\subsubsection{Calibration with cosmic rays}

Cosmic rays enter the calorimeter almost vertically, but leave a signal too small to trigger. Plastic scintillator paddles were placed over and under the detector, in order to trigger the read-out of the ECal. From all tracks formed in the ECal, we want to select the vertical ones, in order to measure the average homogenous energy loss in each crystal. From simulations, this energy deposit was found to be $\approx 18.3 \mathrm{MeV}$ [111]. Cosmic ray runs were about 60 hours long. Response from all crystals were fitted to obtain the gains of the 442 channels.

To identify the cosmic clusters amongst the crystals triggered for a given cosmic event in the top and bottom ECal modules, an algorithm was developed. The pulse distribution of each hit in the track was analyzed and the energy and timing information was used to define the timing offsets of crystals, the time resolution and the energy resolution.

From the final analysis, the relative energy resolution was found to be about $\frac{8 \%}{\sqrt{E(G e V)}}$ [11]. 


\section{The Energy Threshold of a Cosmic Hit}

The energies deposited by cosmic particles are very close to the thresholds for signalnoise separation. With the APDs used in the 2012 test run, the discrimination between noise and signal at these energies was not possible. The APDs were upgraded from $5 \times 5 \mathrm{~mm}^{2}$ to $10 \times 10 \mathrm{~mm}^{2}$ for the 2015 run to yield 4 times more light and make possible a calibration at such low energies.

Different threshold settings (10 FADC,11 FADC,12 FADC and 13 FADC) were tested for the discrimination of a cosmic signal from a random hit pattern. The 10, 11 FADC thresholds were found to be too close to the background noise and tended to generate a lot of accidental hits around the clusters of the true particle, thereby, contaminating the crystals hit-fields that would be analyzed in the cosmic-clustering algorithm. Another issue with the low threshold is the poor noise-signal separation in the pulse shape which makes a fit to a signal pulse rather uncharacteristic. Finally, the 12 FADC was chosen as the best compromise between the maximum possible coverage at low energies and reliable results.

\section{Cosmic-Track Clustering Algorithm for ECal}

To perform the calibration with cosmic rays, a specific clustering algorithm in the calorimeter modules had to be developed in order to find the cosmic trails. The typical trace of a cosmic particle passing through the detector would ideally induce a signal in every layer of the calorimeter leaving a perfectly vertical or slightly inclined line of triggered crystals as a signature (because of the trigger configuration for cosmics). This pattern was used to develop a clustering algorithm for cosmic tracks across the calorimeter crystals . Typical patterns for a few cosmic events are presented in Fig. 4.5.1. This map is passed to the clustering algorithm to sort out the clusters of hits coming from the cosmic ray events. The algorithm consists of the following steps:

- From the hit map, a cluster map is created, consisting of collections of more than one consecutive hits.

- The largest cluster in one of the ECal module is selected as an origin around which the cosmic-track candidate is formed. For that candidate, the $\mathrm{O}_{i}(\mathrm{x}, \mathrm{y})$ center of $i$ - th cluster is defined as an arithmetical average of column numbers and layer numbers of the collection of its hits. The columns and layers of crystals are comprised within $\{0-46\}$ and $\{0-10\}$ respectively. The correlated clusters are found through reconstruction of a line across the cluster map passing through the origin-cluster and other clusters. The inclination angle of a line, passing through the $\mathrm{O}_{0}^{c}$ center of the origin-cluster and $\mathrm{C}_{j}(\mathrm{x}, \mathrm{y})$ of the $j$-th crystal of the $i$-th cluster considered, is calculated. Using the vertical or nearly vertical requirement forced by the trigger, it is asked that $\Delta \mathrm{x}=\mathrm{x}_{O}\left(\mathrm{y}_{C_{j}}\right)-\mathrm{x}_{C_{j}}<5$ to filter out uncorrelated clusters. 


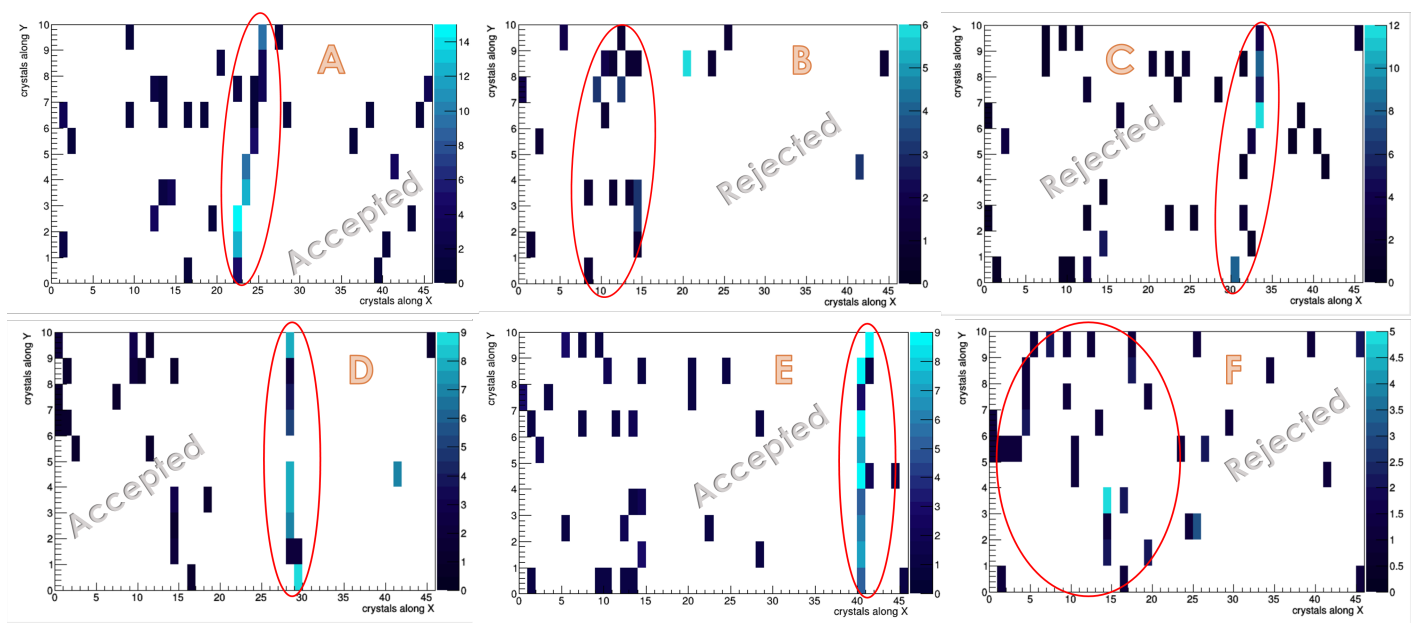

Figure 4.5.1.: A typical map of crystals with hits for arbitrary events. The $X$ and $Y$ axes represent the columns and layers (rows) of the calorimeter respectively. Each bin on the plot indicates a hit above threshold in the crystal at the $\mathrm{x}$-th column and the y-th layer. The $Z$ axis, indicated as a color palette on the right of each plot, denotes the number of hits in a crystal per event.

- At this stage, the identified cosmic-track candidates are analyzed further based on their geometrical configuration. From these candidates, only the tracks with a signal in at least 5 layers of the ECal are selected.

- Tracks with three or more consecutive crystals in a layer are excluded (see plots B and F in Fig. 4.5.1 ).

- Surviving candidates are checked against a zigzagging pattern (plot $\mathrm{C}$ in Fig. 4.5.1).

\section{Timing Offsets and Resolution Studies}

The timing of the signal formed in the crystals is extracted from the pulse information sampled by the FADC counters every 4 nanoseconds. This allows for the estimation of the timing characteristics of the detector and perform for a timing calibration and the calculation of the offsets between each crystal.

For this calculation, the cosmic tracks found by the cosmic-clustering algorithm are considered (like the A, D, E tracks in Fig. 4.5.1). The pulse of each hit is fit with the function of the form of eq.(4.3.2), see Fig.4.5.2. The maximum of the fit and the peak position are compiled for all hits of a track. This is repeated for all events which qualify as a cosmic track. The peak position distribution and the height are fitted with a Gaussian function to extract the timing offsets, the time resolution and energy resolution of each crystal. In Fig. 4.5.3, the offsets at different threshold settings are shown. The $X$ and $Y$ axes represent the crystal position ID as the $x_{i}$-th column of the $y_{i}$-th layer for the i-th crystal-cell. The $Z$ axis is the value of the offset 

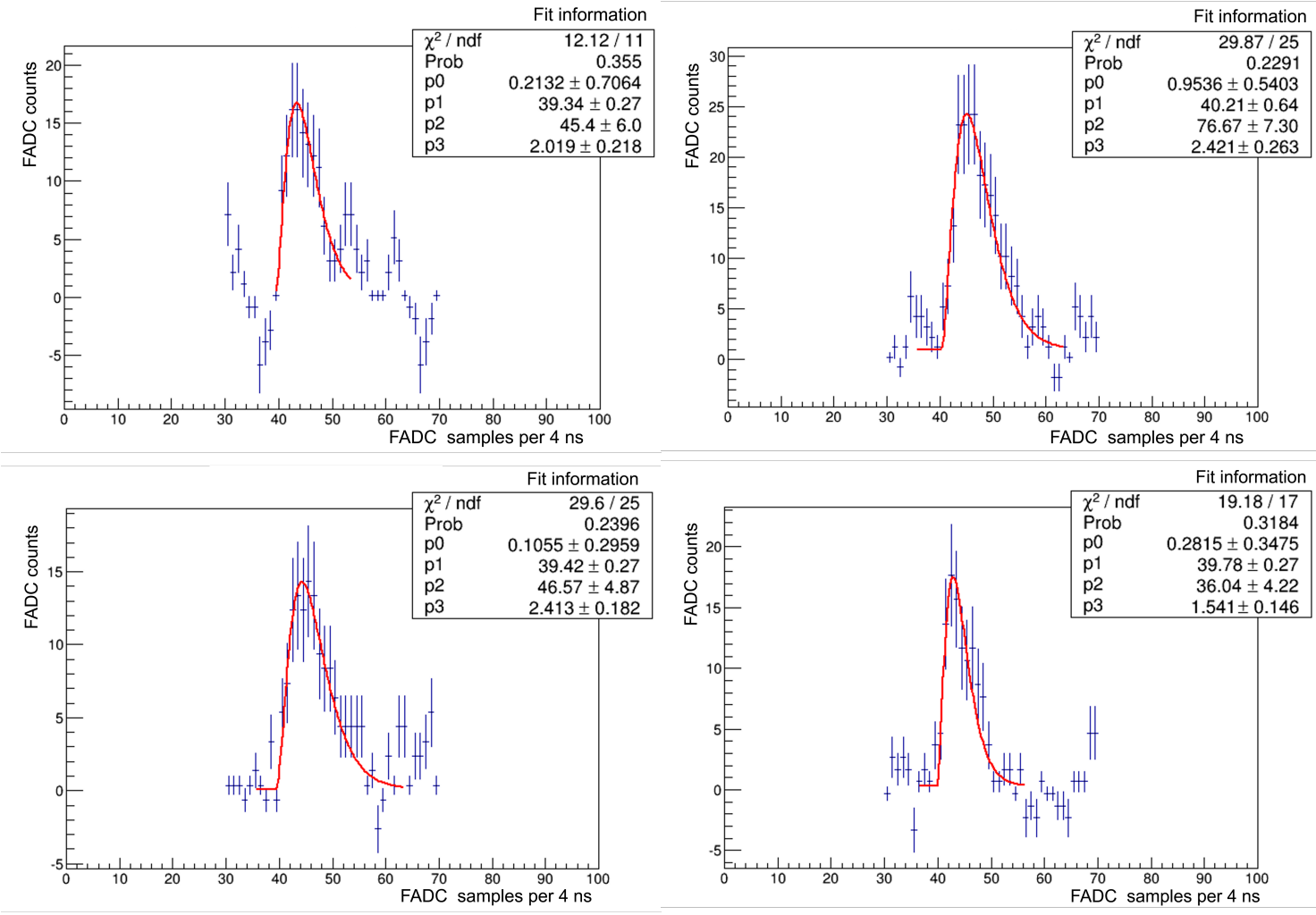

Figure 4.5.2.: Fit to the hit pulse distribution from a track. The fit parameters $\mathrm{p} 0, \mathrm{p} 1, \mathrm{p} 2, \mathrm{p} 3$ correspond to $\mathrm{P}, \mathrm{t}_{0}, \mathrm{~A}, \tau$ in eq.(4.3.2) respectively.

for each crystal . The results are more or less homogeneous across the crystal module except for the brown group of crystals that show a significantly different offset. This is due to the cables connecting this group of crystals which are of different length than for the rest of the detector.

From the same fits, we obtain time resolution for each crystal presented in Fig. 4.5.4. The average resolution value across the calorimeter is about $\sim 4 \mathrm{~ns}$. This low resolution is due to the small signal.

\section{Energy Resolution Study}

The energy resolution of the calorimeter was analyzed along with the timing study with the cosmic ray data from 2014 and early 2015. The cosmic track searching algorithm was used to find the tracks with the 10 FADC threshold setting to maximize the statistical outcome. The distributions of the pulse integrals are fitted with Gaussian functions and the width of the resulting energies is considered as an estimate of the energy resolution. In Fig. 4.5.5, the map of crystals with the relative energy resolution as the $Z$ axis value is shown. The results with the integrated signal are obtained from the energy of cosmic hit within a 10 samples window around the 


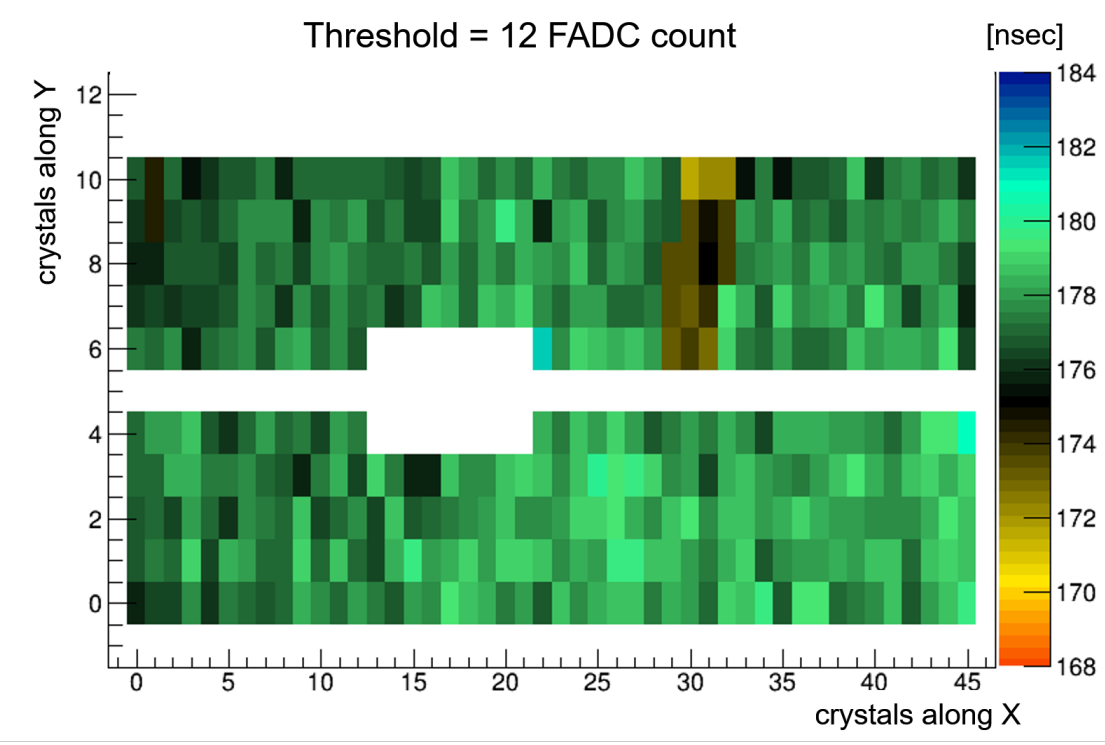

Figure 4.5.3.: Timing offsets from cosmics for the 12 FADC threshold setting.

peak pulse. Crystals on the edges of the ECal are not statistically significant and deviate from the Gaussian width.

\subsubsection{Energy Calibration with Beam Data}

Calibrations for higher energies are done with beam data using Moller electrons and electron-positron pairs from wide angle Bremsstrahlung events [111].

\section{Calibration with Elastically-Scattered Electrons}

Beam electrons scattered elastically at small angles by the nuclei of the target carry almost all of the energy of the beam and are detected in the ECal within $\approx \pm 1 \%$ of their initial energy (1.056 GeV for the current analysis). Events for this calibration were selected such that:

1. $E_{\text {seed }}>0.6 \times E_{\text {cluster }}$ - meaning that the seed hit contribution in the total energy of the cluster is more than $60 \%$ of the total energy of the cluster.

2. $E_{\text {seed }}>450 \mathrm{MeV}$ - the seed hit energy is at least $450 \mathrm{MeV}$ for the $1.056 \mathrm{GeV}$ beam.

The clusters were used to calibrate every single crystal that they had as their seed hit. Thus, crystals in the geometrical acceptance region for full energy electrons (366 crystals) were calibrated in two iterations of this calibration procedure, to include as many crystals of the ECal as possible. This was enough to have the gains of each crystal to be stable within a $1 \%$ error. The energy correction function was then applied to the clusters energies. 


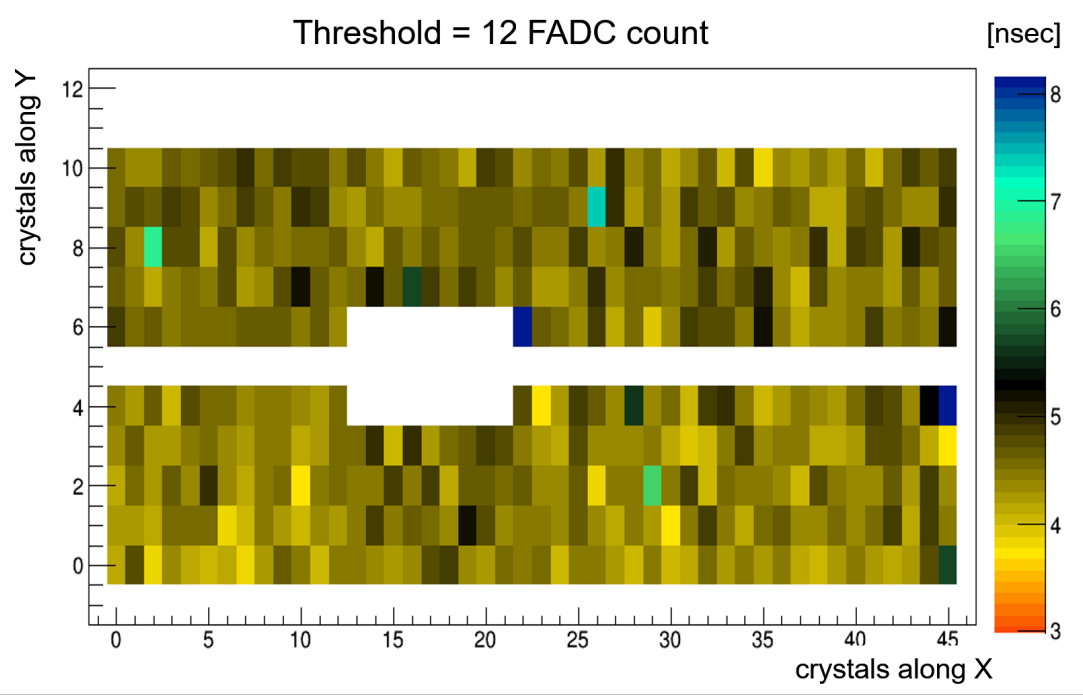

Figure 4.5.4.: Time resolution from cosmics for the 12 FADC setting for the signal threshold.

\section{Calibration Using Wide-Angle Bremsstrahlung Events}

One of the largest background processes for the HPS is the so-called wide-angle Bremsstrahlung, where the target scattered electron converts to a $e^{+} e^{-}$pair interacting with the SVT layers. This leaves only one matching electron track in the SVT, but forms two clusters in the ECal. The total energy of these two clusters is expected to be equal to the beam energy from the trigger requirements. The calibration with wide-angle Bremsstrahlung is detailed in [111].

\subsubsection{Studying the ECal Time Resolution with the Beam Data}

The time resolution study with cosmic rays showed time resolution roughly similar to the FADC sampling resolution. One of the problems with cosmics is the low amplitude signal, which makes the signal-noise separation unclear and distorts the pulse shape. Beam events have significantly higher energy, therefore the light yield in the crystals is larger and the pulse shape is better defined improving the fits and eventually the final end results for each crystal.

The timing is studied using the reconstructed cluster information and separately the raw data pulse information in the crystals of the cluster. The results are compared. For the raw data analysis, the threshold was set to 100 FADC above the pedestal value.

The signal for each crystal in the cluster is fitted with eq.(4.3.2), illustrated in Fig. 4.5.6. The peak position from these fits is compiled in a histogram. This is equivalent to compiling the time of the reconstructed cluster crystals, which is 


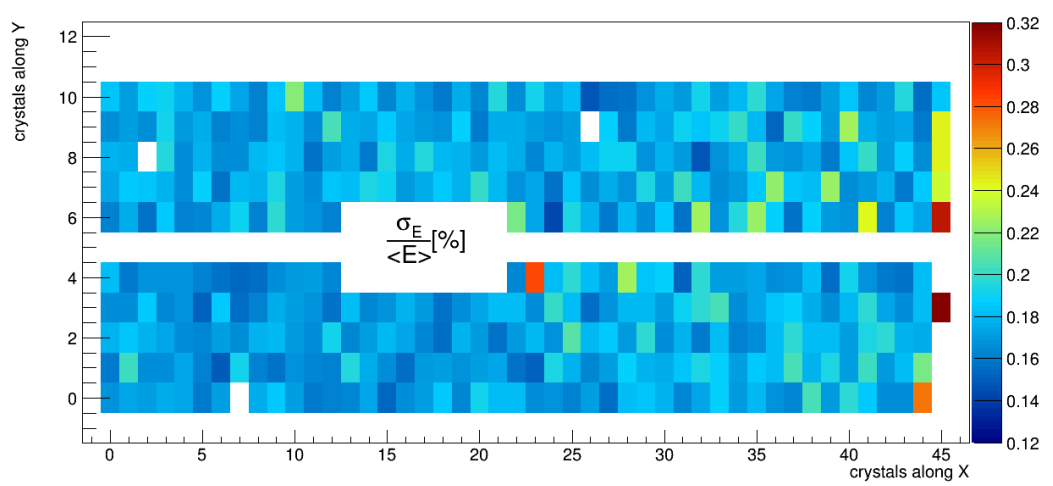

Figure 4.5.5.: Relative energy resolution in the calorimeter with the integral signal for cosmics rays, using the 10 FADC setting for the signal threshold.

defined from the timing of its seed hit. The energy threshold implemented for a cluster is $>200 \mathrm{MeV}$. For each crystal, we obtain two distributions of its timing, one from the reconstructed time of the cluster and another one from the fits to the pulse of each crystal in the cluster. Next, these distributions are fitted with Gaussian functions. The timing map of each crystal obtained from these two methods is shown in Fig. 4.5.7. The difference of the average values is due to a nominal shift in the recorded FADC samples. Both methods show similar behavior. The time resolution estimations are similar too (see Fig. 4.5.8), showing a slightly more stable value across the detector from the reconstructed cluster-hit timing than from raw hit data. This difference can be due to minor implementation differences (energy FADC equivalence). In the analysis with raw hit information, there is a contribution from the crystals with smaller energies and from crystals with uncorrelated hits. A finer precision can be obtained from coupling the time measured by the FADC with the RF signal time of the beam (see next section).

\section{Timing and Time Resolution with RF Signal}

In order to have a more accurate measurement of the time resolution of the ECal and of the timing offsets for each crystal, the RF signal of the beam is used as a reference to match with the FADC signal. The RF signal has a $499 \mathrm{MHz}$ frequency (2.004008 ns), sampled for every 80 beam bunch, whereas the FADC signal is sampled at $250 \mathrm{MHz}(4 \mathrm{~ns})$. With the RF pulse, the timing of the signal in the crystal can be fine-tuned to a much better precision than with the FADC clock. As a first step, to find the time reference value, the RF signal distribution was fitted. The stability of this value defines the precision of the method. The attempts were focused on taking into account at least three points in the rise of the RF distribution, in order to have some fit power with a two parameter polynomial as the fit-function. The time at $\sim 70 \%$ of the maximum of the RF signal was used as a reference for the event. The difference of the hit timing measured in the FADC and the reference RF 

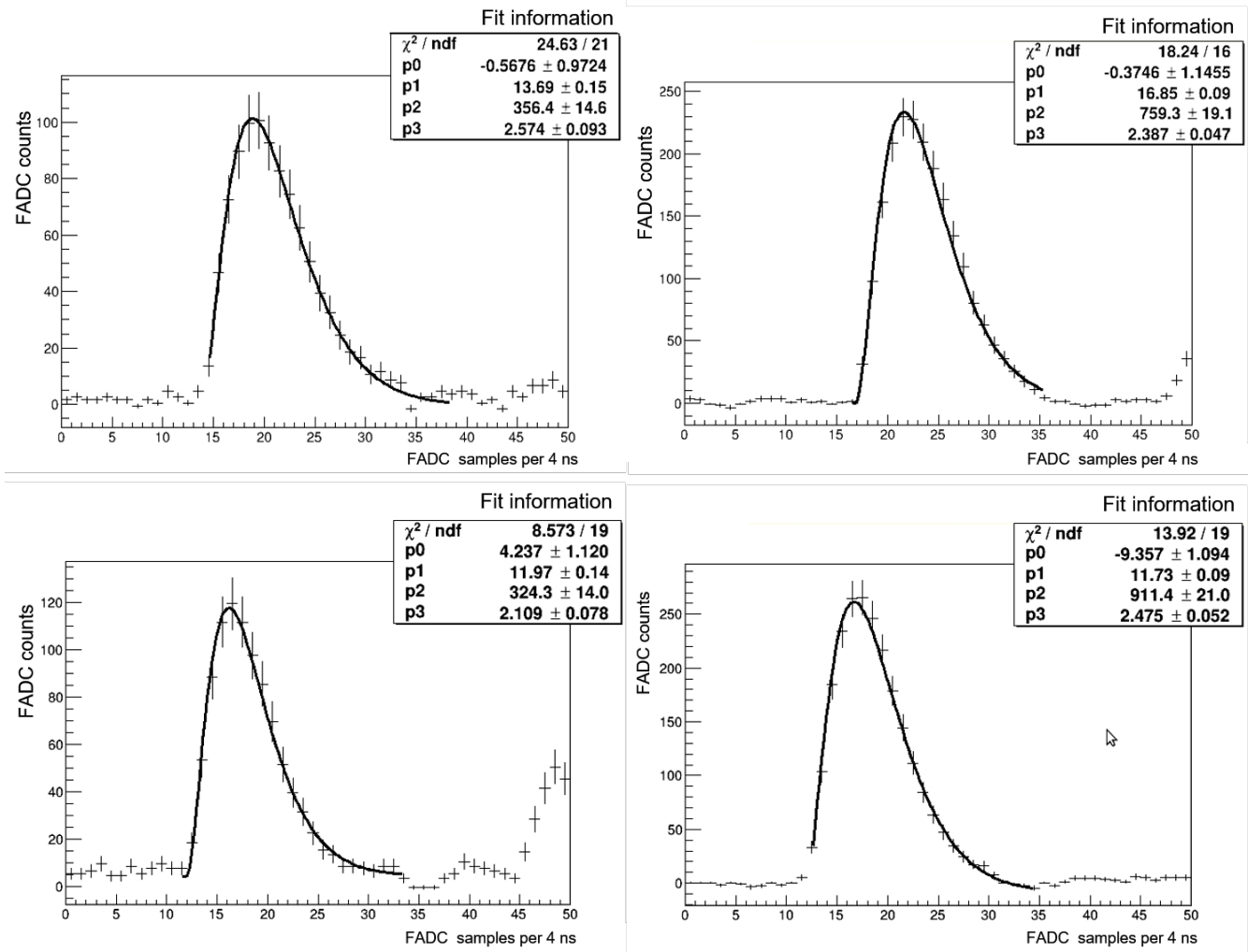

Figure 4.5.6.: Fit to the hit-signal of a cluster using raw pulse data, 100 FADC above pedestal value with eq.(4.3.2).

time is folded relative to the reference RF time, by taking the modulo 2.004 nsec. The result is compiled and fitted with a Gaussian. Widths for each crystal are shown in Fig. 4.5.10, showing an average $\sim 0.4 \mathrm{~ns}$ time resolution all along the calorimeter.

Similar analysis was carried out in [121] through multiple iterations obtaining a finer resolution depending on the hit energy.

\subsection{Conclusion}

Different aspects of the ECal performance were discussed in this chapter. The position corrections for the ECal from the survey required to modify the geometrical model used in the simulations. These corrections were applied and the implementation of the ECal geometry in the detector simulation package was updated, allowing for geometrical corrections of the ECal modules independent from each other. This will help to apply corrections of the ECal position from regular surveys for the future runs as well.

The event reconstruction, including hit reconstruction in the SVT and the ECal, track finding and cluster-track matching algorithms were briefly introduced. The 

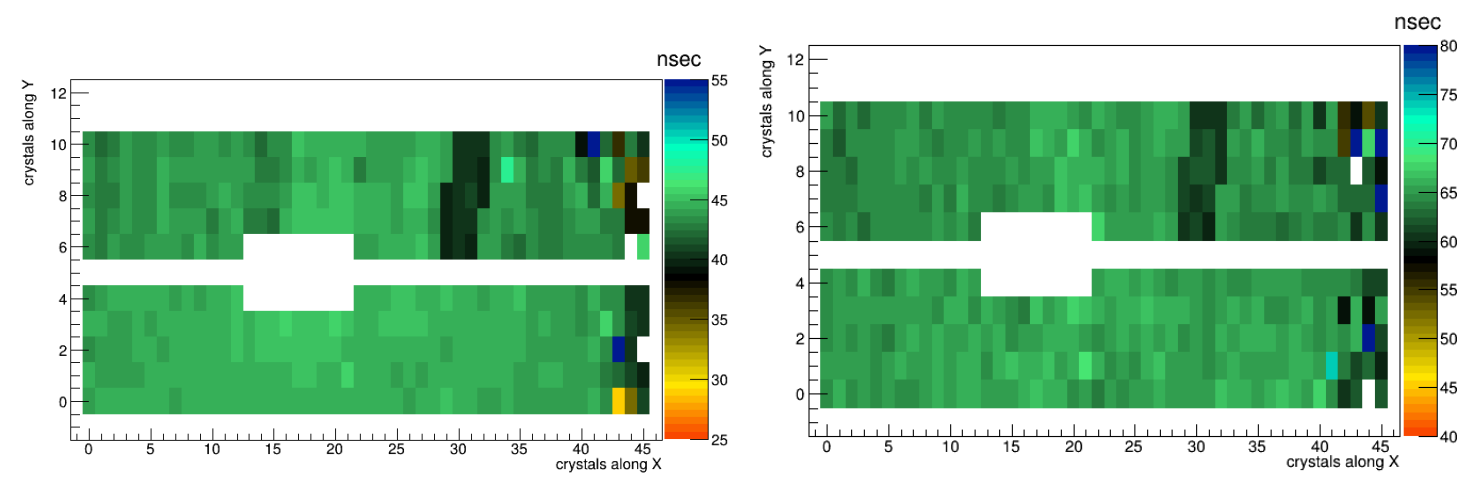

Figure 4.5.7.: The timing map of the crystals with reconstructed cluster information (left) and from the hit raw data information (right).
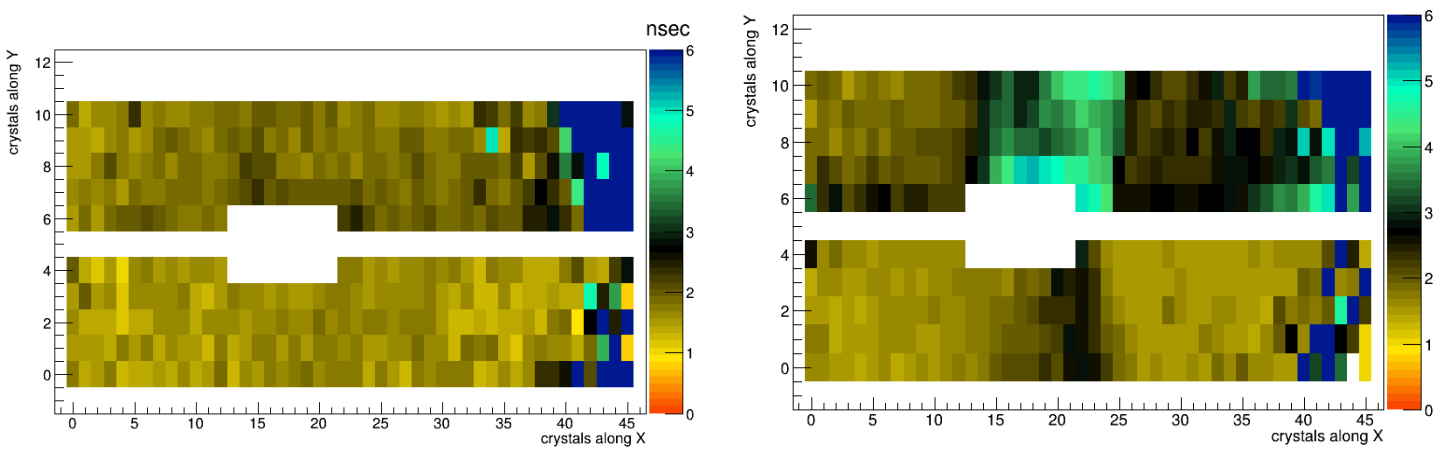

Figure 4.5.8.: The timing map of the crystals with the reconstructed cluster information (left) and from the hit raw data information (right).

optimal size of the window for FADC pulses was established, in order to provide the full information of the signal. In a related study, the pile-up evaluation in the ECal at different beam current showed a small $(<5 \%)$ pile-up contamination even at the highest, $200 \mathrm{nA}$, current values. The timing and the time resolutions were studied with cosmic rays and beam data. For cosmics, a track-searching algorithm was developed and the time resolution at such low energies is about 4 ns. For higher energies, the timing and time resolution are obtained with the beam data. Results of these studies showed a less than $1 \mathrm{~ns}$ time resolution for the crystals in the calorimeter. The most precise time resolution was found using the time of RF pulses as a reference clock. The average time resolution obtained from this study is about $0.4 \mathrm{~ns}$ for $1 \mathrm{GeV}$ electrons. 

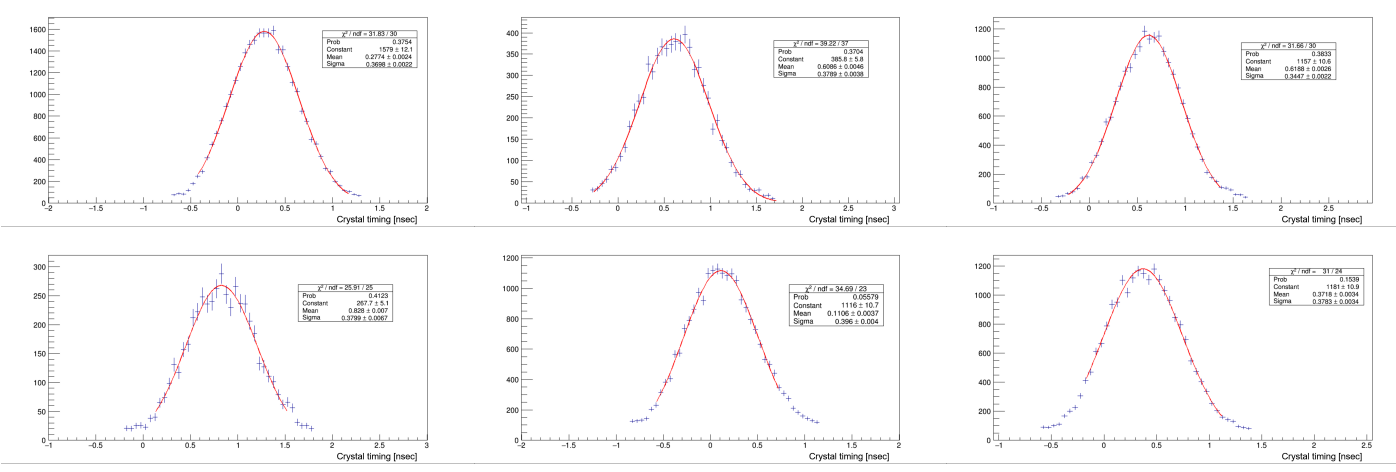

Figure 4.5.9.: Gaussian fit to the distribution of the FADC timing relative to the $\mathrm{RF}$ reference time for several crystals.

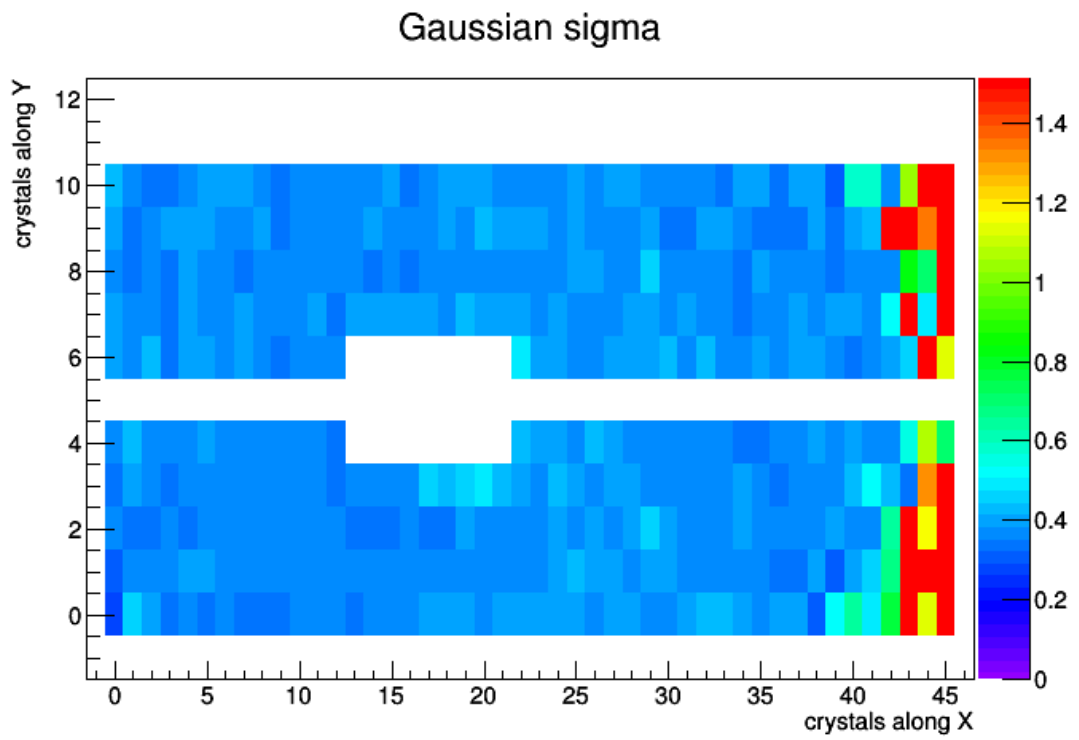

Figure 4.5.10.: Time resolution for each crystal in the calorimeter using the reference RF time. 



\section{Background Simulations}

\subsection{Introduction}

In this chapter, I will review the important backgrounds from QED processes that can be affecting the HPS experiment. For our bump hunt analysis, it is crucial to understand these backgrounds. They consist of $e^{-} e^{-} e^{+}$final states produced in QED processes. There are three main sources of such QED processes for HPS: the wide angle bremsstrahlung (WAB) that converts a $\gamma$ to $e^{+} e^{-}$(Fig. 5.2.1), multiple Coulomb scattering in the target and the prompt single vertex radiative process (Fig. 5.3.1). These backgrounds are studied with simulated data samples. To produce these samples, we use the following event generators:

1. bremsstrahlung photons from the target - MadGraph/MadEvent4 [116], then GEANT4 for the conversion of the photon into $e^{-} e^{+}$,

2. multiple Coulomb scatterings - EGS5 [122], GEANT4 [110].

3. single vertex prompt radiative process - MadGraph5 [117], MadGraph/MadEvent4 [116], Monte Carlo numerical integration based pseudo ${ }^{1}$ generator (BVMC as for Beranek-Vanderhaeghen Monte-Carlo) by [123],

At the output of the event generator, these generated data (except the samples of the BVMC, since it uses weighted events) are mixed with the simulation of other processes, such as hadronic processes, and run through the read-out simulation and reconstruction codes to obtain a fully simulated sample. We have more than one generator for each physics channel to ensure that we can obtain robust results. An important focus of my work was on the BVMC event generator as a discrepancy found between the single vertex radiative processes calculated with MadGraph4, MadGraph5 and most importantly, data.

\subsection{WAB and Multiple Coulomb Scattering Backgrounds}

The high intensity beam current, in combination with the detector configuration with very forward acceptance, leads to important electron and photon fluxes in the

\footnotetext{
${ }^{1}$ It is a flat event generator which ascribes a "weight" to each event.
} 
detector coming from multiple Coulomb scattering and bremsstrahlung processes. Significant contamination of our signal is coming from the bremsstrahlung photons, formed mostly in the target and converted into electron-positron pairs in the target or in the layers of the tracker. Illustrations of such processes are presented in Fig. 5.2.1. Multiple Coulomb scattering and bremsstrahlung real photon production can be reduced by using thinner targets. However, to maintain the dark photon production rate, the beam intensity has to be increased at the same time [63]. This leads to practical issues, like local overheating of the target, already discussed in sec. 3.3.3. These processes were simulated with MadGraph4 for the bremsstrahlung photons in the target. The conversion to electron-positron pair is simulated with GEANT4. EGS5 and GEANT4 are used for the simulation of multiple Coulomb scattering process [63].

\subsection{Prompt Single Vertex QED Background}

The prompt single vertex QED background is formed of the Bethe-Heitler tridents and radiative processes, presented in Fig. 5.3.1. Note that in both cases, the initial scattered electron can be detected as one of the decay electron, as shown in Fig. 5.3.2.

The background coming from the Bethe-Heitler process dominates over both the dark photon production and radiative trident. However, the kinematics of the pair formation in the Bethe-Heitler process is such that it produces mostly low energy electron-positron pairs [63]. This is due to the Bethe-Heitler processes having a forward directional singularity. It favors pair production with asymmetric energies, where one of the electrons is soft and bent at large angle, while the other one is scattered forward at small angles, as illustrated in Fig. 5.3.2. These kinematical features can be exploited to reduce the contribution of the Bethe-Heitler pairs in the final sample of $\gamma^{\prime}$ events.

The radiative processes such as the one on the right of Fig. 5.3.1, mimic the HPS signal. Therefore, the invariant mass window containing the $\gamma^{\prime}$ resonance peak, will include events coming from such QED radiative tridents. Contrary to Bethe-Heitler, the radiative tridents pair production process is more likely to produce energetic lepton pairs [63]. The similarity between dark photon and radiative tridents kinematics allows one to use its measurement to draw conclusions about dark photon production kinematics.

The dominant contributions in different energy regions along with the dark photon production can be summarized in a plot of the electron energy versus the positron energy obtained from real data, see Fig. 5.3.3. 


\subsubsection{Radiative and Bethe-Heitler Trident Simulations}

To study the radiative and Bethe-Heitler processes, we used a Monte-Carlo numerical simulation, based on the theoretical calculations by Beranek and Vanderhaeghen [124, 123, 125], along with MadGraph4/5. The results of both approaches will be compared. In addition, we will investigate the different contributions of the radiative and Bethe-Heilter diagrams, separating the direct terms (top diagrams in Fig. 5.3.4 and Fig. 5.3.5) and exchange terms (bottom diagrams in Fig. 5.3.4 and Fig. 5.3.5).

\subsubsection{MadGraph5 Implementation}

MadGraph5 is a software, which calculates cross-sections within both SM and beyond SM phenomenology [117]. The program is meant for collider simulations, but can be configured for fixed target experiments as well. For the HPS background simulation, the cross-sections are calculated for the process $e^{-} \mathrm{Z} \rightarrow e^{-} e^{+} e^{-} \mathrm{Z}$ where $\mathrm{Z}$ denotes the target nucleus. Calculations are carried out separately for the radiative tridents (Rad), for Bethe-Heitler radiation $(\mathrm{BH})$ and for $\mathrm{Rad}+\mathrm{BH}$ combined. The kinematical cuts and acceptance region implemented for the MadGraph5 calculations are presented in Tab. 5.3.1.

\begin{tabular}{|c|c|}
\hline MG5 & Kinematic conditions \\
\hline \hline$E_{\text {beam }}$ & $1.056 \mathrm{GeV}$ \\
\hline$E_{\text {min }}^{e^{ \pm}}$ & $>0.05 \mathrm{GeV}$ \\
\hline$E_{\text {sum }}$ & $>0.5 \mathrm{GeV}$ \\
\hline$|\varphi|$ & $<\pi$ \\
\hline$|\theta|$ & $>10[\mathrm{mrad}]$ \\
\hline
\end{tabular}

Table 5.3.1.: Fiducial region used with MadGraph5, where $E_{\text {beam }}$ is the beam energy, $E_{m i n}^{e^{ \pm}}$is the minimum energy of the electron and positron, $E_{\text {sum }}$ is the energy sum of the electron and positron pair, $\phi$ and $\theta$ are respectively the azimuthal and the polar angle of the electron and positron in the laboratory frame.

\subsubsection{The Theoretical Model for Monte Carlo Calculations}

In $[125,123,124]$, a theoretical framework has been developed to calculate the crosssection for the diagrams shown in Fig. 5.3.4 and Fig. 5.3.5.

Assuming that the contributions from double virtual Compton scattering (VVCS) processes, shown in Fig. 5.3.6, can be neglected in the case of a heavy target, the 
differential cross-section can be calculated as [125]

$$
\begin{aligned}
d \sigma= & \frac{1}{4 \sqrt{(k \cdot p)^{2}-m^{2} M^{2}}}(2 \pi)^{4} \delta^{(4)}\left(k+p-k^{\prime}-p^{\prime}-l_{-} l_{+}\right) \\
& \times \frac{d^{3} \overrightarrow{k^{\prime}}}{(2 \pi)^{3} 2 E_{e}^{\prime}} \frac{d^{3} \overrightarrow{p^{\prime}}}{(2 \pi)^{3} 2 E_{p}^{\prime}} \frac{d^{3} \vec{l}_{-}}{(2 \pi)^{3} 2 E_{-}} \frac{d^{3} \overrightarrow{l_{+}}}{(2 \pi)^{3} 2 E_{+}}|\overline{\mathrm{M}}|^{2},
\end{aligned}
$$

where $k$ and $k^{\prime}$ are the incident electron four-momenta before and after interaction, $p$ and $p^{\prime}$ represent the four-momenta of the nucleus, and $l_{+}$and $l_{-}$correspond to the four-momenta of the produced lepton pair. $\bar{M}$ is the amplitude of the process, and $m$ and $M$ stand for the mass of the electron and the mass of the target nucleus. The differential cross-section integrated over the fiducial region can, then, be expressed as (eq.(11) in [125]):

$$
\Delta \sigma_{\gamma} \propto\left|M_{D, \gamma^{\star}}^{T L}+M_{D, \gamma^{\star}}^{S L}-M_{X, \gamma^{\star}}^{T L}-M_{X, \gamma^{\star}}^{S L}\right|^{2} .
$$

Here, TL and SL notation refer to the timelike and spacelike Feynman diagrams, D and $\mathrm{X}$ denote direct and exchange diagrams respectively. Detailed descriptions of the cross-section computation along with the formulas calculating each amplitude in eq.(5.3.2) are given in [125, 124].

Based on the calculations described above, the authors have developed a Monte Carlo numerical simulation implemented in $\mathrm{C}$ programming language. Their background estimation for the HPS signal, was presented in [123]. We used the same code to compare with the MadGraph4/5 event generator results.

\subsubsection{The Monte Carlo Simulation}

The differential cross-section of $e(k) Z(p) \rightarrow e\left(k^{\prime}\right) Z\left(p^{\prime}\right) e^{-}\left(l_{e^{-}}\right) e^{+}\left(l_{e^{+}}\right)$can be described by two sets of variables, shown in Tab.5.3.2. Each variable is uniformly generated and the 8-fold differential cross-section, corresponding to the 8 independent kinematical variables of the process, is calculated from eq.(5.3.1), to produce events $^{2}$. The distribution for a variable is obtained by weighting each generated value by the differential cross section and integrated over the remaining variables within the acceptance of HPS detector.

\section{The Initial Estimates of QED Background}

We first attempted to recover the results of [125]. We found that the simulations in [125] were performed for a positron beam, due to, with all due respect to the

\footnotetext{
${ }^{2}$ In contrary to MadGraph4/5 the output event of BVMC generator is not a standard event. One has to apply corresponding Jacobian transformation for each variable that is not a part of 8 dimensional phase space used to calculate the cross section.
} 


\begin{tabular}{cccc}
\hline Set 1 & Energy & out-of-plane angle & horizontal angle \\
\hline \hline Positron & $E_{e+}$ & $\vartheta_{e^{+}}$ & $\varphi_{e^{+}}$ \\
\hline Electron & $E_{e^{-}}$ & $\vartheta_{e^{-}}$ & $\varphi_{e^{-}}$ \\
\hline Beam electron & & $\vartheta_{e^{-}}^{\prime}$ & $\varphi_{e^{-}}^{\prime}$ \\
\hline
\end{tabular}

\begin{tabular}{ccccc}
\hline Set 2 & Energy & out-of-plane angle & Horizontal angle & Invariant mass \\
\hline \hline Positron & $E_{e+}$ & $\vartheta_{e^{+}}$ & $\varphi_{e^{+}}$ & \\
\hline Electron & & $\vartheta_{e^{-}}$ & $\varphi_{e^{-}}$ & $m_{e^{+} e^{-}}$ \\
\hline Beam electron & & $\vartheta_{e^{-}}^{\prime}$ & $\varphi_{e^{-}}^{\prime}$ & \\
\hline
\end{tabular}

Table 5.3.2.: The sets of independent kinematic variables used for the Monte Carlo simulations.

authors, an inaccurate sign input implementation in the code. Otherwise, the results published in [125] were reproduced in the same fiducial region and compared with the correct setting. As seen from Fig. 5.3.7, the outcome of the calculation differs by $\sim 20 \%$, depending if one considers a positron or an electron beam. The fiducial region used in this calculation is summarized in Tab. 5.3.3.

\begin{tabular}{|c|c|}
\hline BVMC & Kinematical Conditions \\
\hline \hline$E_{\text {beam }}$ & $1.056 \mathrm{GeV}$ \\
\hline$E_{\text {min }}^{e^{ \pm}}$ & $>0.5 \mathrm{GeV}$ \\
\hline$E_{\text {sum }}$ & $>1.0 \mathrm{GeV}$ \\
\hline$\left|\varphi_{\text {hor }}\right|$ & $<50[\mathrm{mrad}]$ \\
\hline$\left|\theta_{\text {out }}\right|$ & {$[-60,-15] \cup[15,60][\mathrm{mrad}]$} \\
\hline
\end{tabular}

Table 5.3.3.: Fiducial region used in [123] and for our results, reproducing the calculations of [123], presented in Fig. 5.3.7.

\section{Estimating the HPS Acceptance}

We also noticed that the calculations published in [125] corresponded to a rather small kinematic region, corresponding to Tab. 5.3.3, while the HPS acceptance covers quite a broader interval of energies and angles (see Tab. 5.3.4). To obtain a more realistic description of HPS, the acceptance based on the ECal was considered. New limits for the angles defining the fiducial region were obtained, using simulated electrons and positrons uniformly distributed in the energy region within $(0-2) \mathrm{GeV}$. The details of this acceptance study are given in the Appendix. Based on this acceptance study, new limits for the horizontal angles and out-of-plane angles depending 
on the particle momentum are found. However, due to the inefficiency of the Monte Carlo integration method to cover a very large phase space, the cross-section calculation in these full momentum dependent angular limits did not converge within the reasonable computing time, not allowing for meaningful results. For the present study, we thus used box-like limits, summarized in Tab. 5.3.4. These are larger and more realistic than the acceptance published in [125], while keeping the phase space to a reasonable size and avoiding numerical problems.

\begin{tabular}{|c||c|}
\hline BVMC & Kinematical Conditions \\
\hline \hline$E_{\text {beam }}$ & $1.056 \mathrm{GeV}$ \\
\hline$E_{\text {min }}^{e^{ \pm}}$ & $>0.15 \mathrm{GeV}$ \\
\hline$E_{\text {sum }}$ & $>0.5 \mathrm{GeV}$ \\
\hline$\left|\varphi_{\text {hor }}\right|$ & $<250[\mathrm{mrad}]$ \\
\hline$\left|\theta_{\text {out }}\right|$ & {$[-60,-15] \cup[15,60][\mathrm{mrad}]$} \\
\hline
\end{tabular}

Table 5.3.4.: Fiducial region used for the BVMC calculations.

The difference of acceptance region in $\theta_{\text {out }}$, obtained with the ECal geometry study (see the appendix sec. A.2.1), and the intervals in Tab. 5.3.3 for [125], affect the minimum mass accessible and other critical features. However, the $\theta_{\text {out }}$-coverage from the sec. A.2.1 is $\varphi_{\text {hor }}$-dependent, due to the cut-out region in the middle of the calorimeter. This dependence is not considered for the box-like limits in Tab. 5.3.4. The cut-out region is important because the forward processes are concentrated in that region. The limits considered for $\varphi_{\text {hor }}$ are widely different compared to the corresponding interval in Tab. 5.3.3. The simulation results show dependence with this variable for large masses. The electron energy, the positron energy as well as their sum are calculated with the simulations using the variables of the Set 1 from Tab. 5.3.2. The invariant mass distribution is obtained with simulations based on the variables of the Set 2. For the comparisons presented in the next section, the same acceptance limits are applied to the data simulated with MadGraph and with BVMC calculation.

\subsection{Comparison}

In this section, we compare the results from the different codes (MadGraph $4 / 5$ and BVMC) and examine the differences. The cross section is evaluated for the radiative trident ( $\mathrm{Rad}$ ) and Bethe-Heitler $(\mathrm{BH})$ processes, as well as for their interference $(\mathrm{Rad}+\mathrm{BH})$ and then, separately for the direct diagrams. The energy of the electron and positron, as well as the total energy distribution and the invariant mass distribution of the pair, are compared with different kinematical limits for the minimum lepton energy and the total energy. A series of plots are presented in Fig. 5.4.1, 
Fig. 5.4.2 and Fig. 5.4.3. The first column of each figure is the electron energy distribution, the second column is the positron energy, the third column is the total energy of the pair and the fourth column is the invariant mass distribution of the electron and positron pair. Each row corresponds to the calculation of a set of Feynman diagrams:

1. The first row is the full QED interaction diagrams, including the direct and exchange diagrams of radiative and $\mathrm{BH}$ processes (Fig. 5.3.4 and Fig. 5.3.5) with their interferences.

2. The second row corresponds to the radiative trident simulation with its direct and exchange terms, and their interferences (Fig. 5.3.4).

3. The third row is the radiative trident with only the direct diagrams (top two diagrams in the Fig. 5.3.4).

4. The fourth row corresponds to the Bethe-Heitler process including the direct and exchange diagrams of Fig. 5.3.5 and their interferences.

5. Finally, the fifth row is the cross-section of the BH process taking into account only the direct diagrams (top two diagrams of Fig. 5.3.5).

Note that MadGraph4 calculations are available only for rows 1, 3 and 5 .

Fig. 5.4.1 shows results with the following kinematical cuts: $E_{e^{+}, e^{-}}>0.15 \mathrm{GeV}$ and $E_{e+}+E_{e^{-}}>0.5 \mathrm{GeV}$. These are relatively loose cuts to test the calculation on a wide phase space. While the calculations agree for most of the kinematic variables and processes, there is a discrepancy between MG5, MG4 and the BVMC calculations at high $E_{\text {sum }}$ energies. Taking into account the electron and positron energy distributions, one can notice that the results hint to the discrepancy coming from the $\mathrm{BH}$ exchange terms.

Fig. 5.4.2 shows results with the following kinematical cuts: $E_{e^{+}, e^{-}}>0.15 \mathrm{GeV}$ and $E_{e+}+E_{e^{-}}>1.0 \mathrm{GeV}$. This set is generated to study the high $E_{\text {sum }}$ issue. We can confirm from the fourth and fifth rows that the difference comes from the $\mathrm{BH}$ exchange diagrams.

Fig. 5.4.3 shows results with the following kinematical cuts: $E_{e^{+}, e^{-}}>0.25 \mathrm{GeV}$ and $E_{e+}+E_{e^{-}}>0.8 \mathrm{GeV}$. This set is the closest to the one we will use for our actual analysis. The high $E_{\text {sum }}$ issue is still present, as well as the MadGraph4-MadGraph5 discrepancy. The BVMC calculation is closer to MadGraph5, in particular regarding the shape at low $E_{\text {sum }}$, indicating that the MadGraph5 event generator is likely better than MadGraph4.

The source of the discrepancy of these results can come from the difference in the way the two event generators handle the antisymmetrization. As another possible source of the divergences in the results, it was found for instance that the form factor used 
in the BVMC calculation and in the MG4/MG5 are different. It, however, seems unlikely that this small difference generates such strong discrepancy.

\subsection{Conclusion}

The dark photon production is swamped with $e^{+} e^{-}$pairs from interactions covering the HPS signal. The main backgrounds of the HPS signal, the prompt single vertex radiative process, wide angle bremsstrahlung and multiple Coulomb scatterings, were discussed in this chapter. The QED prompt background was simulated with the MadGraph4 and MadGraph5 event generator. The discrepancy between the outcome of these two generators and with the data motivated us to make an independent estimation of the same QED processes. In order to do so, an independent theoretical calculation, based on the work of Beranek and Vanderhaeghen [123, 125], is used. We applied a more accurate parametrization of the acceptance for these simulations. However, the implementation of the acceptance for a large $\varphi_{\text {hor }}$ range turned out to be problematic for the BVMC integration. Therefore, we used constant $\varphi_{\text {hor }}$ limits that adequately cover the ECal acceptance, making it manageable for the BVMC method. By attempting to recover the result of [125], it was found that the background was calculated for a positron beam instead of an electron beam. The comparison with the correct beam charge shows roughly a $20 \%$ difference.

The contribution from each piece of interaction was evaluated with BVMC and compared to MG4 and MG5. We found a discrepancy between MG5 and BVMC calculations for the $\mathrm{BH}$ process cross-section. The discrepancy shows in the total leptons energy, becoming more significant at larger $\left(E_{e^{+}}+E_{e^{-}}\right)$energies, highlighting the impact of exchange diagrams, where the scattered beam electron is part of the detected pair. We also confirmed that the results obtained with MG4 and MG5 differ significantly for the $\mathrm{Rad}+\mathrm{BH}$ processes over the full $E_{\text {sum }}$ range. 

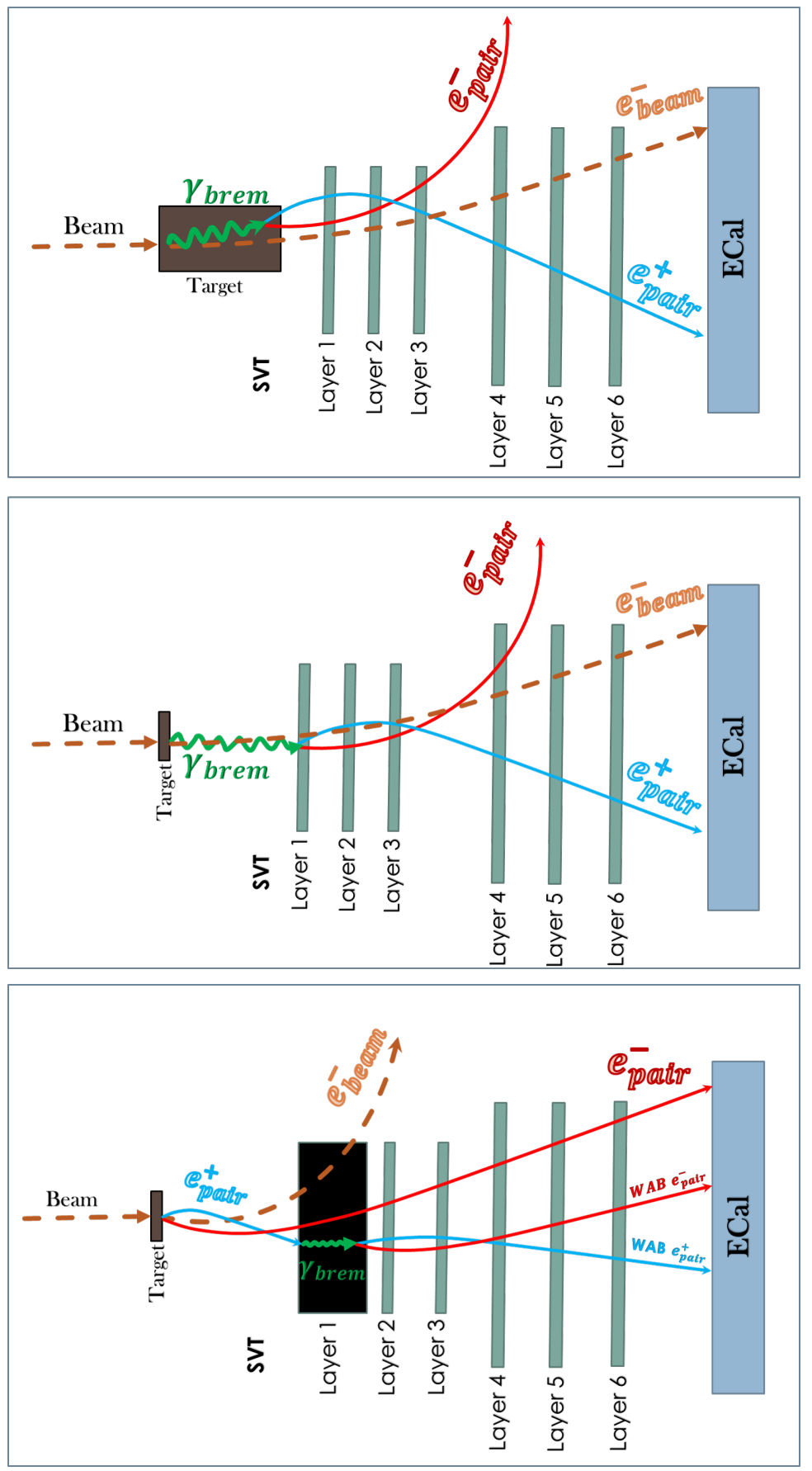

Figure 5.2.1.: Processes where a bremsstrahlung photon is converted into an electron-positron pair in the target or in the first layer of the SVT. The contribution from the bottom process is negligibly small. 


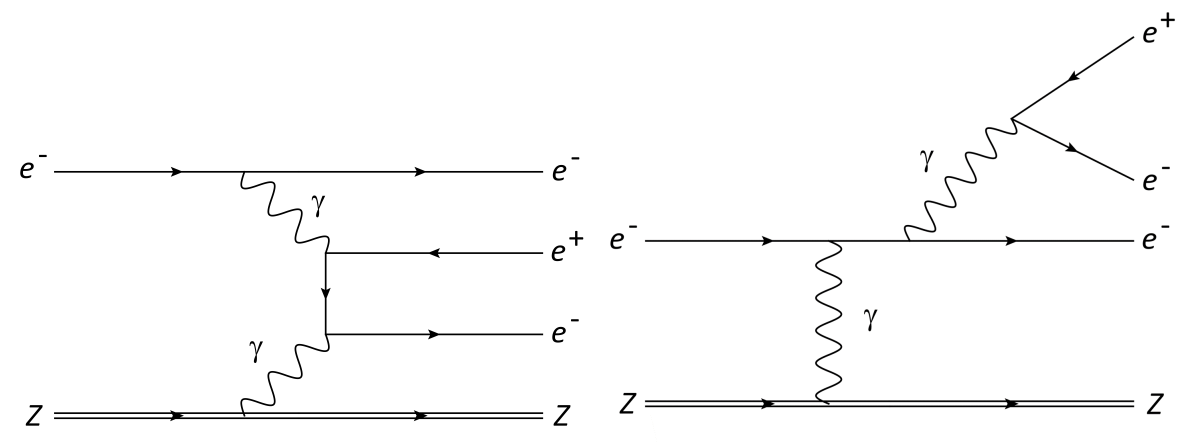

Figure 5.3.1.: Feynman diagrams of QED Bethe-Heitler trident (left) and radiative trident (right) processes.

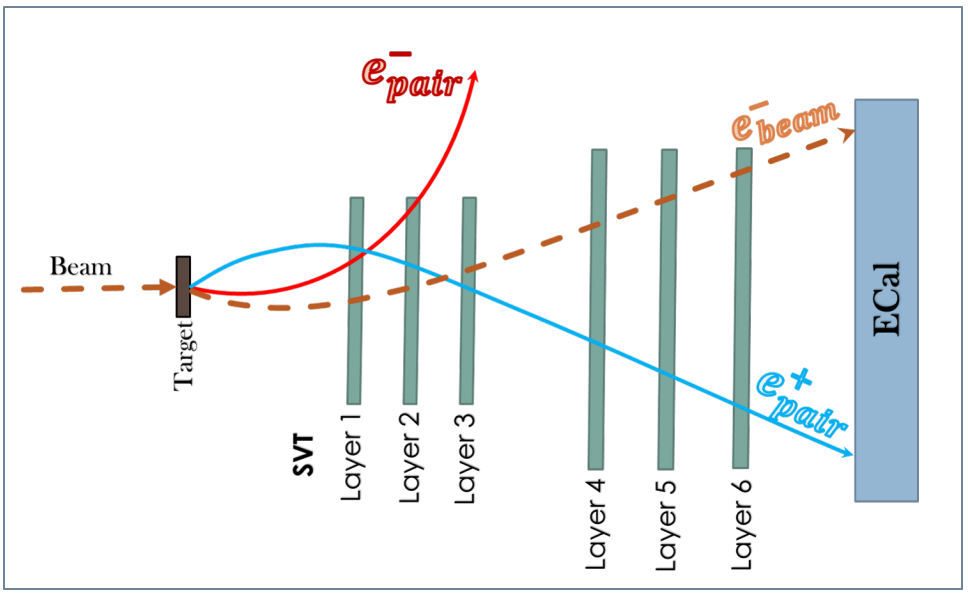

Figure 5.3.2.: Schematic of the electron-positron pair formation with the beam electron. 


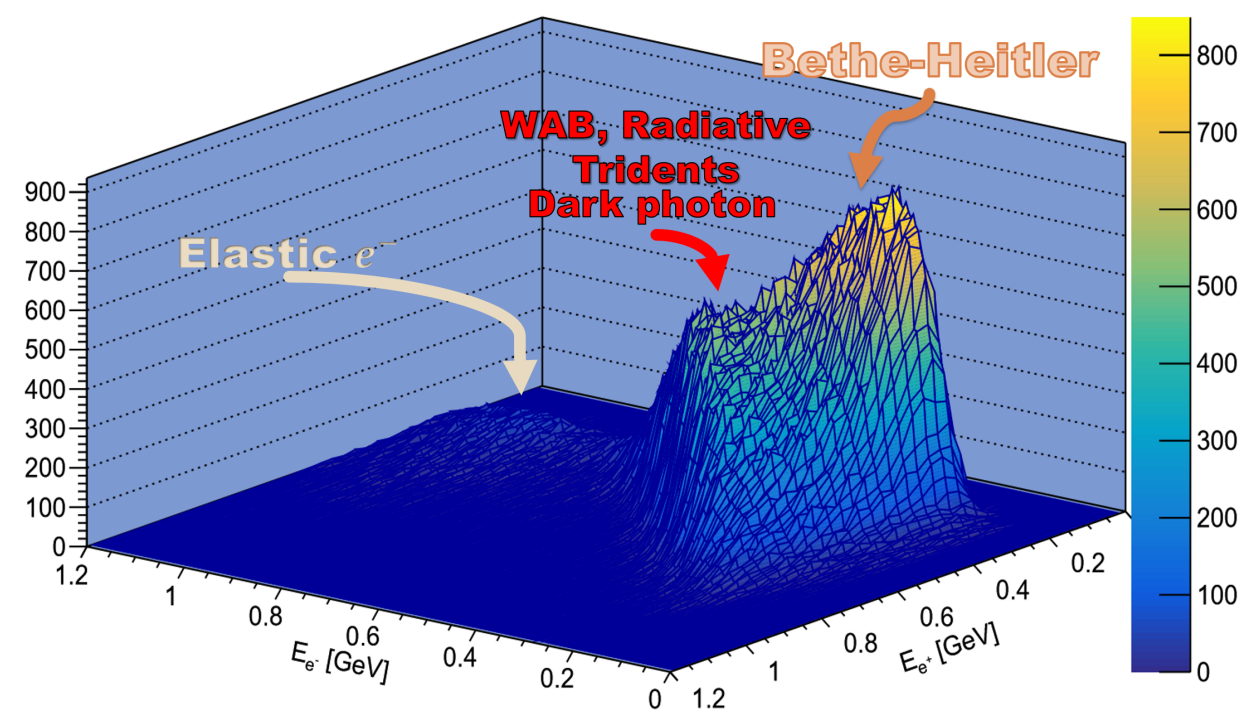

Figure 5.3.3.: The contribution of the different backgrounds as a function of the energy of the electron and the energy of the positron from the real data. Elastic electrons are coming from the high energy elastically scattered beam electrons.
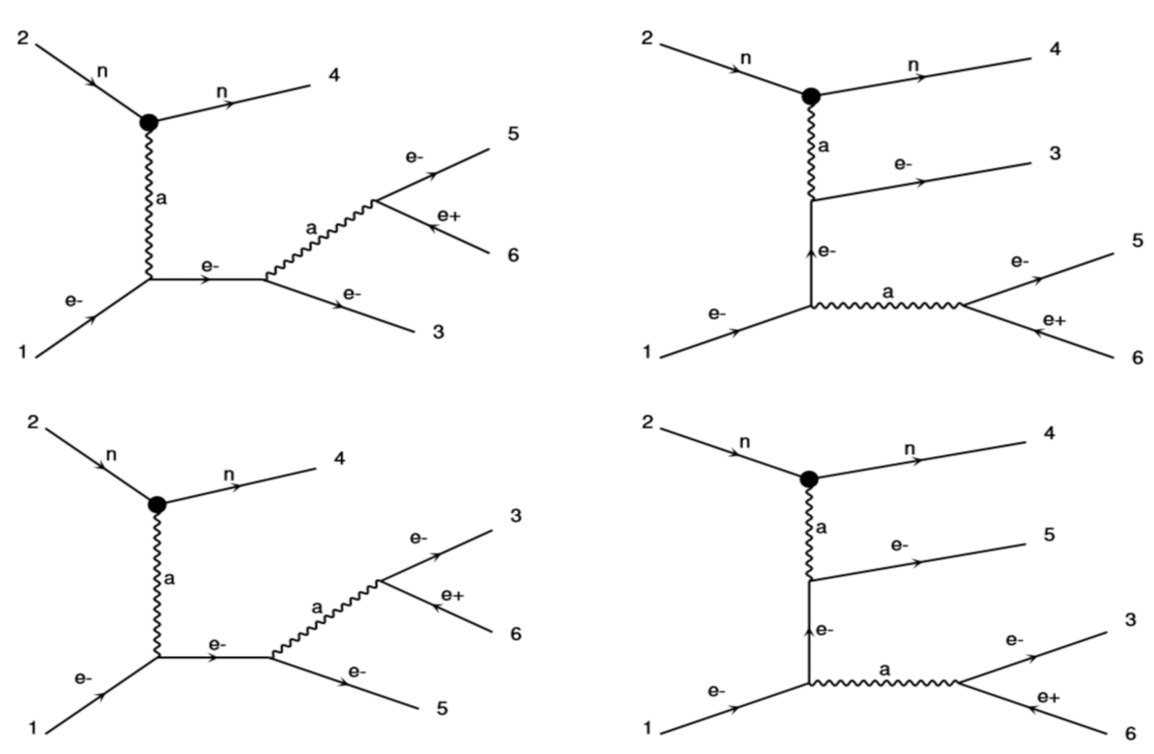

Figure 5.3.4.: Feynman diagrams of the radiative process. Photons are denoted as $a$ and the target nucleus as $n$. Bottom diagrams are those where the beam electron is exchanged with the pair electron. 

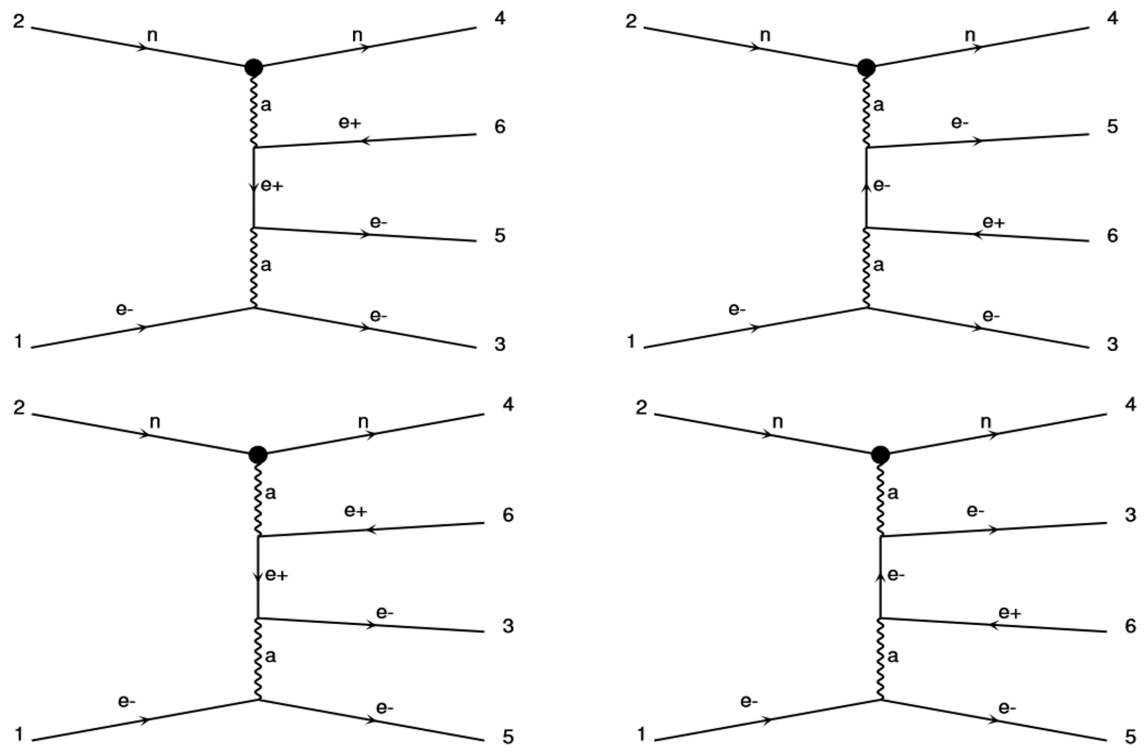

Figure 5.3.5.: Feynman diagrams of the Bethe-Heitler process. Radiated photons are denoted as $a$, the target nucleus as $n$. Bottom diagrams are those, where the beam electron is exchanged with the pair electron.

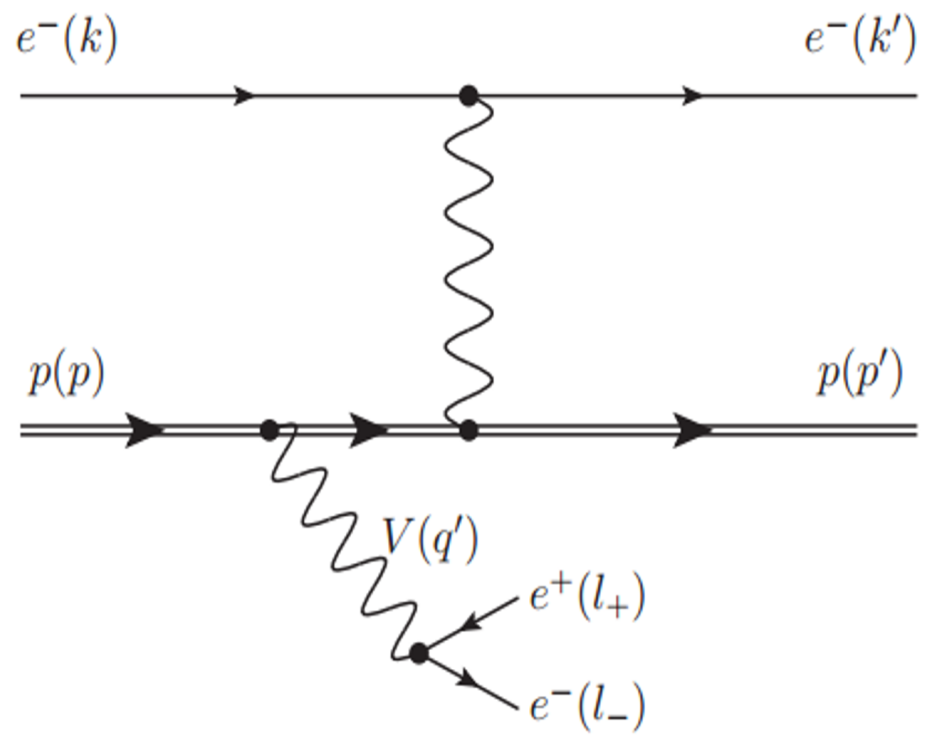

(VVCS)

Figure 5.3.6.: Feynman diagram of double virtual Compton scattering from [123]. 

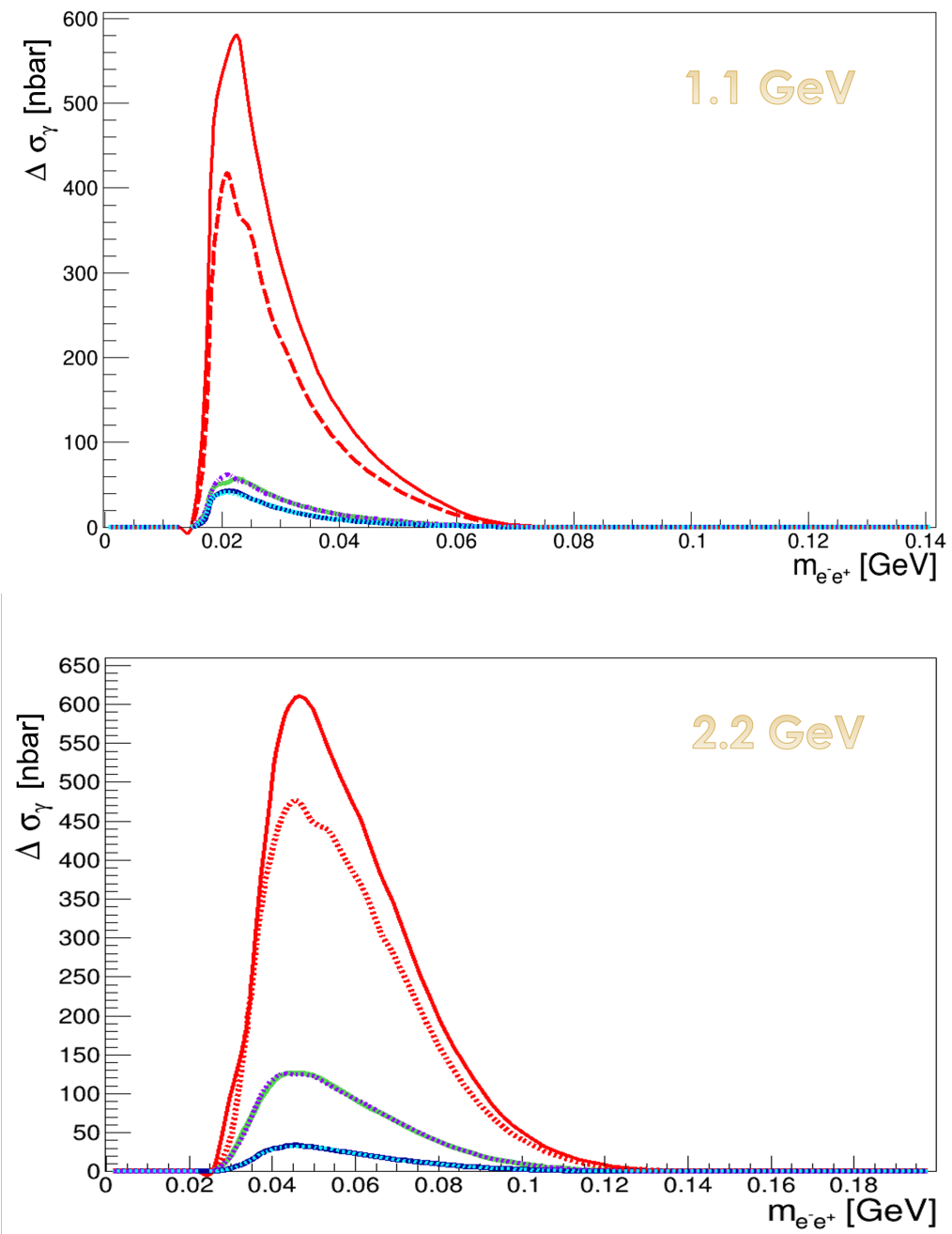

Figure 5.3.7.: The acceptance integrated total cross-section $\Delta \sigma$ as a function of the invariant mass of $e^{+} e^{-}$, calculated for $1.1 \mathrm{GeV}$ (top) beam energy and $2.2 \mathrm{GeV}$ (bottom) beam energy. The solid lines indicate calculations for an electron beam, dashed lines for a positron beam. The deep blue and light blue lines correspond to the direct radiative tridents, the green and purple lines are the cross-sections for the direct Bethe-Heitler diagrams and the red lines correspond to the radiative trident and Bethe-Heitler Feynman diagrams including the exchange diagrams. 

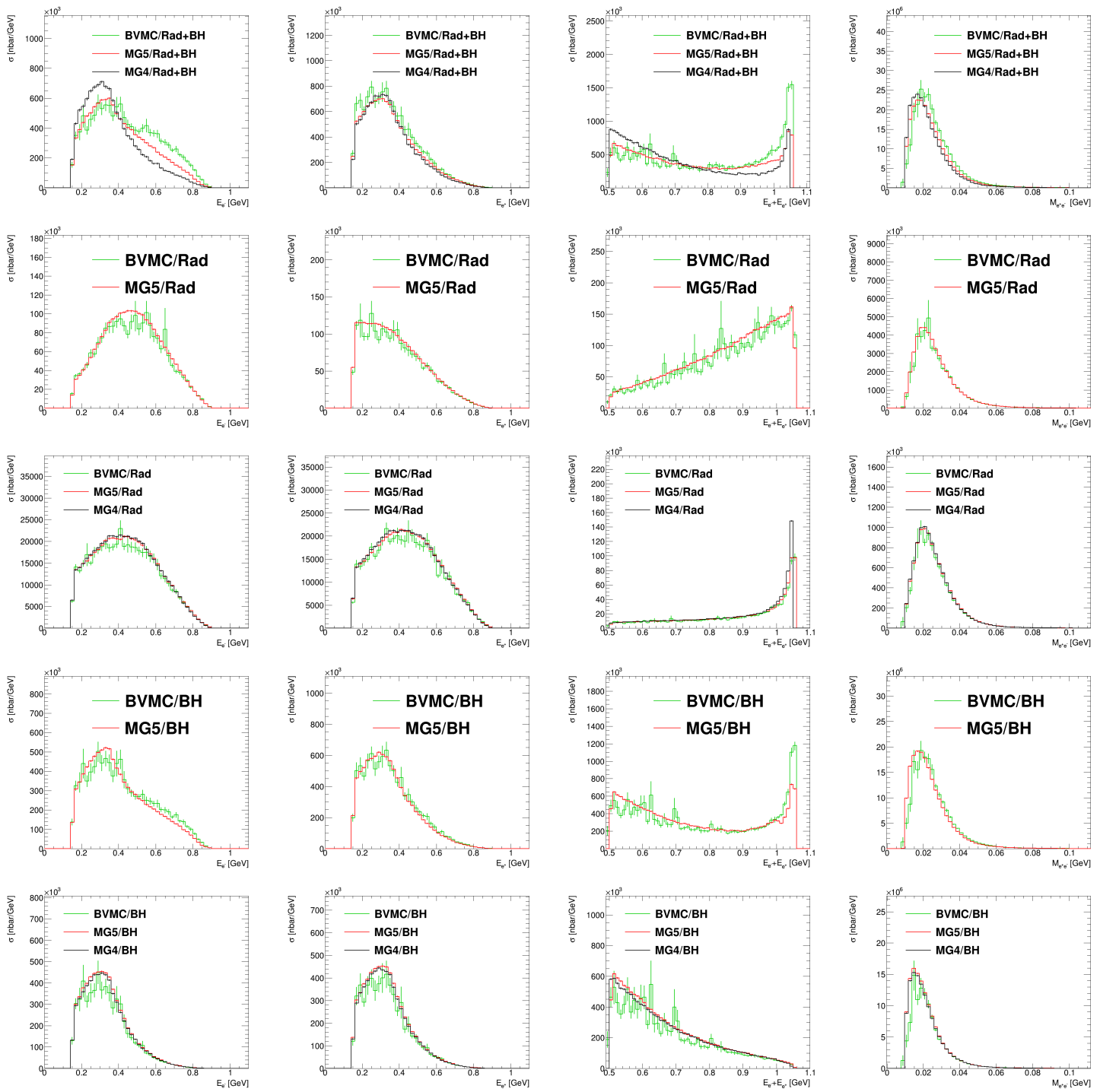

Figure 5.4.1.: The comparison of electron and positron energy distributions, their summed energy distribution and their invariant mass distribution for data samples generated by MadGraph4,5 (MG4 or MG5 indicated in the legend) and the BVMC method (BVMC in the legend), in the kinematic range: $E_{e^{+}, e^{-}}>0.15 \mathrm{GeV}$ and $E_{e+}+E_{e^{-}}>0.5 \mathrm{GeV}$. 

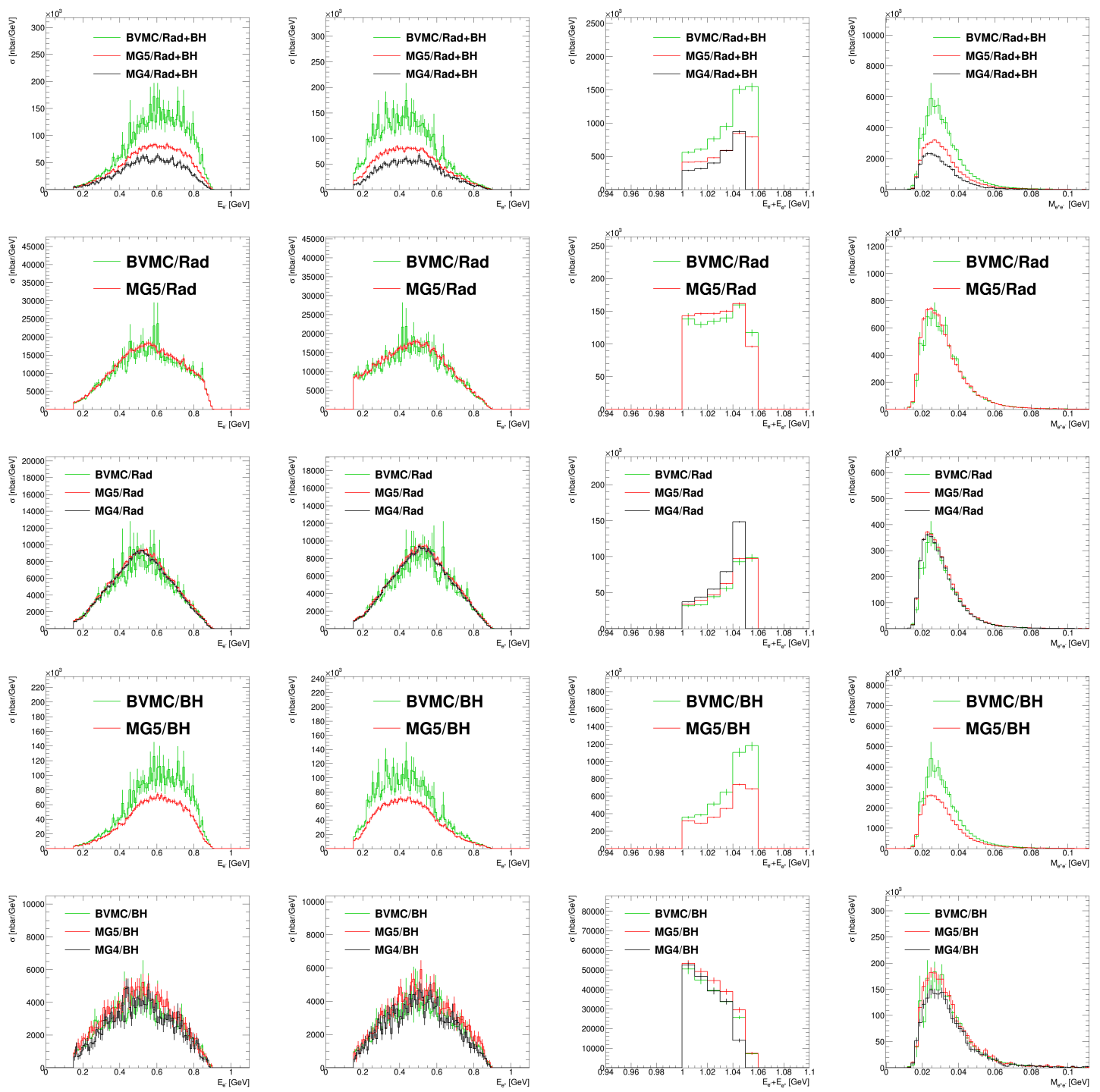

Figure 5.4.2.: The comparison of electron and positron energy distributions, their summed energy distribution and their invariant mass distribution for data samples generated by MadGraph4,5 (MG4 or MG5 indicated in the legend) and the BVMC method (BVMC in the legend), in the kinematic range: $E_{e^{+}, e^{-}}>0.15 \mathrm{GeV}$ and $E_{e+}+E_{e^{-}}>1.0 \mathrm{GeV}$. 

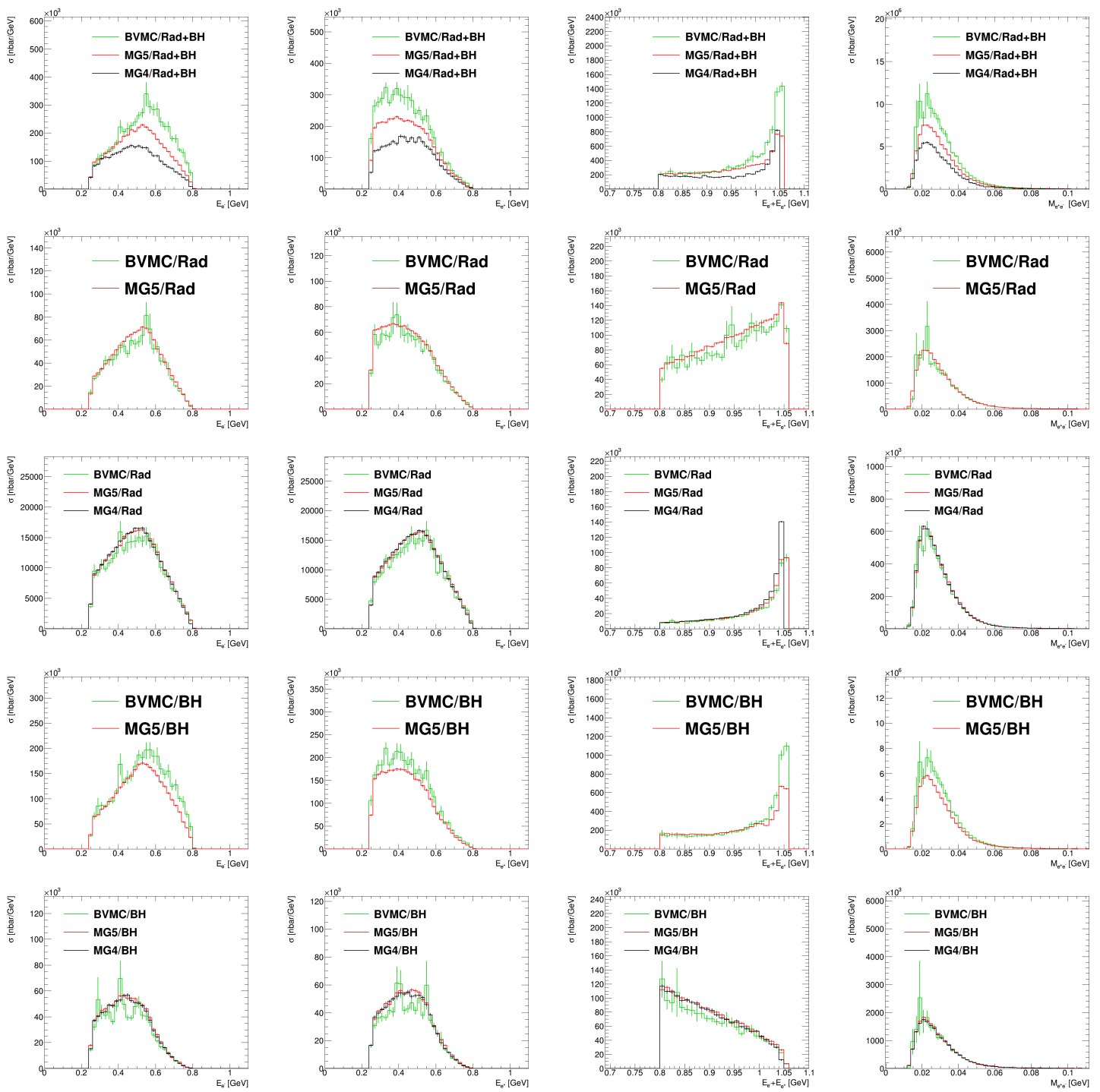

Figure 5.4.3.: The comparison of electron and positron energy distributions, their summed energy distribution and their invariant mass distribution for data samples generated by MadGraph4,5 (MG4 or MG5 indicated in the legend) and the BVMC method (BVMC in the legend), in the kinematic range: $E_{e^{+}, e^{-}}>0.25 \mathrm{GeV}$ and $E_{e+}+E_{e^{-}}>0.8 \mathrm{GeV}$. 


\section{Data Analysis: Moller electrons}

\subsection{The Moller Scattering}

Moller scattering, i.e. $e^{-} e^{-} \rightarrow e^{-} e^{-}$, has a kinematics similar to the HPS signal. This process is well known and can therefore be used to understand the resolution of the HPS detectors. In this chapter, the Moller scattering event selection and the invariant mass distribution of the Moller electrons are discussed.

Moller scattering is illustrated by the diagrams in Fig. 6.1.1. From the kinematic constraints, the energy $E$ and the scattering angle $\theta$ of the scattered electron are directly related:

$$
E(\theta)=\frac{E_{\text {beam }}}{1+\frac{2 E_{\text {beam }}}{m_{e}} \sin ^{2}\left(\frac{\theta}{2}\right)},
$$

where $m_{e}$ is the mass of the electron and $E_{\text {beam }}$ is the energy of the beam. Using eq.(6.1.1) and the energy conservation requirement allows one to select a rather clean set of events with Moller electron pairs. Solving equation (6.1.1) for the scattering
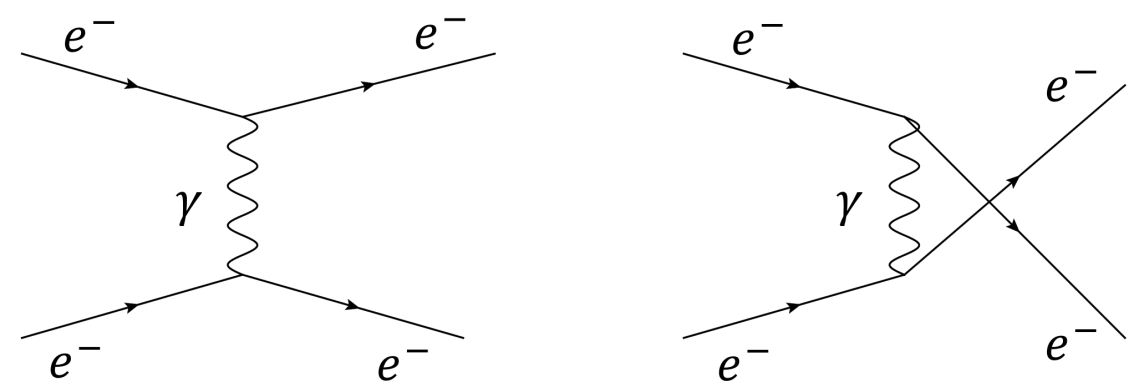

Figure 6.1.1.: Feynman diagrams for Moller scattering.

angle of one electron, $\theta_{e_{1}}$, as a function of the scattering angle of the second electron, $\theta_{e_{2}}$, we can express this constrain differently:

$$
\theta_{e_{1}}=2 \arcsin \left(\frac{m_{e}}{2 E_{\text {beam }} \sin \left(\frac{\theta_{e_{2}}}{2}\right)}\right)
$$

This dependence can be also expressed as follows:

$$
\sin \frac{\theta_{e_{1}}}{2} \sin \frac{\theta_{e_{2}}}{2}=\frac{m_{e}}{2 E_{\text {beam }}}
$$


These kinematic relations will help separate the Moller electron pairs from other processes.

The invariant mass of the Moller electron pair is defined as:

$$
m_{\text {inv }}^{2}=2 E_{\text {beam }} m_{e}+2 m_{e}^{2}
$$

\subsection{Moller Analysis}

\subsubsection{Data Sample}

The data samples used for the analysis include runs taken with the SVT positioned both at $0.5 \mathrm{~mm}$ (runs 5743, 5772, 5773, 5797) and at $1.5 \mathrm{~mm}$ (runs 5560, 5566, 5567, 5568, 5569, 5575, 5577, 5578, 5579) from the "pass6" reconstruction generation. Tab. 6.2.1 summarizes the data statistics for these runs. This chapter uses the plots from the $1.5 \mathrm{~mm}$ data sample, except at the end, where comparison of the invariant mas distributions of $1.5 \mathrm{~mm}$ and $0.5 \mathrm{~mm}$ data will be shown. The total number of Moller candidates in the $0.5 \mathrm{~mm}$ data is 18143 and 42500 Moller pairs in the $1.5 \mathrm{~mm}$ data sample, from which 1700 and 6569 respectively will survive after the selection cuts explained further down.

\begin{tabular}{|c|c|c|}
\hline Data sample & $\mathrm{N}_{\text {el. pairs }}$ - all & $\mathrm{N}_{\text {files }}$ \\
\hline \hline $0.5 \mathrm{~mm}$ setting & 18143 & 59 \\
\hline $1.5 \mathrm{~mm}$ setting & 42500 & 541 \\
\hline
\end{tabular}

Table 6.2.1.: Number of triggers with $e^{-} e^{-}$pairs in the data sample for the Moller analysis.

\subsubsection{Event Selection}

Reconstructed electron-electron pairs, found using the same vertexing algorithm as for electron-positron pairs, populate the "UnconstrainedMollerCandidates" and "TargetConstrainedCandidates" collections. In the latter case, the vertexing algorithm is forced to converge on the target, while in the former case this requirement is dropped as is the case for the dark photon displaced vertex search. The plots shown in the following to illustrate the event selection are all for the target constrained Moller pairs.

We start our analysis by requiring to have a "good" track and a matching cluster for the electrons. The minimum energy cut for a cluster in the EC is set to $100 \mathrm{MeV}$ and the seed hit timing of the cluster is taken within a window of 30-55 FADC samples. The track type, characterizing the strategy of the track finding is selected above 32 . 
This selects the tracks reconstructed with the GBL fitting algorithm (see sec. 4.3.2). The $\chi^{2}$ of the found track is also constrained with the reconstructed interaction vertex $\chi^{2}$. Eventually, the timing of each cluster is synchronized with a cut on the seed hit timing difference. Tab. 6.2.2 summarizes all the cuts described above.

The beam has a $-30.5 \mathrm{mrad}$ offset w.r.t. the $\mathrm{Z}$ axis in the laboratory frame. In order to account for this offset, the angles in all the calculations are rotated by this offset angle. The total energy of the pair, shown in Fig. 6.2.1, should be equal to

\begin{tabular}{|c|c|}
\hline Selection for each particle & Value \\
\hline \hline Cluster Energy & $>100 \mathrm{MeV}$ \\
\hline Cluster Seed Hit Timing & $30-55$ FADC samples \\
\hline Track Type & $>32$ \\
\hline Track $\chi^{2}$ & $<20$ \\
\hline Vertex $\chi^{2}$ & $<10$ \\
\hline Cluster-cluster timing & $<3 \mathrm{~ns}$ \\
\hline
\end{tabular}

Table 6.2.2.: Track and cluster selection criteria for our Moller analysis.

the beam energy by simple energy conservation and serves for our first kinematical cut. The peak corresponding to the beam energy is modeled by a Gaussian and a third order polynomial. From the Gaussian's mean parameter, the peak's center was found at $1.044 \mathrm{GeV}$, the nominal beam energy being $1.056 \mathrm{GeV}$. The difference is about $10 \mathrm{MeV}$. It is small and within calibration precision. The total energy peak position for unconstrained and target constrained electrons are slightly different: $1.048 \pm 0.0001 \mathrm{GeV}$ and $1.044 \pm 0.001 \mathrm{GeV}$ respectively. In the following, we use the nominal energy for $E_{\text {beam }}=1.056 \mathrm{GeV}$.

The energy distribution of one electron versus the other one is displayed in Fig. 6.2.2. These are Moller candidate events. We see the $3 \sigma$ cut calculated based on the Gaussian fit of Fig. 6.2.2 (plotted as red solid lines). The events where $E_{e_{1}}=E_{\text {beam }}$ and $E_{e_{2}}=E_{\text {beam }}$ are accidentals from the elastically scattered full energy electron and a soft electron pair. To filter out these accidentals, each electron is required to have more than $25 \%$ and less than $85 \%$ of the beam's energy.

For further refinement of our sample, we use the kinematical constraints for the scattering angles from eq.(6.1.2). The scattering angle of one electron versus the scattering angle of the other one are plotted in Fig. 6.2.3, together with the expectations from eq.(6.1.2). We filter out the electron pairs that are not from Moller scattering by requiring the scatter angle to be less than $60 \mathrm{mrad}$ and the sum of the scatter angles to be limited by $90 \mathrm{mrad}$. Events surviving after this selection are plotted in Fig. 6.2.4.

Another Moller selection cut can be derived from eq.(6.1.3), where the product of the sines of the scattering angles is constrained by the beam energy and the mass of the electron: $\sin \frac{\theta_{e_{1}}}{2} \sin \frac{\theta_{e_{2}}}{2}=2.414773 \times 10^{-4}$ for a beam energy $E_{\text {beam }}=1.056 \mathrm{GeV}$. 


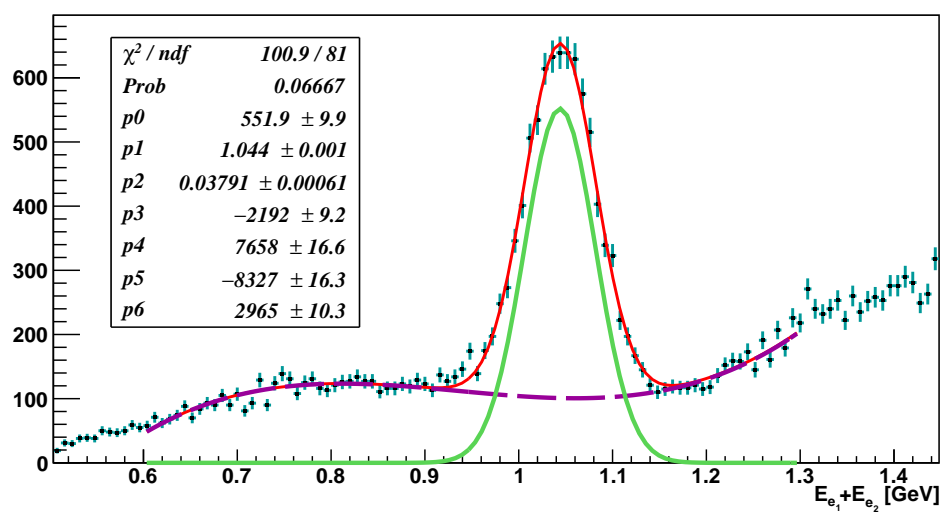

Figure 6.2.1.: The distribution of the energy sum of the electron pairs, fitted with a composite function of a Gaussian (parameters p1 and p2 are respectively the mean and the width) plus a third order polynomial.

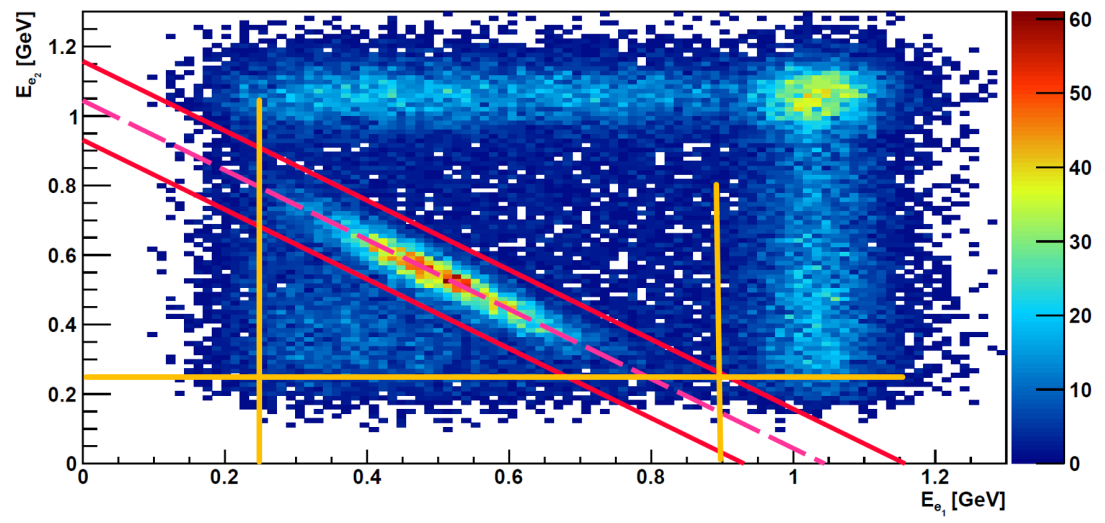

Figure 6.2.2.: The distribution of the energy of one of the electrons versus the energy of the second one. Red solid lines represent the $E_{\text {beam }} \pm 3 \sigma$ cut interval. The orange lines are the cuts for the electron energy in the range $25 \% E_{\text {beam }}<\mathrm{E}_{e}<85 \% E_{\text {beam }}$. 


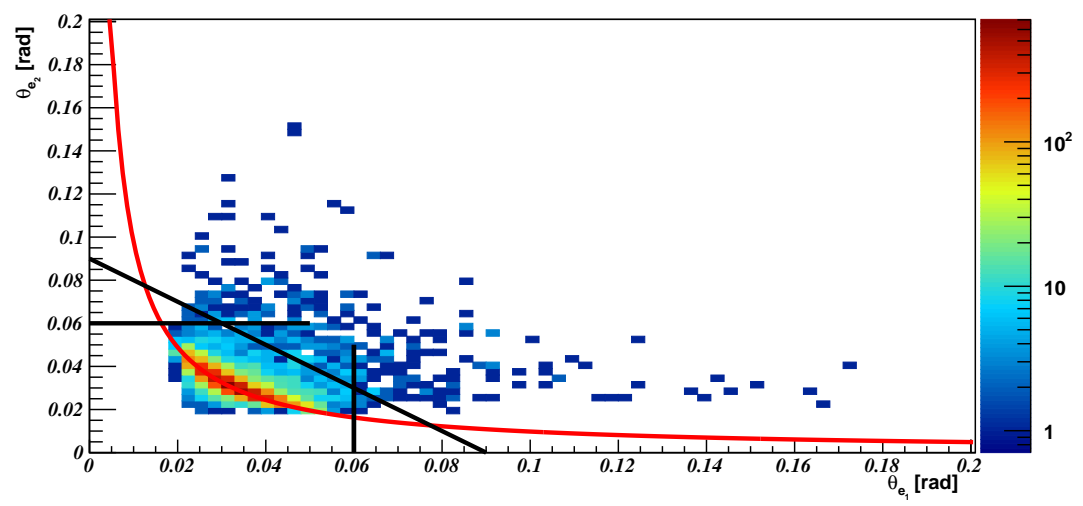

Figure 6.2.3.: The scattering angle of one electron as a function of the other one. The red solid line corresponds to eq.(6.1.2) and the black lines are the cuts applied at $60 \mathrm{mrad}$ for each particle and $90 \mathrm{mrad}$ for the sum.

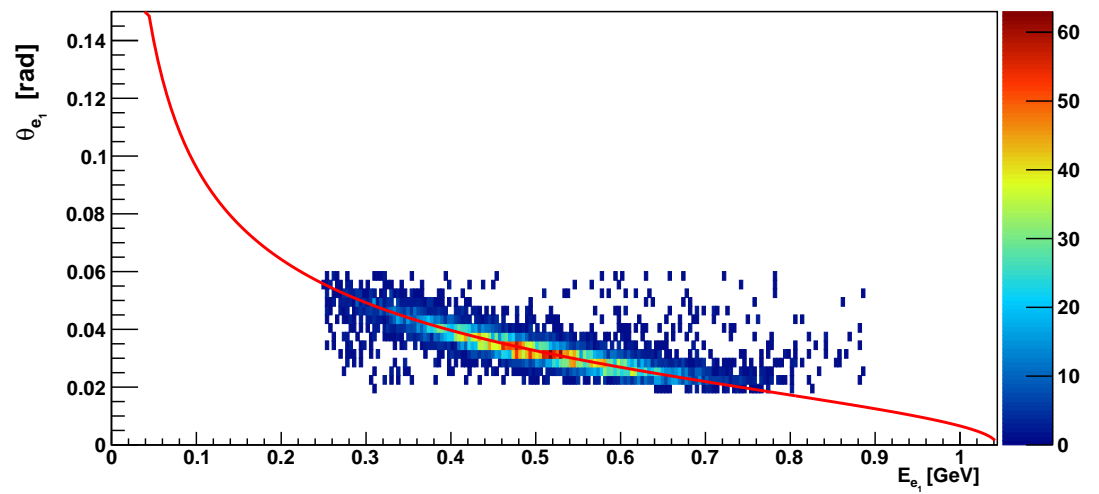

Figure 6.2.4.: The distribution of the electron scattering angle versus its energy, where the red solid lines represent the theoretical estimate of $e^{-} e^{-} \rightarrow e^{-} e^{-}$elastic scattering.

The product of the sines of the electron scattered angles is plotted and fitted with a sum of a Gaussian, modeling the peak, and a second order polynomial for the background, in Fig. 6.2.5. Ideally, the peak position should be at $\mathrm{x}=2.414773 \times 10^{-4}$. However, due to measurement imperfections, the value is found to be shifted to: $\mathrm{x}=2.519 \times 10^{-4} \pm 0.003 \times 10^{-4}$. This mismatch shows that there are still some alignment issues with the SVT or possibly target position issues. A selection cut is made up of the $3 \sigma$ region around this latter value. This concludes all the selection criteria used for the Moller scattering selection. 


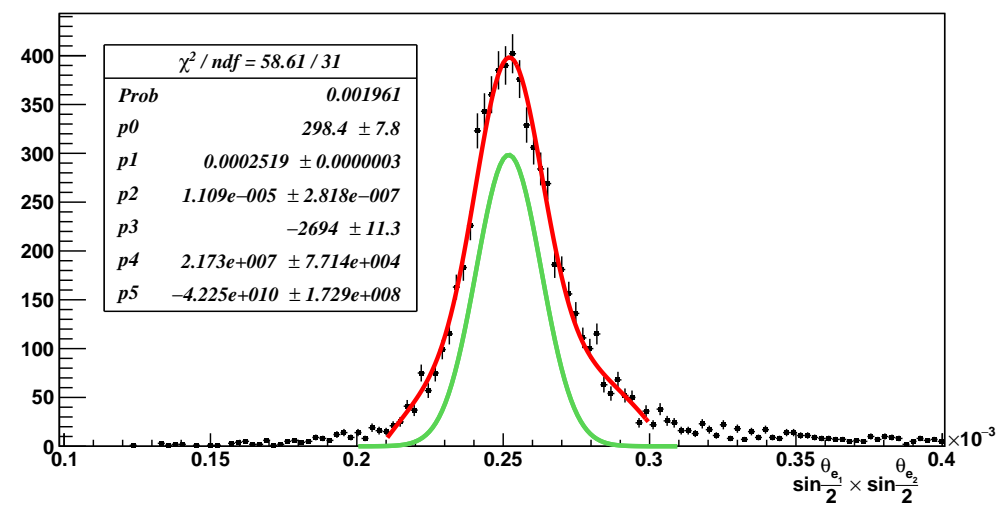

Figure 6.2.5.: The distribution of the product of the sines of $\frac{1}{2} \theta_{1}$ and $\frac{1}{2} \theta_{2}$, fitted with a composite function (red line) containing a Gaussian (green line) to model the peak with parameters $\mathrm{p} 0$ as the amplitude, p1 as the mean and p2 as the width of the Gaussian and a second order polynomial to model the background (p3, p4, p5).

\subsubsection{Results}

Since Moller scattering is elastic, the electrons scatter in opposite directions in the $\mathrm{XY}$ plane, meaning the azimuthal angles of the electrons are opposite with a $180^{\circ}$ angle, with respect to each other. This is illustrated in the distribution in Fig. 6.2.6. We find that the difference of the azimuthal angles is, as expected, centered around 0 , with our fit giving a Gaussian mean of $-0.01461 \mathrm{rad}$ and a width of $0.09051 \mathrm{rad}$. The low background level can be viewed as an estimation of the quality of the Moller electron pair selection, while the width is a measurement of the resolution of the azimuthal angle of the HPS detector.

From eq.(6.1.4), the theoretical value of the invariant mass of the Moller electron pair $m_{i n v}=0.0325 \mathrm{GeV}$ for $E_{\text {beam }}=1.056 \mathrm{GeV}$. The invariant mass distribution of Moller electrons can be used to estimate the mass resolution of the detector at $m_{i n v}$. To extract this information, the invariant mass spectrum is considered and fitted by a Gaussian+polynomial function. A similar analysis is carried out for the data sample using the $0.5 \mathrm{~mm}$ SVT position setting. In Fig. 6.2.7, the invariant mass of the electron pairs with unconstrained vertex from the $0.5 \mathrm{~mm}$ setting is shown, fitted by a Crystal Ball function [126] to take into account some asymmetry in the distribution. Here the $\mathrm{p} 2=0.03311 \mathrm{GeV}$ and $\mathrm{p} 3=0.00253 \mathrm{GeV}$ parameters correspond to the peak position and width respectively. The invariant mass distribution for the $1.5 \mathrm{~mm}$ data sample is shown in Fig. 6.2.8, where the results from unconstrained vertex are on top and the results from target constrained vertex are at the bottom. In this case, the peak is better modeled with a Gaussian (p0, p1, p2 parameters) and a second order polynomial (p3, p4, p5 parameters). The mean of the Gaussian for target constrained vertex pairs is $\mathrm{p} 1=0.03313 \mathrm{GeV}$ and a width is $\mathrm{p} 2=0.00154 \mathrm{GeV}$. 


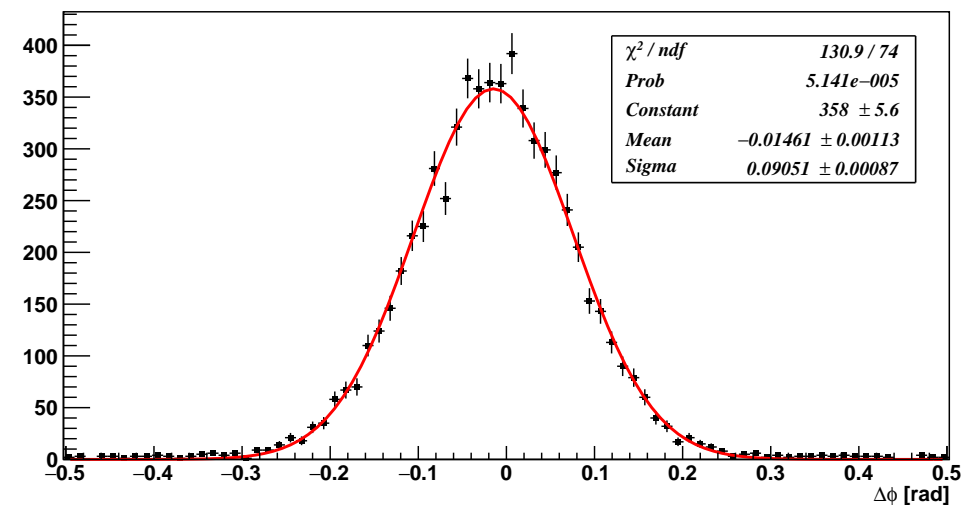

Figure 6.2.6.: The difference between the azimuthal scattering angle of one electron and the second. The red line is the Gaussian fit to the peak.

The unconstrained vertex pairs results show a mean of $\mathrm{p} 1=0.03331 \mathrm{GeV}$ and a width of $\mathrm{p} 2=0.00216 \mathrm{GeV}$. Therefore, the mass resolution is respectively $2.5 \mathrm{MeV}$ and $2.2 \mathrm{MeV}$ for the $0.5 \mathrm{~mm}$ and for the $1.5 \mathrm{~mm}$ data samples with unconstrained vertex pairs and $1.5 \mathrm{MeV}$ for the $1.5 \mathrm{~mm}$ data sample with vertex of electron pair constrained at the target. For these studies, the mass is at $33.1 \mathrm{MeV}$ for the $1.5 \mathrm{~mm}$ data sample with target constrained vertex pairs and for the $0.5 \mathrm{~mm}$ data sample with unconstrained vertex pairs, while it is at $33.3 \mathrm{MeV}$ for the $1.5 \mathrm{~mm}$ data sample with unconstrained vertex pairs. The results from target-constrained pairs therefore show a finer mass resolution than the result for the unconstrained set. In the following analysis, the target-constrained sets are used.

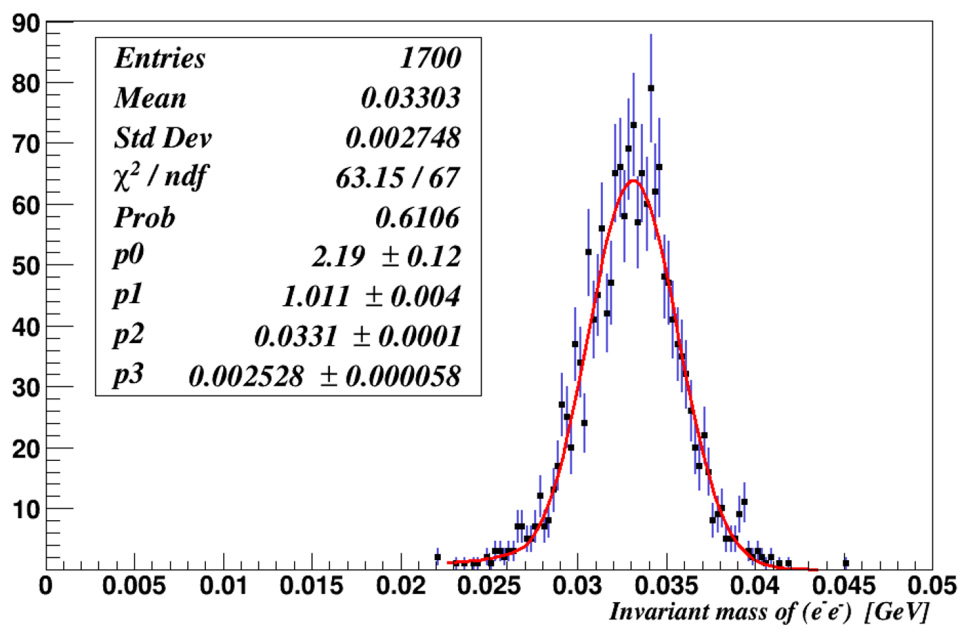

Figure 6.2.7.: Distribution of the invariant mass of the electron pairs from the data sample with a $0.5 \mathrm{~mm}$ SVT setting and with unconstrained interaction vertex. The red line is the fit of the peak with a Crystal Ball function [126]. 


\subsection{Conclusion}

Moller scattering has been studied with the $1.5 \mathrm{~mm}$ and $0.5 \mathrm{~mm}$ data samples. Tab. 6.3.1 summarizes the invariant mass distribution for each data set depending on the vertexing strategy. Both data sets show better mass resolution with the target constrained set. The theoretical invariant mass value calculated with eq.(6.1.4) agrees with the experimental results within a few percent.

\begin{tabular}{|c|c|c|c|c|}
\hline \multirow{2}{*}{ Data sample } & \multicolumn{2}{|c|}{ Unconstrained } & \multicolumn{2}{c|}{ Target Constrained } \\
\cline { 2 - 5 } & mean & width & mean & width \\
\hline \hline $0.5 \mathrm{~mm}$ setting & 0.0331 & 0.0025 & 0.0332 & 0.0014 \\
\hline $1.5 \mathrm{~mm}$ setting & 0.0333 & 0.0022 & 0.0331 & 0.0015 \\
\hline
\end{tabular}

Table 6.3.1.: The mean and the sigma of the invariant mass distribution for the

$1.5 \mathrm{~mm}$ and $0.5 \mathrm{~mm}$ data with unconstrained and target constrained vertex. The values quoted for the target constrained set in the $0.5 \mathrm{~mm}$ data is taken from [127]. 

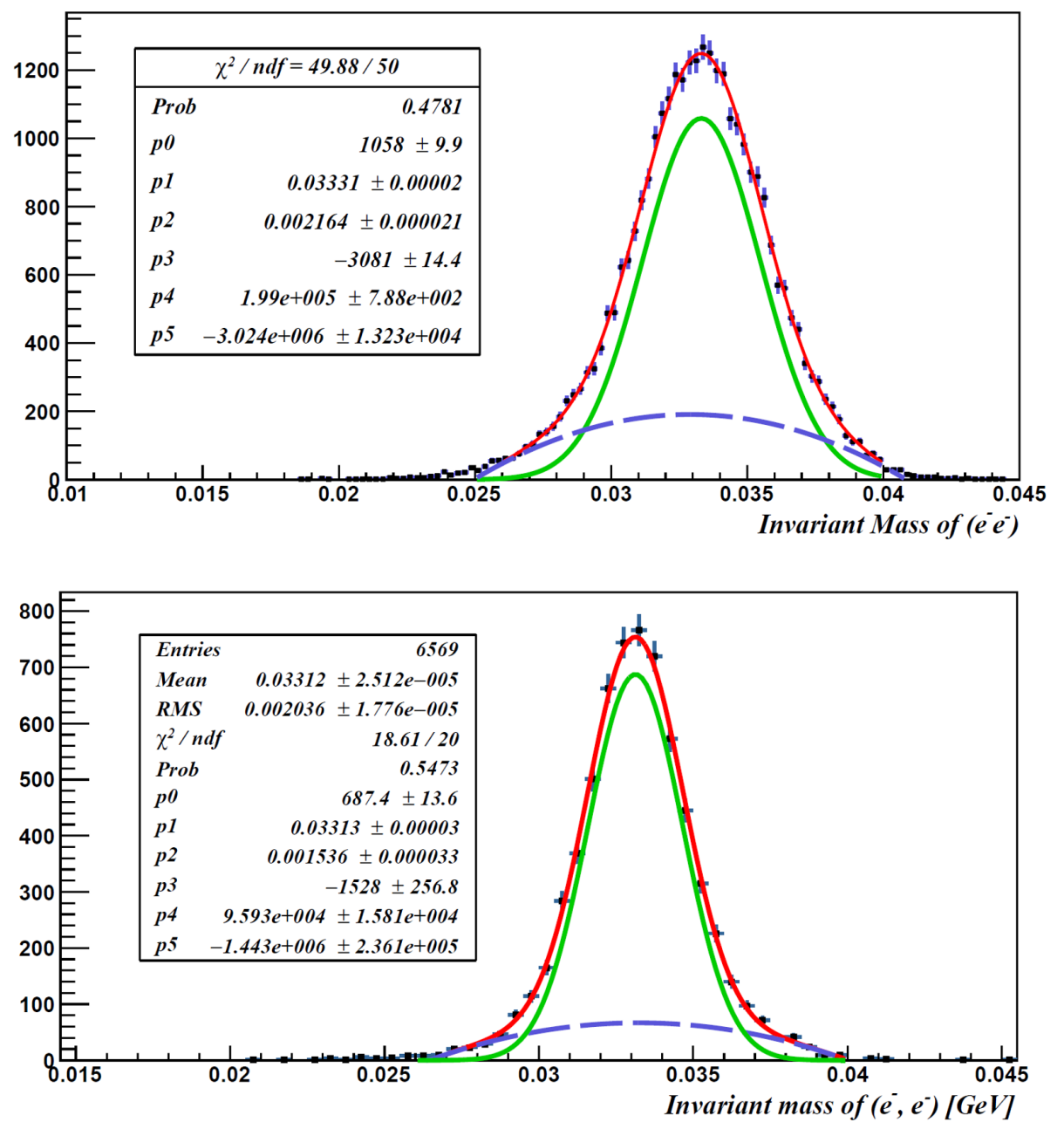

Figure 6.2.8.: Distribution of the invariant mass of the electron pairs from the data sample with the SVT at $1.5 \mathrm{~mm}$ with unconstrained vertex in the top and with target constrained vertex in the bottom. The red line is a fit using a composite function, consisting of a Gaussian (green solid line) and a second order polynomial (purple dashed line). 



\section{Data Analysis: Dark Photon Search}

\subsection{Introduction}

The focus of this analysis is to look for a dark photon decaying into an electronpositron pair, which can be observed as a small bump in the invariant mass spectrum on top of a continuous QED background. We present here our search for this signature in the 2015 HPS data with the SVT at $1.5 \mathrm{~mm}$. A separate analysis for the dark photon displaced vertex search is pursued by other members of the collaboration.

In the first stage of the analysis, we select events that are in favorable kinematics for dark photon production and plot the corresponding invariant mass spectrum. In the second stage, the invariant mass distribution is scanned for a resonance search. In case of a negative result, we use this analysis to determine the exclusion regions that can be drawn from our search.

\subsection{Dark photon production}

The Feynman diagrams for dark photon production followed by electron-positron pair formation are shown in Fig. 7.2.1. These illustrate the signal that the HPS experiment is looking for. The properties of this process are discussed in more details in sec. 1.4.5. The dark photon production is a rather rare event suppressed in comparison with SM virtual photon production. However, a number of factors favoring this process can be exploited to accumulate enough statistical power for an observation. Here, we review the main criteria used in the analysis to isolate the relevant signal from the data:

1. The dark photon production cross section is largest when it carries almost all the incident electron energy.

2. The dark photon is mainly produced at small angles with respect to the incident beam. This promotes a forward decay to an electron-positron pair registered in opposite halves of the detector.

3. The $e^{+}$and $e^{-}$are likely to have balanced energies.

The reflection of these requirements is already implemented in the trigger logic explained in sec. 3.6.1. We describe below the refinements on this sample. 


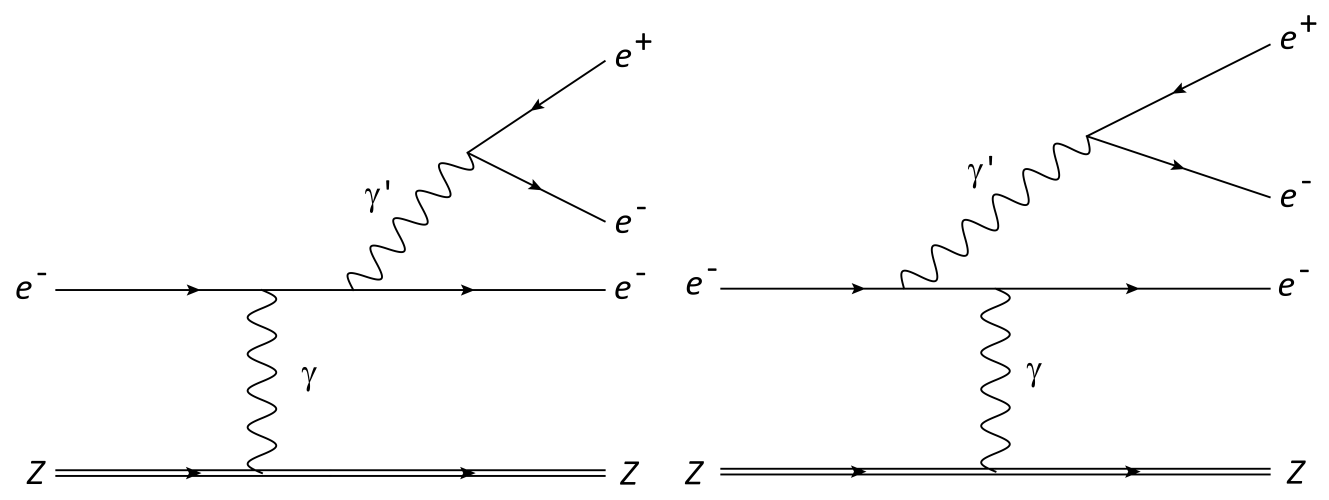

Figure 7.2.1.: The Feynman diagrams of dark-strahlung searched in the HPS Experiment.

\subsubsection{Event Selection}

The vertexing algorithm generates different pairs of reconstructed electron and positron particles. A comparison of the event selection based on the target constrained and the unconstrained sets did not show any significant difference in their kinematics other than the vertex position. Since the latter is not of any particular interest for a bump hunt search and since the mass resolution was found to be better with the target constrained data set, we use pair candidates from this latter set.

Despite the trigger requirement of two particles being detected in opposite halves of the ECal, the vertexing algorithm often forms pairs from clusters in the same module of the calorimeter. In the offline analysis, this condition is checked and irrelevant pairs are rejected. Next, to make sure all reconstructed particles have a good signal in the tracker and in the calorimeter, basic selection criteria for tracks and clusters are implemented. They are summarized in Tab. 7.2.1.

\begin{tabular}{|c|c|}
\hline Selection for each particle & Value \\
\hline \hline Cluster Energy & $>150 \mathrm{MeV}$ \\
\hline Cluster Seed Hit Timing & $30-55$ FADC samples \\
\hline Track Type & $>32$ \\
\hline Track $\chi^{2}$ & $<20$ \\
\hline
\end{tabular}

Table 7.2.1.: Track and cluster selection criteria. Track type here stands for the tracking fit algorithm. Selected tracks with type $>32$ correspond to tracks found via the final GBL fit (see sec. 4.3.2 for details).

In addition, to reduce accidentals, the timing of the clusters are selected to match. The seed hit timing (most energetic hit in the cluster) difference between the electron and the positron is shown in Fig. 7.2.2. The peak is fitted with a Gaussian and a second order polynomial, then the events are selected using a mean $\pm 3 \sigma$ cut. 


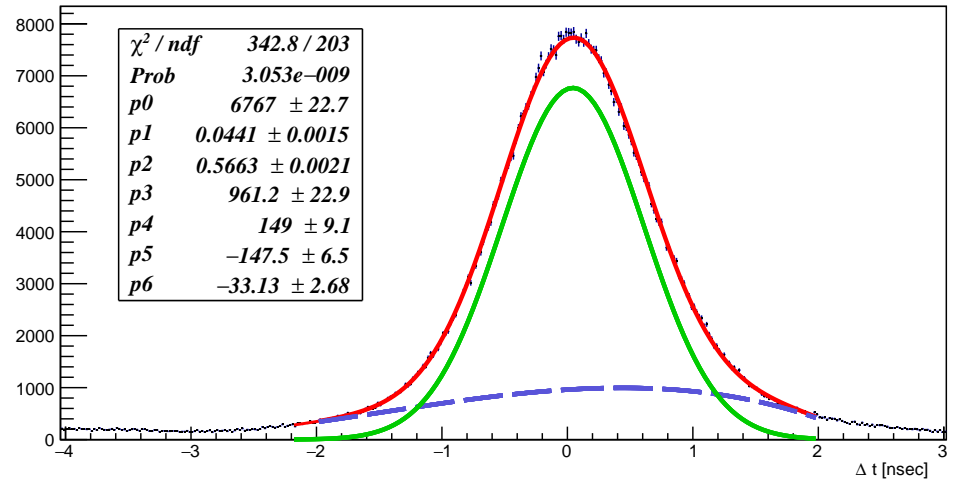

Figure 7.2.2.: The cluster timing difference between the electron and the positron, fitted with a composite function of a Gaussian (green line, p0, p1-mean, p2-width) and a third order polynomial (blue dashed line).

In Fig. 7.2.3 and in Fig. 7.2.4, we can observe that the energy distribution of the electrons has a small bump around the beam energy, $1.056 \mathrm{GeV}$. These electrons correspond to low-angle scattered full beam energy electrons (FEE) in coincidence with low energy accidental positrons. In Fig. 7.2.3, the red line is a fit to the bump and the dashed black line indicates the cut at the local minimum to exclude these FEE events. The cut is set at a value of $0.85 \mathrm{GeV}$, which is equivalent to $\sim 2 \sigma$ from the Gaussian mean.

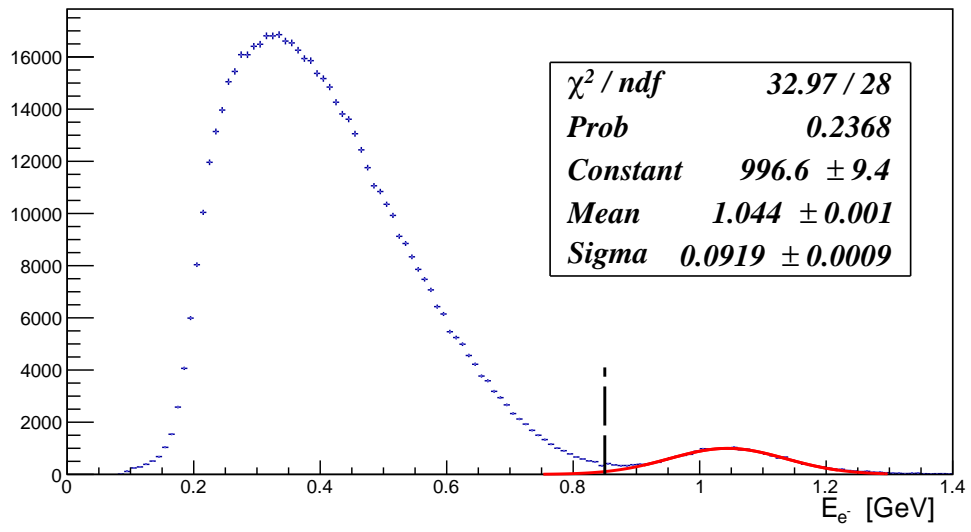

Figure 7.2.3.: The electron energy distribution. The red line corresponds to the Gaussian fit to the FEE bump. The black dashed line represents the cut implemented to exclude the pairs with a FEE electron.

Dark photon production is accompanied by an intense QED background from ordinary bremsstrahlung and radiative processes. This process is discussed in detail in chapter 5. The cross section of the $\mathrm{BH}$ process are largest at low lepton pair energies, such that large part of it can be cut out. In the positron energy versus electron 
energy distribution, displayed in Fig. 7.2.4, the BH radiatives are concentrated at small energies. There is a tail from $\mathrm{BH}$ events that contaminates the region of energy sum for the radiative tridents and dark photons. In order to filter out most of the contribution from this process, only pairs with a total energy larger than $0.8 \mathrm{GeV}$ are accepted.

At this point, there can still be multiple candidates per event, often with a cluster or a track common to two or more $e^{-} e^{+}$pairs. We select only one candidate per event, based on the reconstructed vertex $\chi^{2}$ parameter, requiring $\chi_{\min }^{2}<20$. In Fig. 7.2.5, the reconstructed vertex distribution is plotted for pairs before and after the cuts described up to this point. We can see that we have now a much cleaner data sample.

The out-of-plane angle and the horizontal angle are plotted in Fig. 7.2.6. This gives a way to estimate the fiducial region based on data. Comparing with the acceptance obtained from the simulations in Tab. 5.3.4, it justifies the acceptance region selected in the background simulations. The plot shows that $\theta_{\text {out }}$ varies in a similar range than considered in the proposal [63] (from $\pm 15 \mathrm{mrad}$ to $\pm 60 \mathrm{mrad}$ ), which we also use for our calculation in chapter 5 . However, rates drop significantly before reaching these acceptance limits, therefore suggesting that the actual acceptance is smaller than expected from the proposal. This observation is consistent with our study of the ECal acceptance, discussed in the Appendix. Such a tight fiducial region affects the reach of the experiment, reducing the expected coverage in $\epsilon$ coupling vs dark photon mass phase space.

The position of the particles at the ECal can be calculated from the trajectories measured in the tracker and from the reconstructed position from the ECal cluster information. The difference in these measurements in $\mathrm{X}$ and $\mathrm{Y}$ coordinates are plotted in Fig. 7.2.7 and in Fig. 7.2.8, respectively, for electrons (left) and positrons (right). There is no significant shift in the $\mathrm{Y}$ direction, but the $\mathrm{X}$-distributions in Fig. 7.2.7 deviate from zero for both electrons and positrons. The cause of this shift can be a misalignment of the ECal position relative to SVT layers, another source of the shift can be the asymmetric displacement of the crystals from their nominal position illustrated in Fig. 4.2.2. In this analysis, the data sample is from the reconstruction generation "pass6", where the ECal geometry correction discussed in sec. 4.2.1 is not applied yet, which could potentially explain the problem. The next generation data processing will show if this alignment issue is resolved. In any case, this problem is not vital for the bump hunt analysis, which mostly depends on the mass resolution and particle momentum measured in the tracker.

\subsubsection{Wide Angle Bremsstrahlung}

Dark-strahlung is hidden by a large flow of electron-positron pairs coming from the beam backgrounds, as discussed in sec.5.2. These include the bremsstrahlung photons produced in the primary target and converted to electron positron pairs in 
the target or in layers of the SVT. These events may pass the trigger requirements, mimicking the signal through one of the schemes in Fig. 7.2.9. The bremsstrahlung photon is produced at large angles, such that the converted electron and positron can be detected in one of the halves of the HPS detector, while the beam electron is detected in the opposite half. Pairs formed with the converted positron and the scattered beam electron trigger for HPS signal. To filter out these events, simulations of WAB were performed, studying their properties.

\section{The $D_{0}$ parameter as a wide angle bremsstrahlung discriminator}

The $D_{0}$ parameter of a track is defined as the distance of closest approach of a track to the beam line, as shown in Fig. 7.2.10 (detailed explanations in Ref.[128]). In the analysis of the HPS data-set with the SVT at the $0.5 \mathrm{~mm}$ setting, $D_{0}$ is used to reduce the contamination from WAB events events. The simulations for the $0.5 \mathrm{~mm}$ data showed a shifted $\mathrm{D}_{0}$ distribution for the simulated WAB sample with a missing signal in the first layer of the SVT. The $0.5 \mathrm{~mm}$ real data show the same behavior, motivating the requirement of a signal in the first layer of the SVT to reduce the WAB contamination. For the $1.5 \mathrm{~mm}$ setting, however, the comparison of the distributions of $D_{0}$, shown in Fig.7.2.11 (in particular the black and green lines), indicates that the behavior is different for the $1.5 \mathrm{~mm}$ setting. We conclude from this that WAB events can not be cleanly differentiated with such cut. This can be understood, since in the case of the $1.5 \mathrm{~mm}$ setup, the first layer of the SVT is not as close to the beamline, leading to a significant increase of good events missing the first SVT layer.

\section{Transverse Momentum Asymmetry as WAB discriminator}

The $\Delta P_{t}$ transverse momentum asymmetry of the electron and positron relative to the pair transverse momentum, is defined as:

$$
\Delta P_{t}=\frac{p_{t}\left(e^{-}\right)-p_{t}\left(e^{+}\right)}{p_{t}\left(e^{-}\right)+p_{t}\left(e^{+}\right)}
$$

where $p_{t}$ is the transverse momentum of a particle. It should be non-zero when the electron and the positron don't come from the same vertex, so that it can get rid off events like in Fig. 7.2.9 left. When the detected pair comes from such process, the $\Delta \mathrm{P}_{\mathrm{t}}$ distribution has an asymmetry coming from the energy imbalance between the beam electron and the pair-positron. Based on the simulation for the $0.5 \mathrm{~mm}$ setting, a $\Delta \mathrm{P}_{\mathrm{t}}$ cut has been implemented for the data from the $0.5 \mathrm{~mm}$ setting, reducing the contamination from WAB events [127]. We compare the distributions from the $1.5 \mathrm{~mm}$ data set with the $0.5 \mathrm{~mm}$ data set in Fig. 7.2.12. One can notice that all the distributions on the left plot from the $1.5 \mathrm{~mm}$ data are slightly asymmetric. They keep this trend independently from the selection criteria listed in Fig. 7.2.12. The largest difference between the $0.5 \mathrm{~mm}$ and the $1.5 \mathrm{~mm}$ data is for the bright 
green lines, where the positron has a signal in the second SVT layer but not in the first layer. This asymmetry observed for the $0.5 \mathrm{~mm}$ data is introduced by the WAB contamination and misidentified electron partners. However, the $1.5 \mathrm{~mm}$ distribution does not behave in the same way. Thus, we come to the conclusion that there are less WAB events with the $1.5 \mathrm{~mm}$ setting. This is understandable since the first SVT layer is further from the beam, limiting conversion possibilities and acceptance of WAB events converted in the target.

To summarize the studies of WAB contamination in the $1.5 \mathrm{~mm}$ data set, it follows from the arguments presented above that the WAB events presence is likely less than in the $0.5 \mathrm{~mm}$ data sample. Moreover, the requirement of a hit in the SVT layer one from the positron is not an efficient way to remove the WAB contribution. Finally, the asymmetric $\Delta P_{t}$ distributions are not fully understood in the $1.5 \mathrm{~mm}$ setting, such that further simulation studies in this setting are needed, to understand them.

\subsubsection{Summary of Event Selection}

A summary of the cuts is presented in Tab.7.2.2. To summarize the whole data selection, we show the positron and electron energy distributions after each of these cuts in Fig. 7.2.13. The total energy of the pair and the invariant mass distributions are presented in the same way in Fig. 7.2.14. The invariant mass spectrum colored

\begin{tabular}{|c|c|c|}
\hline Selection for $e^{-} e^{+}$pair & Name & Value \\
\hline \hline Track and cluster cuts & "good particle" & passed cuts in Tab.7.2.1 \\
\hline Matching $\left(e_{\text {cluster }}^{-}-e_{\text {cluster }}^{+}\right)$timing & " $\Delta T_{\text {ecal }}$ " & $\mathrm{t}_{\text {mean }} \pm 3 \sigma\left(t_{\text {mean }}=0.044, \sigma=0.57\right)$ \\
\hline Removing full energy electron & "Coulomb electron" & $E_{e^{-}}<0.85 \mathrm{GeV}$ \\
\hline Reducing Bethe-Heitler background & "Radiative" & $E_{e^{-}}+E_{e}+>0.8 \mathrm{GeV}$ \\
\hline Interaction vertex with the best $\chi^{2}$ & "best Vertex" & $\chi_{\text {min }}^{2}<20$ \\
\hline
\end{tabular}

Table 7.2.2.: Selection criteria applied for pair selection. The second column is the name for each cut referred to in the plots of Fig.7.2.13, Fig. 7.2.14 and in Tab. 7.2.3.

in orange is the final sample to be analyzed for a potential dark photon signal. To conclude this part of the data analysis, the survival rate ${ }^{1}$ of the $\gamma^{\prime}$ candidates from real data are summarized in Tab. 7.2.3. The survival rate of triggered events for the dark photon candidates from the real data is $0.66 \%$.

\footnotetext{
${ }^{1}$ The number of dark photon candidates after each cut.
} 


\begin{tabular}{cc}
\hline Selection for $e^{-} e^{+}$pair & $\#$ of $\gamma^{\prime}$ Candidates From Data \\
\hline \hline All events & $35.609 .180(100 \%)$ \\
\hline \hline All V0 candidates & $6.614 .031(18.6 \%)$ \\
\hline \hline ECal Module & $2.703 .524(7.59 \%)$ \\
\hline \hline Cluster Minimum Energy & $2.698 .866(7.58 \%)$ \\
\hline \hline Geed Hit Timing Window & $2.478 .992(6.96 \%)$ \\
\hline \hline Good particle & $760.180(2.13 \%)$ \\
\hline \hline Coulomb electron & $611.916(1.72 \%)$ \\
\hline \hline Radiative & $587.617(1.65 \%)$ \\
\hline \hline Best Vertex & $298.915(0.84 \%)$ \\
\hline \hline 3. The & $235.243(0.66 \%)$ \\
\hline \hline
\end{tabular}

Table 7.2.3.: The survival rate of $\gamma^{\prime}$ candidates for real data passing through the selection cuts.

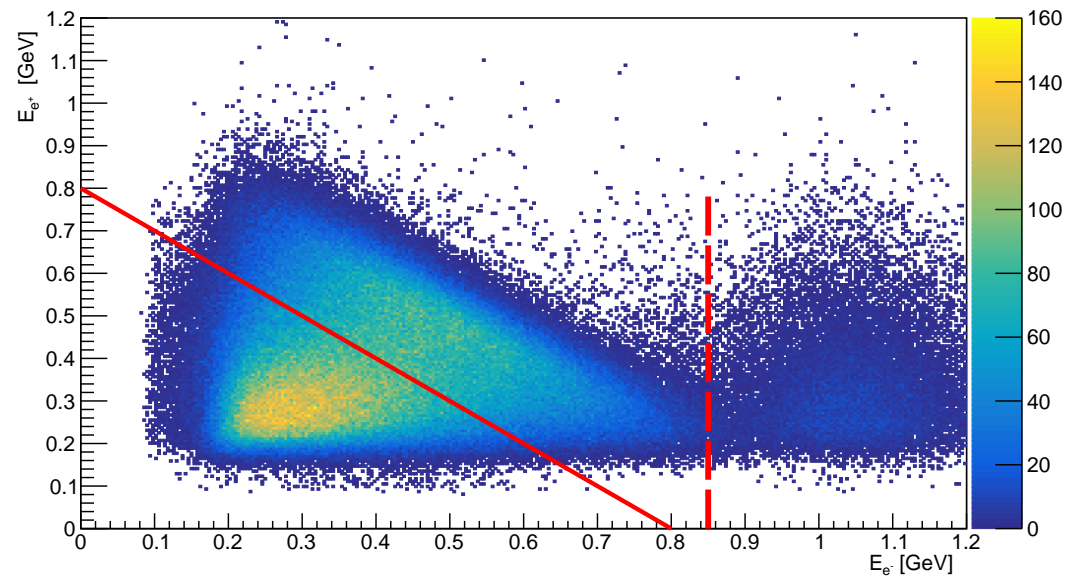

Figure 7.2.4.: The positron energy vs electron energy, where the red dashed line corresponds to the cut on the electron energy $\left(E_{e^{-}}<0.85 \mathrm{GeV}\right)$ to remove the full energy electrons. The red solid line illustrates the radiative cut $\left(E_{e^{-}}+E_{e^{+}}>\right.$ $0.8 \mathrm{GeV})$, aimed at reducing the QED background from the Bethe-Heitler process. 


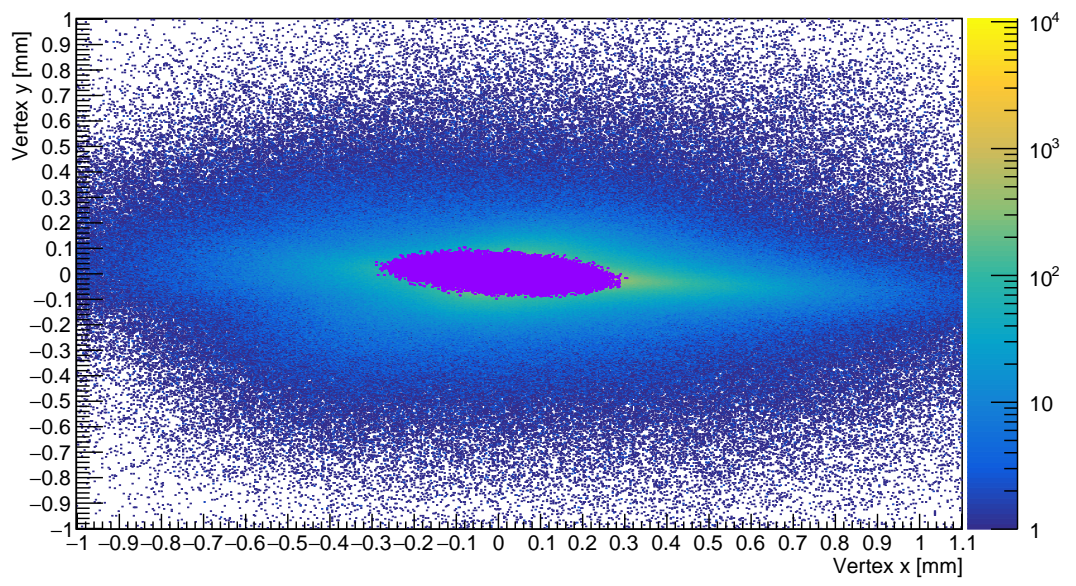

Figure 7.2.5.: $\mathrm{X}$ and $\mathrm{Y}$ position of the interaction vertices at the target for all pairs and for single pairs which passed the cuts, per event (overlaid in purple).

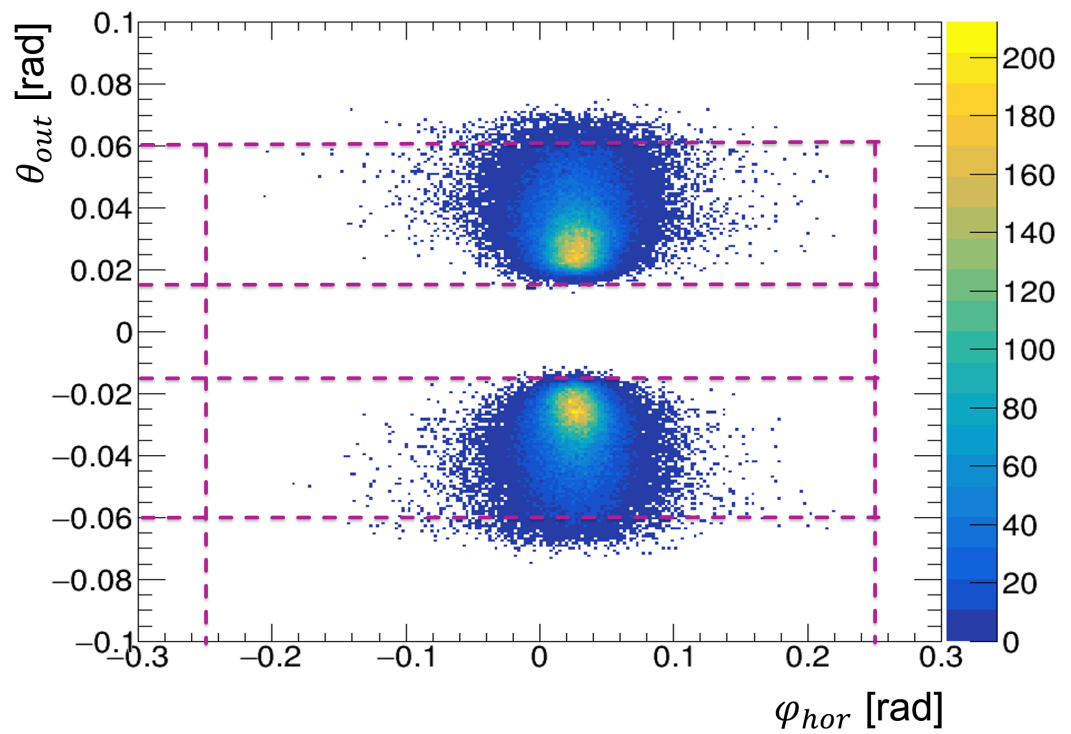

Figure 7.2.6.: Event distribution in the out-of-plane angle $\theta_{\text {out }}$ as a function of the horizontal $\varphi_{\text {hor }}$ angle of the electrons and the positrons. The purple lines represent the acceptance lines used in the background simulations for the calculations with the MC numerical integration (BVMC) described in sec. 5.3.4. 

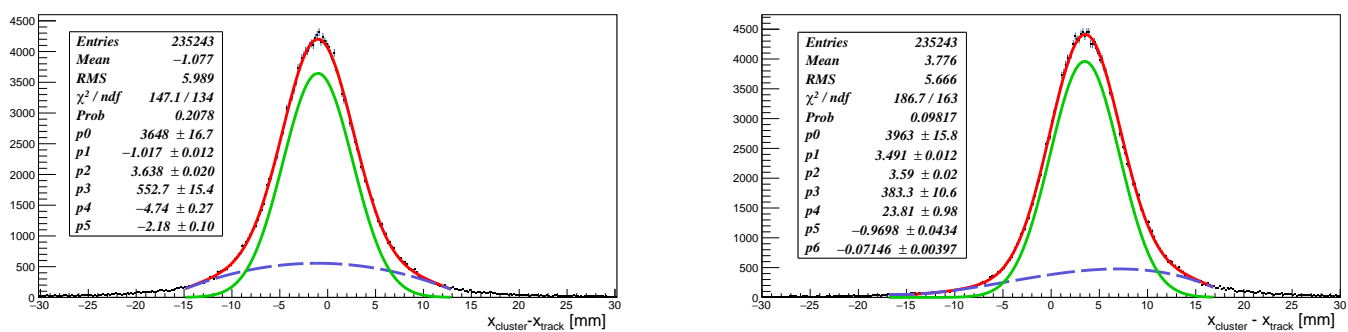

Figure 7.2.7.: The difference of particle position along the $X$-axis in the ECal, between the cluster and the track measurements, for the electron (left) and for the positron (right). The red solid line is a fit using a Gaussian (green line - p0, p1, p2 parameters) and a polynomial function (blue dashed line).
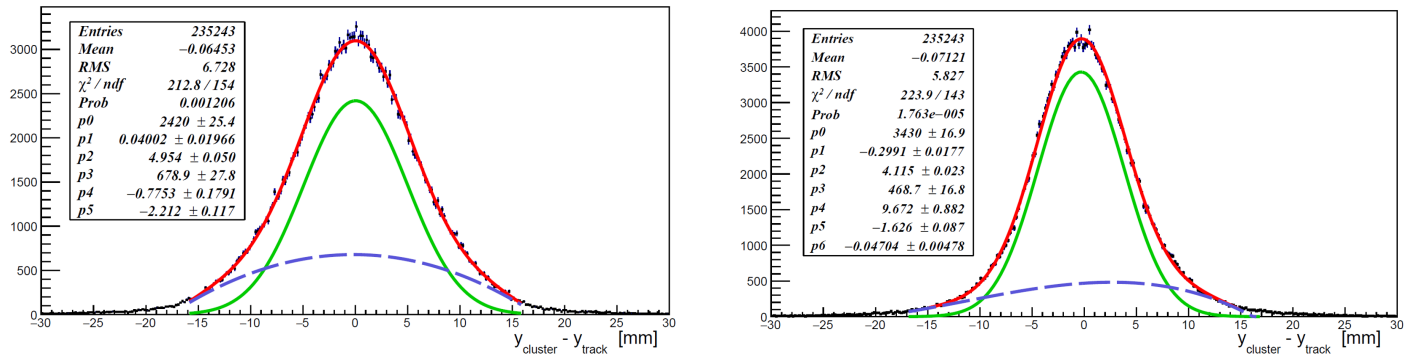

Figure 7.2.8.: The difference of particle position along the $Y$-axis in the ECal between cluster and the track measurements, for the electron (left) and for the positron (right). The red solid line is a fit using a Gaussian (green line - p0, p1, p2 parameters) and a polynomial function (blue dashed line).

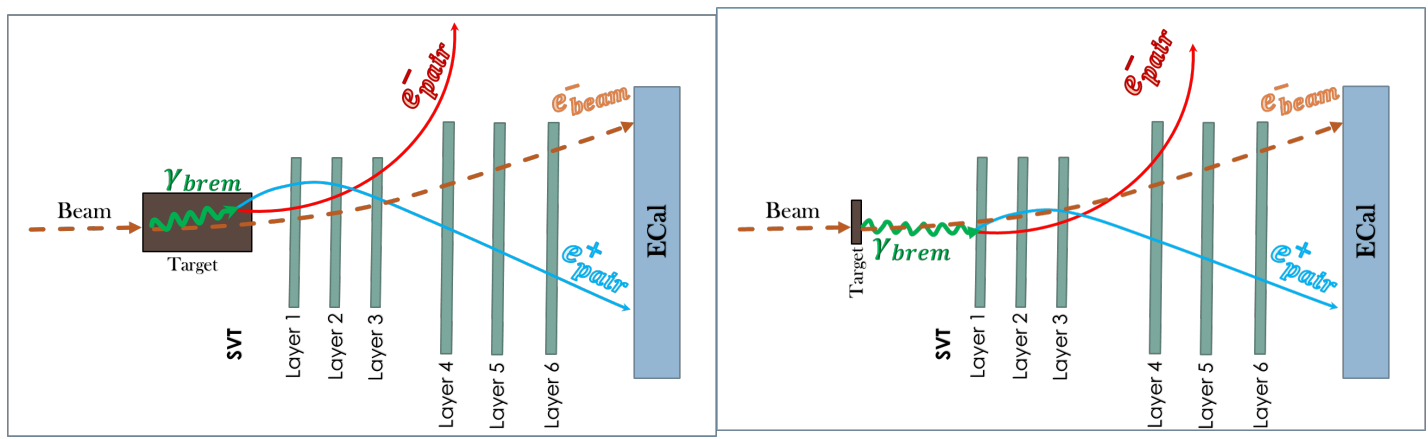

Figure 7.2.9.: Illustration of WAB conversion in the target (on the left) and in the SVT layer 1 (on the right). In both cases the recovered electron-positron pair contains the beam electron, while the pair electron is scattered at a large angle. 


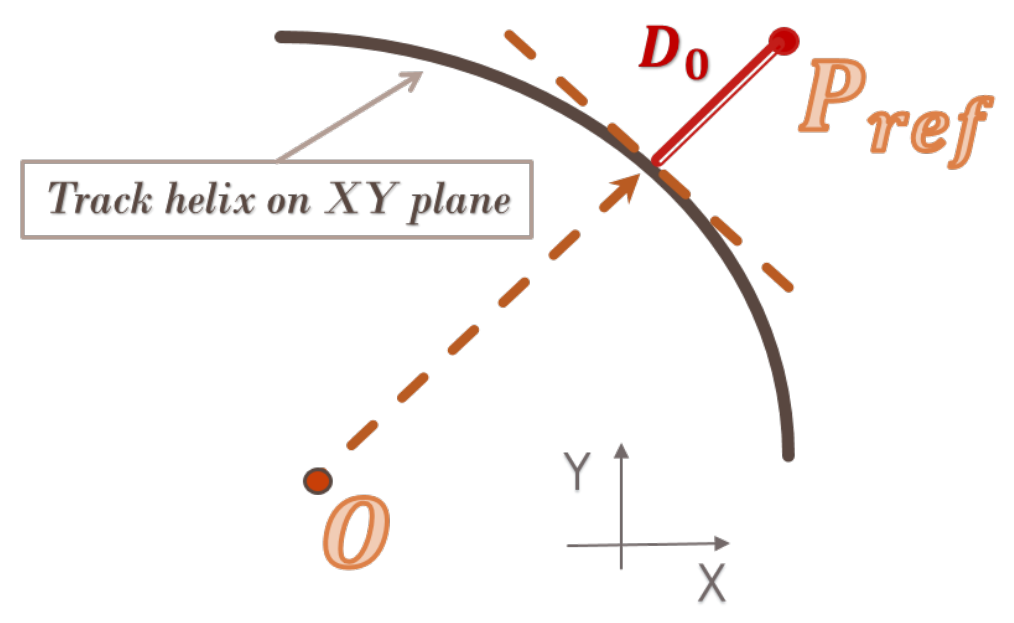

Figure 7.2.10.: Schematic illustration of the $D_{0}$ parameter of a track, defined as the distance of the closest approach to the track helix on the $X Y$ plane relative to the reference point $P_{\text {ref }}$ (beam line for HPS).
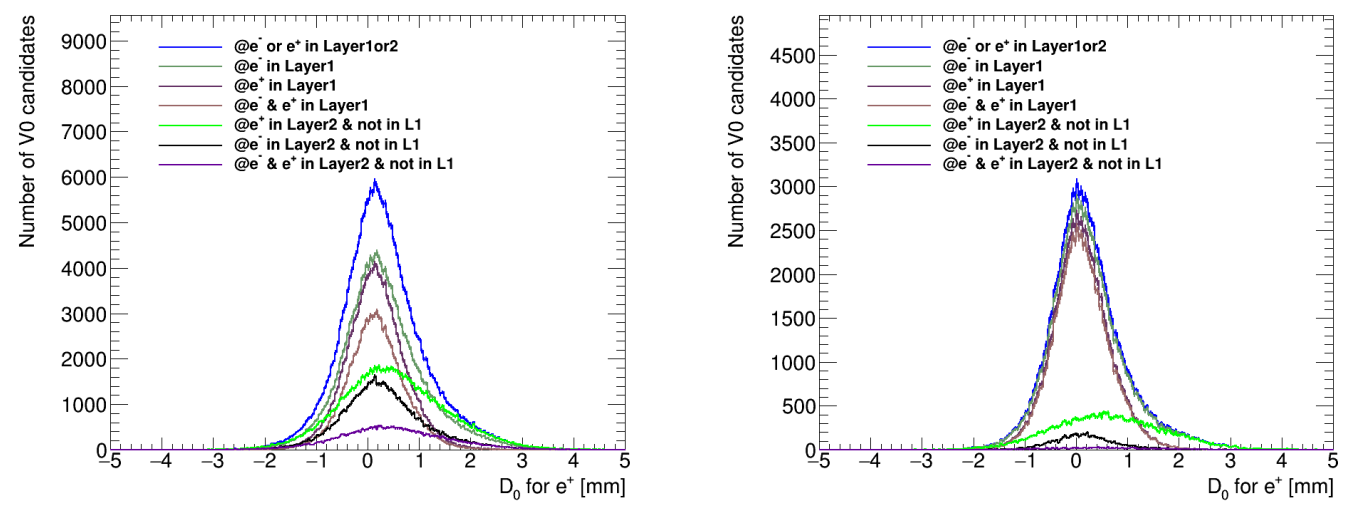

Figure 7.2.11.: The distribution of the $D_{0}$ parameter for the pair positron. On the left, the data with the $1.5 \mathrm{~mm}$ setting; on the right, the data with the $0.5 \mathrm{~mm}$ setting. Each color on the plots corresponds to the condition described in the legend about the status of the electron and positron in the first two layers of the SVT. 

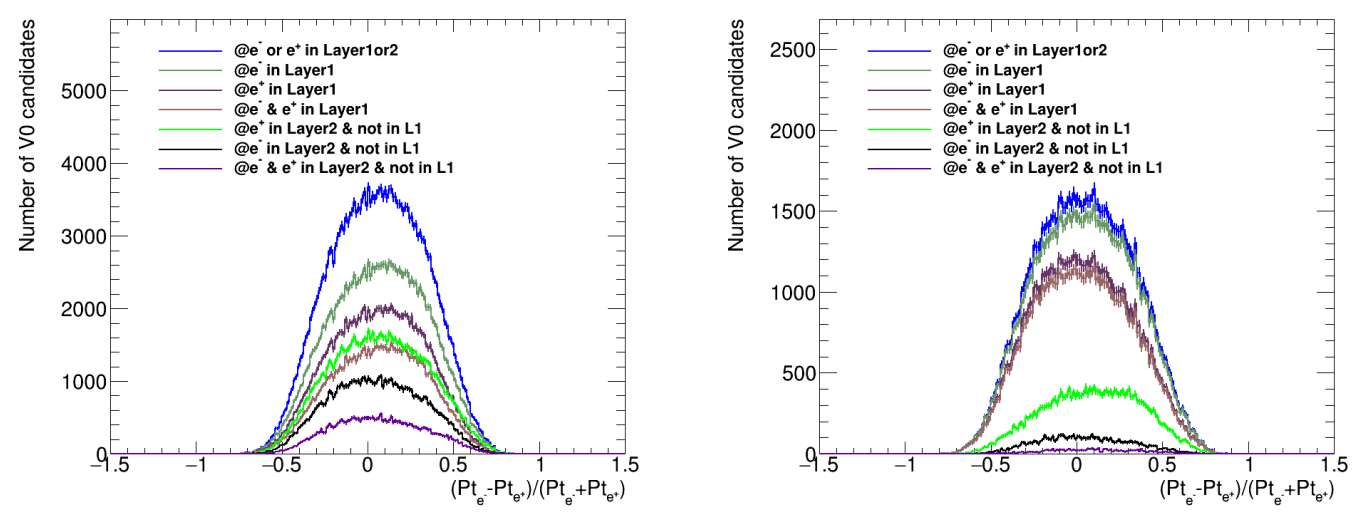

Figure 7.2.12.: The distribution of $\Delta P_{t}$. On the left, the data with the $1.5 \mathrm{~mm}$ setting; on the right, the data with the $0.5 \mathrm{~mm}$ setting. Each color on the plots corresponds to the condition described in the legend about the status of the electron and positron in the first two layers of the SVT. 

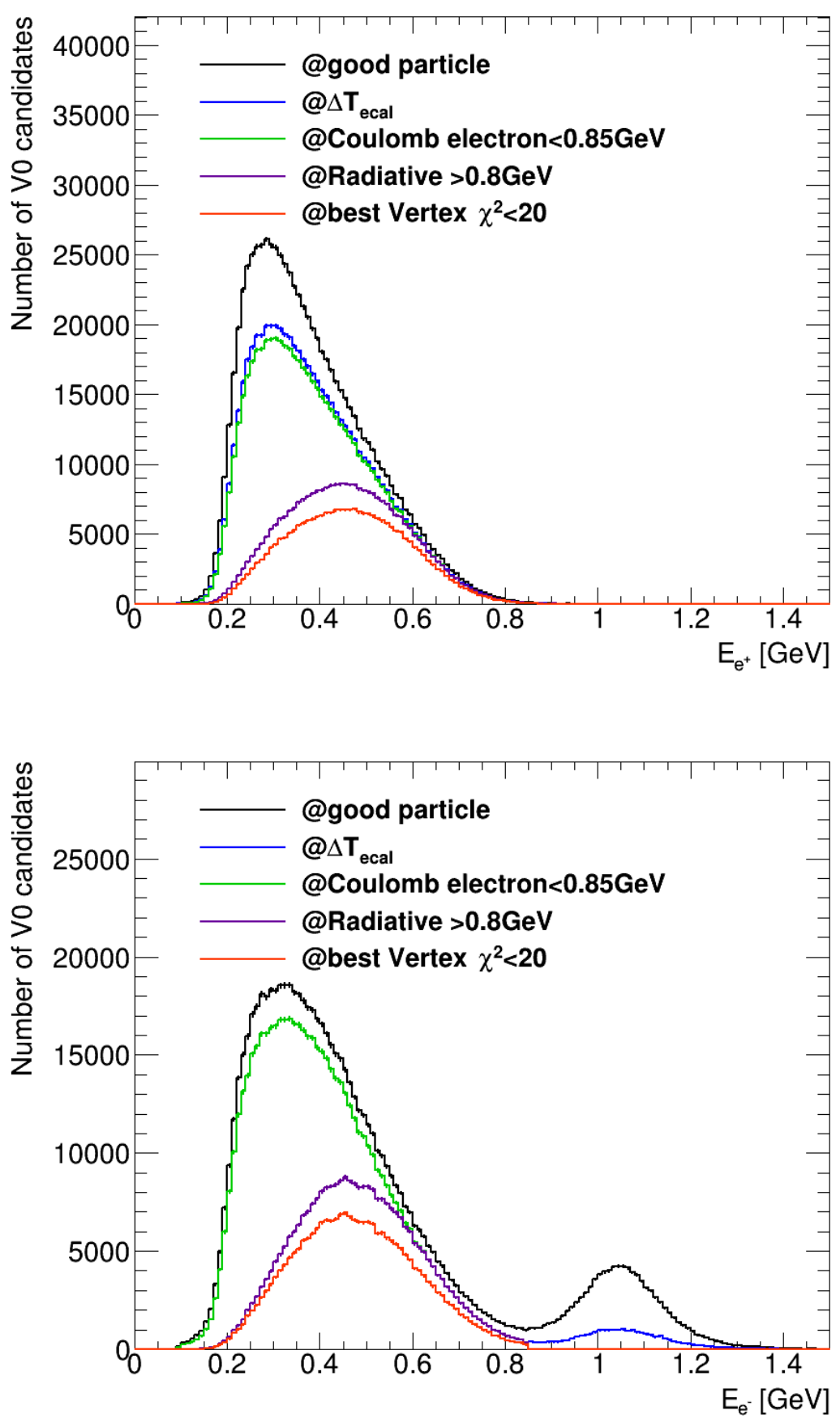

Figure 7.2.13.: Energy distribution of the positron (top) and of the electron (bottom), where each line corresponds to a cut indicated in the legend and described in Tab. 7.2.2. 

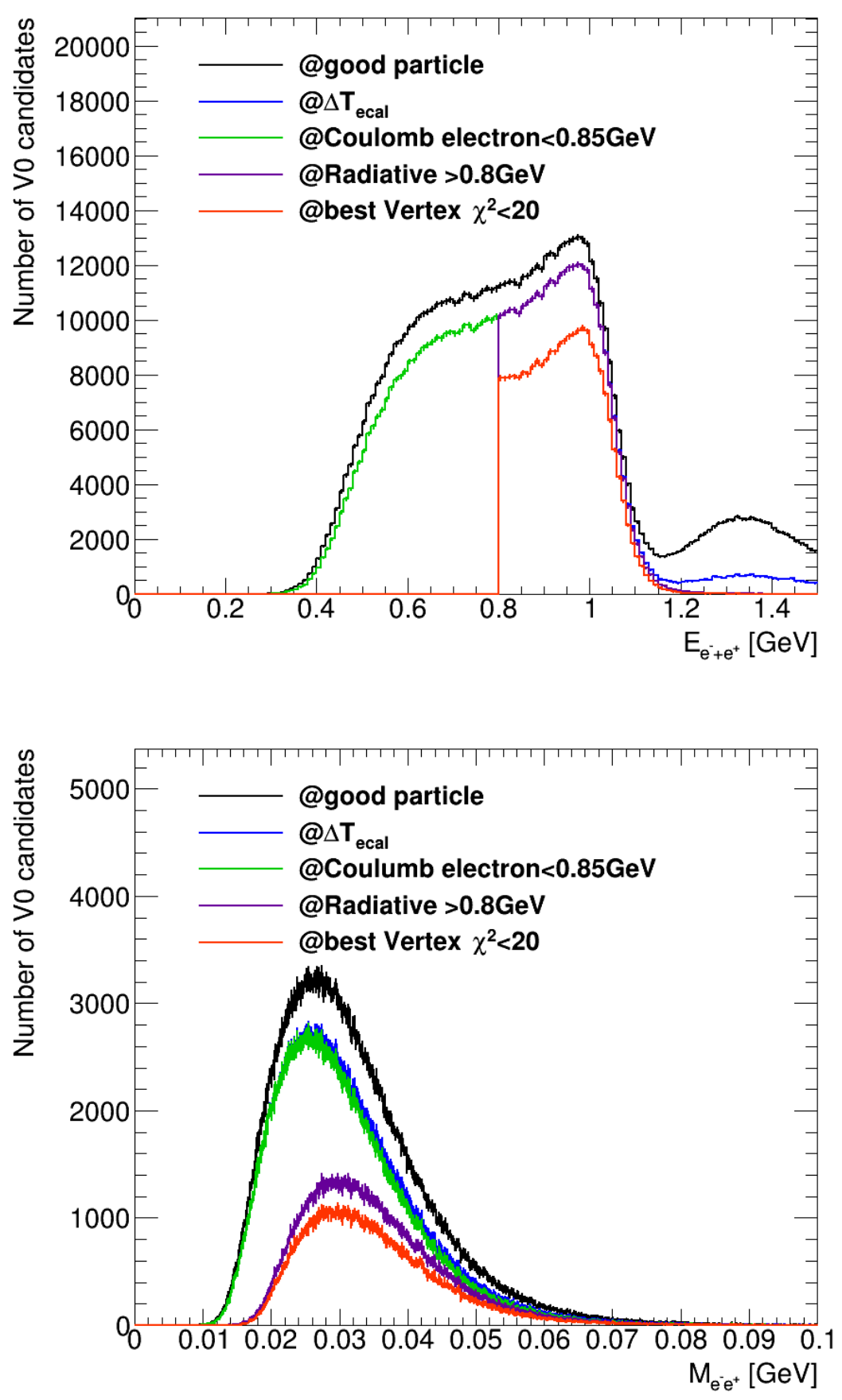

Figure 7.2.14.: Pair energy sum (top) and invariant mass (bottom) distribution of the pair, where each line corresponds to a cut indicated in the legend and described in Tab. 7.2.2. 


\subsection{Statistical Analysis for Resonance Search}

The resonance search is done using statistical data analysis methods. We perform a discovery test by looking for the presence of a new signal in the invariant mass spectrum and then, if it is negative, determine the associated exclusion limits. In both steps, the data are modeled and hypothesis tests are carried out. The statistical analysis presented in this section are based on the theoretical framework developed by Cowan et al. [129].

We have adopted here a frequentist approach, meaning that the probability is interpreted as the limit of the frequency of different outcomes. Then the hypothesis test can be interpreted as the estimate of the probability of the data given the model, without assuming any prior belief in this model, in opposition to the Bayesian approach.

\subsubsection{The Model}

The first step of the analysis is to build a model describing the data. It is a function chosen to describe the invariant mass distribution over its full range. After trying many different functions, the best one proved to be a modified version of the socalled Bukin probability density function (p.d.f.) [130]. The function is based on the convolution of a Gaussian and exponential functions. It has in total five parameters, $\mu_{B}, \sigma_{B}, \rho_{1}, \rho_{2}, \nu$. The first two parameters, $\mu_{B}$ and $\sigma_{B}$, are respectively the peak position and the width of the distribution, while $\rho_{1}$ is characterizing the left tail and $\rho_{2}$ is the parameter describing the right tail. The remaining parameter, $\nu$, is the peak asymmetry parameter. The model was constructed based on the RooFit [131] implementation of the Bukin function, RooAbsPdf::RooBukinPdf. To adapt the function to the shape of the real data distribution, we give it extra flexibility by making the parameters dependent on the invariant mass. Studying the pulls ${ }^{2}$ of the fit results, it was found that the $\mu_{B}\left(M_{e^{+} e^{-}}\right)$and $\sigma_{B}\left(M_{e^{+} e^{-}}\right)$parameters are the least sensitive to the invariant mass dependent modification and do not improve the fit results noticeably. Therefore, only the other three parameters use a dependence on the invariant mass $\left(M_{e^{+} e^{-}}\right)$:

$$
\begin{aligned}
\nu & =k_{0}^{\nu}+k_{1}^{\nu} \times M_{e^{+} e^{-}} \\
\rho_{1} & =k_{0}^{\rho_{1}}+k_{1}^{\rho_{1}} \times M_{e^{+} e^{-}} \\
\rho_{2} & =k_{0}^{\rho_{2}}+k_{1}^{\rho_{2}} \times M_{e^{+} e^{-}}
\end{aligned}
$$

Other modifications were considered as well, but the first order polynomial form proved itself best in terms of minimizing and stabilizing the pulls with the least parameters. This function describes the background in our model. The results

\footnotetext{
${ }^{2}$ The pull is defined for each $y$ bin as the difference between the measured value and the true value from the fit function, divided by the measurement error: $p u l l=\frac{y_{\text {fit }}-y_{\text {true }}}{y_{\text {error }}}$.
} 
of a binned likelihood fit of a data sample with the background function and the associated pulls are plotted in Fig. 7.3.1. The signal in the model is represented with a Gaussian p.d.f.. The width of the signal is calculated from the mass resolution of the detector obtained from simulated $\gamma^{\prime}$ data sample [127]

$$
\sigma=-6.166 M_{e^{+} e^{-}}^{3}+0.9069 M_{e^{+} e^{-}}^{2}-0.00297 M_{e^{+} e^{-}}+0.000579,
$$

where $\sigma$ is the Gaussian width and $M_{e^{+} e^{-}}$is the invariant mass, both in $[\mathrm{GeV}]$ units.
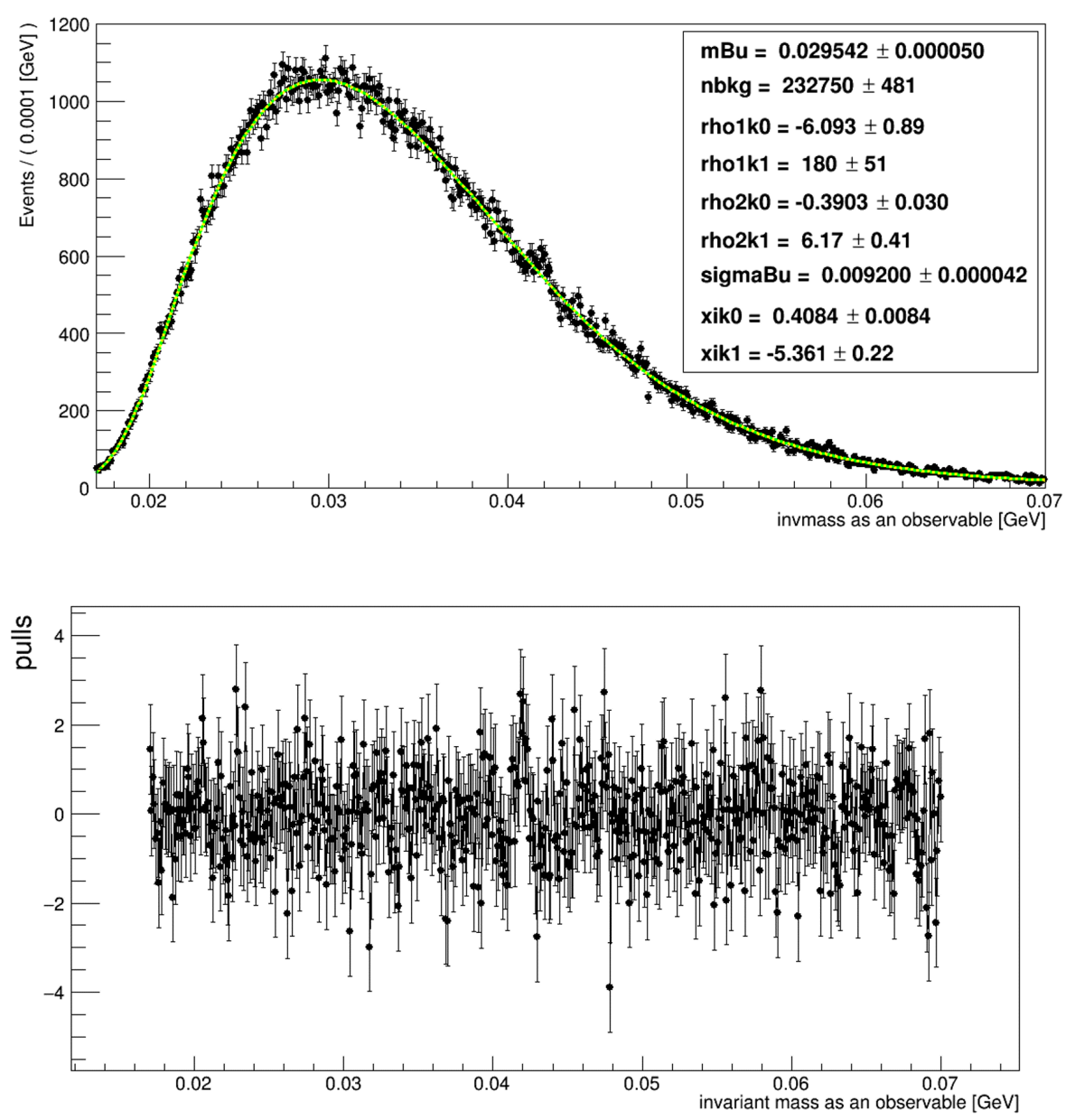

Figure 7.3.1.: Invariant mass distribution fit with the Bukin probability density function (top) and the pulls (bottom) from data.

\subsubsection{Hypothesis Test}

To claim the discovery of a new phenomenon, one needs to show that the known physics processes cannot fully describe the data, assuming all detector effects are 
accounted for in the model. The hypothesis test is a statistical test estimating the compatibility of the data with the model. The data are compared against a model with a signal equal to 0 , called null hypothesis, $H_{0}$. Data are then compared with an $H_{1}$ alternative hypothesis, including the signal.

As discussed in the previous section, the model is constructed from a probability density functions of Gaussian, describing the signal and modified Bukin function, describing the background. The signal strength in the model is characterized by a parameter $N_{s}$, so that the number of signal events in a bin is $N_{s} \times p . d$.f.Gaus $(x)$. The total number of events in a bin, then, is the sum of the signal events and background events:

$$
N_{s} \times p . d . f \cdot \text { Gaus }(x)+N_{b} \times p . d . f \cdot \text { Bukin }(x),
$$

where the $N_{b}$ parameter, weighted by the background p.d.f, quantifies the number of background events. Note that the signal strength can be negative, allowing for downward fluctuations. This special case will be discussed later. $N_{s}$ is the parameter of interest (POI) and the rest of the parameters of the model, including $N_{b}$, are the nuisance parameters.

With a purpose of discovery, $H_{0}$ is the background-only hypothesis. Therefore, the signal strength parameter is set to $0, N_{s}=0$. The null hypothesis is constructed so that the alternative hypothesis reduces to $H_{0}$ with $N_{s}=0$, without additional free parameters for the signal p.d.f.. This means that the mean and the $\sigma$ of the Gaussian in the model have fixed values for a given test. When calculating the exclusion limits, we do the opposite. $H_{1}$ is taken as the null hypothesis and is tested against $H_{0}$ as the alternative hypothesis. This way, the fluctuations in the data are not considered as an indication of a deviation from the background-only model, thus testing the exclusion of a signal rather than its presence.

The hypothesis test is performed for each conjectured $\gamma^{\prime}$ mass in the range of invariant mass. To do so, we divide the invariant mass range in 100 intervals, and test the central value of each interval for a signal. This value is set as the Gaussian mean.

\subsection{3. $\mathrm{p}$-value}

The agreement of the data with the hypothesis is quantified by the p-value, the probability of having data agreeing or being incompatible with the predictions of the hypothesis. The profile likelihood ratio can be used as a measure of this incompatibility, as will be discussed in the next section. The p-value is defined as the integral of the upper tail of the standard normal distribution with $Z$ standard deviations from the mean. This is illustrated by the right plot in Fig. 7.3.2. The relation of the p-value with the $Z$ value of a standard Gaussian is

$$
Z=\Phi^{-1}(1-p)
$$


where $\Phi^{-1}$ is the quantile ${ }^{3}$ of the Gaussian. The threshold for the p-value in particle physics to claim a discovery is traditionally $Z=5$ standard deviations. The p-value can be defined both as the one-sided and as the two-sided integral of the standard Gaussian, illustrated respectively on the left and right plots of Fig. 7.3.2. Here, we use the one-sided definition, where $Z=5$ corresponds to $p=2.87 \times 10^{-7}$. The choice of this value is mainly motivated by the experience of statistical analysis in particle physics, as many $3 \sigma-4 \sigma$ claims were found to be statistical fluctuations, ultimately disappearing with more data. In practice, the threshold for $Z$ should rather be selected circumstantially, depending on the tested hypothesis [132], since as advises Carl Sagan, "Extraordinary claims require extraordinary evidence". In particular, p-values carry the impact of systematic errors and look elsewhere effect (LEE) that vary from one experiment to another. The LEE accounts for finding the signal in the invariant mass distribution at other than the hypothesized mass. The p-value corrected for the LEE estimates the global p-value. Additionally, there is the "experimenter's bias" coming from the expectations of the researcher analyzing the data, as discussed in $[132,133]$. Often, as for the present work, a blind analysis method is exploited to account for this [133]. In the HPS collaboration, and therefore in this thesis, we only analyze a fraction of the data in order to blind the analysis. The analysis is only performed on the full data once the collaboration considers the analysis to be final.
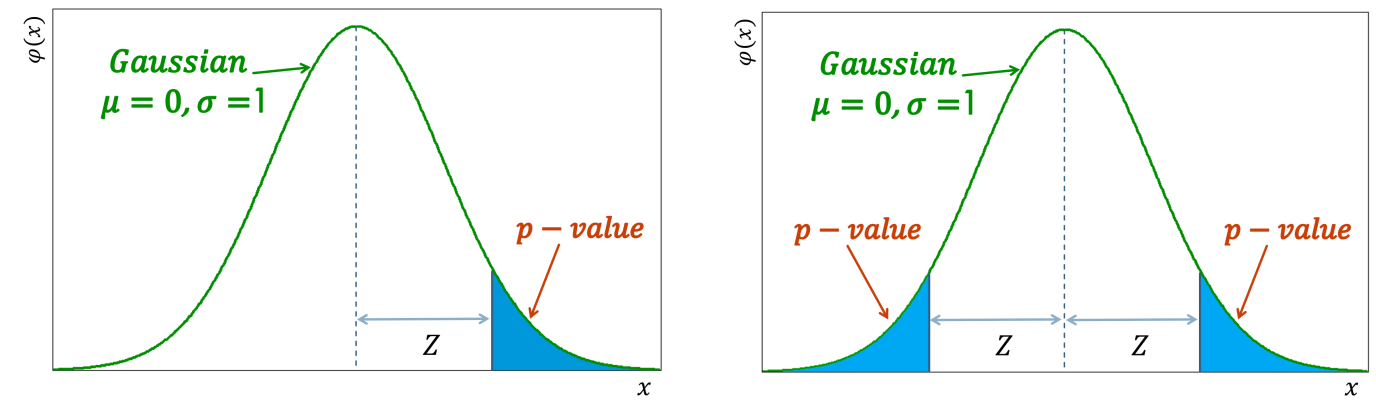

Figure 7.3.2.: The p-value illustrated on a standard normal distribution, as the integral from $Z$ standard deviations away from the Gaussian mean $\mu$. One-sided (left) and two-sided (right) definitions of the p-value are shown.

To obtain the p-value, the ratio of maximum likelihood functions, the profile likelihood is calculated for each hypothesis test, as detailed below.

\footnotetext{
${ }^{3}$ Inverse of the cumulative distribution of a Gaussian.
} 


\subsubsection{Profile Likelihood Ratio}

The profile likelihood ratio is defined as [129],

$$
\lambda(\mu)=\frac{L(\mu, \hat{\hat{\theta}})}{L(\hat{\mu}, \hat{\theta})}
$$

where $L$ is the likelihood constructed as a product of the Poisson probabilities of all data bins, where the POI is noted $\mu$ and $\theta$ is the set of the nuisance parameters [129]:

$$
L(\mu, \theta)=\prod_{j=1}^{N} \frac{\left(\mu \times s_{j}+b_{j}\right)^{n_{j}}}{n_{j} !} e^{-\left(\mu \times s_{j}+b_{j}\right)} .
$$

Here, $N$ is the number of bins and $n_{j}$ is the observed number of events in the bin $j . s_{j}$ and $b_{j}$ are respectively the number of signal and background events in a bin, defined as:

$$
\begin{aligned}
& s_{j}=s_{\text {tot }} \int_{b i n j} f_{s}\left(x, \theta_{s}\right) d x, \\
& b_{j}=b_{\text {tot }} \int_{b i n j} f_{b}\left(x, \theta_{b}\right) d x .
\end{aligned}
$$

where $f_{s}\left(x, \theta_{s}\right)$ and $f_{b}\left(x, \theta_{b}\right)$ are the p.d.f. of the signal and of the background. $s_{t o t}$ and $b_{t o t}$ are the means of the number of events of the signal and of the background respectively.

In eq.(7.3.7), the numerator $L(\mu, \hat{\hat{\theta}})$ is the maximum likelihood at a fixed POI value, $\mu$. $\hat{\hat{\theta}}$ is called the conditional maximum-likelihood estimator of $\theta$. The denominator $L(\hat{\mu}, \hat{\theta})$ maximizes all the parameters of the likelihood fit, i.e. both the $\hat{\theta}$ nuisance parameters and the POI $\hat{\mu}$, which are free parameters. The $\hat{\mu}$ and $\hat{\theta}$ parameters are then referred to as the unconditional maximum-likelihood or best estimators of the model parameters $\mu$ and $\theta$. The graphical interpretation of the profile likelihood ratio is illustrated in Fig. 7.3.3.

\subsubsection{Test Statistics}

The profile likelihood is by definition in the range $0<\lambda(\mu)<1$. High values indicate agreement with the null hypothesis and low values a rejection of the null hypothesis. A more convenient quantity to describe the statistical test, is defined from the profile likelihood ratio:

$$
t_{\mu}=-2 \ln \lambda(\mu),
$$




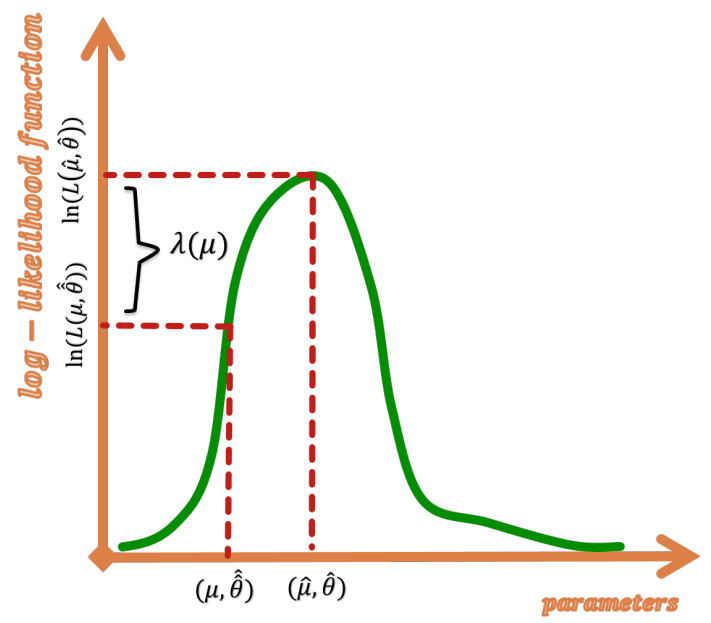

Figure 7.3.3.: The graphical representation of the profile likelihood ratio. On the $Y$ - axis is the log-likelihood function for data given a model with parameters $(\mu, \theta)$. On the $X-$ axis are the parameters of the model. The profile likelihood ratio is the difference between the log-likelihood function at $\mu=0$ and with nuisance parameters $\hat{\hat{\theta}}$ and the log-likelihood function at the maximum with parameters $(\hat{\mu}, \hat{\theta})$.

where $t_{\mu}$ is the test statistics. In this formulation, the large values of $t_{\mu}$ correspond to a disagreement between the hypothesis and the data as a function of $\mu$. The test statistics is related to the p-value with the following expression [129]:

$$
p_{\mu}=\int_{t_{\mu}, o b s}^{\infty} f\left(t_{\mu} \mid \mu\right) d t_{\mu}
$$

where $t_{\mu, o b s}$ is the test statistics obtained from the data with a maximum likelihood fit, calculating the conditional and unconditional maximum-likelihood estimators $L(\mu, \hat{\hat{\theta}})$ and $L(\hat{\mu}, \hat{\theta})$. Furthermore, $f\left(t_{\mu} \mid \mu\right)$ is the p.d.f. of the test statistics $t_{\mu}$ for the hypothesized $\mu$ value. The relation between the test statistics p-value defined from eq.(7.3.12) and the definition using the standard normal distribution $Z$ from sec. 7.3.3 is illustrated in Fig. 7.3.2 and in Fig. 7.3.4.

According to Wilks' theorem [134], if the following criteria are met:

- $H_{1}$ and $H_{0}$ are nested (i.e. $H_{1}$ can be reduced to $H_{0}$ without leaving any undefined parameters),

- $H_{0}$ is true,

- the additional free parameters in $H_{1}$ are defined and not at the boundary of their limits,

- there is a large sample of an observable of size $N$, 


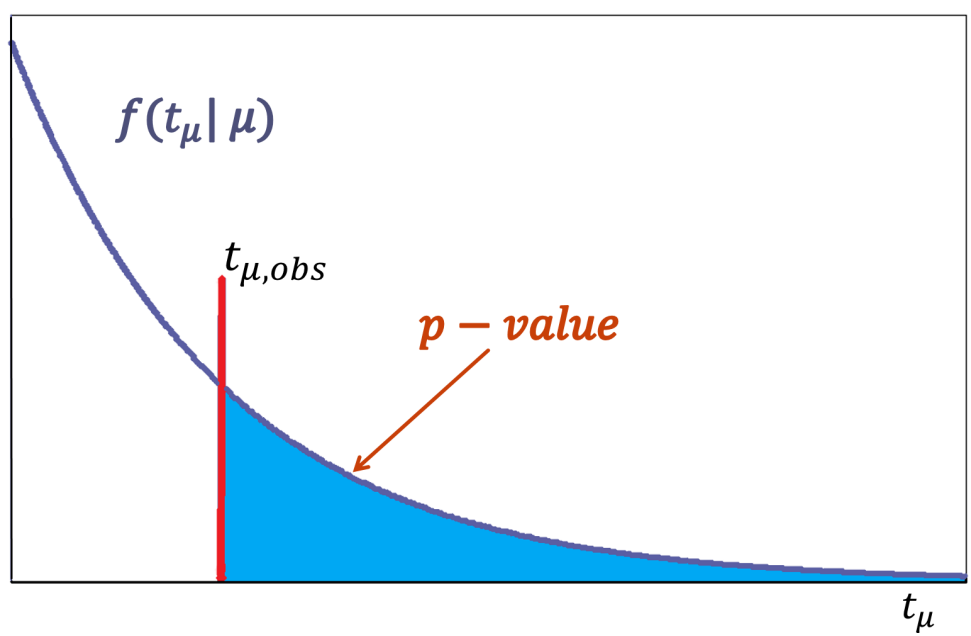

Figure 7.3.4.: The test statistics distribution $f\left(t_{\mu} \mid \mu\right)$ and the corresponding representation of the p-value as its integral from $t_{\mu, o b s}$ to $\infty$, where $t_{\mu, o b s}$ is the observed test statistics and $\mu$ is the signal strength.

then the test statistics p.d.f. $f\left(t_{\mu}\right)$ is a chi-squared distribution of order $k$, at the limit of $N \rightarrow \infty$, where $k$ is the difference between the number of free parameters of unconditional and conditional likelihood estimators. The model introduced in the previous section satisfies these conditions. This theorem was generalized including the $H_{1}$ hypothesis by Wald [135]. He showed that for a sufficiently large $N$, the $t_{\mu}=-2 \ln \lambda(\mu)$ is distributed according to the non-central chi-squared distribution with $k$ degrees of freedom. Then for the test statistics [129]:

$$
t_{\mu}=-2 \ln (\lambda(\mu))=\frac{(\mu-\hat{\mu})^{2}}{\sigma^{2}}+O(1 / \sqrt{N})
$$

where $\hat{\mu}$ has a Gaussian distribution with a mean $\mu^{\prime}$ and standard deviation $\sigma$, that can be found from the covariance matrix. The non-centrality parameter is defined as:

$$
\Lambda=\frac{\left(\mu-\mu^{\prime}\right)^{2}}{\sigma^{2}}
$$

In the case $\mu=\mu^{\prime}, \Lambda=0$ and the $f\left(t_{\mu}, \Lambda\right)$ becomes the chi-squared distribution, reducing to Wilks' theorem.

\section{Test statistics for discovery}

For our analysis, we consider only a positive signal in the alternative hypothesis: $\mu>0$. The null hypothesis of a discovery test is constructed assuming no signal, 
$\mu=0$. Following the notation in [129], the test statistics $q_{0}$ is defined from the profile likelihood ratio with $\mu=0$ :

$$
\begin{aligned}
& q_{0}=-2 \ln \lambda(0), \hat{\mu} \geq 0 \\
& q_{0}=0, \hat{\mu}<0
\end{aligned}
$$

This test rejects the background-only hypothesis in the presence of a positive signal $\hat{\mu}>0$. The nuisance parameters add flexibility to the model allowing to fit the downward fluctuations. However, if the best estimator of the signal strength is still negative $\hat{\mu}<0$, the test statistics is $0, q_{0}=0$. The p-value then can be calculated from eq.(7.3.12) with $q_{0}$, where $f\left(q_{0} \mid 0\right)$ is approximated with the sampling distributions described in [129].

\subsubsection{The Discovery Test Results}

The results of the discovery tests from the data are plotted in Fig. 7.3.5. With the data sample we used, the mass range is between $0.018 \mathrm{GeV}$ and $0.0578 \mathrm{GeV}$. The results show small fluctuations along the invariant mass distribution. Note that the LEE is not accounted for in this graph. The $Y$ - axis represents the local p-value ${ }^{4}$. The study of the extended maximum likelihood fits with pseudo-data samples generated from the background-only model showed that the fit is biased at small masses, where the fitting procedure tends to favor the presence of peaks occurring as a result of the unconditional likelihood fit, when all parameters of the model are free. The unconditional extended maximum likelihood fit for about 1000 pseudo-data distributions $^{5}$, generated with the background-only model, is illustrated in Fig. 7.3.6. It was found that the parametrization of the left tail of the invariant mass distribution has problems, since the log-likelihood distributions have a singularity at their minimum for the $k_{0}^{\rho_{1}}$ and $k_{1}^{\rho_{1}}$ parameters. Various other parametrization forms were considered for the whole distribution as well as just for the left tail description. But taking into account the stability of the pulls, a more successful form was not found in the timeline of this work. However, these parameters are fixed during the fitting procedure and do not create problems for the error matrix calculation of the final fit. The fit model minimization package operates in a 9-dimensional parameter phase-space in case of the conditional fitting and a 10-dimensional one in the case of unconditional fitting. Due to the modifications introduced in eq.(7.3.1), (7.3.2) and (7.3.3), the correlations make the global minimum search of the extended maximum likelihood fit non-trivial. The fitting procedure takes care of the correlations optimizing the parameter-space depending on the mass hypothesis. However, it was found that this optimization introduces some instabilities in the final result. In Fig. 7.3.5, there are indications of upward fluctuations at masses in the $0.0325-0.05 \mathrm{GeV}$ range, that

\footnotetext{
${ }^{4}$ In the following, when we refer to the p-value, it is the local p-value, unless mentioned otherwise.

${ }^{5}$ In the following, we will also use "toy-distribution" when referring to these pseudo-data distributions.
} 
can be seen in the invariant mass distribution in Fig. 7.3.1. However, the width of these bumps is much smaller than the mass resolution of the detector, leading to the observed rapid variations. These are random fluctuations that, most likely, will disappear with more statistics.

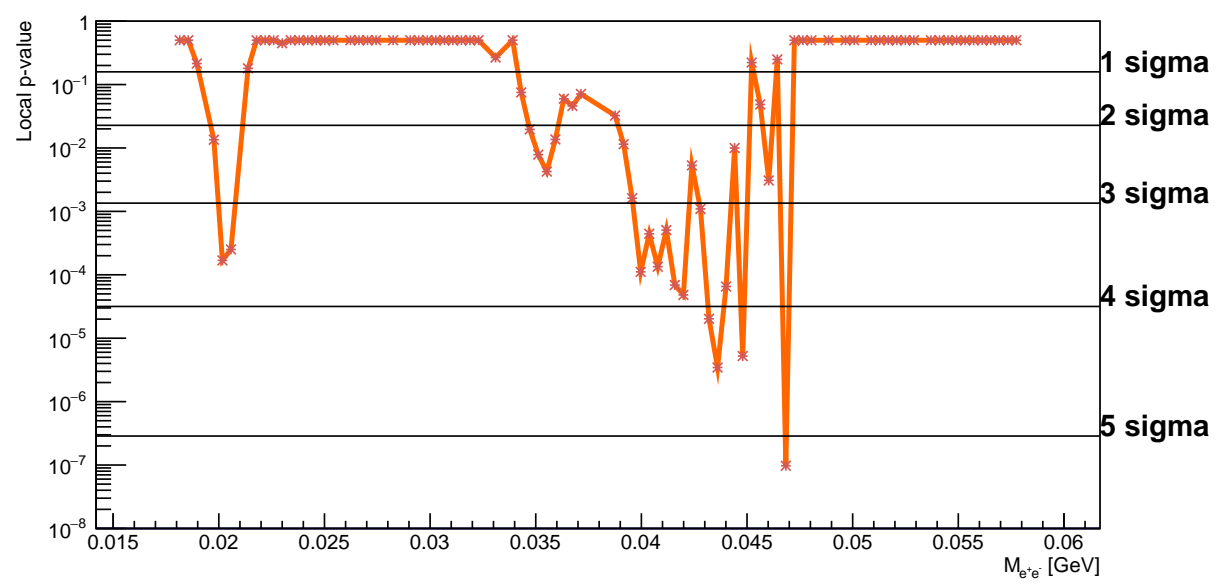

Figure 7.3.5.: The local p-value as a function of the hypothesized $\gamma^{\prime}$ mass from a sample of data.

In Fig. 7.3.7, the mapping between local p-values and global p-values is illustrated for 949 toy-distributions excluding the low mass region $\left(m_{\gamma^{\prime}}>0.023 \mathrm{GeV}\right)$ because of the fit bias discussed before. To obtain this mapping, each of the toy-distributions is generated from the model without a signal and a discovery test is performed using the same analysis tools developed for the real data. The minimum p-value of each distribution is extracted and sorted, pushing forward the smallest p-value obtained from the toys. The local p-value as a function of the normalized index of this sorted set creates the conversion of the local p-value to global p-value. The precision of this conversion is proportional to the number of the toys analyzed, however, we used only 949 toys because of the limited time. We use the mapping in Fig. 7.3.7 to estimate the sizes of the fluctuations at $m_{\gamma^{\prime}}>0.023$ in the data. From the two graphs, one can see that these fluctuations are about $1 \sigma$ or smaller.

\section{Upper Limit Calculation}

To set the upper limits on $\mu$, we want to identify the value of $\mu$ for which the median p-value is 0.05. The upward fluctuations of the data in this case do not indicate an increasing incompatibility with the hypothesized $\mu$ value, and the test statistics is defined to be 0 . Then, the null hypothesis is the hypothesis containing the signal and reduces to the alternative hypothesis for $\mu=0$. 
Assuming the Wald approximation discussed in sec.7.3.5, the median upper limit on $\mu$ at the $(1-\alpha)=95 \%$ confidence level is calculated as [129]:

$$
\mu_{u p}=\hat{\mu}+\sigma \times \Phi^{-1}(1-\alpha) \text {. }
$$

This expression is valid if the $\mu$ signal strength follows a Gaussian distribution with a mean $\mu^{\prime}$ and a standard deviation $\sigma$. To make sure this condition is met, a special Asimov data set is generated for a $\mu^{\prime}=0$ signal strength and $\sigma$ is obtained from a likelihood fit to this data set.

\subsubsection{Asimov Data Set and Variance of $\hat{\mu}$}

The data set name is inspired by the story "Franchise" from the science fiction writer Isaac Asimov, where a single representation of a population is selected as reflecting the vote of the entire electorate. It, much like the hero of "Franchise", is a single data set, constructed so that it reflects the properties of the data population. The Asimov data set is defined so that the estimators for all parameters in a likelihood fit result are equal to their true values [129]. For the discovery test, to extract the p-value of the $H_{1}$ hypothesis, the Asimov data set is generated assuming a signal strength equal to its best estimator $\mu^{\prime}=\hat{\mu}$. For the upper limit, the $H_{1}$ hypothesis is the background-only hypothesis, therefore $\mu^{\prime}=0$. Then, for the profile likelihood ratio:

$$
\lambda_{A}(\mu)=\frac{L_{A}(\mu, \hat{\hat{\theta}})}{L_{A}(\hat{\mu}, \hat{\theta})}=\frac{L_{A}(\mu, \hat{\hat{\theta}})}{L_{A}\left(\mu^{\prime}, \theta\right)} .
$$

Here considering the definition of the data set, the estimators of $\hat{\mu}=\mu^{\prime}$ and $\hat{\theta}=\theta$ have their true value in the denominator.

The standard deviation $\sigma$ can be calculated from the Fisher information matrix ${ }^{6}$ and independently from the variance obtained from $\lambda_{A}(\mu)$. In the first case, the $\sigma^{2}$ variance is the diagonal inverse covariance matrix element, corresponding to the POI. For this, an unconditional fit is performed to the Asimov data set.

In the second case, $\sigma$ is extracted from the Asimov test statistics, $q_{\mu, A}=-2 \ln \lambda_{A}(\mu)$. Taking into account the approximation of eq.(7.3.13), the variance $\sigma_{A}^{2}$ can be estimated as:

$$
\sigma_{A}^{2}=\frac{\left(\mu-\mu^{\prime}\right)^{2}}{q_{\mu, A}}
$$

where $q_{\mu, A}=-2 \ln \lambda_{A}(\mu)$. For the calculations, when one is setting upper limits on $\mu, \mu^{\prime}=0$, the standard deviation equivalent to $\sigma^{2}$ from the covariance matrix is calculated as:

$$
\sigma_{A}=\frac{\mu}{\sqrt{q_{\mu, A}}} .
$$

\footnotetext{
${ }^{6}$ The Fisher matrix is defined as the inverse of the covariance matrix [136].
} 
We have compared the results of these two methods for various $\mu$ values. The results are plotted for one of the hypotheses in Fig. 7.3.8. The green line corresponds to the variance calculated from the covariance matrix element and the red star-points correspond to the estimation of the variance from the Asimov test statistics for different signal strength $\mu$. The two calculations agree within a few percent. The standard deviation $\sigma_{A}$ calculated from eq.(7.3.19) is not defined around $\mu=0$, since the conditional and unconditional likelihood fits become identical and therefore the Asimov test statistics is zero.

\subsubsection{Exclusion Limits}

The one-sided upper value for each hypothesis calculated from eq.(7.3.16) at the 95\% confidence level is plotted in Fig. 7.3.9.

To convert this to exclusion limits for the $\varepsilon$ coupling, the following formula is used $[127,63,112]$ :

$$
\varepsilon^{2}=\frac{\mu_{u p} / M_{\gamma^{\prime}}}{f \Delta B / \Delta m} \times \frac{2 N_{e f f} \alpha}{3 \pi}
$$

where $\mu_{u p}$ is the upper value of signal strength at $95 \%$ confidence level calculated from eq.(7.3.16), $M_{\gamma^{\prime}}$ is the hypothesized mass of the dark photon considered, $f$ is the fraction of the tridents relative to all other events, $N_{e f f}$ is the number of expected decay channels and $N_{\text {eff }}=1$ since in this mass range only the $\gamma^{\prime} \rightarrow e^{+} e^{-}$ decay is available, $\Delta B$ is the number of background events in the $\Delta m$ mass window and $\alpha$ is the fine structure constant. The $f$ ratio is found from the simulations of the $\gamma^{\prime}$ and all background processes of HPS. Here, we use the result of $0.5 \mathrm{~mm}$ data set, since the simulation samples for the $1.5 \mathrm{~mm}$ were not available. The size of the mass window is $\Delta m=2 \times \sigma$, where $\sigma$ is the mass resolution calculated from the eq.(7.3.4). The $\varepsilon^{2}$ coupling constant value estimated from eq.(7.3.20) is plotted as a function of the dark photon mass in Fig.7.3.10. The $\varepsilon^{2}$ coverage has to remain hidden until these results are approved by the HPS collaboration.

As discussed for the discovery test results, the coverage in the mass range is $0.018-$ $0.0578 \mathrm{GeV}$. This is smaller than the initial estimation of the accessible mass range presented in the proposal $0.012-0.1 \mathrm{GeV}$ [63]. As discussed earlier, the acceptance of HPS is smaller in the vertical direction ( $\theta_{\text {out }}$ limits) due to the ECal gap, affecting the low mass range. The difference with the high mass range is mostly due to the low statistics. The mass range with the results of the $0.5 \mathrm{~mm}$ data is from $\sim 0.015 \mathrm{GeV}$ to $\sim 0.06 \mathrm{GeV}$ [127], confirming the observation of the previous sentence, even though lower masses can not be fully recovered due to the ECal gap. 


\subsection{Conclusion}

In this section, we presented our dark photon analysis starting with the event selection and finishing with the statistical analysis of the $e^{+} e^{-}$invariant spectrum. The main features of dark photon production and its dielectron decay were discussed. The selection cuts were established to extract the $e^{+} e^{-}$pairs coming from the $\gamma^{\prime}$ decay. Since the simulated WAB samples were not available for the $1.5 \mathrm{~mm}$ data set, the WAB contamination was studied based on the study of the WAB events with the $0.5 \mathrm{~mm}$ data set. Two quantities for the WAB discrimination in the $0.5 \mathrm{~mm}$ data set were considered to study their behavior in the $1.5 \mathrm{~mm}$ data set. Some features were not confirmed for the latter. The transverse momentum asymmetry parameter distribution showed a shift in comparison with the same distribution in the $0.5 \mathrm{~mm}$ data set. Simulated data samples are necessary to investigate WAB events in the $1.5 \mathrm{~mm}$ data set in more details.

The invariant mass distribution of the $e^{+} e^{-}$pair is extracted after all the event selection criteria. The hypothesis test is performed to scan this invariant mass spectrum and look for a resonance due to a $\gamma^{\prime}$. The local p-value as a function of the hypothesized masses is obtained, showing small fluctuations within its mass coverage $0.018-0.0578 \mathrm{GeV}$. The model is studied in detail to establish the biases coming from the chosen functional form and the fitting procedure.

The local to global p-value conversion is studied with about 1000 toy-distributions. The results were presented excluding the small masses because of the bias issue discussed above. The upper limit at the 95\% confidence level is calculated and converted in the $\varepsilon^{2}$ vs $M_{\gamma^{\prime}}$ phase-space. The coverage in the mass range is within $0.018-0.0578 \mathrm{GeV}$ for the data sample we used.

The analysis of the dark photon search in the $1.5 \mathrm{~mm}$ data set is still ongoing, since some aspects of both the event selection and the statistical analysis are not finalized. In particular, further studies are necessary with simulations to evaluate the WAB contamination in the $1.5 \mathrm{~mm}$ data set. Also, more analyses need to be done for the hypothesis model, the fitting procedure and for the systematical error evaluation. In addition, to translate the upper limit of the signal strength into a coupling-mass phase-space, a radiative trident ratio estimation ( $f$ in eq.(7.3.20)) is also required for the $1.5 \mathrm{~mm}$ data set. However, the analysis framework is established and can be extended for unblinding and finalizing the results. 


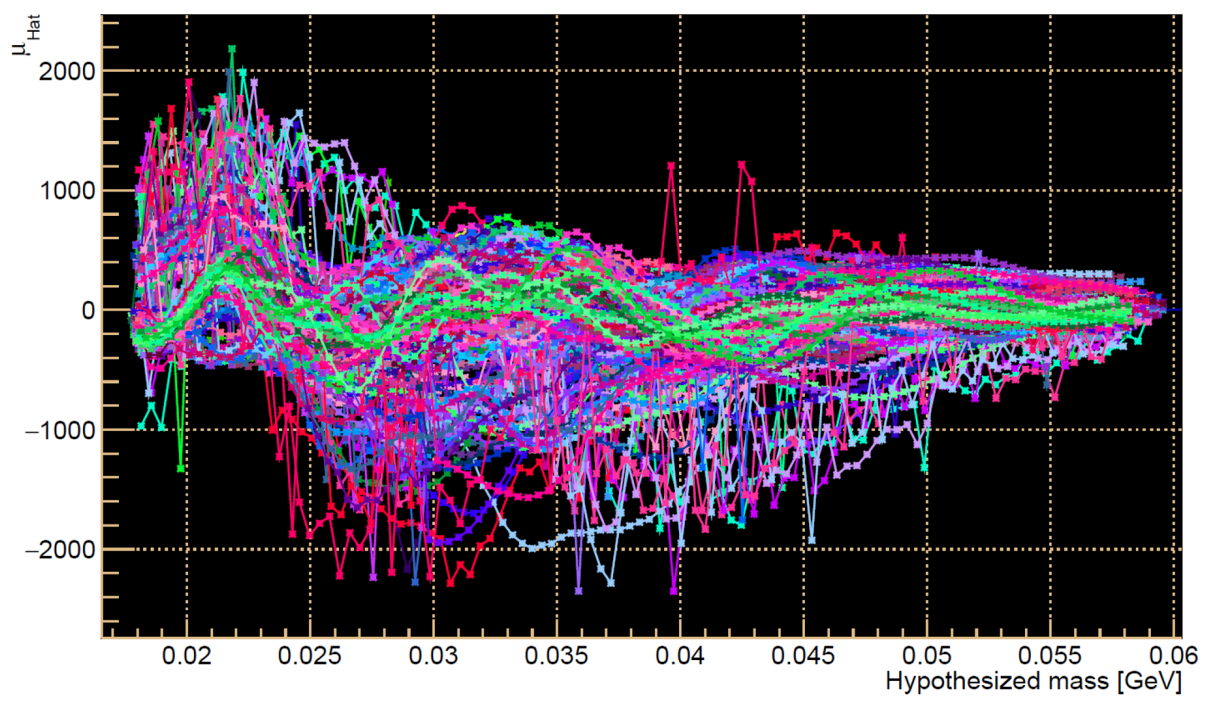

Figure 7.3.6.: The local p-value as a function of the hypothesized $\gamma^{\prime}$ mass from a sample of data.

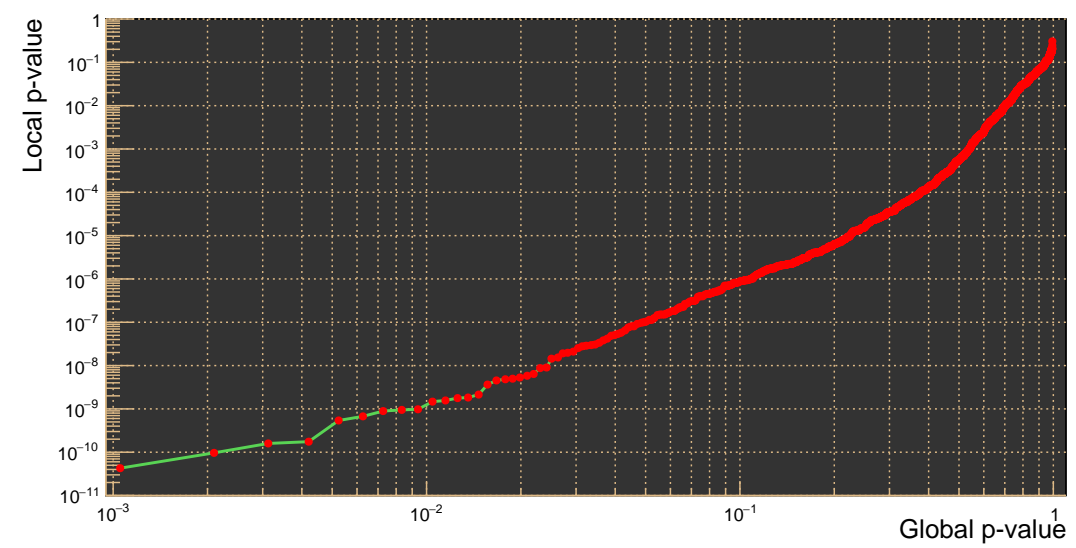

Figure 7.3.7.: The mapping between local p-values and global p-values for $\gamma^{\prime}$ masses above $0.023 \mathrm{GeV}$ for 949 toy-distributions generated from the model without signal. 


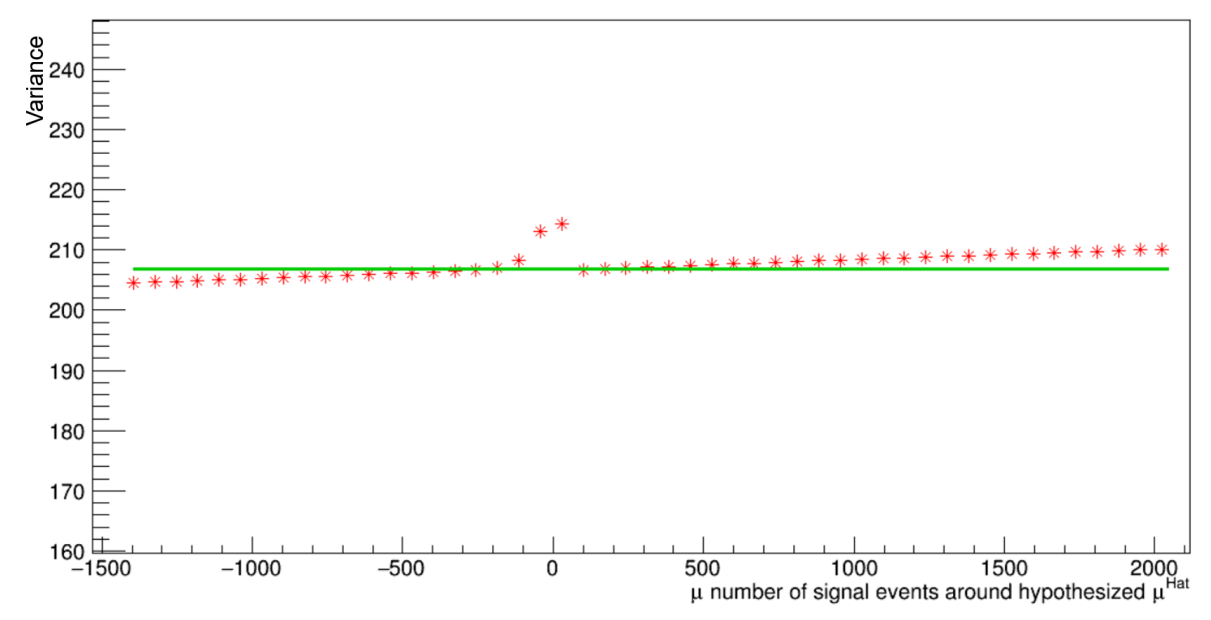

Figure 7.3.8.: The variance calculated from the inverse covariance matrix element (green line) and from eq.(7.3.19) for various $\mu$ values on the $X$-axis (red star points).

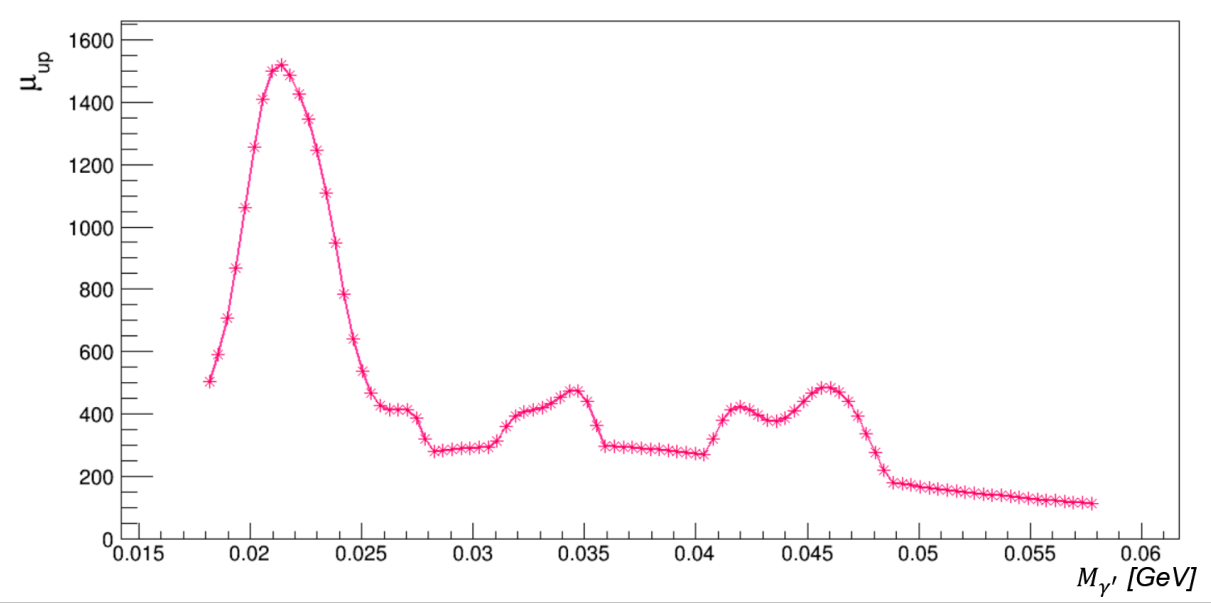

Figure 7.3.9.: The $\mu$ upper value from eq.(7.3.16) at the $95 \%$ confidence level. 


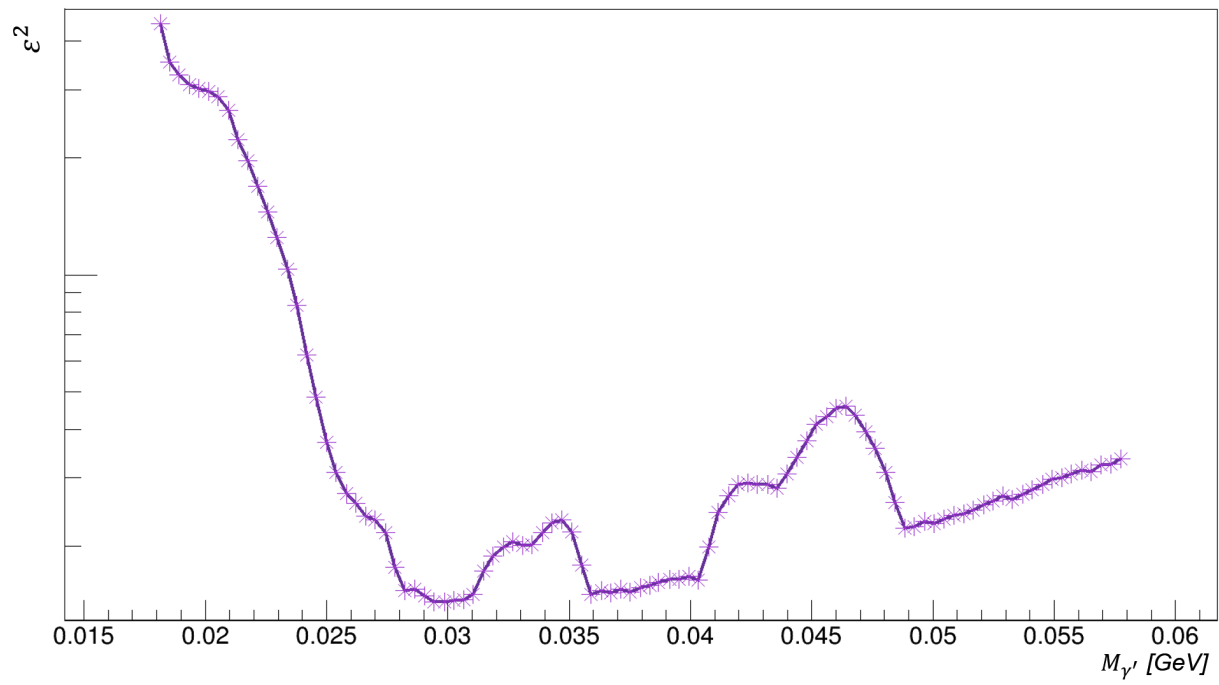

Figure 7.3.10.: The $\varepsilon^{2}$ coupling constant as a function of $M_{\gamma^{\prime}}$ at the $95 \%$ confidence level. The scale of the $Y$ - axis is hidden until the results are approved by the HPS collaboration. 


\section{Summary}

\section{"The whole visible world is perhaps nothing other than a motivation of man's wish to rest for a moment-an attempt to falsify the fact of knowledge, to try to turn the knowledge into the goal."}

Franz Kafka

The dark photon is conjectured in theories motivated from cosmology and particle physics. As a SM photon "reflection" in the hidden sector, the dark photon can interact very weakly with SM particles through mixing with ordinary photons. Such interactions are allowed by the kinetic mixing mechanism. Many hidden sector models contain particles suitable for dark matter candidates, motivating searches for dark photons.

The dark photon has raised an increased interest in experimental particle physics. We have reviewed the experiments searching for a $\gamma^{\prime}$ in different decay channels using various techniques. Several beam-dump experiment results conducted previously were revisited for dark photon search, and new such experiments are planned or ongoing. Fixed target and collider experiments are engaged in dark photon searches as well covering at small couplings for a wide range of masses. In addition to the resonance searches, displaced vertex search is available for some fixed target experiments. In particular, HPS is exploring both ways.

HPS is a fixed target experiment with a high-intensity beam impinging on a tungsten target at JLab. The detector consists of a SVT tracker and an ECal calorimeter to detect forward $e^{+} e^{-}$decays of the dark photon. The ECal provides a fast trigger signal necessary to manage the high background rates. The tracker measures the tracks and the energy of the particles. The properties of the SVT allow for close detection of the decay products, providing reliable data for the displaced vertex search as well as fine mass resolution for resonance searches.

The detector performance was discussed with a focus on the ECal corrections, including geometry correction, energy resolution and timing properties. The geometry simulation was updated in order to make it more flexible and to apply transformations obtained from position surveys of the detectors. The effect of the geometry correction on the cluster energy was found to be $\sim 1 \%$. The time resolution and the timing of the ECal have been studied at different energies, with cosmics and with full energy electrons. We have developed a cosmic track searching algorithm for these studies and found the time resolution to be $\sim 4 \mathrm{~ns}$. The resolution with the 
FEE events, with beam RF signal as a reference time, gave a resolution of $\sim 0.4 \mathrm{~ns}$ at low energy. The pile-up in the ECal was evaluated as well, showing only a few percent contamination, even at high beam currents.

The HPS signal has several irreducible QED background processes. Our resonance search requires to understand these backgrounds to evaluate the dark photon production ratio relative to all other processes. The QED backgrounds are studied with simulations using various event generators. The discrepancy between the simulations based on MadGraph4 and MadGraph5 motivated to study these generators with an independent theoretical calculation. To do so, we used a Monte-Carlo numerical integration-based theoretical framework developed by Beranek-Vanderhaeghen. A more accurate parametrization of the HPS acceptance was implemented in the code. However, the implementation of the acceptance for a large $\varphi_{\text {hor }}$ - range turned out to be problematic for the Monte Carlo integration. It was found also that the background in Ref.[125] was calculated for a positron beam instead of an electron beam. The comparison with the correct beam charge shows differences of the order of 20\%. The comparison of MG4 and MG5 with this calculation has shown a better agreement with MG5, confirming it as the main event generator for the HPS background simulations. There are still some differences between all 3 generators that are not understood. The comparison of different contributions points to the way the generators handle the antisymmetrization.

Moller scattering was analyzed to evaluate the mass resolution of the HPS detector at a given mass point. This study showed that the mass resolution is better when the vertexing algorithm is constrained to the target rather than unconstrained. The resolution at this mass point is, however, still larger than with the simulations.

The electron positron pairs coming from the dark photon decay were selected based on the kinematical properties of the dark photon production, in order to reduce the pairs coming from the background QED processes. From the initial sample, about $0.66 \%$ of the $e^{+} e^{-}$pairs survive after event selection. The comparison of the $0.5 \mathrm{~mm}$ and $1.5 \mathrm{~mm}$ data set don't show the same discrimination power for WAB events. To fully understand this feature and the WAB presence in the $1.5 \mathrm{~mm}$ data set, further studies with simulations will be necessary.

The invariant mass of the $e^{+} e^{-}$spectrum was then used for our statistical analysis, searching for a $\gamma^{\prime}$ resonance peak. The hypothesis test is performed comparing the data with a model without a resonance peak. The p-values indicate small fluctuations in the invariant mass spectrum. The studies of the model and the local p-values with pseudo-data sample-distributions, generated from the model with no signal, show a bias for these fluctuations at small masses in the left tail of the invariant mass distribution. However, it is unclear if this region with fast-varying acceptance can be recovered with a different parametrization. We also noted that the local to global p-value conversion might be biased by this problem.

We established a full analysis chain, using blinded data to extract the exclusion limits on the mass and kinetic mixing parameter of the dark photon at a $95 \%$ confidence 
level. The mass coverage of HPS with the data sample analyzed here is estimated to be from $18 \mathrm{MeV}$ to $58.7 \mathrm{MeV}$, which is smaller than the expectations from the HPS proposal. This is due to the acceptance region which is smaller than initially considered. 



\section{Résumé en Français}

Notre compréhension des interactions fondamentales et de l'évolution de l'univers ne peut décrire qu'une fraction du contenu de l'univers. La matière noire et l'énergie sombre ont été introduites pour combler cette lacune, pourtant elles restent insaisissables dans nos recherches de détection directe. L'idée du photon sombre a aussi des motivations indépendantes en physique des particules théoriques et en cosmologie, où de nouvelles symétries ont été introduites pour résoudre certaines questions ouvertes du modèle standard. Celles-ci impliquent souvent un secteur caché avec ses états de matière cachés et ses interactions. Le photon sombre est l'un des ponts possibles pour relier la matière dans le monde visible à la matière dans le secteur caché. De nombreux candidats à la matière noire se trouvent dans ce type de secteurs cachés.

Le photon sombre est conjecturé, en tant que "réflexion" du photon standard dans le secteur caché, le photon sombre peut interagir très faiblement avec les particules du modèle standard par l'intermédiaire des photons ordinaires. De telles interactions sont permises par le mécanisme de mélange cinétique. De nombreux modèles de secteurs cachés contiennent des particules candidates pour la matière noire, ce qui constitue la principale motivation pour les recherches de photons sombres.

Le photon sombre a suscité récemment un intérêt particulier en physique des particules. Nous avons passé en revue les expériences recherchant un photon sombre dans différents canaux de désintégration et utilisant diverses techniques. Plusieurs résultats d'expériences sur bloc d'arrêt de faisceau effectués dans le passé ont ainsi été revisitée pour la recherche de photons sombres et de nouvelles expériences de ce type sont même planifiées ou en cours. Des expériences sur cible fixe et en collisionneur sont aussi en cours pour des recherches de photons sombres couvrant de petits couplages et une large gamme de masses. En plus des recherches par résonance, la recherche de vertex déplacée est possible pour certaines expériences sur cibles fixes. En particulier, HPS (Heavy Photon Search) à JLab explore ces deux voies.

Le travail présenté dans cette thèse est consacrée à la recherche d'un photon sombre dans l'expérience HPS à JLab. JLab est un accélérateur avec un faisceau d'électrons continu de 1 a $12 \mathrm{GeV}$. On s'attend à ce que les photons sombres soient produits par un processus semblable au Bremsstrahlung lorsque le faisceau d'électrons de haute intensité interagit avec une cible de tungstène. La production de photons sombres, suivie de leurs désintégrations en paires électron-positron, est un événement rare qui doit être détecté dans une quantité abondante de bruit de fond QED. Le détecteur HPS est équipé d'un calorimètre électromagnétique et d'un trajectographe de vertex 
en silicium comme illustré dans la Fig. 7.4.1. HPS a eu une prise de donné d'essai réussie en 2012 et a pris des premières données pour la physique sur la période 20142016. Le travail présenté dans cette thèse est axée sur l'analyse en aveugle d'une fraction des données de 2015 , où le trajectographe silicium était placé à $1.5 \mathrm{~mm}$ de la ligne de faisceau.

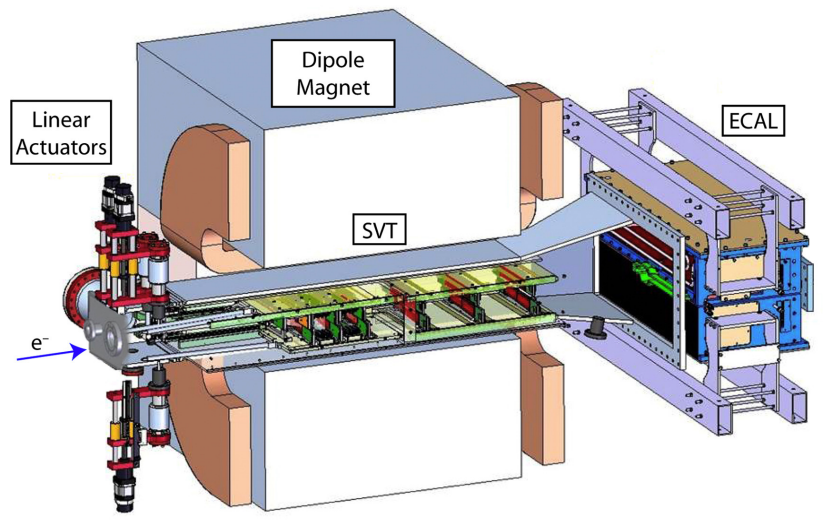

Figure 7.4.1.: L'aimant et les détecteurs de HPS.

Les performances du détecteur ont été discutées en mettant l'accent sur les corrections apportées aux données du calorimètre, en particulier la correction de sa géométrie, la résolution de l'énergie et ses propriétés temporelles. La simulation de la géométrie du détecteur a été mise à jour afin de la rendre plus flexible et d'appliquer les transformations obtenues à partir des relevés de position des détecteurs. L'effet de la correction de la géométrie sur l'énergie a été éstimé à $\sim 1 \%$. La résolution temporelle du calorimètre ont été étudiés à différentes énergies, avec des rayons cosmiques et avec des électrons de haute énergie. Nous avons développé un algorithme de recherche de trajectoire pour l'étude des rayons cosmiques et trouvé que la résolution temporelle était d'environ $4 \mathrm{~ns}$. La résolution pour les événements de haute énergie et en utilisant le signal RF du faisceau comme temps de référence, atteint $\sim 0.4 \mathrm{~ns}$. L'empilement d'évènements dans l'ECal a également été évalué, dans la Fig. 7.4.2 on peut voir qu'elle ne représente que quelques pourcents de contamination, même à des courants de faisceau élevés.

Le signal de l'expérience reçoit du bruit de fond de plusieurs processus QED, qui sont irréductibles. Notre recherche de résonance nécessite de comprendre ces bruits de fond pour évaluer le rapport entre la production de photons sombres et tous les autres processus. Le bruit de fond QED est étudié avec des simulations en utilisant divers générateurs d'événements. L'écart entre les simulations basées sur MadGraph4 et MadGraph5 pour le calcul des diagrammes présentés dans la Fig.7.4.3 a motivé la comparaison de ces générateurs avec un calcul théorique indépendant. Pour ce faire, nous avons utilisé un cadre théorique basé sur l'intégration numérique MonteCarlo développé par Beranek-Vanderhaeghen. Une paramétrisation plus précise de l'acceptance de HPS a été implémentée dans le code. Cependant, la mise en oeuvre 

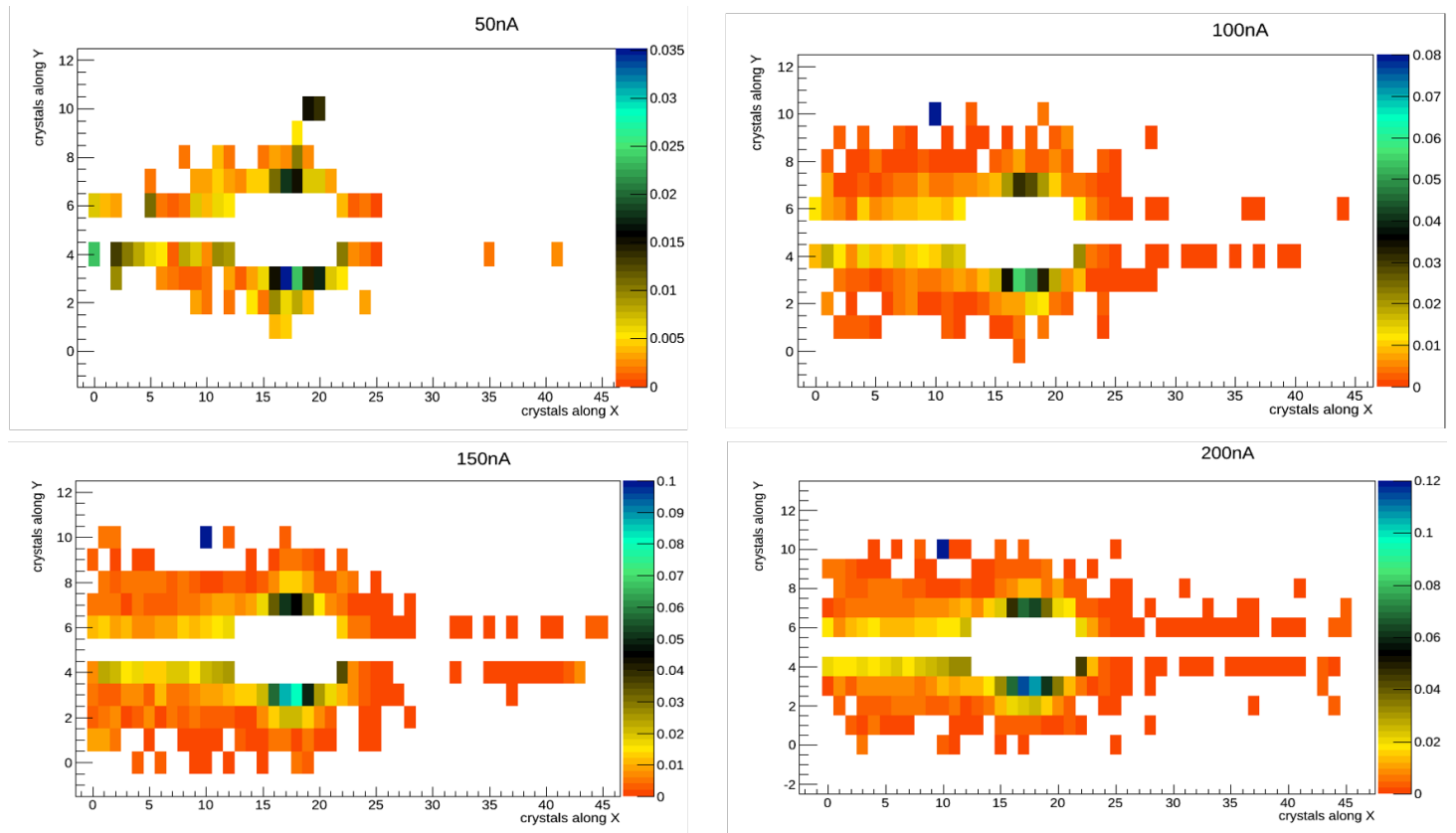

Figure 7.4.2.: Effet d'empilement dans le calorimètre, présenté comme un rapport du nombre de signaux avec deux pics sur ceux à un pic avec un courant de faisceau de $50 \mathrm{nA}, 100 \mathrm{nA}, 150 \mathrm{nA}$, et $200 \mathrm{nA}$. Chaque coordonnée (x, y) correspond à un cristal du calorimètre, la palette de couleur de l'axe $Z$ est le rapport d'empilement.

de l'acceptance pour un grand espace de phase s'est avérée problématique pour l'intégration Monte Carlo. Il a également été constaté que le calcule original des auteurs était pour un faisceau de positons au lieu d'un faisceau d'électrons. La comparaison avec la charge de faisceau correcte montre des différences de l'ordre de 20\%. La comparaison de MadGraph4 et MadGraph5 avec ce calcul a montré un meilleur accord avec MadGraph5, le confirmant comme le générateur d'événement principal pour les simulations de bruit de fond pour HPS. Il y a encore quelques différences entre les trois générateurs qui ne sont pas comprises. La comparaison des différentes contributions indique que la source du problème pourrait être la manière dont les générateurs gèrent l'antisymétrisation.

La diffusion de Moller a été analysée pour évaluer la résolution en masse du détecteur HPS à un point en masse donné. Cette étude a montré que la résolution en masse est meilleure lorsque l'algorithme de reconstruction des traces est contraint par un point d'origine à la cible plutôt que non contrainte. La résolution à ce point de masse, présenté dans la Fig. 7.4.4, est cependant plus grande que l'estimation des simulations.

Ensuite, les paires positon-électron ont été sélectionnées sur la base de leurs propriétés cinématiques pour maximiser la sélection de photons sombres et afin de réduire les paires provenant des processus QED. À partir de l'échantillon initial, 


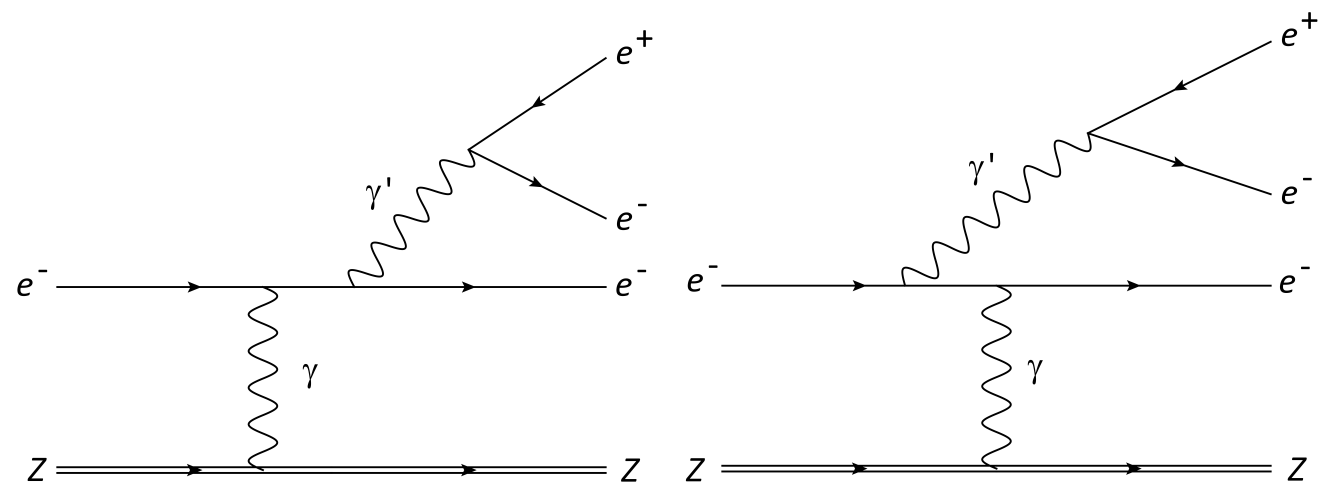

Figure 7.4.3.: Les diagrammes de Feynman des processus recherchés dans l'expérience HPS.

environ $0,66 \%$ des paires survivent après la sélection des événements. La comparaison des jeux de données à $0.5 \mathrm{~mm}$ et $1.5 \mathrm{~mm}$ n'indique pas la même puissance de discrimination pour certains événements du bruit de fond. Pour mieux comprendre cette différence et les contributions de QED à l'ensemble de données de $1.5 \mathrm{~mm}$, d'autres études et simulations seront nécessaires.

La masse invariante du spectre $e^{-} e^{+}$a ensuite été utilisée pour une analyse statistique où nous cherchions un pic de résonance. Le test d'hypothèse est effectué en comparant les données avec un modèle sans pic de résonance. Les valeurs-p obtenues en comparant ces deux analyses statistiques sont présentées dans la Fig. 7.4.5 et indiquent de petites fluctuations dans le spectre de masse invariant. Le modèle et la distribution des valeurs-p ont été étudiés en créant des échantillons de pseudodonnées, générées à partir du modèle sans signal. Celles-ci montrent un biais dans les fluctuations de valeurs-p à petites masses. Il n'est pas clair si cette région, avec une acceptance variant rapidement, pourra être récupérée avec une paramétrisation différente. Nous avons également noté que la conversion de la valeur-p locale à globale pouvait être biaisée par ce problème.

Nous avons établi une chaîne d'analyse complète, en utilisant $10 \%$ des données disponibles pour extraire les limites d'exclusion sur le paramètre de mélange de masse et de couplage du photon sombre à un niveau de confiance de 95\%. La couverture en masse de HPS avec l'échantillon de données analysé est présentée dans la Fig. 7.4.6. On observe une sensibilité de l'expérience entre $18 \mathrm{MeV}$ et $58.7 \mathrm{MeV}$, ce qui est inférieur aux attentes initiales. Cette différence est due à l'acceptance à petit angle qui est plus petite que celle initialement envisagée. 

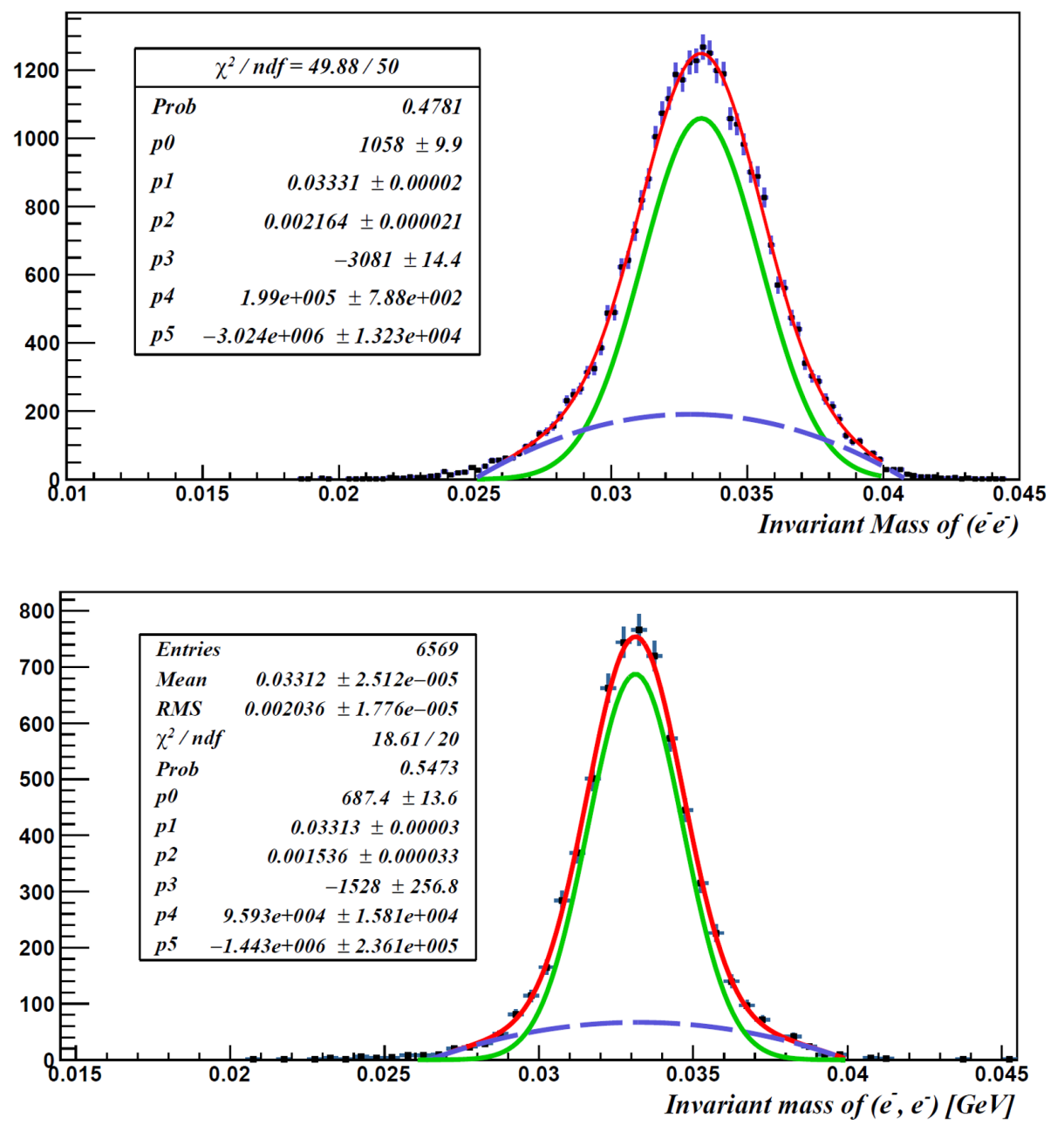

Figure 7.4.4.: Distribution de la masse invariante des paires d'électrons de l'échantillon de données avec le SVT à $1.5 \mathrm{~mm}$, un vertex non contraint en haut, et avec un vertex contraint en bas. La ligne rouge est un ajustement utilisant une fonction composite, formée d'une gaussienne (trait plein vert) et d'un polynôme du second ordre (trait pointillé violet). 


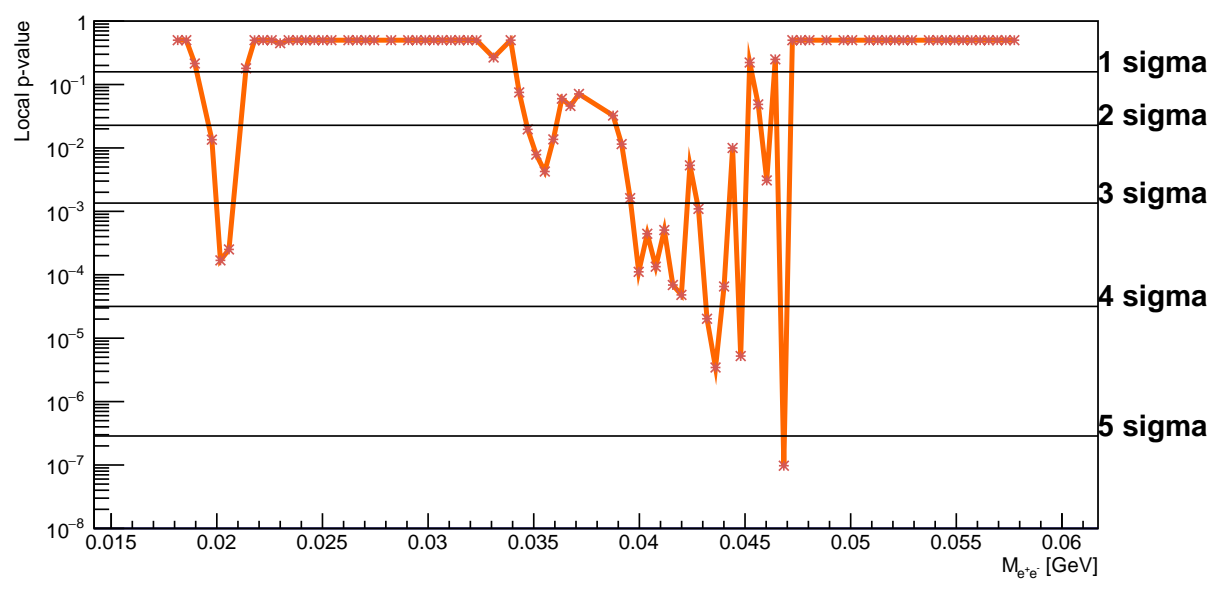

Figure 7.4.5.: La valeur-p locale en fonction de la masse hypothétique du $\gamma^{\prime}$ obtenue en utilisant un échantillon de données.

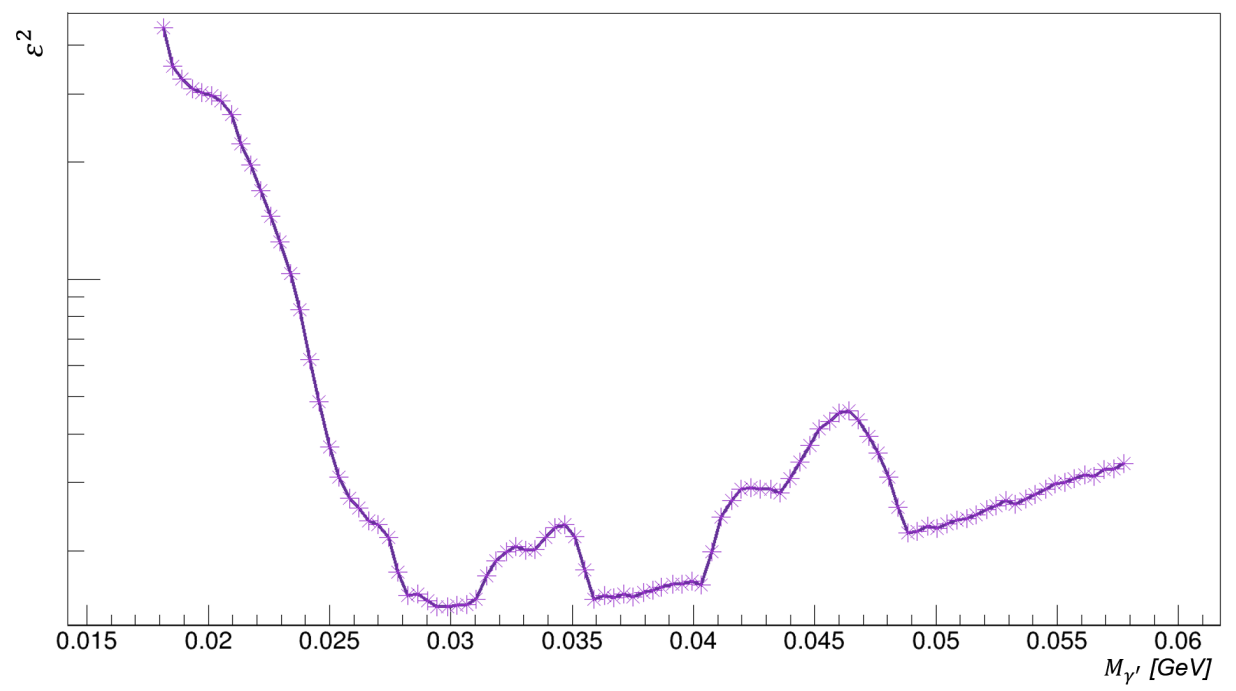

Figure 7.4.6.: La constante de couplage $\varepsilon^{2}$ en fonction de $M_{\gamma^{\prime}}$ a un niveau de confiance de $95 \%$. L'échelle de l'axe $Y$ est masquée jusqu'à ce que les résultats soient approuvés par la collaboration HPS. 


\section{A. Acceptance Parametrization of the ECal}

The fiducial region used in the background simulation is evaluated here from the calorimeter acceptance coverage. We simulated electrons and positrons with energies uniformly distributed between 0 and $2 \mathrm{GeV}$ are sent towards the calorimeter in directions such that the azimuthal angle is in the range $0<\varphi<\pi$ and the polar angle is in the range $0<\cos \theta<1$. For this work, we use the ECal detector simulation implemented in SLIC and the output is processed through the standard HPS readout simulation-reconstruction chain.

\section{A.1. Coordinate Systems}

\section{The coordinate system used for the acceptance study}

First, we define the coordinate system and the angular framework used to give the acceptance limits. The coordinate system is defined so that it is particularly convenient for the geometry of the HPS acceptance. This coordinate system is similar to the one used in the Beranek et al. article [125]. In the next section, the differences are detailed. The horizontal angle $\varphi_{\text {hor }}$ is the angle between the projection of the momentum vector of the scattered particle and the $Z$-axis and $\theta_{\text {out }}$, referred to as the out-of-plane angle, is the angle between the particle's momentum and the $X-Z$ plane. These angles are illustrated in Fig. A.1.1. The conversion to the Cartesian coordinate system can be given as:

$$
\begin{aligned}
& x=|\vec{p}| \cos \theta_{\text {out }} \sin \varphi_{\text {hor }} \\
& y=|\vec{p}| \cos \theta_{\text {out }} \\
& z=|\vec{p}| \cos \theta_{\text {out }} \cos \varphi_{\text {hor }}
\end{aligned}
$$

\section{The coordinate system used in Ref.[125] for HPS}

In the article of Ref.[125], the authors have calculated the expected QED background cross-section for HPS within an acceptance defined by the horizontal angle and the out-of-plane angle. While the horizontal angle is the same as in the left panel of 


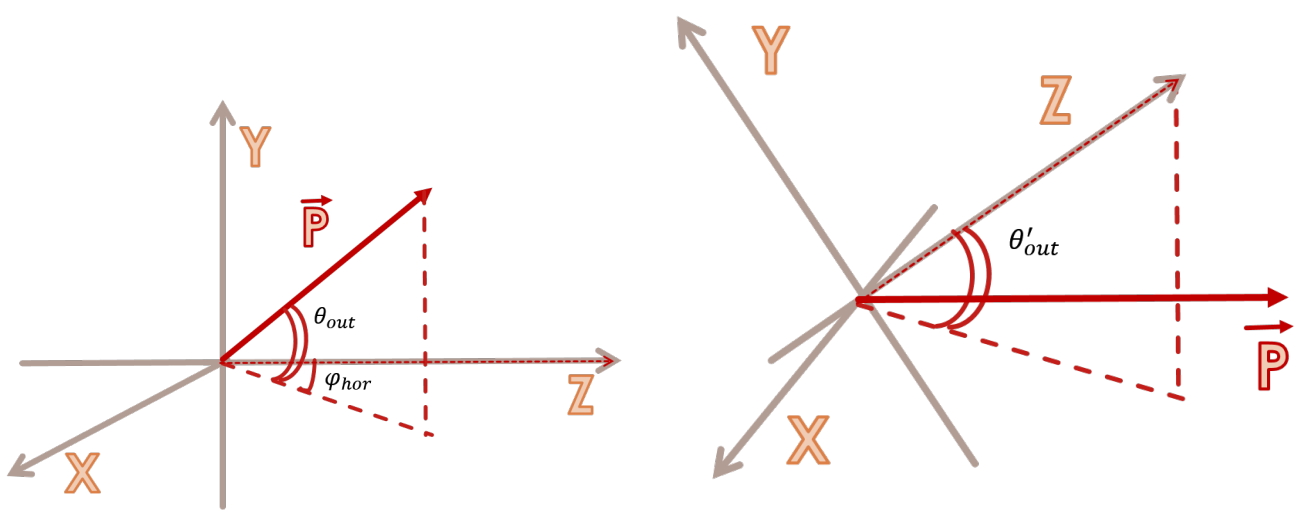

Figure A.1.1.: The coordinate system used for the acceptance calculations in this study (left). The definition of the out-of-plane angle used in Ref.[125] for HPS (right).

Fig. A.1.1, the out-of-plane angle, $\theta_{\text {out }}^{\prime}$, is the angle between the $Z$-axis and the plane formed by the $X$-axis and the particle momentum $\vec{p}$, as depicted in Fig. A.1.1 (right). The relation with the Cartesian system is

$$
\begin{aligned}
& x^{2}=\frac{\tan ^{2} \varphi_{\text {hor }}}{\tan ^{2} \varphi_{\text {hor }}+\tan ^{2} \theta_{\text {out }}^{\prime}+1}, \\
& y^{2}=\frac{\tan ^{2} \theta_{\text {out }}^{\prime}}{\tan ^{2} \varphi_{\text {hor }}+\tan ^{2} \theta_{\text {out }}^{\prime}+1}, \\
& z^{2}=\frac{1}{\tan ^{2} \varphi_{\text {hor }}+\tan ^{2} \theta_{\text {out }}^{\prime}+1}, \\
& \operatorname{sgn}(x)=\operatorname{sgn}\left(\varphi_{\text {hor }}\right) \\
& \operatorname{sgn}(y)=\operatorname{sgn}\left(\theta_{\text {out }}^{\prime}\right)
\end{aligned}
$$

While the definition is different, the differences in the numerical values of $\theta_{\text {out }}^{\prime}$ and $\theta_{\text {out }}$ are negligibly small in the HPS acceptance. In the following, we use the $\theta_{\text {out }}$ definition of the out-of-plane angle for the acceptance study explained in the previous section, and directly apply it for the background simulation in chapter 5 , without converting $\theta_{\text {out }}$ into $\theta_{\text {out }}^{\prime}$.

\section{A.2. Parametrization of The ECal Acceptance}

\section{Excluding the beam cage}

The acceptance study is carried out for electrons and repeated for positrons in a similar way. The figures presented here are for the studies with electrons. However, the final results are summarized in a figure including the acceptance calculation 
for positrons. Because of the dipole field, the out-of-plane angle is not dependent from the particle energy, while the horizontal angle is. To calculate this energydependent acceptance limit, we use simulated electrons and positrons of different energies relevant to HPS.
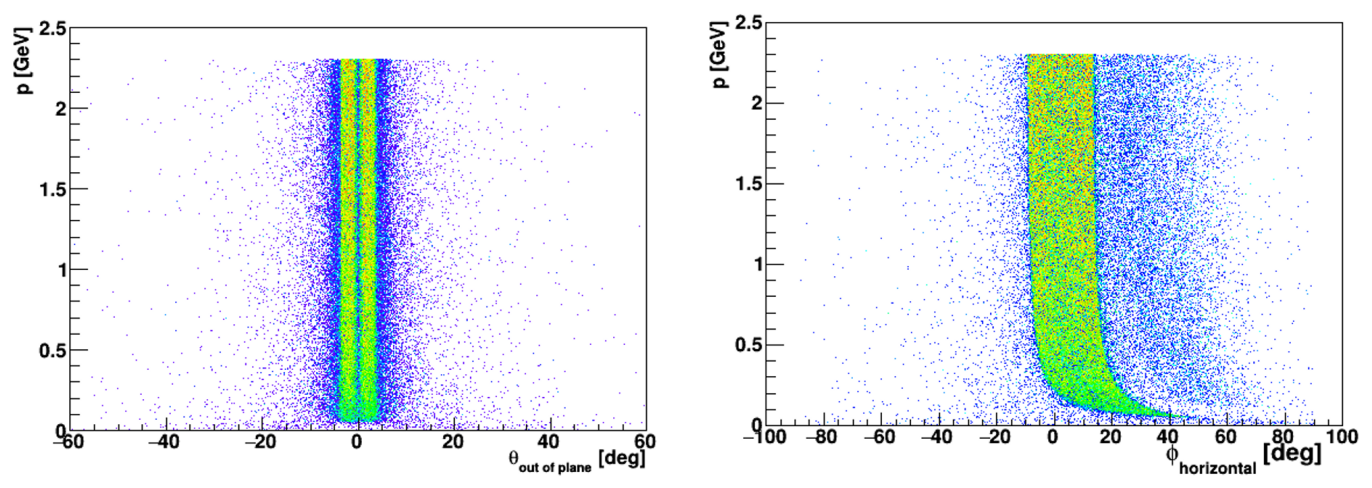

Figure A.2.1.: On the left is the out-of-plane angle vs energy distribution, and on the right is the horizontal angle vs energy distribution for electrons.

From all events registered in the ECal after the readout simulation and reconstruction, we select only the events where the parent particles are the simulated electrons (positrons). The distributions of the out-of-plane angle and of the horizontal angle against the particle energy are constructed, as shown in Fig. A.2.1 and analyzed. As shown in Fig. A.2.2, the fiducial region is divided in three zones, in order to separate the region around the beam where there are less crystals than on the sides. The limits for the horizontal and the out-of-plane angle in each of the zones, 1, 2 and 3, are calculated independently.
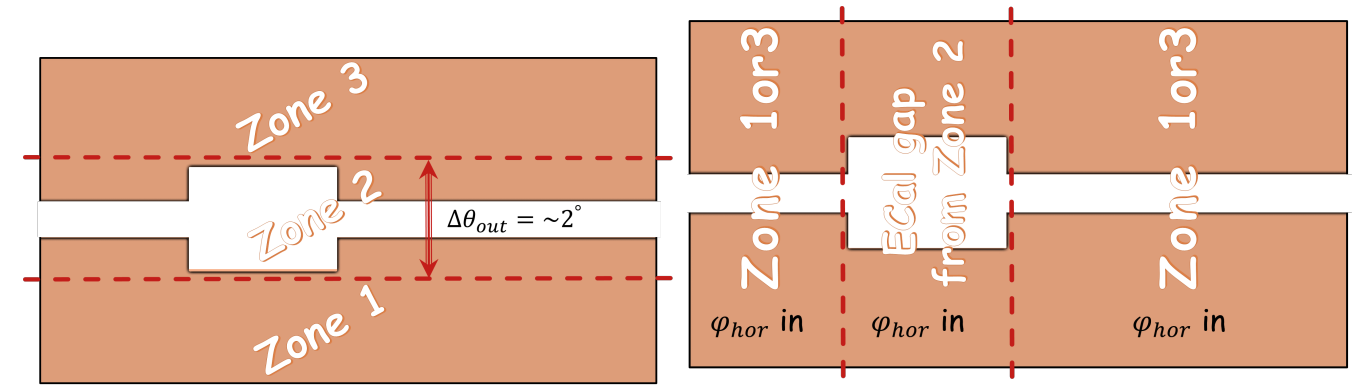

Figure A.2.2.: The ECal schematic view showing the division in zones, in order to parametrize the acceptance taking into account the ECal gap. On the left, the ECal is divided in 3 zones based on $\theta_{\text {out }}$, in order to study the $\varphi_{\text {hor }}$ limits. On the right, we show the ECal zones, when $\varphi_{\text {hor }}$ is in the 1,2 or $3 \theta_{\text {out }}-$ zone, in order to study the $\theta_{\text {out }}$ limits in the ECal gap.

The low and upper limits for the horizontal angle are found from an ad-hoc function search, selecting the green region on the left plot of Fig. A.2.1. To extract the 
exclusion limits for the ECal gap around the beam, the inner edges of the horizontal angle distribution are fitted in energy bins of size $\sim 0.1 \mathrm{GeV}$. Such a slice is shown in the right plot of Fig. A.2.3. The inner parts of the distribution are fitted with a line, and the values at $75 \%$ from the maxima are selected for each slice. These values are then fitted to extract the limits outlining the gap in the zone 2 , shown on the left plot in Fig. A.2.3. The fiducial limits extracted from this calculation for $\varphi_{\text {hor }}$ are illustrated in Fig. A.2.4 as red solid lines. The light blue lines in both plots indicate the limits used in Ref.[125].

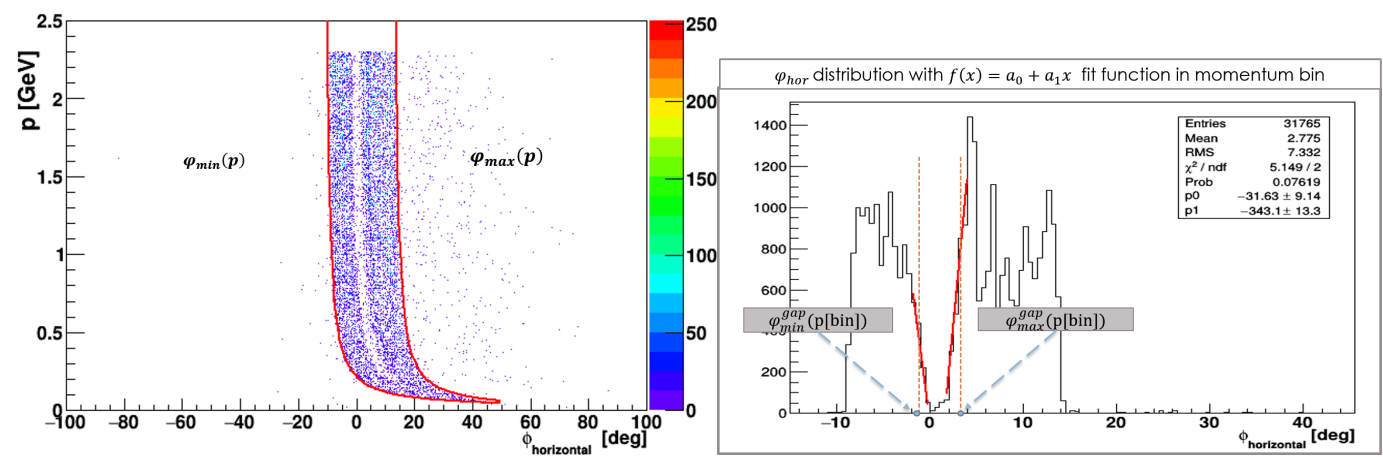

Figure A.2.3.: On the left is the horizontal angle vs energy distribution and on the right is the horizontal angle distribution in a given energy bin. The inner edges are fitted (red solid lines) and the values at $\sim 75 \%$ (dashed red lines) of the fit maximum are extracted.
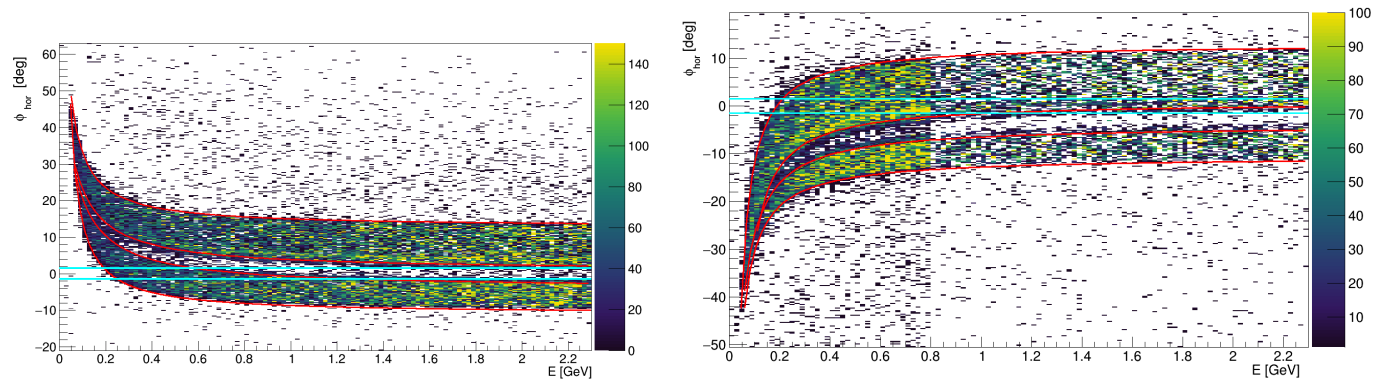

Figure A.2.4.: The horizontal angle distribution vs the particle energy in the zone 2. The red lines correspond to the acceptance limits for the horizontal angle for electrons (left) and positrons (right). The high statistics region visible for the positrons show the excess of positrons generated to have a better fitting resolution at low energies. The bright blue lines show the acceptance used for the horizontal angle in Ref.[125].

To extract the out-of-plane angle limits excluding the ECal gap, the energy dependent distribution of $\theta_{\text {out }}$ is studied for each $\varphi_{\text {hor }}$ in zone1, ECal gap in zone 2 and zone 3, as illustrated in Fig. A.2.2 (right). The out-of-plane angle limits for each region of $\varphi_{\text {hor }}$ is set, based on constant cuts, as shown in Fig. A.2.5. 


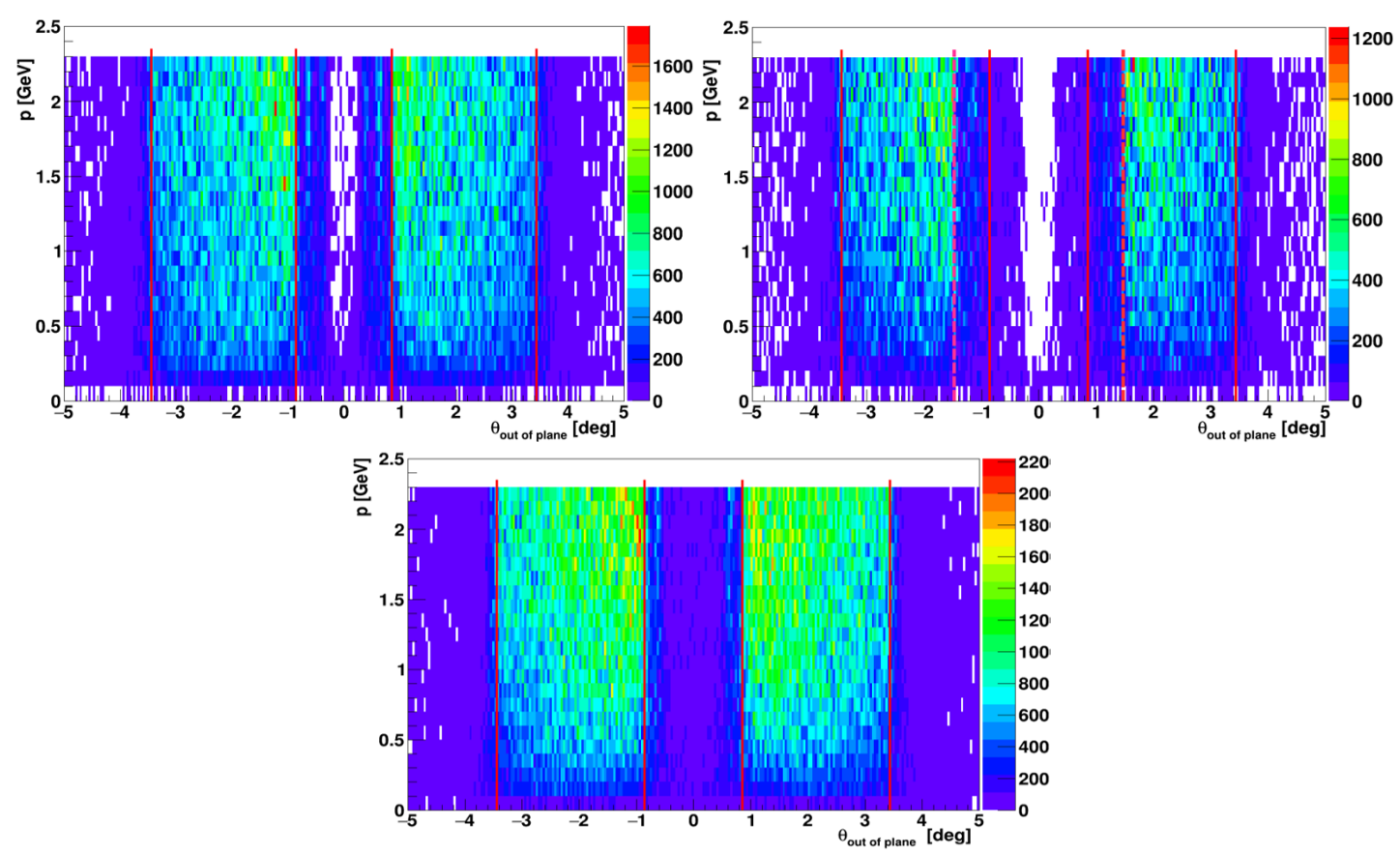

Figure A.2.5.: The out-of-plane angle distribution vs energy for electrons. The red lines represent the fiducial limits for each zone, when $\varphi_{\text {hor }}$ is in zone 1 (top left), 2 (top right) and 3 (bottom). The dashed lines for zone 2 corresponds to the acceptance limits for this region. The outer acceptance limit is indicated with solid lines in zone 2. The inner solid lines correspond to the inner acceptance limits for zone 1 and 3 . When compared with the dashed lines, it shows the impact of the ECal gap on the acceptance range in zone 2 .

\section{A.2.1. Results}

\section{Fiducial Region for the Horizontal Angle}

The functions for the fiducial region for electrons are given below ${ }^{1}$ :

- Lower limit - $\varphi_{\min }(p[G e V])[d e g]=-11+\frac{1}{e(p-0.009)}$

- Upper limit - $\varphi_{\max }(p[G e V])[d e g]=12.75+\frac{1}{e(p+0.013)}$

- Lower gap limit - $\varphi_{\min }^{\text {gap }}(p[\mathrm{GeV}])[\mathrm{deg}]=-4.098+\frac{3.203}{p+0.0446}$

- Upper gap limit - $\varphi_{\text {max }}^{\text {gap }}(p[\mathrm{GeV}])[\mathrm{deg}]=0.806515+\frac{3.20542}{p+0.04405}$

The results for the positrons are:

- Lower limit - $\varphi_{\min }(p[G e V])[d e g]=-10.5-e p^{-0.94}$

- Upper limit - $\varphi_{\max }(p[G e V])[d e g]=1.16-\frac{2.83162}{p+0.01128}$

- Lower gap limit - $\varphi_{\min }^{\text {gap }}(p[\mathrm{GeV}])[\mathrm{deg}]=-3.9709-\frac{2.5817}{p+0.01296}$

\footnotetext{
${ }^{1} e$ is the Euler's number: $e=2.71828$.
} 
- Upper gap limit - $\varphi_{\text {max }}^{\text {gap }}(p[G e V])[\operatorname{deg}]=1.1675-\frac{2.8316}{p+0.01127}$

Fiducial Region for the out-of-plane Angle

The limits of the out-of-plane angle $\theta_{\text {out }}$ for electrons and positrons are:

- Zone 1 and Zone 3 - $\theta_{\text {out }}[\mathrm{mrad}] \in[-50 ;-15] \cup[15 ; 50]$

- Zone 2 - $\theta_{\text {out }}[\mathrm{mrad}] \in[-50 ;-25.44] \cup[25.44 ; 50]$ 


\section{Bibliography}

[1] J. L. Feng et al. Planning the Future of U.S. Particle Physics (Snowmass 2013): Chapter 4: Cosmic Frontier. 2014. 4

[2] M. A. Deliyergiyev. Recent Progress in Search for Dark Sector Signatures. Open Phys., 14(1):281-303, 2016. 5

[3] H. Poincaré. Poincaré - Oeuvres (20). lci-eBooks, 2016. 5

[4] J. H. Oort. The force exerted by the stellar system in the direction perpendicular to the galactic plane and some related problems. Bulletin of the Astronomical Institutes of the Netherlands , 6:249, August 1932. 5

[5] F. Zwicky. Republication of: The redshift of extragalactic nebulae. General Relativity and Gravitation, 41:207-224, January 2009. 5

[6] V. C. Rubin, W. K. Ford Jr. Rotation of the Andromeda Nebula from a Spectroscopic Survey of Emission Regions. Astrophys. J., 159:379-403, 1970. 6

[7] E. P. Verlinde. Emergent Gravity and the Dark Universe. SciPost Phys. 2 (2017) no.3, 016, 2016. no. 3, Nov 7, 2016, p. 1-51 . 6

[8] M. Margot Brouwer et al. First test of Verlinde's theory of Emergent Gravity using Weak Gravitational Lensing measurements. Mon. Not. Roy. Astron. Soc., 466(3):2547-2559, 2017. 6

[9] R. H. Sanders K. G. Begeman, A. H. Broeils. Extended rotation curves of spiral galaxies: Dark haloes and modified dynamics. Mon. Not. Roy. Astron. Soc., 249:523, 1991. 7

[10] F. Zwicky. On the Masses of Nebulae and of Clusters of Nebulae. Astrophysical Journal, 86:217, 1937. Provided by the SAO/NASA Astrophysics Data System http://adsabs.harvard.edu/abs/1937ApJ...86..217Z . 6

[11] Sir I. Newton. Opticks: or, A treatise of the reflections, refractions, inflexions and colours of light. Also two treatises of the species and magnitude of curvilinear figures. 1704. 6

[12] F. W. Dyson, A. S. Eddington, C. Davidson. A Determination of the Deflection of Light by the Sun's Gravitational Field, from Observations Made at the Total Eclipse of May 29, 1919. Philosophical Transactions of the Royal Society of London A: Mathematical, Physical and Engineering Sciences, 220(571581):291-333, 1920. 7 
[13] F. Walsh, R. F. Carswell, R. J. Weymann. 0957 + 561 A, B - Twin quasistellar objects or gravitational lens. 279:381-384, 1979. 7

[14] T. J. Broadhurst N. Benitez E. van Kampen A. N. Taylor, S. Dye. Gravitational lens magnification and the mass of abell 1689. Astrophys. J., 501:539, 1998. 7

[15] M. Markevitch et al. : X-ray NASA/CXC/CfA/; D. Clowe et al. : Lensing Map: NASA/STScI; ESO WFI;Magellan/U.Arizona/ D. Clowe et al. : Optical : NASA/STScI; Magellan/U.Arizona/. The Matter of the Bullet Cluster, 2006. 8

[16] M. Markevitch. Chandra observation of the most interesting cluster in the universe. 2005. [ESA Spec. Publ.604,723(2006)]. 8

[17] A. H. Gonzalez M. Markevitch S. W. Randall C. Jones et al. D. Clowe, M. Bradac. A direct empirical proof of the existence of dark matter. Astrophys. J., 648:L109-L113, 2006. 8

[18] G.W. Angus, B. Famaey, and H. Zhao. Can MOND take a bullet? Analytical comparisons of three versions of MOND beyond spherical symmetry. Mon. Not. Roy. Astron. Soc., 371:138, 2006. 8

[19] B. Famaey, S. McGaugh . Modified Newtonian Dynamics (MOND): Observational Phenomenology and Relativistic Extensions. Living Rev. Rel., 15:10, 2012. 8

[20] D. N. Schramm. The Big Bang and Other Explosions in Nuclear and Particle Astrophysics. World Scientific, 1996. 9

[21] R. A. Alpher, R. C. Herman. Remarks on the Evolution of the Expanding Universe. Physical Review, 75:1089-1095, 1949. 9

[22] R. H. Dicke, P. J. E. Peebles, P. G. Roll, D. T. Wilkinson. Cosmic Black-Body Radiation. Astrophysical Journal, 142:414-419, 1965. 9

[23] A. H. Guth. Inflationary universe: A possible solution to the horizon and flatness problems. Phys. Rev. D, 23:347-356, 1981. 10

[24] A. L. Coil. The Large-Scale Structure of the Universe. 2013. 11

[25] P. A. R. Ade et al. Planck 2015 results. XIII. Cosmological parameters. Astron. Astrophys., 594:A13, 2016. 13

[26] M. Betoule et al. . Improved cosmological constraints from a joint analysis of the SDSS-II and SNLS supernova samples. Astron. Astrophys., 568:A22, 2014. 14

[27] W. Scott, A. E. Lepsch. Content of the Universe Pie Chart, 2016. NASA Goddard Space Flight Center. 15

[28] Wikipedia. Standard Model. Wikipedia, The Free Encyclopedia, 2017. 16 
[29] G. Aad et al. Observation of a new particle in the search for the Standard Model Higgs boson with the ATLAS detector at the LHC. Phys. Lett., B716:129, 2012. 13

[30] S. Chatrchyan et al. Observation of a new boson at a mass of $125 \mathrm{GeV}$ with the CMS experiment at the LHC. Phys. Lett., B716:30-61, 2012. 13

[31] S. Dimopoulos AN. rkani Hamed. Supersymmetric unification without low energy supersymmetry and signatures for fine-tuning at the LHC. JHEP, 06:073, 2005. 15

[32] R. D. Peccei. The Strong CP problem and axions. Lect. Notes Phys., 741:3-17, 2008. [,3(2006)]. 15, 20

[33] H. R. Quinn R. D. Peccei. CP Conservation in the Presence of Instantons. Phys. Rev. Lett., 38:1440-1443, 1977. 15

[34] A. D. Sakharov. Violation of CP Invariance, c Asymmetry, and Baryon Asymmetry of the Universe. Pisma Zh. Eksp. Teor. Fiz., 5:32-35, 1967. [Usp. Fiz. Nauk161,61(1991)]. 16

[35] L. Yang A. Kusenko, L. Pearce. Postinflationary Higgs relaxation and the origin of matter-antimatter asymmetry. Phys. Rev. Lett., 114(6):061302, 2015. 16

[36] M. E. Shaposhnikov V. A. Rubakov. Electroweak baryon number nonconservation in the early universe and in high-energy collisions. Usp. Fiz. Nauk, 166:493-537, 1996. [Phys. Usp.39,461(1996)]. 16

[37] L. B. Okun. Space-time and vacuum as seen from Moscow. Int. J. Mod. Phys., A17S1:105-118, 2002. 16

[38] M. Shaposhnikov L. Canetti, M. Drewes. Matter and Antimatter in the Universe. New J. Phys., 14:095012, 2012. 16

[39] A. F. Pikelner O. L. Veretin A. V. Bednyakov, B. A. Kniehl. Stability of the electroweak vacuum: Gauge independence and advanced precision. Phys. Rev. Lett., 115:201802, 2015. 17

[40] E. Morgante A. Riotto L. Senatore et al. J. R. Espinosa, G. F. Giudice. The cosmological Higgstory of the vacuum instability. JHEP, 09:174, 2015. 17

[41] Ch. Yang T. D. Lee. Question of Parity Conservation in Weak Interactions. Phys. Rev., 104:254-258, 1956. 18

[42] I. Yu. Kobzarev and L. B. Okun, I. Ya. Pomeranchuk. On the possibility of experimental observation of mirror particles. Sov. J. Nucl. Phys., 3(6):837841, 1966. Yad. Fiz.3,1154(1966). 18

[43] L. B. Okun. Mirror particles and mirror matter: 50 years of speculations and search. Phys. Usp., 50:380-389, 2007. 18

[44] L. B. Okun. Muonic charge and muonic photons. Yadern. Fiz., 10: 35862(Aug. 1969)., Jan 1969. 18 
[45] C. N. Yang T. D. Lee. Conservation of heavy particles and generalized gauge transformations. Phys. Rev., 98:1501-1501, 1955. 18

[46] L. B. Okun. Limits of electrodynamics: paraphotons? Sov. Phys. JETP, 56:502, 1982. [Zh. Eksp. Teor. Fiz.83,892(1982)]. 18, 19

[47] B. Holdom. Two U(1)'s and Epsilon Charge Shifts. Phys. Lett., 166B:196-198, 1986. 18, 22, 24

[48] Z. Berezhiani. Through the looking-glass: Alice's adventures in mirror world. pages 2147-2195, 2005. 18

[49] G. G. Raffelt P. D. Serpico. MeV-mass dark matter and primordial nucleosynthesis. Phys. Rev., D70:043526, 2004. 18

[50] L. V. Laperashvili Ch. R. Das. Dark matter, mirror world, and e6 unification. Physics of Atomic Nuclei, 72(2):377-384, 2009. 19

[51] R. R. Volkas S. J. Lonsdale. Grand unified hidden-sector dark matter. Phys. Rev., D90(8):083501, 2014. [Erratum: Phys. Rev.D91,no.12,129906(2015)]. 19

[52] H. B. Nielsen A. Tureanu C. R. Das, L. V. Laperashvili. Mirror World and Superstring-Inspired Hidden Sector of the Universe, Dark Matter and Dark Energy. Phys. Rev., D84:063510, 2011. 19

[53] S. Alekhin et al. A facility to Search for Hidden Particles at the CERN SPS: the SHiP physics case. Rept. Prog. Phys., 79(12):124201, 2016. 19

[54] I. M. Shoemaker J. F. Cherry, A. Friedland. Neutrino Portal Dark Matter: From Dwarf Galaxies to IceCube. 2014. 20

[55] I. Yavin E. Izaguirre. New window to millicharged particles at the LHC. Phys. Rev., D92(3):035014, 2015. 20, 22

[56] S. Andreas et al. Proposal for an Experiment to Search for Light Dark Matter at the SPS. 2013. 21, 39

[57] V. Kozhuharov M. Raggi. Results and perspectives in dark photon physics. Riv. Nuovo Cim., 38(10):449-505, 2015. 21, 22, 23, 33, 38

[58] A. Ritz B. Batell, M. Pospelov. Exploring Portals to a Hidden Sector Through Fixed Targets. Phys. Rev., D80:095024, 2009. 22

[59] M. Pospelov. Secluded U(1) below the weak scale. Phys. Rev., D80:095002, 2009. 23

[60] D. Hanneke, S. Fogwell, G. Gabrielse. New Measurement of the Electron Magnetic Moment and the Fine Structure Constant. Physical Review Letters, 100(12):120801, 2008. 23

[61] R. Bouchendira, P. Clade, S. Guellati-Khelifa, F. Nez, F. Biraben. State of the art in the determination of the fine structure constant: test of Quantum Electrodynamics and determination of $h / m_{u}$. Annalen Phys., 525(7):484-492, 2013. 24 
[62] H. Davoudiasl, H. Lee, W. J. Marciano. Muon g-2 rare kaon decays, and parity violation from dark bosons. Phys. Rev., D89(9):095006, 2014. 25

[63] S. Boyarinov, V. Burkert, A. Deur, H. Egiyan, L. Elouadrhiri, et al. . HPS HEAVY PHOTON SEARCH. A Proposal to Search for Massive Photons at Jefferson LaboratorHPS Heavy Photon Search. December 2010. 25, 26, 38, $50,53,86,114,134$

[64] K. M. Zurek D. E. Morrissey, D. Poland. Abelian hidden sectors at a gev. Journal of High Energy Physics, 2009(07):050, 2009. 26

[65] N. Arkani-Hamed, D. P. Finkbeiner, T. R. Slatyer, N. Weiner. A Theory of Dark Matter. Phys. Rev., D79:015014, 2009. 26

[66] Ph. Schuster N. Toro J. D. Bjorken, R. Essig. New Fixed-Target Experiments to Search for Dark Gauge Forces. Phys. Rev., D80:075018, 2009. 27, 28

[67] A. Konaka et al. Search for Neutral Particles in Electron Beam Dump Experiment. Phys. Rev. Lett., 57:659, 1986. 32

[68] E. M. Riordan, M. W. Krasny, K. Lang, P. de Barbaro, A. Bodek, et al. . Search for short-lived axions in an electron-beam-dump experiment. Phys. Rev. Lett., 59:755-758, 1987. 32

[69] W. R. Nelson A. Abashian C. Church B. Lu L. W. Mo T. A. Nunamaker P. Rassmann J. D. Bjorken, S. Ecklund. Search for neutral metastable penetrating particles produced in the slac beam dump. Phys. Rev. D, 38:3375-3386, 1988. 32

[70] H. Nguyen Ngoc M. Davier. An Unambiguous Search for a Light Higgs Boson. Phys. Lett., B229:150-155, 1989. 32

[71] S. Pordes J. Volk S. Errede J. Wrbanek A. Bross, M. Crisler. Search for short-lived particles produced in an electron beam dump. Phys. Rev. Lett., 67:2942-2945, 1991. 32

[72] A. Ringwald S. Andreas, C. Niebuhr. New limits on hidden photons from past electron beam dumps. Phys. Rev. D, 86:095019, 2012. 33

[73] F. Bergsma et al. A Search for Decays of Heavy Neutrinos in the Mass Range 0.5-GeV to 2.8-GeV. Phys. Lett., 166B:473-478, 1986. 32

[74] S.N. Gninenko. Constraints on sub-gev hidden sector gauge bosons from a search for heavy neutrino decays. Physics Letters B, 713(3):244 - 248, 2012. 33

[75] J. Chauveau F. Dicarlo M. Dris et al. G. Bernardi, G. Carugno. Search for neutrino decay. Physics Letters B, 166(4):479 - 483, 1986. 33

[76] J. Blumlein and J. Brunner. New exclusion limits for dark gauge forces from beam-dump data. Physics Letters B, 701(2):155 - 159, 2011. 33, 34

[77] E. Perez del Rio. Dark Forces Searches at KLOE-2. Acta Phys. Polon., B47:461-470, 2016. 35 
[78] et al. J. P. Lees. Search for a Dark Photon in $e^{+} e^{-}$Collisions at BaBar. Phys. Rev. Lett., 113(20):201801, 2014. 35

[79] J.P. Lees et al. Search for invisible decays of a dark photon produced in e+ecollisions at BaBar. 2017. 35, 39

[80] et al. M. Ablikim. Dark Photon Search in the Mass Range Between 1.5 and $3.4 \mathrm{GeV} / \mathrm{c} 2.2017 .36$

[81] J. R. Batley et al. Search for the dark photon in $\pi^{0}$ decays. Phys. Lett., B746:178-185, 2015. 36, 39

[82] H. Merkel et al. Search at the Mainz Microtron for Light Massive Gauge Bosons Relevant for the Muon g-2 Anomaly. Phys. Rev. Lett., 112(22):221802, 2014. 37

[83] G. Agakishiev et al. Searching a dark photon with hades. Physics Letters B, 731:265 - 271, 2014. 38

[84] S. Abrahamyan et al. Search for a New Gauge Boson in Electron-Nucleus Fixed-Target Scattering by the APEX Experiment. Phys. Rev. Lett., 107:191804, 2011. 40

[85] M. Battaglieri et al. The Heavy Photon Search Test Detector. Nucl. Instrum. Meth., A777:91-101, 2015. 38, 45

[86] A. Celentano. The Heavy Photon Search experiment at Jefferson Laboratory. J. Phys. Conf. Ser., 556(1):012064, 2014. 41, 53

[87] R. Essig Z. Surujon B. Batell. Strong Constraints on Sub-GeV Dark Sectors from SLAC Beam Dump E137. Phys. Rev. Lett., 113(17):171802, 2014. 39

[88] I. Rachek B. Wojtsekhowski, D. Nikolenko. Searching for a new force at VEPP3. 2012. 39

[89] G. Ruggiero. The NA62 experiment at CERN. Nucl. Phys. Proc. Suppl., 210-211:216-219, 2011. 39

[90] et al. D. Banerjee. Search for invisible decays of sub-GeV dark photons in missing-energy events at the CERN SPS. Phys. Rev. Lett., 118(1):011802, 2017. 39, 42

[91] A. V. Artamonov, B. Bassalleck et al. Study of the decay K+ ,pi+nu,nu in the momentum region $<140.39$

[92] M. Perelstein D. Rubin D. J. Alexander, Y. Kubota and B. Wojtsekhowski P. Wittich. Cornell Dark Photon Search. In APS Meeting Abstracts, 2015. 39

[93] V. Kozhuharov M. Raggi and P. Valente. The PADME experiment at LNF. EPJ Web Conf., 96:01025, 2015. 39

[94] M. Anelli et al. A facility to Search for Hidden Particles (SHiP) at the CERN SPS. 2015. 39 
[95] G. Inguglia. Belle II studies of missing energy decays and searches for dark photon production. PoS, DIS2016:263, 2016. 40

[96] J. Balewski et al. The DarkLight Experiment: A Precision Search for New Physics at Low Energies. 2014. 40

[97] Y. Zhong B. Echenard, R. Essig. Projections for Dark Photon Searches at Mu3e. JHEP, 01:113, 2015. 40

[98] J. P. Lees et al. . Search for low-mass dark-sector higgs bosons. Phys. Rev. Lett., 108:211801, 2012. 40

[99] A. De Santis. The KLOE-2 Experiment at DAÎ̌̃NE. J. Phys. Conf. Ser., 770(1):012010, 2016. 40

[100] I. Jaegle. Search for the dark photon and the dark Higgs boson at Belle. Phys. Rev. Lett., 114(21):211801, 2015. 41

[101] G. Ovanesyan T. Peng M. J. Ramsey-Musolf C. A. ArgÃAEelles, X.-G. He. Dark Gauge Bosons: LHC Signatures of Non-Abelian Kinetic Mixing. Phys. Lett., B770:101-107, 2017. 41

[102] G. Aad et al. Search for long-lived neutral particles decaying into lepton jets in proton-proton collisions at $8 \mathrm{TeV}$ with the ATLAS detector. JHEP, 11:088, 2014. 43

[103] V. Khachatryan et al. A search for pair production of new light bosons decaying into muons. Phys. Lett., B752:146-168, 2016. 41, 44

[104] Ch. W. Leemann, D. R. Douglas, G. A. Krafft. The continuous electron beam accelerator facility: CEBAF at the Jefferson Laboratory. Annual Review of Nuclear and Particle Science, 2001. Vol. 51:413-450 (Volume publication date December 2001) https://doi.org/10.1146/annurev.nucl.51.101701.132327 . 46

[105] B. A. Mecking et al. The cebaf large acceptance spectrometer (clas). Nuclear Instruments and Methods in Physics Research Section A: Accelerators, Spectrometers, Detectors and Associated Equipment, 503(3):513 - 553, 2003. 46

[106] M. Spata H. Wang R. Kazimi, J. Hansknecht. Source and Extraction for Simultaneous Four-hall Beam Delivery System at CEBAF. page WEPFI085, 2013. 46

[107] N. Baltzell et al. The Heavy Photon Search beamline and its performance. Nucl. Instrum. Meth., A859:69-75, 2017. 49, 53

[108] C. Field. HPS Target. HPS Notes, September 2015. 50, 51

[109] P. Hansson, J. Jaros, T. Maruyama, O. Moreno,T. Nelson, M. Oriunno et al. .53

[110] S. Agostinelli et al. GEANT4: A Simulation toolkit. Nucl. Instrum. Meth., A506:250-303, 2003. 53, 64, 85 
[111] I. Balossino, et al. The HPS electromagnetic calorimeter. Nucl. Instrum. Meth., A854:89-99, 2017. 54, 55, 60, 62, 73, 74, 78, 79

[112] P. Hansson Adrian, C. Field, N. Graf, M. Graham et al. Heavy Photon Search Experiment at Je erson Laboratory:proposal for 2014-2015 run. May 2013. 59, 60, 134

[113] P. H. Adrian. The Silicon Vertex Tracker for the Heavy Photon Search Experiment. In Proceedings, 2015 IEEE Nuclear Science Symposium and Medical Imaging Conference (NSS/MIC 2015): San Diego, California, United States, page $7581862,2016.59$

[114] N. Graf, J. McCormick. Simulator for the linear collider (SLIC): A tool for ILC detector simulations. AIP Conf. Proc., 867:503-512, 2006. [,503(2006)]. 64

[115] R. Chytracek, J. McCormick, W. Pokorski, G. Santin. Geometry Description Markup Language for Physics Simulation and Analysis Applications. 53:2892 - 2896, 2006. 64

[116] J. Alwall, P. Demin, S. de Visscher et al. MadGraph/MadEvent v4: The New Web Generation. JHEP, 09:028, 2007. 64, 85

[117] F. Maltoni O. Mattelaer T. Stelzer J. Alwall, M. Herquet. Madgraph 5: going beyond. Journal of High Energy Physics, 2011(6):128, 2011. 64, 85, 87

[118] LCIO Home Page. 64

[119] N. Baltzell. ECal Pulse Fitting. HPS Notes, September 2015. 69

[120] N. Baltzell, R. Paremuzyan. Cluster-Track Matching. HPS Notes, September 2016. 71

[121] H. Szumila-Vance. HPS Ecal Timing Calibration for the Spring 2015 Engineering Run. HPS Notes, November 2015. 81

[122] A. F. Bielajew S. J. Wilderman W. R.Nelson H. Hirayama, Y. Namito. The EGS5 code system. 2005. 85

[123] M. Vanderhaeghen T. Beranek, H. Merkel. Theoretical framework to analyze searches for hidden light gauge bosons in electron scattering fixed target experiments. Phys. Rev., D88:015032, 2013. 85, 87, 88, 89, 92, 96

[124] T. Beranek. Theoretical analysis of hidden photon searches in high-precision experiments. Univ., Mainz, 2014. 87, 88

[125] M. Vanderhaeghen T. Beranek. Study of the discovery potential for hidden photon emission at future electron scattering fixed target experiments. Phys. Rev., D89(5):055006, 2014. 87, 88, 89, 90, 92, 140, 149, 150, 152

[126] T. Skwarnicki. A study of the radiative CASCADE transitions between the Upsilon-Prime and Upsilon resonances. PhD thesis, Cracow, INP, 1986. Appendix E. 106, 107 
[127] O. Moreno. Search for a Heavy Photon in the 2015 Engineering Run Data of the Heavy Photon Search Experiment. PhD thesis, UC Santa Cruz, 2016. https://www.jlab.org/indico/event/214/contribution/33/material/slides/0.pdf . 108, 115, 125, 134

[128] T. Kramer. Track parameters in LCIO. 2006. 115

[129] G. Cowan, K. Cranmer, E. Gross, O. Vitells. Asymptotic formulae for likelihood-based tests of new physics. Eur. Phys. J., C71:1554, 2011. [Erratum: Eur. Phys. J.C73,2501(2013)]. 124, 128, 129, 130, 131, 133

[130] A.D. Bukin. Fitting function for asymmetric peaks. ArXiv e-prints, November 2007. 124

[131] D. P. Kirkby W. Verkerke. The RooFit toolkit for data modeling. eConf, C0303241:MOLT007, 2003. [,186(2003)]. 124

[132] L. Lyons. Statistical Issues in Searches for New Physics. 2014. 127

[133] A. Roodman. Blind analysis in particle physics. eConf, C030908:TUIT001, 2003. [,166(2003)]. 127

[134] S. S. Wilks. The Large-Sample Distribution of the Likelihood Ratio for Testing Composite Hypotheses. Annals Math. Statist., 9(1):60-62, 1938. 129

[135] A. Wald. Tests of Statistical Hypotheses Concerning Several Parameters When the Number of Observations is Large. Transactions of the American Mathematical Society, 54(3):426-482, 1943. 130

[136] D. Coe. Fisher Matrices and Confidence Ellipses: A Quick-Start Guide and Software. 2009. 133 



\section{Acknowledgments}

"Let us be grateful to the people who make us happy; they are the charming gardeners who make our souls blossom."

\section{Marcel Proust}

In the end I want to take a moment and thank various people for their direct and indirect contribution to this work without whom this thesis would not be possible. First and foremost I offer my sincerest gratitude to my supervisors; Michel Guidal and Raphael Dupre for their support throughout my thesis, for their motivation, enthusiasm and patience whilst allowing me the room to work in my own way. Your guidance helped me through the research and the writing of this thesis. Raphael, in addition I am grateful for your invaluable help with administrative paperwork, for the many visits to dreadful prefecture office, and for many discussions about French history and culture.

A huge thank you goes to the Elias Khan, Achim Denig, Eric Armengaud and Marco Battaglieri for agreeing to be in my defense committee and reading my thesis so carefully. Special thanks should be given to Dr. Stepan Stepanyan, my research project supervisor for many years before my $\mathrm{PhD}$ and one of the referees of this thesis. His professional guidance, immense knowledge and valuable support have helped me to overcome the difficulties and gain practical knowledge.

I also want to thank you, my friends in Armenia, France, the USA and in other countries. I have always felt your love and support present throughout these $\mathrm{PhD}$ years, thank you for that. Hripsi jan, we spent my first New Year in France and that was one of the most fun holidays. During your short visits, we had a wonderful time discovering Paris and arguing about gris lapin. Narine, Armine and Vard, I will never forget our incredible adventures in Europe, which eventually lead me to im ktors. I am grateful to you for your friendship, as Armine would say, dzer mard tesaky shat em gnahatum. Let us do not forget here Hripsi again for introducing us all to each other. Mel jan, we met only a year ago, but you have become a close friend to me; it is as though I have known you for ages. Thank you for your hospitality during the 2016 New Year and the awesome birthday and for so many more things I can't list here. Yeranik jan, my all time roommate and Newport News co-survivor, we have been through so much together, I have been missing your travel-partnership and our long discussions about nothing and everything at the same time. My driving 
teachers, Arshak and above all great friends, you both supported me in one of my most stressful times in JLab. We have had great trips in Virginia as well as out of Virginia. I have great memories of our Armenian gang sipping wine at the beach in the evening or gathered at our place and tangling some topic, watching movie or making paper planes to see whose plane has the best aerodynamics. Rafo and Marianna, I am happy for you two and your cute small family. It always felt home being with you. Grigor jan, thank you for great many evenings in Yerevan, going to tango or to a rock concert or just for a cup of coffee. You were the important part of the midday gatherings at our office, I have missed you here. And of course Nerses and Hovik, I admire your knowledge and your patience. Thank you for all your help with many technical and software problems, and for all you have taught me.

Special thanks also to my friends at JLab: Kolya and Natasha, Andrey and Olya, Vanya Bedlinsky. Thank you for your company and your hospitality, for the many great evenings under your roof and for the delicious beach-barbeques.

Also I want to thank my colleagues and friends at IPNO for their company and support. Mohammed, my first office-mate and close friend in France, thank you for your friendship and many great adventures in the city. Wang Ying, no-one understood better than you the difficulties I went through during the PhD. Thank you for your support and for bringing a little bit of Chinese culture to our office. I hope you are happy with your new position. Dominique, thank you for interesting conversations and all your help and support, especially with organization of the reception after the defense. Olga and Gabriel, I am grateful for hosting me in the beginning, before I found a place to live. Silvia and your wonderful family, I had great time around the dinner table with you and I appreciated a lot your help in debunking the letters from the French bureaucratic "machine".

I want to specially thank the person who has been providing the coffee machine with supplies. It was probably the greatest help to my everyday work, so thank you kind man/woman.

No words are enough to express my gratitude to my family: my parents, who inspired me to think, study and work to pursue my goals and dreams; my sweetest sister, who makes the world a lighter and happier place for me; and her family with cutest and happiest little thing in the world, my nephew, with the power to blow all clouds away and make the sun to shine in the middle of a rainy, cloudy Paris November day.

To Wouter, the love of my life and my soulmate, you have supported me in every possible way during the last and hardest period of my PhD. I am incredibly lucky to have met you and can't say enough "thank you"s to the chain of all events and people that put me on the right world-line that led to you. Thank you for always being there for me in the bright and dark ages of the analysis and thesis production. I have a message for you that I received through inter-portal communication pigeons, I hope you know how to decode Fig. A.0.1. 


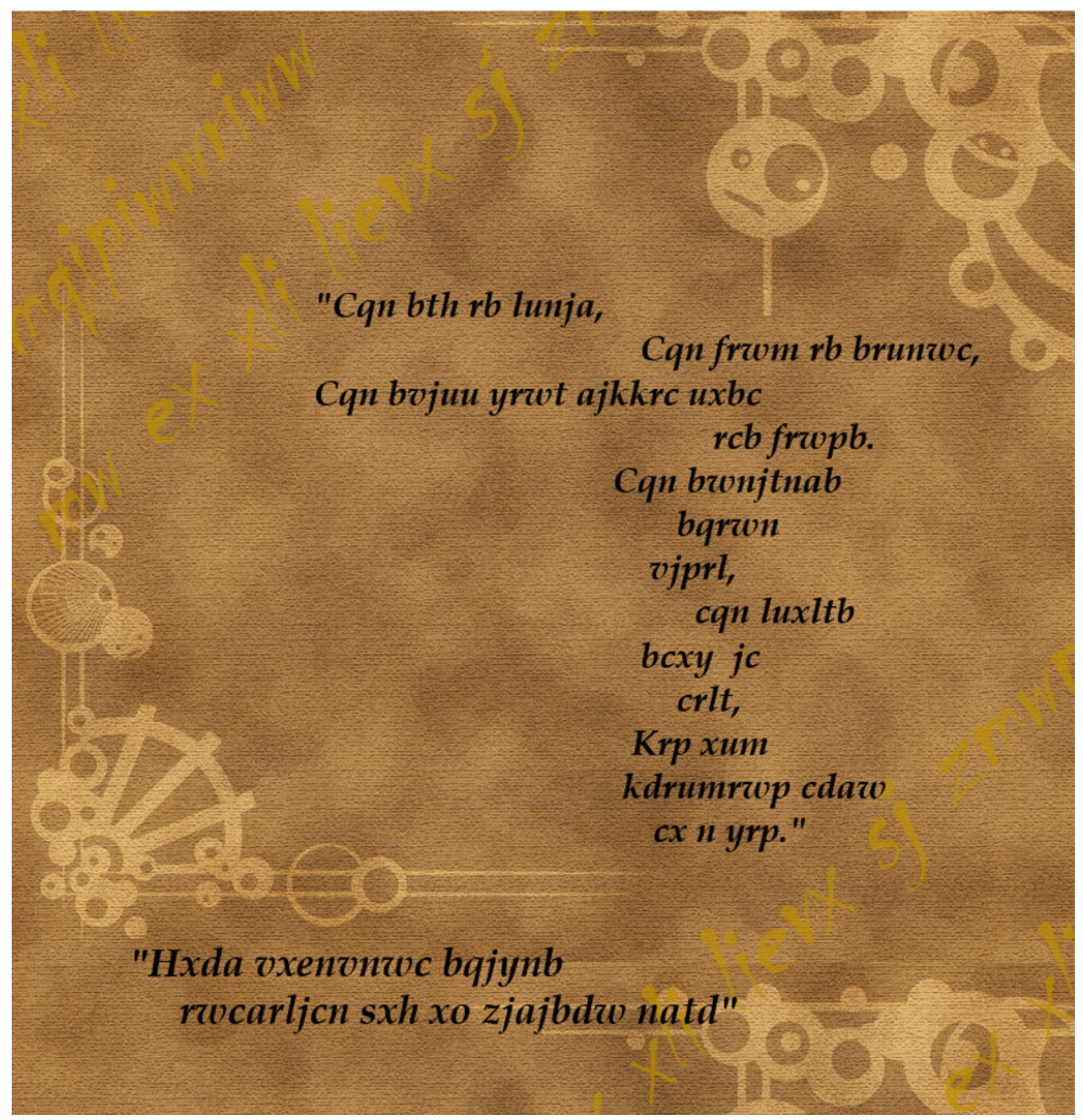

Figure A.0.1.: 
"Yet trees are not 'trees', until so named and seen and never were so named, till those had been who speech's involuted breath unfurled, faint echo and dim picture of the world."

J.R.R. Tolkien 



\section{universite $\dot{e}^{\bullet}$ \\ PARIS-SACLAY}

Titre : Recherche de photons sombres avec l'experience HPS au JLab

Mots clés : photon lourd, photon sombre, paraphoton, HPS, Recherche de photons sombres

Résumé : L'expérience HPS (Heavy Photon Search) au Jefferson Lab (USA) recherche un nouveau boson de jauge vecteur, nommé "photon lourd" ou "photon sombre", dans une fourchette de masse allant de 20 à $1000 \mathrm{MeV}$. Une telle particule couplerait avec le photon du modèle standard par effet de "kinetic mixing" et pourrait ainsi être émis par l'intermédiaire d'électrons. En utilisant un faisceau d'électron de haute intensité de un à six $\mathrm{GeV}$ envoyé sur une cible de tungstène, HPS cherche à détecter une fine résonnance dans le bruit de fond produit par les processus QED qui serait la signature d'un photon lourd. HPS exploitera aussi le fait qu'à très petit couplages, le photon lourd se désintègrera après une distance détectable, fournissant ainsi une seconde signature sous la forme d'un vertex éloigné de la cible.
Dans cette thèse, je présente les motivations pour une telle recherche de photon lourd dans ce domaine spécifique de l'espace de phase, puis je présente le spéctromètre HPS, en détaillant en particulier le calorimètre électromagnétique qui a été l'un de mes sujets d'étude. Ensuite, je présente mon travail utilisant une intégration Monte-Carlo pour calculer la section efficace des processus QED attendus dans l'expérience HPS. Finalement, je présente dans cette thèse mon analyse de donnée pour la recherche d'un pic sur le bruit de fond QED dans les données aquisent au printemps 2015 .

Title : Dark Photon Search with the HPS Experiment at JLab

Keywords : heavy photon, dark photon, dark sector photon, HPS, paraphoton, Heavy Photon Search

Abstract : The heavy photon search (HPS) experiment in Jefferson Lab (USA) is looking for a new vector gauge boson, called "heavy photon" or "dark photon", in a mass range between $20 \mathrm{MeV}$ and $1000 \mathrm{MeV}$. Such particle can couple to the Standard Model photon through kinetic mixing and therefore can be radiated in electron scattering. Using a highintensity, one to six $\mathrm{GeV}$ electron beam sent onto a tungsten target, HPS will look for a narrow resonance in the $e^{+} e^{-}$invariant mass spectrum above the QED background, that would be a signature of a dark photon. HPS will also exploit the fact that for small couplings, this dark photon would also travel a detectable distance before decaying, providing a second signature in the form of a vertex away from the target.
In this thesis, I will present the motivations to look for such a dark photon in this particular domain of phase space, then present the HPS spectrometer, with a particular highlight on the electromagnetic calorimeter which was a focus of my work. Then, I will present the studies that I carried out using a Monte-Carlo integration to calculate the cross section of the expected background QED processes for the HPS experiment. The final part of my work is focused on the data analysis, looking for a bump on the QED background, that I carried out using data taken in Spring 2015. 